\title{
THESIS ENTITLED
}

\section{"Beam Dynamics Studies and the Design, Fabrication and Testing of Superconducting Radiofrequency cavity for High intensity Proton Accelerator"}

\author{
SUBMITTED TO
}

DEPARTMENT OF PHYSICS AND ASTROPHYSICS

UNIVERSITY OF DELHI, DELHI - 110007

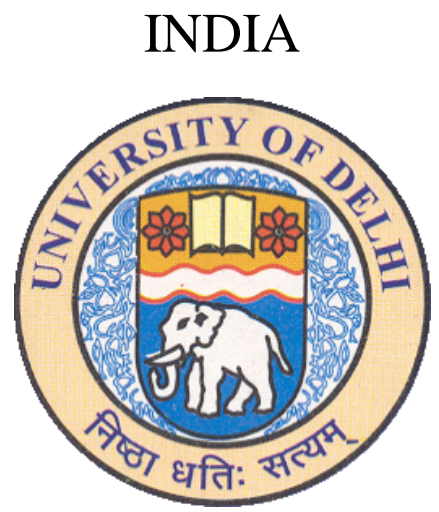

FOR THE DEGREE OF DOCTOR OF PHILOSOPHY

BY

\section{ARUN SAINI}

UNDER THE SUPERVISION OF

Dr. Kirti Ranjan

March, 2012 


\section{DECLARATION}

This thesis deals with the design, fabrication and testing of superconducting radio frequency cavities as well as beam dynamics studies of high intensity $\mathrm{H}^{-}$linear accelerator for the proposed Project-X facility at Fermilab. Most of the work presented in this thesis has been published or in the process of publication.

This work has been done under the supervision of Dr. Kirti Ranjan. This work has been done by the candidate himself and to the best of his knowledge; no part of this work has earlier been submitted for any degree or diploma of this or any other university.

(Dr. Kirti Ranjan)

Supervisor
(Arun Saini)

Candidate

(Prof. R. P. Tandon)

Head of the Department

Department of Physics and Astrophysics

University of Delhi

INDIA 


\section{Dedicated to...}

My loving parents, brother and sisters 


\section{Acknowledgments}

It was a long journey in search of Ph.D. During this four and a half years, I met many people who leave great impression on my professional as well as personal life. Their constant support, continuous encouragement and valuable advices allowed me to get where I am today. I would like to take this place to acknowledge these people.

Even my largest gratitude will be insufficient for my supervisor, Dr. Kirti Ranjan. His constant support, continuous encouragement and guidance throughout allowed me to enjoy this journey as much as destination. Even with his tight schedules with M.Sc. teaching classes and administrative work, he is actively involved in the work of all his students and always ready to discuss physics and to provide his prompt feedback. He is the best advisor and teacher I could have wished for. I have learned various things, from him, such as the way of thinking, and the way of proceeding in research, and so on. I had lot of discussions with him on various subjects other than physics such as art of living, religion etc. which helped me in the struggle for my own understanding. It was both a privilege and honour to work with him. His passion toward research and his work ethics will remain source of motivation for rest of my life. I would also like to thank Prof. Raghuvir Singh and Dr. Samit Mandal for being the part of my advisory committee and suggesting me Dr. Kirti Ranjan's name for my Ph.D. supervisor.

I would like to express my largest gratitude to head of the CDRST laboratory, Prof. R. K. Shivpuri, who interviewed me during my Ph.D admission and showed his faith on me. I benefited from his wide experience and useful comments at key moments. I also express my gratitude to him for providing outstanding working conditions at the laboratory and for his efforts and patience when reading my thesis report.

I wish to express my reverence and sense of deep gratitude to Dr. Ashutosh Bhardwaj for his invaluable, insightful comments and suggestions to improve the quality of 
this work. I am also obliged to the Head of the Department of Physics, Prof. R. P. Tandon for providing the necessary facilities in the Department.

As a student, I had the chance to work at Fermilab, U.S.A. I was the lucky beneficiary of the highly professional and organized people working there, and I would like to express sincere collective thanks to them, and in particular to Dr. Shekhar Mishra, Dr. Nikolay Solayk, Dr. Vyacheslav Yakovlev (Slava) and Dr. Jean Francoise Ostiguy.

Working within the RF and Linac beam dynamics group has been a real pleasure, thanks to the professional and human skills of the people belonging to it. I feel privileged to have been able to work within such a group of people, and I am giving warm thanks to all the group members: N. Solayk, Slava, J.P. Carneiro, J.F. Ostiguy, A. Lunin, I. Gonin, A. Vostrikov, N. Perunov and Timmer. Of course, I would like to thank the Fermilab director, Dr. P. Oddone and APC director Dr. V. Shiltsev to have supported my stay at the Fermilab.

I would like to thank many of them on a more personal level. I would like to express my sincere gratitude to Dr. Nikolay Solyak who accepted to become my mentor at Fermilab and invested so much of his precious time. Since day one, he was always there to help me out on every level and to support my work. His broad knowledge, highly diversified skills, experience, organization, patience, and kindness are all outstanding, and to have been under his supervision was a true privilege. I would also like to thank Slava who has been a great source of motivation. He shared many great stories from his work and his life experiences, which always enlightened my days. His amazing ability to come with simple solutions for any type of complicated problems, open mind, vast experience, and sense of humor have been constant source of refreshment, inspiration, and energy. I also express him my gratitude for helping me with editing of my thesis and articles for scientific conferences and peer reviews. I shared very enjoyable time with Slava. I owe lot of thanks to Jean Francoise Ostiguy who was always ready to give up some of his time to help me on numerous computer related problems and for constructive discussion.

I would also like to express my collective thank to Avinash Puntambekar, Manish Bagre, Jishnu Dwivedi, Rajeshwar Sandha, Prakash, S.C. Joshi, K.K. Pant, Shankar Lal, Arvind, Bhaskar Biswas, S.B. Roy for their invaluable time and discussions during my visit at RRCAT and IUAC. 
In India and U.S.A, I was surrounded by knowledgeable and friendly people who helped me daily. They helped creating an informal and congenial atmosphere that, in my opinion, makes research a much less onerous task than it otherwise might be. First and foremost, I gladly acknowledge my debt to my friend Ruchika Nayyar. Without her constant friendship, encouragement and advice, I would never have reached here. I can never forget our long discussions and also the wonderful time she has provided me as a friend and colleague during our visits to various research institutes and in particular to Fermilab, Banaras and Indore. Thanks are also due to Shilpi Jain, Sandhya Jain, Sudha Ahauja and Pooja Saxena, with whom I spent fruitful time. My junior colleagues Arun Kumar and Ajay Kumar deserve a special mention as they helped me a lot during my thesis submission.

There are many individuals who helped me during my stay at Fermilab. My collective thank to Shilpee Arora, Dilip Bhogadi, Suneel Dutt, Ashish Kumar, Mohammad Nayeem, Margie Bruce, Karen Swanson and Elaine Sivak.

In addition to the people in University and Laboratory, I am lucky enough to have the support of many good friends. Life would not have been the same without them. There are too many people to mention individually, but some names stand out. I wish to thank my friends in high school (Deepak Chauhan, Naveen Balayan and Youdhvir Singh), my friends as an undergraduate (Pankaj Sharma, Preeti Yadav, Pawan Khatri, Suman, Teena Bharal, Sharmeela), and my friend as a graduate student (Manish Verma), for helping me get through the difficult times, and for all the emotional support, camaraderie, entertainment, and caring they provided.

I also appreciate the continuous assistance I received from Mr. Mohammad Yunus and all CDRST lab staff members. It would, of course, be completely amiss for me to end my acknowledgments without recognizing the immense contribution that my family has made to my work. Their love and support has been a major stabilizing force over these past years. Their unquestioning faith in me and my abilities has helped to make all this possible and for that, and everything else, I dedicate this thesis to them. 


\section{Abstract}

The application horizon of particle accelerators has been widening significantly in recent decades. Where large accelerators have traditionally been the tools of the trade for high-energy nuclear and particle physics, applications in the last decade have grown to include large-scale accelerators like synchrotron light sources and spallation neutron sources. Applications like generation of rare isotopes, transmutation of nuclear reactor waste, sub-critical nuclear power, generation of neutrino beams etc. are next area of investigation for accelerator scientific community all over the world. Such applications require high beam power in the range of few mega-watts (MW). One such high intensity proton beam facility is proposed at Fermilab, Batavia, US, named as Project-X. Project-X facility is based on $\mathrm{H}^{-}$linear accelerator (linac), which will operate in continuous wave $(\mathrm{CW})$ mode and accelerate $\mathrm{H}^{-}$ion beam with average current of $1 \mathrm{~mA}$ from kinetic energy of $2.5 \mathrm{MeV}$ to $3 \mathrm{GeV}$ to deliver $3 \mathrm{MW}$ beam power. One of the most challenging tasks of the Project-X facility is to have a robust design of the $\mathrm{CW}$ linac which can provide high quality beam to several experiments simultaneously. Hence a careful design of linac is important to achieve this objective.

$\mathrm{H}^{-}$ion is non-relativistic at kinetic energy of $2.5 \mathrm{MeV}$ and its velocity changes very rapidly with acceleration in Project-X linac. Thus, the linac uses several types of accelerating structures which are optimized for different particle velocities to provide efficient acceleration. Project-X linac has evolved over recent years from pulsed version to continuous wave $(\mathrm{CW})$ version, so cavities are designed for both of these versions using simulation approach. In the pulsed (earlier) version of the linac, there already exists a 11-cell, $1.3 \mathrm{GHz}$ design of cavity. HOMs study of the cavity is performed and asymmetrical design of the end cell is proposed. Further, an alternate version of the cavity design based on 9-cell is also proposed. In latest $(\mathrm{CW})$ version of the linac, 
5-cell, $650 \mathrm{MHz}$ cavities are designed for the intermediate and high energy sections corresponding to $\beta_{G}=0.61$ and $\beta_{G}=0.90$. Shapes of these cavities are optimized to achieve maximum acceleration and minimum power dissipation to reduce cryogenic losses. A systematic study is performed to understand the effects of excitation of resonance of HOMs on beam quality and resultant power dissipation. Analysis of HOMs provides better understanding of the requirements of HOM damper for these cavities.

Further, a robust lattice is designed for reliable operation of CW linac which preserves beam quality and allows robustness in design parameters such as accelerating gradients in cavities, focusing gradient in magnets etc. Studies based on beam tracking codes are performed for the baseline lattice to analyze beam trajectory and beam emittance. An essential measure of a successful accelerator system is its ability to provide high beam availability and high reliability. To improve the reliability of linac, possibility of failure of beam line elements are included in the lattice design. Operation of the linac at CW mode puts stringent tolerances on beam transport elements, especially at low energy section, which increases the possibility of temporary or permanent loss of accelerating cavities and focusing magnets during the operation of linac. Lattice should be robust enough to have a capability that RF cavity or magnet failure may be compensated locally by using the neighbouring elements. The numbers of cavities and focusing magnets in a cryomodule are chosen in such a way that at least one failure of RF cavity or focusing magnet can be compensated locally.

A standard procedure developed for the fabrication of $1.3 \mathrm{GHz}$, 9-cell cavity at mass scale, is discussed in this work. Similar approach will be used for the fabrication of $650 \mathrm{MHz}$ cavities for the $\mathrm{CW}$ linac. Deep-drawing method is commonly used to fabricate Niobium cavity. Quality of inner surface of cavity degrades during process of fabrication which may limit maximum accelerating gradient in cavity. Thus, it is passed through sequence of surface treatments in order to remove defects from production process. Surface treatments involve chemical etching high pressure water rinsing and heat treatment of cavity. After all treatments, performance of a single-cell cavity like accelerating gradient and quality factor is tested at $2 \mathrm{~K}$ in the vertical-test stand (VTS) facility at Fermilab. 


\section{List Of Publication}

- "Superconducting RF cavity design study for the squeezed ILC section of the high intensity $\mathrm{H}^{-}$linac for the Project-X facility", Arun Saini, K. Ranjan, A. Lunin, S. C. Mishra, N. Perunov, N. Solyak, V. P. Yakovlev, Supercond. Sci. Technol. 25025024 (2012).

\section{Published Proceedings in International and National Conferences}

1. "Calculation of Acceptance of High Intensity superconducting proton linac for Project-X", A. Saini, C.S. Mishra, K. Ranjan, N. Solyak and V.P. Yakovlev, Particle Accelerator Conference (PAC), 2011, New York, USA.

2. "Study of effects of failure of Beam line elements \& their compensation in CW superconducting linac", A. Saini, C.S. Mishra, K. Ranjan, N. Solyak and V.P. Yakovlev, PAC 2011, New York, USA.

3. "Concept of EM designs of the $650 \mathrm{MHz}$ Cavities for the Project X", V.P. Yakovlev, A. Saini, M.S. Champion, I.G. Gonin, T.N. Khabiboulline, A. Lunin and N. Solyak, PAC 2011, New York, USA.

4. "Resonance effects of longitudinal HOMs in the Project X Linac", V.P. Yakovlev, A.Saini, I.G. Gonin, T.N. Khabiboulline, A. Lunin, N. Solyak, A.I. Sukhanov, and A. Vostrikov, PAC 2011, New York, USA.

5. "Physics design of the Project- X CW linac", N. Solyak, A. Saini, J.-P. Carneiro, J. Kerby, V.A. Lebedev, S. Nagaitsev, J.F. Ostiguy, A. Vostrikov and V.P. Yakovlev, PAC 2011, New York, USA. 
6. "Lattice design for superconducting CW linac for Project-X facility at Fermilab", A. Saini, K. Ranjan, J.P. Carneiro, S. Mishra, J.F. Ostiguy, N. Solyak, A.Vostrikov and V. Yakovlev Indian Particle Accelerator Conference (InPAC), 2011, New Delhi, India.

7. "Compensation of failure of RF cavity in CW superconducting linac", A. Saini, K. Ranjan, Solyak, S. Mishra and V. Yakovlev, InPAC 2011, New Delhi, India.

8. "RF cavities for superconducting CW linac for Project-X facility at Fermilab", A. Saini, K. Ranjan, S. Barbanotti, M.Champion, M.Foley, I.Gonin, C.Grimm, S. Kazakov, S. Mishra, L. Ristori, N. Solyak, A.Vostrikov and V.Yakovlev, InPAC 2011, New Delhi, India.

9. "Study of higher order modes in Project-X linac", A. Saini, K. Ranjan, I. Gonin, T.Khabilouline, S.Mishra, N.Solyak, A.Sukhanov, A.Vostrikov and V.Yakovlev, InPAC 2011, New Delhi, India.

10. "Design of superconducting cavities for $650 \mathrm{MHz}$ section of CW linac for ProjectX facility", A. Saini, K. Ranjan, S. Barbanotti, M.Champion, M.Foley, I.Gonin, C.Grimm, S. Kazakov, S. Mishra, L. Ristori, N. Solyak, A.Vostrikov and V.Yakovlev, InPAC 2011, New Delhi, India.

11. "650 MHz Option for High-energy Part of the Project X linac", V.P. Yakovlev, A. Saini, M.S. Champion, I.G. Gonin, S. Nagaitsev and N. Solyak, International Particle Accelerator Conference (IPAC) 2010 Kyoto, Japan.

12. "Longitudinal and Transverse Effects of HOMs in the Project X Linac", V. P. Yakovlev, A. Saini, T.N. Khabiboulline, N. Solyak and A. Vostrikov, IPAC 2010 Kyoto, Japan.

13. "Optimization of end cells of low beta cavity of higher energy part of Project X”, A. Saini, C.S. Mishra, K. Ranjan, N. Solyak and V.P. Yakovlev, IPAC 2010 Kyoto, Japan.

14. "Designing of 9 cell reduced beta elliptical cavity for high intensity proton linac", A. Saini, A. Lunin, C.S. Mishra, K. Ranjan, N. Solyak and V.P. Yakovlev, IPAC 2010 Kyoto, Japan. 
15. "Study of Coupler's Effects on ILC Like Lattice", A. Saini, A. Latina, A. Lunin, K. Ranjan, N. Solyak and V.P. Yakovlev, IPAC 2010 Kyoto, Japan.

16. "Implementation of coupler's effect in Lucretia", A. Saini, A. Latina, J.-F. Ostiguy, K. Ranjan and N. Solyak, PAC 2009, Vancouver, Canada. 


\section{Contents}

Acknowledgments i

Abstract

List Of Publication vii

List of Figures

List of Tables

1 Motivation and Framework: Proposed Project-X facility at Fermilab 1

1.1 Historical development of accelerators . . . . . . . . . . . 2 2

1.2 Requirement of high intensity proton or $\mathrm{H}^{-}$ion beam . . . . . . . 6 6

1.3 Choice of linear acceleration for high intensity beam . . . . . . . . . 10

1.4 High intensity beam facility at Fermilab: Project-X . . . . . . . . 10

1.4.1 Physics goals of Project-X . . . . . . . . . . . . . 11

1.4.2 Accelerator goals of Project-X . . . . . . . . . . . 14

1.5 Motivation for present work . . . . . . . . . . . . . . 17

1.6 Thesis Organization $\ldots \ldots \ldots \ldots \ldots$

2 Fundamentals of radio frequency cavity \& beam dynamics 23

2.1 Electromagnetic description of RF cavity . . . . . . . . . . . . . 24

2.1 .1 Maxwell's equations . . . . . . . . . . . . 24 24

2.1 .2 Cylindrical waveguide . . . . . . . . . . . . 25 25

2.1 .3 Cylindrical cavity . . . . . . . . . . . . . . . . 26

2.2 Multi-cell RF cavities . . . . . . . . . . . . . . . . . 28 28

2.3 Performance parameters of a cavity . . . . . . . . . . 30 
2.3 .1 Stored energy . . . . . . . . . . . . . . . 30

2.3 .2 Peak surface fields . . . . . . . . . . . . . . . 30

2.3 .3 Accelerating gradient . . . . . . . . . . . . . 31

2.3 .4 Transit time factor . . . . . . . . . . . . . . 31

2.3 .5 Quality factor ..................... 31

2.3 .6 Shunt impedance . . . . . . . . . . . . . . 33

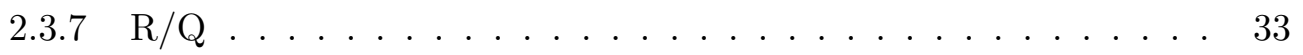

2.3 .8 Geometrical factor . . . . . . . . . . . . . . 34

2.4 Why superconducting (SC) cavity ? . . . . . . . . . . 34

2.5 Fundamentals of superconductivity . . . . . . . . . . 36

2.5.1 Type I and Type II superconductors . . . . . . . . . . . . . 37

2.5.2 Characteristics lengths and distinction between Type I and Type II superconductor . . . . . . . . . . . . . . . . . . . 38

2.5.3 Superconductivity in microwave fields . . . . . . . . . . 40

2.6 Choice of SCRF cavity material . . . . . . . . . . . . . . . 41

2.7 Limiting mechanisms in high gradient SCRF cavity . . . . . . . . . 42

2.7 .1 Multipacting . . . . . . . . . . . . . . . 43

$2.7 .2 \quad$ Field emission . . . . . . . . . . . . . . . . . . . 44

2.7.3 Thermal instability . . . . . . . . . . . . . . 45

2.8 Simulation tools for cavity design . . . . . . . . . . . . . . 46

2.8 .1 Numerical analysis . . . . . . . . . . . . . . . . . 47

$2.8 .2 \quad$ Boundary conditions . . . . . . . . . . . . . . . 4 47

2.8.3 Description of SLANS . . . . . . . . . . . . . . . 49

2.8.4 Incorporating practical aspects in simulation . . . . . . . . 49

2.9 Beam dynamics . . . . . . . . . . . . . . . . . . . 50

2.9.1 Coordinate system . . . . . . . . . . . . . . 50

2.9 .2 Longitudinal synchrotron motion . . . . . . . . . . . . 51

2.9.3 Transverse betatron motion . . . . . . . . . . . . . . 54

2.9.3.1 RF defocusing by accelerating structure . . . . . . . 58

2.10 High intensity effects in linear accelerator . . . . . . . . . . . . 59

2.10 .1 Space charge effect . . . . . . . . . . . . . . . 559

2.10 .2 Image charge effect . . . . . . . . . . . . . . . . 6 62

2.11 Beam dynamics codes for lattice design . . . . . . . . . . . . 6 62 
3 Design of SCRF cavity for the Project-X linac 65

3.1 Design of cavity geometry . . . . . . . . . . . . 66 66

3.1 .1 Fundamental aspects . . . . . . . . . . . . 67 67

3.1 .2 Practical aspects . . . . . . . . . . . . . 71

3.1 .3 Design of inner cell . . . . . . . . . . . . . 771

3.1.3.1 Influence of geometrical variables . . . . . . . . . 73

3.1 .4 End cell design . . . . . . . . . . . . . . 78

3.1 .5 HOMs damping requirement . . . . . . . . . . . 79 79

3.2 Design of SCRF cavity for the pulsed linac . . . . . . . . . . . . 8 80

3.2.1 HOMs study for symmetrical 11-cell SILC cavity . . . . . . . . . 82

3.2 .2 Design of 9-cell SILC cavity . . . . . . . . . . . . . 89

3.3 Design of SCRF cavity for the CW linac . . . . . . . . . . . 96

3.3.1 Why transition from $325 \mathrm{MHz}$ to $650 \mathrm{MHz}$ frequency instead of $1.3 \mathrm{GHz} ． \ldots \ldots \ldots \ldots \ldots \ldots$

3.3.2 Design of medium beta cavity $\left(\beta_{G}=0.61\right)$ for intermediate energy section of linac . . . . . . . . . . . . . 98

3.3.3 Design of high beta cavity $\left(\beta_{G}=0.90\right)$ for high energy section of $\operatorname{linac} \ldots \ldots \ldots \ldots \ldots \ldots \ldots$

3.3.4 Requirement of HOM damper for $650 \mathrm{MHz}$ cavities . . . . . . . . 111 3.3.4.1 Beam Time Structure . . . . . . . . . . . . . . . 112

3.3.4.2 Resonance excitation of HOMs . . . . . . . . . . . 113

3.3.4.3 Collective effects in CW linac . . . . . . . . . . . 121

4 Beam dynamics study of Project-X CW linac 123

4.1 Assumptions and principles of lattice design . . . . . . . . . . . 123

4.2 SC linac architecture of Project-X . . . . . . . . . . . . . 125

4.2 .1 Low energy section . . . . . . . . . . . . . . . . . 125

4.2.2 Intermediate and High energy sections . . . . . . . . . . . . 129

4.3 Beam optics for baseline design of the Project-X linac . . . . . . . 130

4.4 Acceptance of the linac . . . . . . . . . . . . . . . 133

4.5 Reliability of the linac . . . . . . . . . . . . . . . 137

4.5.1 Failure of RF elements . . . . . . . . . . . . . . . 138

4.5.2 Local compensation scheme . . . . . . . . . . . . . . 138 
4.5.3 Failure of RF cavity . . . . . . . . . . . . . . . . 140

4.5.3.1 Study and local compensation of failure of first cavity in SSR0 section . . . . . . . . . . . . . 140

4.5.3.2 Study and local compensation of failure of last cavity in SSR0 section . . . . . . . . . . . . . . . 143

4.5.3.3 Study and compensation of failure of last cavity in SSR2 section . . . . . . . . . . . . . . 146

4.5.4 Failure of magnetic element . . . . . . . . . . . . . . 147

4.5.4.1 Study and compensation of failure of first solenoid in SSR0 section . . . . . . . . . . . . . . . . . 148

4.5.4.2 Study and compensation of failure of first solenoid in SSR1 section . . . . . . . . . . . . . . . 149

5 Cavity fabrication and characterization 151

5.1 Cavity fabrication . . . . . . . . . . . . . . . . . . 151

5.1 .1 Niobium sheet . . . . . . . . . . . . . . . 152

5.1 .2 Forming half cells: Deep drawing method . . . . . . . . . . 153

5.1 .3 Electron beam welding $(\mathrm{EBW}) \ldots \ldots \ldots \ldots$

5.1 .4 Inspections and qualification tests: . . . . . . . . . . . 156

5.1.4.1 Vacuum leak test . . . . . . . . . . . . 157

5.1 .4 .2 Optical inspection . . . . . . . . . . . . 158

5.2 Cavity surface processing . . . . . . . . . . . . . . 160

5.3 Mechanical tuning of RF cavity . . . . . . . . . . . . . . . 165

5.4 Experimental measurement of cavity: Vertical test . . . . . . . . . 168

5.4.1 Measurement of Quality factor . . . . . . . . . . . . . 171

5.4 .2 Measurement of Electric field level . . . . . . . . . . . . . 172

5.4.3 Measurement of single cell ILC cavity: TE1CAT003 . . . . . . . 172

5.5 Cavity installation in cryomodule . . . . . . . . . . . . 175

6 Summary

$\begin{array}{ll}\text { References } & 181\end{array}$ 


\section{List of Figures}

1.1 Principle of Wideroe's RF linac . . . . . . . . . . . . . . 2

1.2 Principle and electric field profile of an Alvarez linac. . . . . . . . . . . 4

1.3 Proposed Project-X site . . . . . . . . . . . . . . . . . . . 11

1.4 Schematic layout of Kaons rare decay experiment. . . . . . . . . . . 12

1.5 Schematic layout of muon to electron conversion experiment . . . . . . . 13

1.6 Schematic layout of the proposed Project-X facility at Fermilab. . . . . 14

1.7 Acceleration scheme for $3 \mathrm{GeV} \mathrm{SC} \mathrm{CW} \mathrm{linac} \mathrm{.} \mathrm{.} \mathrm{.} \mathrm{.} \mathrm{.} \mathrm{.} \mathrm{.} \mathrm{.} \mathrm{.} \mathrm{.} \mathrm{.} \mathrm{.} \mathrm{.} 14$

1.8 Ion source and LEBT . . . . . . . . . . . . . . . . . 15

1.9 A proposal for beam distribution to different experiments at Project-X facility . . . . . . . . . . . . . . . . . 16

2.1 Single cell and multi-cell cavity with beam pipe. . . . . . . . . . . 28

$2.2 \pi$ mode field distribution in multi-cell cavity . . . . . . . . . . . 29 29

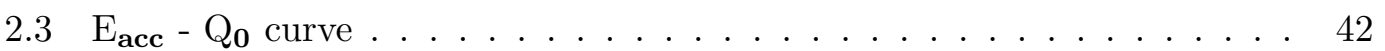

2.4 Exploiting symmetry in the geometry . . . . . . . . . . . . . . 48

2.5 Longitudinal phase stability principle . . . . . . . . . . . . 52

2.6 Longitudinal phase stability of an ion beam . . . . . . . . . . . 553

2.7 Courant-Snyder ellipse . . . . . . . . . . . . . . . . . . 56

2.8 Beam phase space in presence of non linear force . . . . . . . . . . 5 57

2.9 Field pattern in RF gap . . . . . . . . . . . . . . . . . . . . 58

3.1 Energy gain per cavity $\ldots \ldots \ldots \ldots$. . . . . . . . . . . 69

3.2 Operating range of cavity . . . . . . . . . . . . . . 69

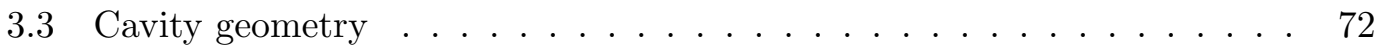

$3.4 \mathrm{R}_{\text {iris }}$ and coupling coefficient . . . . . . . . . . . 74 


\section{LIST OF FIGURES}

3.5 Variation in field enhancement factors and $\mathrm{R} / \mathrm{Q}$ with iris radius . . . . 74 74

3.6 Influence of semi axes A and B of equatorial elliptical arc . . . . . 76

3.7 Influence of semi axes $a$ and $b$ of iris elliptical arc . . . . . . . . 76

3.8 End cell design . . . . . . . . . . . . . . . . . . . . . . . . 78

3.9 Project-X pulse SC linac . . . . . . . . . . . . . . . . . 80 80

3.1011 -cell cavity . . . . . . . . . . . . . . . . . . 82

3.11 HOM spectrum for 11-cell cavity . . . . . . . . . . . . . 83

3.12 3rd monopole passband of symmetrical 11 cell cavity . . . . . . . . 84

3.13 Trapped modes in 11-cell cavity . . . . . . . . . . . . . . . . . 84

3.14 HFSS result for trapped mode . . . . . . . . . . . . . . . 85

3.15 HOM spectrum for asymmetrical 11-cell cavity . . . . . . . . . . 87

3.16 3rd monopole passband of asymmetric 11 cell cavity . . . . . . . . . 87

3.17 Most concerned modes in asymmetrical 11-cell cavity . . . . . . . . . . 88

3.18 Comparison of symmetrical and asymmetrical 11-cell cavity for (a) $\mathrm{Q}_{e x}$ and $(\mathrm{b})$ impedances $\left(\mathrm{R} / \mathrm{Q} * \mathrm{Q}_{e x}\right)$ of concerned monopole modes in third pass band. . . . . . . . . . . . . . . . . . . 8 89

3.19 Optimization curve for inner cell for $R_{\text {iris }}=30 \mathrm{~mm}$. . . . . . . . . 91

3.20 Optimization curve for inner cell for various values of $R_{\text {iris }}$. . . . . . . 91

3.21 A symmetric 9-cell SILC cavity geometry . . . . . . . . . . . 93

3.229 cell cavity . . . . . . . . . . . . . . . . . . . . . 93

3.23 HOMs spectrum for 9 cell-cavity . . . . . . . . . . . . . . 94

3.24 Beta dependence of the monopole HOM with high effective impedance. . 95

3.25 Electric field distribution for the most concerned monopole modes . . . 95

3.26 Comparison of acceleration efficiency of $1300 \mathrm{MHz}$ and $650 \mathrm{MHz}$ cavities. 97

3.27 Variation of field enhancement factors with $\beta \ldots \ldots$. . . . . . . . 98

3.28 5-cell $\beta_{G}=0.61$ cavity. . . . . . . . . . . . . . . . . 99

3.29 Cavity dimensions . . . . . . . . . . . . . . . . . . 100

3.30 Field distribution of fundamental mode in 5 -cell $\beta_{G}=0.61650 \mathrm{MHz}$ cavity.102

3.31 (a)Distribution of effective impedance R/Q $(\Omega$ ) of longitudinal modes (monopole modes) and (b) transverse effective impedance $(R / Q)_{\|}\left(\Omega / \mathrm{cm}^{2}\right)$ of transverse modes (dipoles) for $\beta_{G}=0.61 \ldots \ldots \ldots . \ldots 10 \ldots$

3.32 Distribution of monopole modes (longitudinal modes) with phase advance for first six pass bands of 5 -cell, $\beta=0.61,650 \mathrm{MHz}$ cavity. . . . . 104 
3.33 Distribution of dipole modes (transverse modes) with phase advance for first six pass band of 5 -cell, $\beta_{G}=0.61,650 \mathrm{MHz}$ cavity. . . . . . . . 105

3.345 -cell $\beta_{G}=0.90$ cavity. . . . . . . . . . . . . . . . . . 106

3.35 Surface fields distribution in $\beta_{G}=0.90$ cavity . . . . . . . . . . . 107

3.36 (a)Distribution of effective impedance R/Q $(\Omega)$ of longitudinal modes (monopole modes) and (b) transverse effective impedance $(R / Q)_{\|}\left(\Omega / \mathrm{cm}^{2}\right)$ of transverse modes (dipoles) for $\beta_{G}=0.90 \ldots \ldots \ldots$. . . . . 108

3.37 Variation in HOM impedances with $\beta$ in $\beta_{G}=0.90$ cavity . . . . . . . 108

3.38 Distribution of monopole modes (longitudinal modes) with phase advance for first six pass band of $\beta_{G}=0.90,650 \mathrm{MHz}, 5$-cell cavity. . . . . 109

3.39 Distribution of dipole modes (transverse modes) with phase advance for first six pass band of $\beta_{G}=0.90,650 \mathrm{MHz}, 5$-cell cavity. . . . . . . . 110

3.40 Variation in frequency bandwidth of fifth pass band with (a) $R_{\text {iris }}$ and

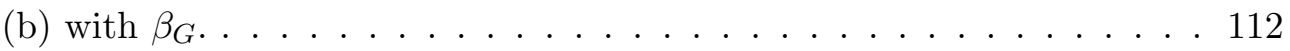

3.41 Beam separation scheme at the end of CW linac at Project-X facility. . 113

3.42 Beam spectrum for Project-X CW linac . . . . . . . . . . . . . . 114

3.43 (a)Power loss distribution for the $\beta_{G}=0.61$ cavity for $\mathrm{Q}=10^{10}$. $\beta=$ $0.61, \beta=0.54$ and $\beta=0.73$ are considered for calculation of power losses.(b) Power losses due to HOM excitation for $\beta=0.61$ cavity as a function of beam velocity $(\beta) \ldots \ldots \ldots \ldots \ldots \ldots$

3.44 Power losses in $\beta_{G}=0.90$ cavity $\ldots \ldots \ldots \ldots \ldots$

3.45 Power loss reduction by HOM frequency shift for a (a) $\beta_{G}=0.61$ and (b) $\beta_{G}=0.90$ cavity as a function of $\beta$ for $\mathrm{Q}=10^{10} \ldots \ldots \ldots \ldots \ldots$

3.46 Quality factor for monopole pass band of $\beta_{G}=0.90$ and $\beta_{G}=0.61$ cavity 117 $3.47 \delta f_{\epsilon}$ dependence on $\beta$ velocity for (a) $\beta_{G}=0.61$ and (b) $\beta_{G}=0.90$ cavity. 120 3.48 Probability of having significant emittance growth for (a) $\beta_{G}=0.61$ and (b) $\beta_{G}=0.90$ cavity. . . . . . . . . . . . . . . 120

4.1 Focusing period in low energy section $\ldots \ldots \ldots \ldots . \ldots \ldots$

$4.2 \quad \mathrm{SSR} 0$ cavity . . . . . . . . . . . . . . . . . 127

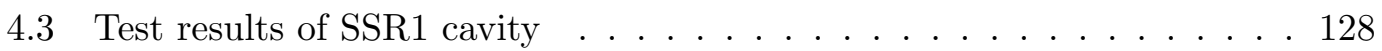

4.4 Focusing period in intermediate and high energy subsection . . . . . . 129

4.5 Zero current phase advance in linac . . . . . . . . . . . . . . . 131 


\section{LIST OF FIGURES}

4.6 Gradients and effective voltage in cavity . . . . . . . . . . . . . 131

4.7 Focusing magnet field in solenoids and quadrupoles . . . . . . . . . 132

4.8 Beam parameters for baseline design . . . . . . . . . . . . . 133

4.9 Acceptances of the baseline lattice . . . . . . . . . . . . . . . 134

4.10 Distribution of lost particles in the linac during acceptance calculation. 135

4.11 Longitudinal acceptance through transition between neighbouring section 136

4.12 Beam profile after failure of first cavity in SSR0 section . . . . . . . . . 141

4.13 Acceptances of linac after failure of first cavity in SSR0 section . . . . . 142

4.14 Local compensation scheme for failure of first cavity in SSR0 section . . 142

4.15 Beam profile after applying local compensation scheme for the failure of first cavity in SSR0 section . . . . . . . . . . . . . . 143

4.16 Beam quality parameters after applying local compensation scheme for the failure of first cavity in SSR0 section . . . . . . . . . . . . . . . . 144

4.17 Beam envelope after failure of last cavity in SSR0 . . . . . . . . . . . 145

4.18 Beam emittances comparison after applying local compensation scheme for the failure of last cavity in SSR0 section . . . . . . . . . . . . 146

4.19 Beam profile after the failure of first solenoid in SSR0 section . . . . . . 148

4.20 Beam emittance after the failure of first solenoid in SSR0 section . . . . 149

4.21 Beam emittance after the failure of first solenoid in SSR1 section . . . . 150

5.1 Cavity fabrication steps . . . . . . . . . . . . . . 152

5.2 Eddy current scanner setup at Fermilab . . . . . . . . . . . . . . 153

5.3 Scanned result of $\mathrm{Nb}$ sheet . . . . . . . . . . . . . . . . . . 154

5.4 Dies for half cell formation . . . . . . . . . . . . . . . . . 154

5.5 Deep drawing method for half cell forming . . . . . . . . . . . . 155

5.6 Forming tools and half cell cup . . . . . . . . . . . . . 155

5.7 Production of defocused electrom beam . . . . . . . . . . . . 157

5.8 Sketch of optical inspection system . . . . . . . . . . . . . 158

5.9 Overview of optical inspection system . . . . . . . . . . . 159

5.10 Optical inspection of TE1CAT003 cavity . . . . . . . . . . . 159

5.11 Effect of material removal on cavity gradient . . . . . . . . . . 160

5.12 Schematic of EP system . . . . . . . . . . . . . . . . . 162

5.13 EP system ANL . . . . . . . . . . . . . . . . . . . 162 
5.14 Schematic of HPWR . . . . . . . . . . . . . . . . 165

5.15 Tuning machine at Fermilab . . . . . . . . . . . . . . 166

5.16 Tuning mechanism for RF cavity. . . . . . . . . . . . . . . . . 166

5.17 Tuning of multicell cavity . . . . . . . . . . . . . . . 167

5.18 Cavity alignment before and after tuning. . . . . . . . . . . . 168

5.19 Schematic of vertical test stand . . . . . . . . . . . . . . 169

5.20 Schematic of experimental arrangement for vertical test. . . . . . . . . 170

5.21 Performance of single cell ILC type TE1CAT003 cavity. . . . . . . . . . 172

5.22 Defect in TE1CAT003 cavity. . . . . . . . . . . . . . . . 173

5.23 Another defect location in TE1CAT003 cavity. . . . . . . . . . . . . 174

5.24 Cavity surface after tumbling and light EP treatment. . . . . . . . . . 174

5.25 Performance of single cell ILC type TE1CAT003 cavity after surface treatment. . . . . . . . . . . . . . . . . . . . . 175 


\section{List of Tables}

2.1 List of Niobium properties . . . . . . . . . . . . . . . . 41

3.1 Geometry used to understand influence of geometrical parameters of cavity. 77

3.2 A summary of cavity design procedure . . . . . . . . . . . . 77

3.3 Basic parameters of $1.3 \mathrm{GHz}$ section of linac. . . . . . . . . . . . . 81

$3.41 .3 \mathrm{GHz} \beta_{G}=0.81$ 11-cell cavity parameters $\ldots \ldots \ldots$

3.5 Eigen mode frequency spectrum after end cell optimization. . . . . . . . 86

3.6 $\mathrm{Q}_{e x}$ and $\mathrm{R} / \mathrm{Q}$ for the concerned monopole modes in the third pass band. 88

3.7 $\mathrm{Q}_{e x}$ and $\mathrm{R} / \mathrm{Q}$ for the most concerned dipole modes in the first three pass bands of SILC cavities. . . . . . . . . . . . . . . . . . . . 90

3.8 Geometrical parameters for inner and end cell of 9-cell $\beta_{G}=0.811 .3$ $\mathrm{GHz}$ cavity. . . . . . . . . . . . . . . . . . 92

3.9 RF Parameters for fundamental mode of 9-cell $\beta_{G}=0.811 .3 \mathrm{GHz}$ cavity. 94

3.10 Geometrical parameters for half inner and half end cell of 5-cell, $\beta_{G}=$ $0.61,650 \mathrm{MHz}$ cavity. . . . . . . . . . . . . . . . 99

3.11 Geometrical parameter for half cell of 5 -cell, $\beta_{G}=0.61,650 \mathrm{MHz}$ cavity at different stages. . . . . . . . . . . . . . . . . . 101

3.12 RF Parameters for fundamental mode of 5 -cell $\beta_{G}=0.61,650 \mathrm{MHz}$ cavity.101

3.13 Geometrical parameter for half cell of 5-cell, $\beta_{G}=0.90,650 \mathrm{MHz}$ cavity at different stages. . . . . . . . . . . . . . . . . 106

3.14 RF Parameters for fundamental mode of 5-cell, $\beta_{G}=0.90,650 \mathrm{MHz}$ cavity.107

$4.1 \quad$ SSR0 cavity parameters. . . . . . . . . . . . . . . . . . 127

4.2 SSR1 cavity parameters. . . . . . . . . . . . . . . . . . 128

$4.3 \quad$ SSR2 cavity parameters. . . . . . . . . . . . . . . . 129 


\section{LIST OF TABLES}

4.4 Number of elements in each section along with transition energy. . . . . 130

4.5 Beam parameters for the baseline design of the linac. . . . . . . . . . 132

4.6 Beam parameters at the end of linac. . . . . . . . . . . . . 145

4.7 Beam parameters at the end of linac. . . . . . . . . . . . . 146

4.8 Beam parameters at the end of linac. . . . . . . . . . . . . . 147

4.9 Beam parameters at the end of linac. . . . . . . . . . . . . . 149

4.10 Beam parameters at the end of linac. . . . . . . . . . . . . 150

5.1 Vacuum leak test for TE1CAT003 cavity after EB welding. . . . . . . 157 


\section{Motivation and Framework: Proposed Project-X facility at Fermilab}

The application horizon of particle accelerators has been widening significantly in recent decades. Where large accelerators have traditionally been the tools of the trade for high-energy nuclear and particle physics, applications in the last decade have grown to include large-scale multi-purpose accelerators like synchrotron light sources and spallation neutron sources. Applications like generation of rare isotopes, transmutation of nuclear reactor waste, sub-critical nuclear power, tritium production, radiation damage studies, studies of rare processes and generation of neutrino beams etc. are other areas of interesting investigation for accelerator scientific community all over the world. Such applications require high beam power in the range of few mega-watts (MW). One such high intensity proton beam facility is proposed at the Fermi national accelerator laboratory (FNAL), Batavia, USA, named as Project-X [1, 2]. This chapter gives a brief overview of historical development of accelerators and potential applications of high intensity proton or $\mathrm{H}^{-}$ions accelerators. The main physics objectives of the Project-X facility and their global imprints on the scientific community are discussed. Layout of proposed accelerator complex for the Project-X facility is presented. Finally, the motivation behind the work and the thesis organization are described. 


\section{MOTIVATION AND FRAMEWORK: PROPOSED PROJECT-X FACILITY AT FERMILAB}

\subsection{Historical development of accelerators}

Initial experiments in nuclear physics using charged particle scattering with a target was performed by E. Rutherford [3] in 1911 which resulted in a growth of relatively new science, the quantum mechanics. This experiment provided an idea to use charged particle beams as main instrument in the investigation of nuclear structure. Rutherford used alpha particles from a natural radioactive isotope and thus, the beam parameters were uncontrolled practically. The quest of providing highly intense, controlled beam and increasing the kinetic energy of charged particle motivated the physicists to design machine, named as accelerator, which could accomplish these requirements. The simplest way to increase the energy is to allow the particle beam to move through a high electric potential. Energy gained by a particle depends on potential difference between the two electrodes. Therefore, the initial development of particle accelerators was related to the design of high voltage generators. Van de Graaff generator [4] and Cockroft-Walton generator [5] are good examples of such high voltage generators. These high voltage generators are called electrostatic accelerators because voltage between the electrodes remains constant with time. The performance of electrostatic accelerators are restricted by maximum achievable voltage between electrodes which is limited by a high voltage break down.

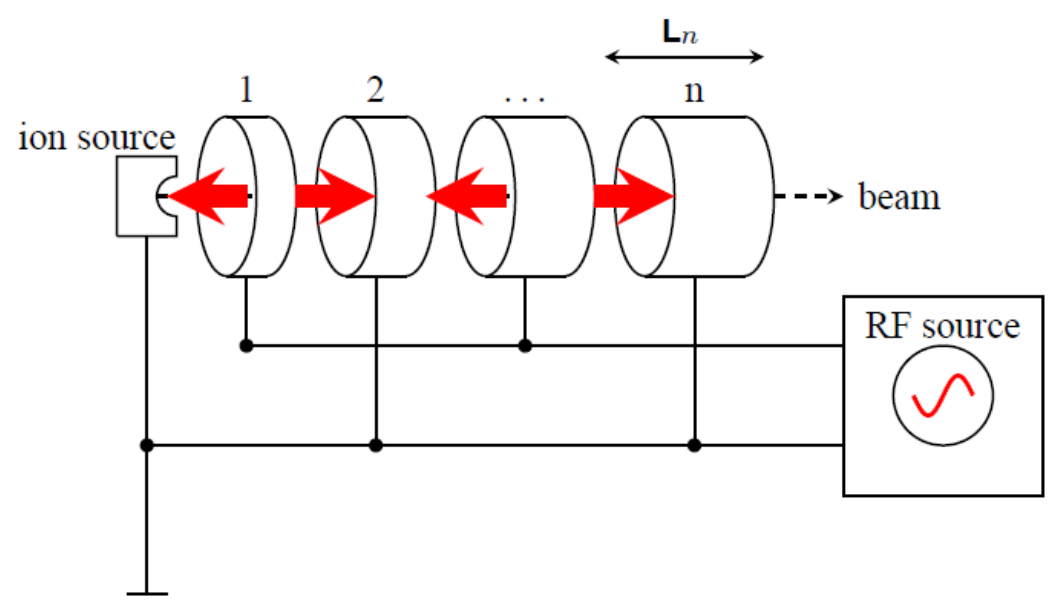

Figure 1.1: RF electric field (shown by arrow) between drift tubes accelerate the particles. 
In 1925 G. Ising [6] suggested an idea to use oscillating electric field to avoid the limitation of electrostatic accelerator and achieving further higher energy. The principle of using oscillating field for acceleration was first demonstrated by R. Wideroe [7] in 1927-1928. The basic idea of operation of the Wideroe accelerator is shown in Figure 1.1. A key component of Wideroe accelerator was metallic tubes which was aligned along the beam trajectory. Subsequent metallic tubes are connected to opposite polarities of a radio frequency $(\mathrm{RF})$ source. Wideroe accelerator operates at $\pi$-mode. It means that at any instant electric field configuration between two successive gaps differ in phase by $\pi$. Ions are accelerated in a gap between the metallic tubes. When the field becomes decelerating the ions drift inside the tube where no field is applied to them. Therefore, these metallic tubes are also called drift tubes. To perform continuous acceleration the distance between centers of two subsequent gaps $L_{n}$ should satisfy the condition:

$$
L_{n}=\frac{v}{2 f}
$$

where $f$ is the frequency of the RF source and $v$ is the velocity of particle. The particle velocity increases during acceleration and therefore, to maintain synchronization condition, distance between the gaps has to increase accordingly. Even though Wideroe established the principle that unlike an electrostatic accelerator, the voltage gain of the RF accelerator could exceed the maximum applied voltage but its performance was still limited for two reasons:

- For higher velocities the length of the drift tubes has to increase, which means that there was a natural practical limit for these machines. Especially for light charged particles the drift tubes would simply become too long.

- Operation at higher frequency results in reduction of drift tube length. But there is another limitation. Since these metallic tubes are not enclosed by a conducting boundary, the operation at higher frequencies means that the drift tubes were basically becoming antennas. With increasing frequency they radiate more and more of the RF energy instead of using it for acceleration, thus leading to a poor efficiency of the accelerator.

A solution was proposed by Louis Alvarez [8, who put the Wideroe linac into a volume enclosed by conducting wall. This solved the problem of radiated energy at 


\section{MOTIVATION AND FRAMEWORK: PROPOSED PROJECT-X FACILITY AT FERMILAB}

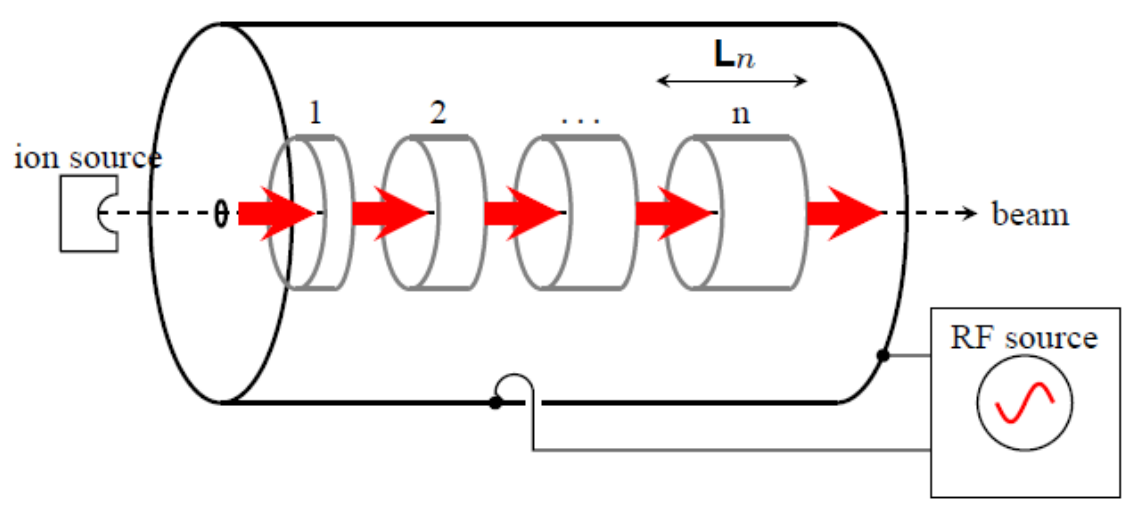

Figure 1.2: Principle and field profile (shown by arrow) of an Alvarez linac.

higher frequencies. Figure 1.2 shows the principle of the Alvarez linac. It can be noticed that in the Alvarez linac the field, in all gaps, points in the same direction. Synchronism with the RF demands that the RF phase changes by $2 \pi$ while the particles travel from one gap to the next. Thus, distance between centers of two subsequent gaps $L_{n}$ is:

$$
L_{n}=\frac{v}{f}
$$

Trajectory of a particle through Alvarez and Wideroe accelerators was linear so these are called linear accelerators (linac).

In 1929 E.O. Lawrence [9] invented circular accelerator where charged particle moves in spiral trajectory under the influence of uniform magnetic field. As particle moves in circular or spiral orbit, particle can pass through an accelerating gap many times. Over the period of times Lawrence circular accelerator has been developed into synchrotron for the acceleration of particles up to the energy in tera electron volt $(\mathrm{TeV})$. Large hadron collider (LHC) at CERN is a synchrotron accelerator which accelerates proton beam up to the kinetic energy of $3.5 \mathrm{TeV}$.

Initial development of accelerators was based on acceleration of heavy ions. For the acceleration of electron which becomes relativistic at relatively low energy, new accelerating structures were developed. A traveling wave could be used to accelerate relativistic particles. A cylindrical waveguide propagates waves with phase velocities greater than the speed of light. Since the charged particles must be traveling at less than the speed of light, they will not obtain any net acceleration, because they can not 
keep in phase with the wave. If the waveguide is loaded by putting some obstacles, the phase velocity of the wave can be slowed down to a usable value. The particles may then "surf" along the wave with a phase yielding an accelerating force.

A traveling wave structure, closed at both ends with metallic walls, will yield multiple reflections on the end walls until a standing wave pattern is established. This type of accelerating structures are called standing wave accelerating structures. While the longitudinally open traveling wave structure allows all frequencies, additional boundary condition in longitudinal direction for standing wave structure results in an existence of discrete frequencies. If one feeds RF power at a different frequency, the excited fields will be damped exponentially. Thus, these structures can be excited only for certain loss free electromagnetic modes. Standing wave structure is commonly known as resonant cavity or resonator and frequency of operation is called resonant frequency. Resonators are widely used for both ions and electron acceleration at all energies while traveling wave structures are used only for relativistic beam i.e., $\beta\left(=\frac{v}{c}\right) \sim 1$ with $c$ being the velocity of light.

In modern accelerators variety of accelerating structures are used to accelerate the beam for kinetic energy ranging from few $\mathrm{KeV}$ to several hundred $\mathrm{GeV}$. On the basis of the particle velocity accelerating structures are divided broadly in two groups.

- Low velocity accelerating structures : $\beta<0.5$.

- Medium and high velocity accelerating structures : $0.5 \leq \beta \leq 1.0$.

Drift tube linac (DTL) which is a modified Alvarez accelerating structure, cavity based on coaxial transmission wave guide such as half wave resonator (HWR), quarter wave resonator (QWR) and spoke resonator (SR) are commonly used as low velocity accelerating structures. Normal conducting DTL, coupled cavity drift tube linac (CCDTL) and coupled cavity linac (CCL) are used in linac for spallation neutron source (SNS) [10] facility at Oak Ridge while HWR are used at ATLAS facility. Rare isotope facility (RIA) consider HWR for the acceleration of beam at low energy [11.

Over the period of time cylindrical shaped cavity has been evolved into a elliptical shaped cavities for acceleration of intermediate and high velocity particles. These are significantly efficient and provide high accelerating gradient. Multi-gaps elliptical shaped superconducting cavity are used in modern accelerator to maximize net acceleration over the length of accelerator. 


\section{MOTIVATION AND FRAMEWORK: PROPOSED PROJECT-X FACILITY AT FERMILAB}

\subsection{Requirement of high intensity proton or $\mathrm{H}^{-}$ion beam}

Scientific perspectives of accelerator facilities based on high intensity proton or $\mathrm{H}^{-}$ion beam acceleration are discussed below.

- Spallation neutron source: The neutrons are produced when an ion beam of energy typically in the range of $1 \mathrm{GeV}$ to $3 \mathrm{GeV}$ hits a target of heavy nuclei (lead, bismuth). Excited nuclei decay into stable states by releasing neutrons, gamma rays and electrons.

Distinctive properties of neutrons make them ideal probes for investigations of condensed matter, which are summarized below [12]:

1. The absence of the charge and the small cross-section of interaction allow investigating bulk materials in the form of thick targets.

2. Scattering on nuclei allows hydrogen "imaging" and identifying isotopes.

3. Neutron magnetic moment allows us to examine magnetic parameters at the micro-level.

4. The wavelength of thermal neutrons at inter-atomic distances allows us to determine the crystalline structure and the arrangement of atoms in a lattice.

- Generation of neutrino beam: Our present understanding of elementary particle physics is based on the Standard Model (SM) of particle physics. Neutrinos are elementary particles which have no charge and virtually no mass according to the SM. In the past decade tremendous progress has been made toward the better understanding of neutrinos but there are still lot of open questions about their absolute masses, neutrino oscillations and possibilities of charge-parity violation. In recent years, serious evidences follow from the experiments on neutrino that were carried out in Gran Sasso (Italy) [13] and Kamioke (Japan) [14] about the existence of physics that does not fit the framework of the SM. The question whether neutrino has or has not mass became decisive for justification of principles of the theory of weak interactions, and, on the other hand, it is decisive for solving problems in Astrophysics. The experiments with electron neutrino $\nu_{e}$ from the Sun, and also with $\nu_{e}$ and $\nu_{\mu}$ from the space in the atmosphere showed 
that it is quite possible that neutrino oscillates between the $\nu_{e}, \nu_{\mu}$ and $\nu_{\tau}$ states. At least two of these neutrinos have masses different from zero.

Precise measurements of neutrino mass and evidences for new physics related with them require high flux neutrino beam. A high intensity proton (or $\mathrm{H}^{-}$) beam is bombarded on high atomic number $(Z)$ target which results in production of $\pi$ mesons as one of secondary particles. These $\pi$ mesons decay into muons when they pass through a long channel. Primary modes of $\pi$ meson decay are :

$$
\begin{aligned}
& \pi^{+} \rightarrow \mu^{+}+\nu_{\mu} \\
& \pi^{-} \rightarrow \mu^{-}+\overline{\nu_{\mu}} \\
& \pi^{0} \rightarrow 2 \gamma
\end{aligned}
$$

Thus, $\mu^{+}$and $\mu^{-}$are generated using these decay modes. As $\mu^{ \pm}$live 100 times longer than $\pi^{ \pm}$, a linear muon decay channel would need to be few tens of kilometer long. To avoid this difficulty, muons are injected into a storage ring with long straight section. Accumulated beams of $\mu^{+}$are the sources of electron neutrino $\left(\nu_{e}\right)$ and muon anti-neutrino $\left(\overline{\nu_{u}}\right)$ neutrino, and beams of $\mu^{-}$mesons generate electron anti-neutrino $\left(\overline{\nu_{e}}\right)$ and muon neutrino $\left(\nu_{\mu}\right)$. Decay modes of muons are given as follows:

$$
\begin{aligned}
& \mu^{+} \rightarrow e^{+}+\nu_{e}+\overline{\nu_{\mu}} \\
& \mu^{-} \rightarrow e^{-}+\overline{\nu_{e}}+\nu_{\mu}
\end{aligned}
$$

This approach to neutrino production in storage rings forms the basis for circular $\mu$-colliders where center of mass energy in the regime of $10 \mathrm{TeV}$ can be achieved.

- Transmutation of nuclear wastes: Power reactors do not extract all of the energy contained in their uranium (or uranium/plutonium) fuel. Spent fuel contains radioactive isotopes of heavy elements such as plutonium and americium and fission products. Some of these isotopes remain radioactive for many thousands of years. The disposal of nuclear wastes is a major concern for countries with active nuclear power program. Most countries are committed to a program of long-term geologic disposal, that is, stabilization of the radioactive material and burial in specially-designed receptacles and repositories. These plans continue to solicit the concern of some experts and many members of the public. As an alternative 


\section{MOTIVATION AND FRAMEWORK: PROPOSED PROJECT-X FACILITY AT FERMILAB}

to long-term storage, it has been proposed that the long-life isotopes be converted to shorter-lived ones through exposure to neutrons. The transmutation reaction can be induced by spallation neutrons. An accelerator-driven nuclear waste transmutation system would consist of three major sub-systems [15]:

- A high intensity proton accelerator with beam power of $5 \mathrm{MW}$ for a demonstration, up to $\sim 50 \mathrm{MW}$ for an industrial operation.

- A burner reactor where spallation and transmutation would occur.

- a processing plant in which short-lived isotopes that could not undergo further transmutation would be removed for secure disposal, and where other isotopes could be recycled into new fuel assemblies for the burner reactor.

- Production of radioactive ion beam (RIB): Rare and highly unstable nuclei can be produced by bombarding heavy nuclei with proton beam or intense flux of spallation neutrons. These exotic nuclei are excellent means of studying fundamental interaction between nucleons. The two production methods are used in RIB facilities. One is commonly called Isotope Separation On Line (ISOL) and the other is called In Flight. In ISOL type facilities, radioactive ions are produced essentially at rest in a thick target, that is bombarded with energetic primary particles from a driver accelerator. After diffusion out of the target and ionization the radioactive ions can be accelerated in a post-accelerator. For the in-flight method an energetic heavy ion beam is fragmented while passing through a thin target. After mass, charge and momentum selection in a fragment separator the selected ions can be analyzed or stored for further studies. No post-acceleration is required. While the ISOL method allows good quality low energy RIBs to be produced, in-flight facilities are optimum for higher energy RIBs of short-lived nuclei. In order to move closer to the extreme limits of stability the available driver beam intensities have to be increased.

- Accelerator driven sub critical reactor system (ADS): ADS is a relatively new concept which is still needed to be demonstrated. The principal advantages of ADS over the critical reactors are twofold: greater flexibility with respect to fuel composition, and potentially enhanced safety. ADS are ideally suited for burning fuels which are otherwise problematic for critical reactor operation. Burning 
fuels such as $U^{233}$ and minor actinide, degrade neutronic characteristics of the critical core to unacceptable levels due to small delayed neutron fractions and short neutron lifetimes. Additionally, ADS allows the use of non-fissile fuels (e.g. Th). ADS is based on spallation neutrons which are generated by high intensity proton accelerator under irradiation of the target made of a material with heavy nuclei. The deficiency of neutrons in the reactor operating in subcritical mode is compensated on the account of the spallation neutrons. The optimum energy of a proton beam compensating the deficiency of the neutron flux in the subcritical reactor is in the range of 1 to $3 \mathrm{GeV}$. ADS also provides enhanced safety due to the fact that once the accelerator is turned off, the system shuts down in the absence of spallation neutrons. Therefore, ADS seems to have the potential to provide an additional route to an efficient and economic nuclear power generation with the available uranium and thorium resources. The major sub-systems of ADS are [16]:

- High power proton accelerator: It is capable of accelerating the 10 $30 \mathrm{~mA}$ beam up to $1 \mathrm{GeV}$.

- Spallation target: Heavy elements such as lead, bismuth for 10 - $30 \mathrm{MW}$ beam power.

- Subcritical core: Fast neutron system, thermal neutron system or a combination of fast and thermal neutron system.

Worldwide interest is increasing in high-current $(>10 \mathrm{~mA})$, high-power $(>1 \mathrm{MW})$ proton accelerators. The main existing and proposed facilities based on high intensity linac all over the world are spallation neutron source (SNS) facility [10] at Oak Ridge National Laboratory (ORNL), Project-X facility at Fermi National Accelerator Laboratory (FNAL) [2], European Spallation Source (ESS) facility [17, European Isotope Separation On-Line Radioactive Nuclear Beam (EURISOL) facility [18], Japanese Hadron Project (JPARC) [19] and many more are still to come. SNS facility is already working successfully.

Most of the facilities are based on high intensity proton or $\mathrm{H}^{-}$ion beam linear accelerators. Thus, development of high intensity ion linear accelerators provides benefits in the framework of a multipurpose facility. 


\section{MOTIVATION AND FRAMEWORK: PROPOSED PROJECT-X FACILITY AT FERMILAB}

\subsection{Choice of linear acceleration for high intensity beam}

All the proposed or existing high intensity ion beam facilities use linac for first stage of acceleration. The choice is made due to its capabilities for producing high energy and high intensity charge particle beams with high beam quality. Some attractive features of the linac are following :

- High beam quality: Linac is a single-pass machine. The beam traverses its path only once. Thus, repetitive error conditions causing destructive beam resonances are avoided which are common feature in circular accelerators where beam passes through orbit multiple times.

- Capability of handling high current beam: In comparison with synchrotron, linac can achieve high beam quality and have more distributed beam losses. Thus, linac can operate at higher duty factor, even at 100\% (continuous wave mode), which results in accleration of beam with high average current.

- Easy injection and extraction: Since nominal trajectory of beam through linac is a straight line, there is no need for special techniques for efficient injection and extraction of the beam.

- Synchrotron radiation: When a charge particle traverses a circular trajectory, it looses its energy in the form of synchrotron radiation. Synchrotron radiation is negligible in linac relative to circular accelerators. Therefore, linacs are efficient for acceleration even for charged particles with relatively smaller masses.

- Reliability and maintainability: Linac can be classified as an array of modules consisting of accelerating and focusing elements which are arranged in a straight line. It is easy to maintain and to replace failed elements in linac relative to circular accelerator as one needs to replace only the affected module with new one. Future upgrade of accelerator to higher energies is easier for linac by adding more modules at the end of linac.

\subsection{High intensity beam facility at Fermilab: Project-X}

Fermilab is one of the largest laboratory in Unites States for fundamental research based on high energy particle physics. Fermilab's Tevatron was highest energy accelerator 
until LHC started operation in 2010. In recent times, focus of accelerator community

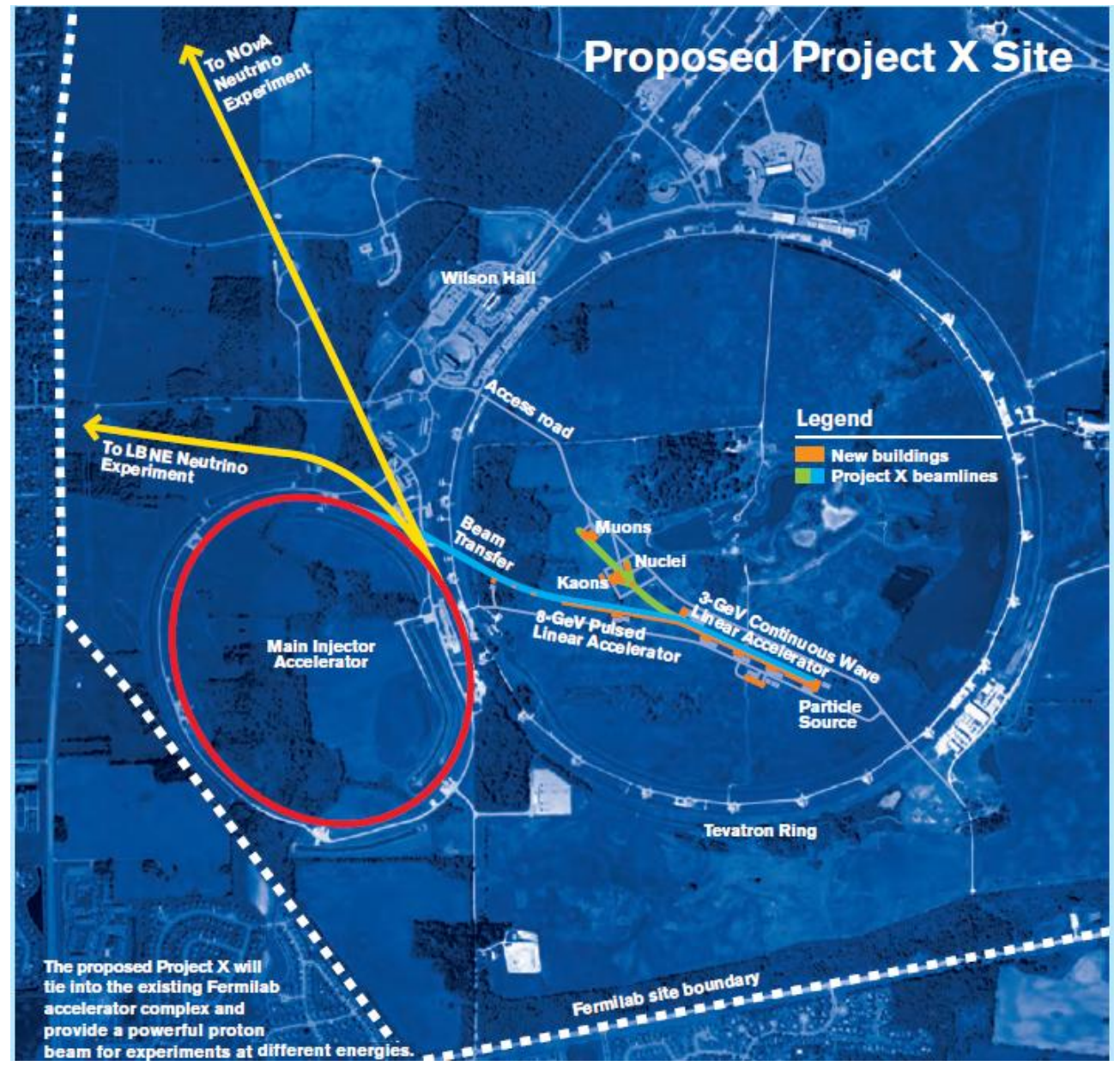

Figure 1.3: Proposed Project-X site at Fermilab.

is moving toward high intensity ion accelerators and Fermilab also decided to move toward high intensity frontier. A multi mega watt proton accelerator facility named as Project-X is proposed for construction at Fermilab [1, 20]. It is the centerpiece of the plan for future development of the Fermilab accelerator complex. Figure 1.3 shows proposed site for Project-X facility. It will be nicely tied with existing accelerator complex and will provide high intensity proton beam of different energies. Project-X will be a multiuser facility which supports many experiments simultaneously.

\subsubsection{Physics goals of Project-X}

The Main objectives of Project-X facility are following: 


\section{MOTIVATION AND FRAMEWORK: PROPOSED PROJECT-X FACILITY AT FERMILAB}

\section{- A neutrino beam for long baseline neutrino oscillation experiments:}

Most of the previous experiments on neutrinos were based on cosmic rays which resulted in excess of background and very low frequency of desired events. A high intensity neutrino source of a single flavour reduces backgrounds and its known energy spectrum and intensity could be decisive both for oscillation searches and precision measurement of the lepton mixing parameters.

- Kaons rare decay experiments: A neutral kaon decays into a neutral pion and two neutrinos, $K^{0} \rightarrow \pi^{0}+\nu+\bar{\nu}$. This process is a unique probe for study of the matter-antimatter asymmetry in our world and is a strong adjudicator of the existence of physics beyond the SM. The proposed Project-X accelerator would create enough beam power to pursue the kaon physics. Figure 1.4 shows schematic layout of proposed Kaon decay experiment. Project X facility, with a

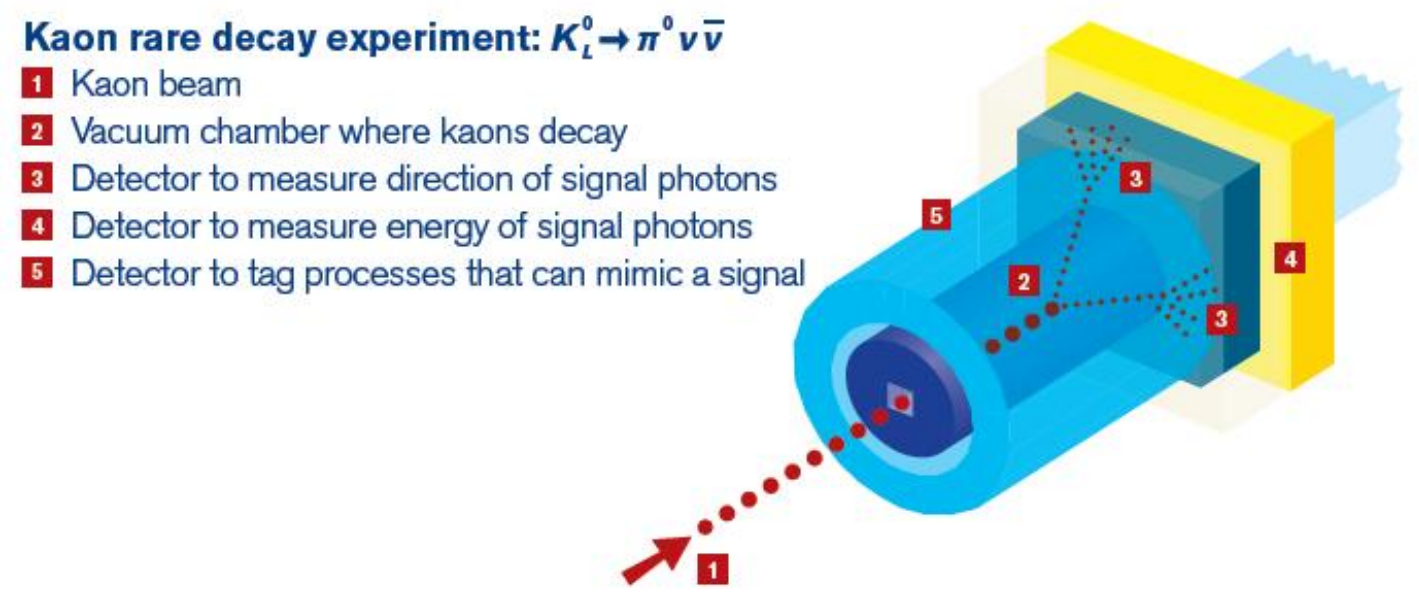

Figure 1.4: Schematic layout of Kaons rare decay experiment.

50-picosecond pulse fired every 40 nanoseconds, is ideally suited for time-of-flight techniques that are needed to determine with high accuracy the momentum of neutral kaons before they decay in the vacuum chamber. The design of the photon detector is optimized to precisely measure the energy and direction of the two photons emerging from the decay of the neutral pion and a detector surrounding 
the vacuum chamber will help identify background processes that can mimic a signal.

- Advanced muon to electron (mu2e) conversion experiment: Quarks transform into each other, and so do neutrinos. Scientists have never observed the charged leptons (electron, muon, and tau) change directly into each other, yet there exists a possibility that these processes may exist. Proposed experiment "Advanced muon to electron conversion" at Project-X facility will hunt for these processes. High intensity proton beam will produce huge number of muons

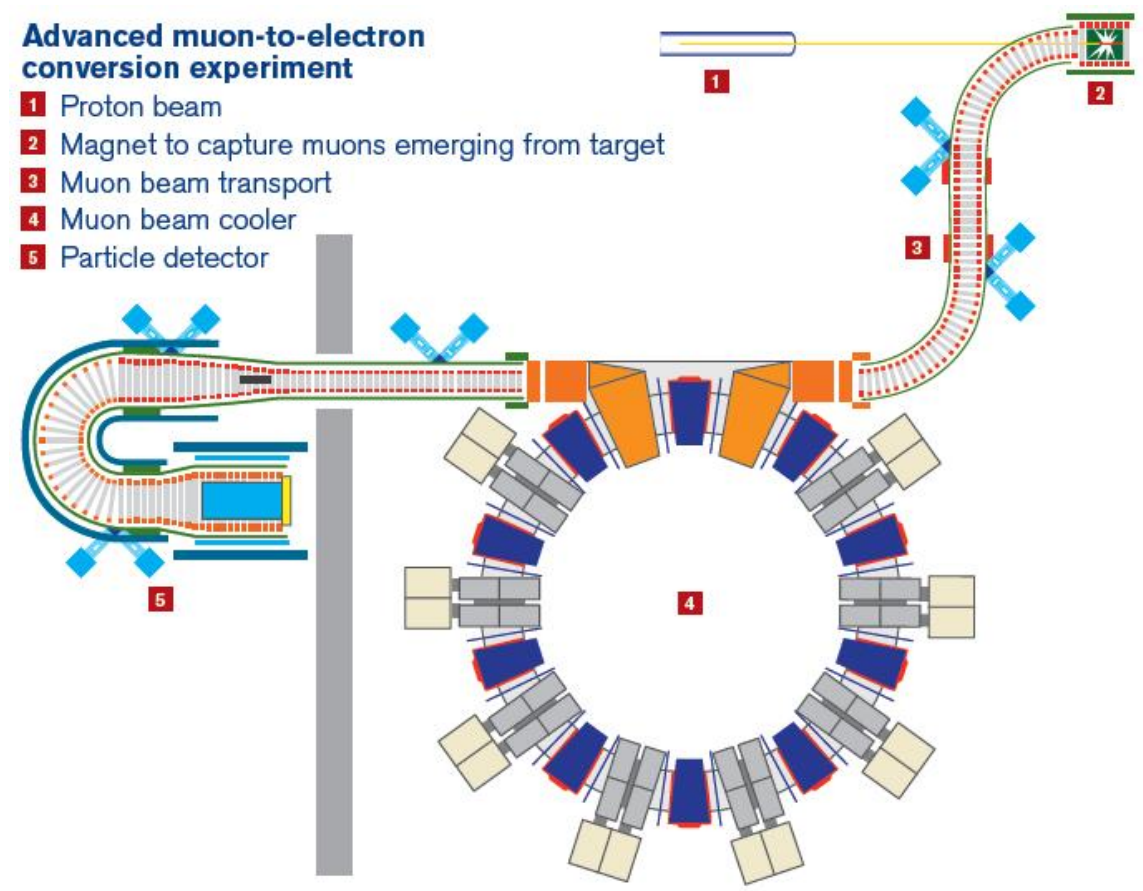

Figure 1.5: Schematic layout of muon to electron conversion experiment.

in controlled environment. These muons are captured and then state-of-the-art beam cooling techniques would reduce the momentum range of the muon beam by a factor of 10 compared to current experiments. Combined with a high-precision electron detector, this low momentum spread, high-flux muon beam would allow scientists to explore the mystery of charged-lepton conversion in greater depth than ever before. Figure 1.5 shows schematic layout of muon to electron conversion experiment. 


\section{MOTIVATION AND FRAMEWORK: PROPOSED PROJECT-X FACILITY AT FERMILAB}

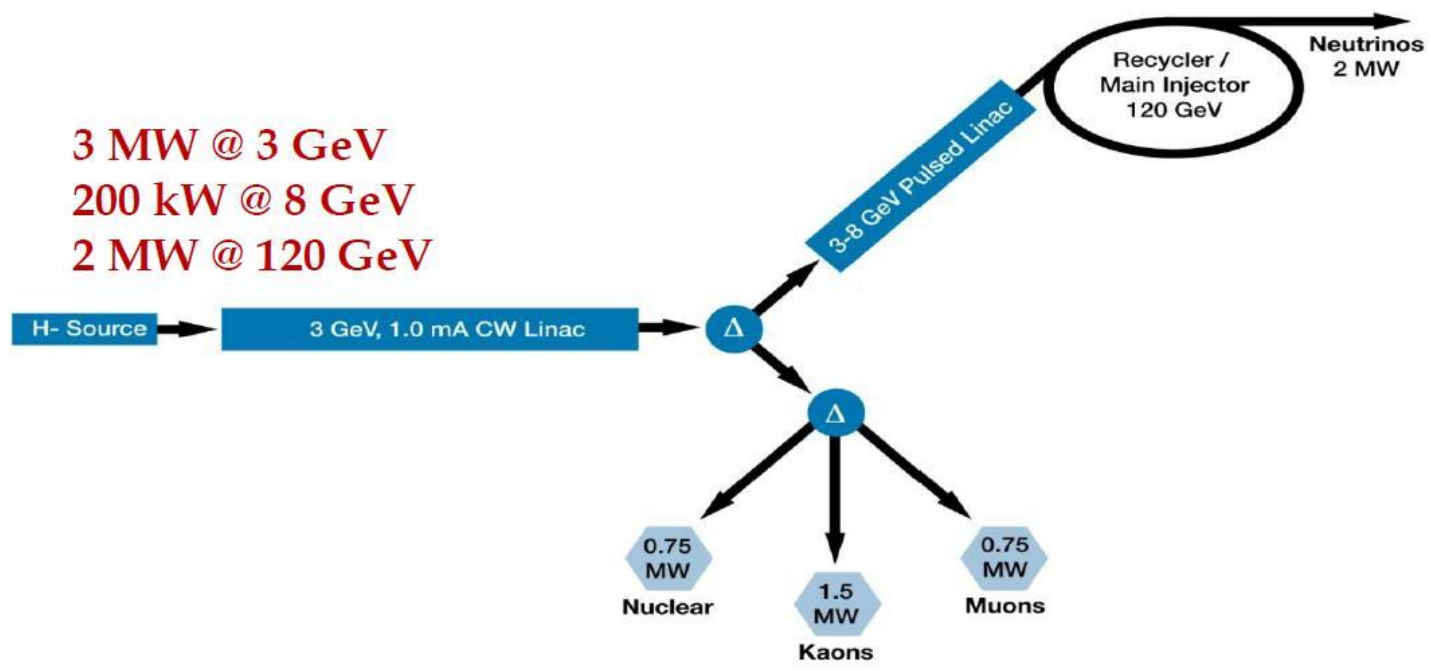

Figure 1.6: Schematic layout of the proposed Project-X facility.

\subsubsection{Accelerator goals of Project-X}

An overview of Project- $\mathrm{X}$ accelerator complex is shown in Figure 1.6. This facility is based on high intensity $\mathrm{H}^{-}$linear accelerator. It will operate at continuous wave $(\mathrm{CW})$ mode. The schematic of baseline configuration of the proposed $3 \mathrm{GeV}$ superconducting $\mathrm{CW}$ linac is shown in Figure 1.7. Linac is capable to accelerate an average beam current of $1 \mathrm{~mA}$ (average over $>1 \mu \mathrm{s}$ ) and pulse peak beam current of $10 \mathrm{~mA}$ (average over $<1 \mu \mathrm{s})$ corresponding to a frequency of $325 \mathrm{MHz}$. As shown in block diagram (Figure 1.7), ion source $\left(\mathrm{H}^{-}\right.$gun), radio frequency quadrupole (RFQ) and medium energy beam transport (MEBT) system operate at room temperature (RT) and rest of the linac is superconducting. These systems are described as below.

\begin{tabular}{|c|c|c|c|c|c|c|c|}
\hline Hgun & RFQ & MEBT & SSR0 & SSR1 & SSR2 & $\beta=0.61$ & $\beta=0.9$ \\
\hline
\end{tabular}

Figure 1.7: Acceleration scheme for $3 \mathrm{GeV}$ SC CW linac of the Project-X. 
Ion source: Ion source for $\mathrm{CW}$ linac is capable of delivering direct current beam of $\mathrm{H}^{-}$ions of magnitude up to $10 \mathrm{~mA}$. A prototype of ion source has been designed and tested at LBNL [21]. Transverse emittance is measured for different currents ranging from $1 \mathrm{~mA}$ to $10 \mathrm{~mA}$ at various beam energies. Normalized root-mean-square (r.m.s) transverse emittances were found less than $0.2 \pi \mathrm{mm}$ mrad, which met the specification for beam requirements. This beam is transported to the upstream of RF quadrupole using low energy beam transport (LEBT) system. It includes diagnostic devices and solenoids. A schematic of LEBT, also consisting of ion source, is shown in Figure 1.8.

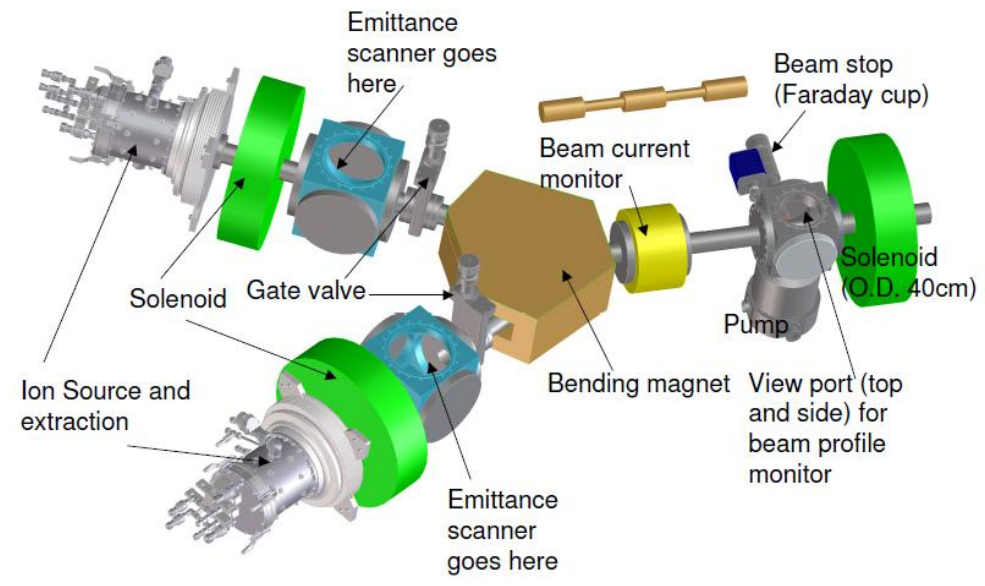

Figure 1.8: Ion source and LEBT-Schematic of beam propagation from ion source to the upstream of RFQ.

RF Quadrupole and Medium energy beam transport system: RFQ is well suited for the acceleration of low velocity beam typically in the range of $\beta=0.01$ to 0.07. It also provides longitudinal bunching and transverse focusing of the beam. RFQ for Project-X CW linac is normal conducting and it operates at $325 \mathrm{MHz}$. It accelerates the beam up to $2.5 \mathrm{MeV}$. The RFQ is followed by MEBT section. Since the linac average beam current is $1 \mathrm{~mA}$ and the beam current at the ion source can be as high as $10 \mathrm{~mA}$, up to $90 \%$ beam has to be removed by chopper in MEBT section. The power of removed beam is quite high, of the order $\sim 25 \mathrm{~kW}$, and thus it will require a dedicated beam dump. The beam energy of $2.5 \mathrm{MeV}$ was chosen in part because it is below the neutron production threshold for most of the materials. Beam chopper provides beam time structure which is necessary to operate the different 


\section{MOTIVATION AND FRAMEWORK: PROPOSED PROJECT-X FACILITY AT FERMILAB}

experiments simultaneously. MEBT operates at room temperature and it consists of matching section which provides beam matching between superconducting and normal conducting section of the linac.

Superconducting (SC) linac: The MEBT is followed by SC linac which is segmented into two sections: low energy part and high energy part. In present design low energy section (2.5-160 MeV) uses three families of SC single spoke resonators (SSR) i.e. SSR0, SSR1 and SSR2 which are operated at $325 \mathrm{MHz}$. The high energy section of the SC linac (160 MeV-3.0 GeV) uses two families of 5-cell, elliptical cavities which are designed for $\beta_{G},=0.61$ and $\beta_{G}=0.9$ where $\beta_{G}$ is the geometrical velocity. These cavities are operated at the frequency of $650 \mathrm{MHz}$.

$3 \mathrm{MW}$ beam at the end of CW linac will be divided between neutrino program and rare decay process program, using a pulsed dipole. Further, a RF beam splitter can deliver the beam to multiple (at least three) experimental facilities (shown in Figure 1.6. For the second stage of acceleration, a pulsed linac is used for the acceleration of 1 $\mathrm{mA}$ beam pulses with $1-5 \%$ duty cycle from $3 \mathrm{GeV}$ to $8 \mathrm{GeV}$. A pulse linac is preferred over rapid cycling synchrotron due to its flexibility for further upgrade. This beam is injected to recycler which delivers $2 \mathrm{MW}$ beam power at any energy between 60 $120 \mathrm{GeV}$ to support neutrino factory. Operation of many experiments simultaneously

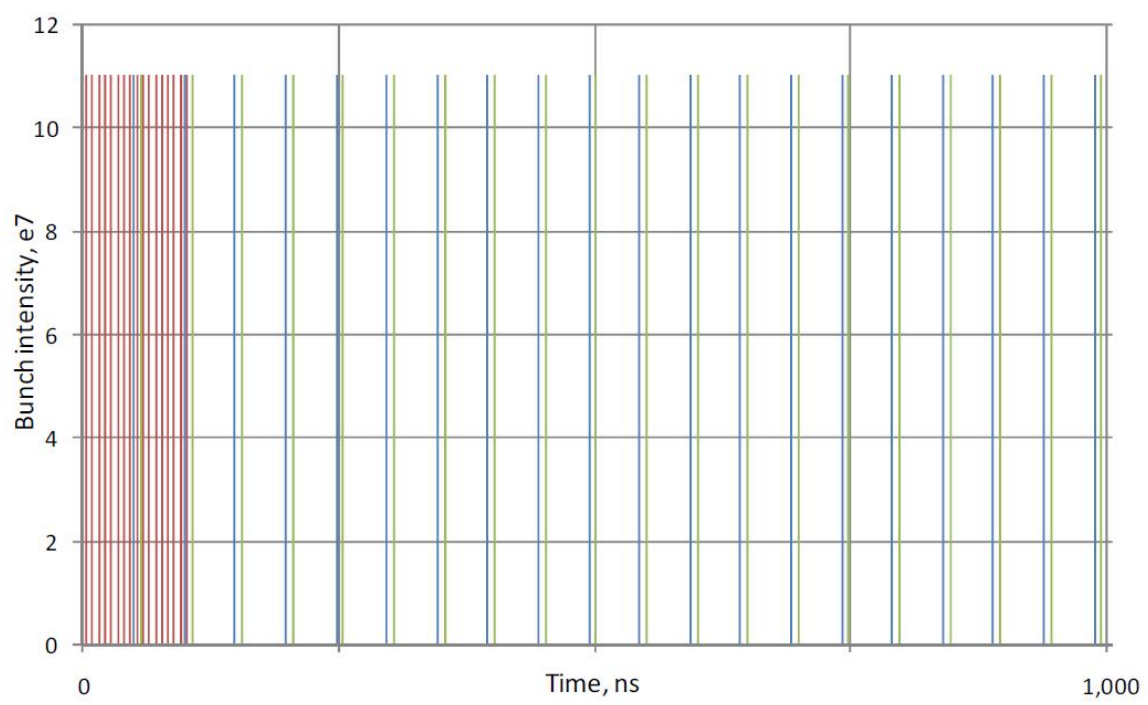

Figure 1.9: A 1- $\mu$ sec period in the $\mathrm{CW}$ linac, with red pulses for the muon conversion experiment, blue for rare kaon decay experiments, and green for other experiments. 
require special beam time line. To achieve required time structure for bunches, broad band chopper [22] removes selective bunches. It reduces beam average current from 10 $\mathrm{mA}$ to $1 \mathrm{~mA}$. There are two time lines associated with beam chopping:

- Timeline for strip injection into the Recycler/Main Injector (MI).

- Timeline for the $3-\mathrm{GeV}$ program.

The injection into the ring requires $1-5 \%$ of the linac duty cycle. The total charge needed for injection is $26 \mathrm{~mA}$-ms for every 0.7-1.4 seconds (determined by the MI ramp cycle). The required bunch chopping during this timeline is associated with the ring $\mathrm{RF}$ frequency $(\sim 53 \mathrm{MHz})$ and with the kicker gap needed every revolution period (11 $\mu s)$. The remainder of the duty cycle $(>95 \%)$ is delivered to the $3-\mathrm{GeV}$ experiments. Distribution of beam in these experiments is not decided yet but a preliminary proposal has been made. The time distribution to different experiments at Project-X facility is shown in Figure 1.9. Using an RF separator running at 1.25 times of the bunch frequency i.e., $406.25 \mathrm{MHz}$, every other pulse is available to the muon experiment, so a burst of $17,162.5-\mathrm{MHz}$ bunches $(\sim 100 \mathrm{nsec})$ of $11 \times 10^{7}$ particles per bunch can be provided. The other RF buckets are chopped and equally split between two other experiments to match the $20-30 \mathrm{MHz}$ desired bunch spacing.

\subsection{Motivation for present work}

The main emphasis of this thesis is on the design of superconducting radio frequency (SCRF) cavities for intermediate and high energy sections of the Project-X linac and on the design of beam transport line (lattice) for CW linac.

The use of an elliptical cavity for particle acceleration has resulted as a consequence of a series of trade-off between different cavity parameters, ranging from RF to mechanics, and takes into account the constraints imposed due to cavity design technique and fabrication experience. It includes both fundamental as well as practical aspects. The fundamental aspects of cavity design comprise of choice of operating frequency, number of cells in multi-cell cavity, operating range of cavity etc. The practical aspects of cavity design include concerns from industrial production and technological constraints such as availability of auxiliary components (tuner, power coupler etc.) 


\section{MOTIVATION AND FRAMEWORK: PROPOSED PROJECT-X FACILITY AT FERMILAB}

Shapes of $\beta_{G}=0.90$ and $\beta_{G}=0.61$ cavities for $\mathrm{CW}$ version of Project-X linac are optimized to achieve maximum acceleration and minimum power dissipation to reduce cryogenic losses. Field enhancement factors (i.e., ratio of peak surface electric and magnetic fields to accelerating fields) are minimized to increase the accelerating gradient while ratio of shunt-impedance to quality factor $(\mathrm{R} / \mathrm{Q})$ and geometrical factor for operating mode are maximized to reduce the power dissipation to the wall of cavity. Optimization of shape and calculation of RF parameters such as field enhancement factors, quality factor, shunt impedance etc. of cavity are performed using two-dimensional cavity design code SLANS. Higher order modes (HOMs) spectrum is studied for both cavities. The cavities are designed such that there are no significantly trapped HOMs. A systematic study is performed to understand the effects of excitation of resonance of HOMs on beam quality and resultant power dissipation. Analysis of HOMs provides better understanding of the requirements of HOM damper for these cavities.

Linac design of Project-X has evolved over recent years. Initial proposal of Fermilab's Projct-X facility, based on a pulsed linac, was considered for the acceleration of beam from kinetic energy of $420 \mathrm{MeV}$ to $1.2 \mathrm{GeV}$ in high energy section. These cavities are designed for $\beta_{G}=0.81$, operating at a frequency of $1.3 \mathrm{GHz}$ and initially included 11-cells to utilize existing design of cryomodule. In this thesis, the end cells of 11-cell cavity are optimized to avoid potentially trapped HOMs. Asymmetric design of end cells are used at both ends so that if any mode gets trapped from one end then it may propagate through other end. Although 11-cell cavity is well optimized but there were few concerns from the fabrication point of view. The presence of 11-cell makes the cavity more sensitive to fabrication errors and thus industrial yield is expected to be low. An alternative study is also performed in this work to design 9-cell cavity for the pulsed linac.

One of the most challenging tasks of Project-X facility is to have a robust design of the CW linac which can provide high quality beam to several experiments simultaneously. Hence a careful design of linac is important to achieve this objective. $\mathrm{H}^{-}$ion is non-relativistic at kinetic energy of $2.5 \mathrm{MeV}$ and its velocity changes very rapidly with acceleration in Project-X linac. Thus, linac uses several types of accelerating structures which are optimized for different particle velocities to provide efficient acceleration. On the basis of energy, linac is segmented into three sections, i.e. low energy section, intermediate energy section and high energy section. Three families of spoke 
resonators are used in low energy section of linac, which are operated at a frequency of $325 \mathrm{MHz}$. These will be used to accelerate the beam from kinetic energy of $2.5 \mathrm{MeV}$ to $160 \mathrm{MeV}$. This is followed by intermediate energy section which consists of 5-cell, elliptically shaped cavities which will be operated at a frequency of $650 \mathrm{MHz}$. These cavities are designed for particles travelling with $61 \%$ of speed of light (geometrical beta $\left.\beta_{G}=0.61\right)$ and will be used to accelerate the beam from kinetic energy of 160 $\mathrm{MeV}$ to $500 \mathrm{MeV}$. High energy section also consists of 5-cell, elliptical cavities which will be operating at a frequency of $650 \mathrm{MHz}$, but, these cavities are designed for $\beta_{G}=$ 0.90 to accelerate the beam from kinetic energy of $500 \mathrm{MeV}$ to $3.0 \mathrm{GeV}$.

Further, a robust lattice is designed for reliable operation of CW linac which preserves beam quality and allows robustness in design parameters such as accelerating gradients in cavities, focusing gradient in magnets etc. Studies are performed for the baseline lattice to analyze beam trajectory and beam emittance in longitudinal and transverse plane using beam tracking codes, TRACEWIN and TRACK. An essential measure of a successful accelerator system is its ability to provide high beam availability and high reliability. The multiuser facility and further upgrade for higher current to test Project-X as a test facility of accelerator driven system requires minimum beam interruption for reliable operation of linac. To improve the reliability of linac, possibility of failure of beam line elements are included in the lattice design. Operation of the linac at $\mathrm{CW}$ mode puts stringent tolerances on beam transport elements, especially at low energy section, which increases the possibility of temporary or permanent loss of accelerating cavities and focusing magnets during the operation of linac. Failure of the beam transport elements like cavity, solenoid and quadrupole alters the focusing period of the beam, resulting in a mismatch of the beam with the subsequent sections. This, in turn, causes beam losses. Sensitivity of the linac performance to failure of elements also depends on the location of failed elements. In some cases, failure of the beam transport element results in huge beam losses and it becomes necessary to replace this element for nominal operation of the machine. To recover nominal performance of linac using traditional way involves replacement of a complete cryomodule (containing several cavities). It is required to warm up the cryomodule from operating temperature (usually $2 \mathrm{~K}$ ) to room temperature and after replacement cryomodule is again cooled down to the operating temperature. Furthermore, procedure to resume the nominal operation is identical as starting procedure and requires slowly ramping up the accelerator. Thus, 


\section{MOTIVATION AND FRAMEWORK: PROPOSED PROJECT-X FACILITY AT FERMILAB}

these beam interruptions reduce the beam availability to the different experiments for a long time. Lattice should be robust enough to have a capability that RF cavity or magnet failure may be compensated locally by using the neighbouring elements. The numbers of cavities and focusing magnets in a cryomodule are chosen in such a way that at least one failure of RF cavity or focusing magnet can be compensated locally.

SCRF cavities are complex and expensive and their fabrications involve lot of scientific and engineering efforts. A standard procedure developed for the fabrication of 1.3 GHz, 9-cell cavity at mass scale, is discussed in this work. Similar approach will be used for the fabrication of $650 \mathrm{MHz}$ cavities for CW linac. Deep-drawing method is commonly used to fabricate niobium cavity. Quality of inner surface of cavity degrades during process of fabrication which may limit maximum accelerating gradient in cavity. Thus, it is passed through sequence of surface treatments in order to remove defects from production process. Surface treatments involve chemical etching (electro chemical polishing, buffer chemical polishing), high pressure water rinsing and heat treatment of cavity. After all treatments, performance parameters of cavity like accelerating gradient and quality factor are tested at $2 \mathrm{~K}$ in the vertical-test stand (VTS) facility.

\subsection{Thesis Organization}

The thesis is organized in six chapters. A brief description of each chapter is given below.

Chapter 1 provides an overview of the proposed high intensity proton facility, Project-X at Fermilab, Batavia, USA. Physics objectives and a layout of Project-X facility are presented.

Chapter 2 reviews basics of beam physics necessary to discuss beam motion and fundamentals of lattice design for high intensity beam. This chapter also includes an electromagnetic description of cavity and its performance parameters such as quality factor, accelerating gradient, shunt impedance etc. In modern high energy accelerator, usually superconducting cavities are preferred over normal conducting cavities. A brief overview of superconductivity, superconducting material for cavity fabrication and their properties are presented.

Chapter 3 addresses the general design criteria for cavity such as choice of frequency and number of cells in a multi-cell cavity. A topology for geometry of elliptical cavity 
is introduced. Influence of geometrical parameters on cavity performance, simulation tools for cavity design and limiting mechanisms in high accelerating gradient cavities are discussed. Methodology behind the design of $1.3 \mathrm{GHz} \beta_{G}=0.81$ cavity for the pulsed variant of $H^{-}$ion linac and $650 \mathrm{MHz}, \beta_{G}=0.9$ and $\beta_{G}=0.61$ cavities for $\mathrm{CW}$ variant of $H^{-}$linac for the Project-X facility are presented. Higher order modes (HOM) coupler is an expensive component of accelerating structure. In order to determine the requirement of $\mathrm{HOM}$ coupler for $650 \mathrm{MHz}$ cavities, analysis is performed to estimate power losses and emittance growth due to HOM in intermediate and high energy sections of CW linac.

Chapter 4 presents architecture of CW linac for the Project-X facility. Choice of accelerating structures, magnets, assumptions and limitations used for lattice design are discussed. Beam quality parameters such as emittance and halo parameters are analyzed for baseline lattice. Longitudinal and transverse acceptances are studied. Operation of linac in CW mode puts stringent tolerances on beam line elements especially at low energy. Failures of beam line elements at critical locations are studied and their local compensation scheme is demonstrated.

Chapter 5 introduces fabrication techniques for SRF cavity in context of fabrication of $1.3 \mathrm{GHz}, \beta_{G}=1$ cavity. Fabrication processes include building of half cells from raw material sheets, electron beam welding to form single cell or dumbbells. Various techniques are described for surface cleaning such as high pressure water rinsing and post surface purification method such as baking. A single cell $1.3 \mathrm{GHz}, \beta_{G}=1$ cavity is tested on VTS and results are presented.

Chapter 6 summarizes the main features from all the preceding chapters. 
1. MOTIVATION AND FRAMEWORK: PROPOSED PROJECT-X FACILITY AT FERMILAB 


\section{2}

\section{Fundamentals of radio frequency cavity \& beam dynamics}

The radio frequency $(\mathrm{RF})$ based technology has become an integral part of modern high energy accelerators. RF cavity is a key component of the accelerators which completes its meaning of acceleration. An accelerating RF cavity stores electromagnetic energy and delivers it to the charged particles in the form of kick in the direction of motion and hence increase the kinetic energy of particles. This chapter provides an overview of electromagnetic description of cavity and its performance parameters such as quality factor, accelerating gradient, shunt impedance etc. Comparison between superconducting radio frequency (SCRF) cavity and normal conducting cavity is discussed and general characteristics of superconductivity such as Meissner-Ochsenfeld effect, superconductivity in microwave fields, surface resistance, residual resistance etc. are also reviewed. Niobium $(\mathrm{Nb})$ exhibits most attractive $\mathrm{SC}$ properties for RF cavities and it is generally used for building high gradient SCRF cavities. Properties of Nb and alternative SC materials for fabrication of RF cavities are presented. Simulation tools are used to solve eigenvalue problem for various geometries of RF cavities. A brief overview of numerical methods for determining electromagnetic fields in cavity and description of 2-D cavity simulation tool, SLANS is presented. This chapter also reviews basic beam dynamics. Equations of motion of accelerating charged particle in longitudinal and transverse planes are discussed. High intensity effects such as space charge effects and beam halo formation are also addressed. Finally, a description of beam dynamics codes such as TRACEWIN, which are used to design beam transport 


\section{FUNDAMENTALS OF RADIO FREQUENCY CAVITY \& BEAM DYNAMICS}

line, is presented.

\subsection{Electromagnetic description of RF cavity}

A resonant cavity is a volume enclosed by conducting walls that supports electromagnetic (EM) oscillation. In accelerator application, usually cavity supports electromagnetic oscillation in radio frequency regime $(>300 \mathrm{MHz})$. Thus it is also called RF cavity. The EM waves in cavity satisfy Maxwell's equations [23].

\subsubsection{Maxwell's equations}

In charge and current free space, Maxwell's equations in differential form are expressed as follows:

$$
\begin{aligned}
\nabla \cdot \vec{D} & =0 \\
\nabla \cdot \vec{B} & =0 \\
\nabla \times \vec{E} & =-\frac{\partial \vec{B}}{\partial t} \\
\nabla \times \vec{H} & =\frac{\partial \vec{D}}{\partial t},
\end{aligned}
$$

where $\vec{E}$ and $\vec{H}$ are electric field intensity and magnetic field intensity respectively. $\vec{D}$ and $\vec{B}$ are electric displacement field and magnetic field respectively which can be expressed in terms of $\vec{E}$ and $\vec{H}$ as following:

$$
\begin{aligned}
\vec{D} & =\epsilon \vec{E} \\
\vec{B} & =\mu \vec{H} .
\end{aligned}
$$

$\epsilon$ is permittivity and $\mu$ the permeability of the material in which the fields exist. In a linear, isotropic, non-dispersive, uniform material $\epsilon$ and $\mu$ are scalar constants and expressed as:

$$
\begin{aligned}
\epsilon & =\epsilon_{r} \epsilon_{0} \\
\mu & =\mu_{r} \mu_{0}
\end{aligned}
$$

where $\epsilon_{r}$ and $\mu_{r}$ are the material dependent constants and they have unit magnitude in free space. $\epsilon_{0}$ and $\mu_{0}$ are universal constants, which obey the following relation:

$$
c=\frac{1}{\sqrt{\epsilon_{0} \mu_{0}}}
$$


where $c$ is speed of light in free space. Maxwell's equations can be combined to yield the wave equation which is given as :

$$
\left(\nabla^{2}-\frac{1}{c^{2}} \frac{\partial^{2}}{\partial t^{2}}\right)\left\{\begin{array}{l}
\vec{E} \\
\vec{H}
\end{array}\right\}=0
$$

Solution of wave equation in terms of plane wave in cylindrical coordinates $(\rho, \phi, z)$ can be written as:

$$
\begin{aligned}
\vec{E}(\vec{r}, t) & =\vec{E}(\rho, \phi) e^{i(k z-\omega t)} \\
\vec{H}(\vec{r}, t) & =\vec{H}(\rho, \phi) e^{i(k z-\omega t)}
\end{aligned}
$$

where $\omega$ is the angular frequency and $k$ is wave number.

\subsubsection{Cylindrical waveguide}

RF source feeds power to the infinite cylindrical waveguide with circular cross-section bounded by a perfect conductor. Thus, it excites electromagnetic fields in waveguide. On the surface of a perfect conductor the following boundary conditions are applied:

$$
\vec{n} \times \vec{E}=0, \vec{n} \cdot \vec{H}=0,
$$

where $\vec{n}$ is the unit vector normal to the surface of the conductor. Substituting the fields from equation (2.8) into equation (2.7), simplified wave equation can be written as:

$$
\left(\nabla_{\perp}^{2}+\gamma_{j}^{2}\right)\left\{\begin{array}{l}
\vec{E} \\
\vec{H}
\end{array}\right\}=0
$$

where

$$
\nabla_{\perp}^{2}=\nabla^{2}-\frac{\partial^{2}}{\partial z^{2}}
$$

The solutions to the wave equation 2.10 form an orthogonal set with $j^{\text {th }}$ eigenvalues $\gamma_{j}^{2}=\omega^{2} / c^{2}-k^{2}$. Maxwell's equations can be combined to express the transverse fields $\left(E_{\perp}, H_{\perp}\right)$ in the waveguide as a function of the longitudinal component of fields $\left(E_{z}, H_{z}\right)$ [23. The different boundary conditions imposed on $E_{z}$ and $H_{z}$ and the fact that $E_{z}$ and $H_{z}$ are independent imply that the solution to equation 2.10 forms two sets of modes, generally with different eigenvalues. The two families are denoted as transverse magnetic (TM) modes and transverse electric (TE) modes. For TM modes, $E_{z}$ exists whereas $\vec{H}$ is transverse every where. Conversely, for TE modes, $H_{z}$ exists 


\section{FUNDAMENTALS OF RADIO FREQUENCY CAVITY \& BEAM DYNAMICS}

and $\vec{E}$ is transverse every where. The modes are classified by the nomenclature $\mathrm{TM}_{m n}$ or $\mathrm{TE}_{m n}$ where indices $m$ and $n$ are measures of the number of sign changes of $E_{z}$ in the $\phi$ and $\rho$ directions, respectively. In both cases, transverse magnetic fields and electric fields are related by:

$$
\vec{H}_{\perp}= \pm \frac{\hat{z} \times \vec{E}_{\perp}}{Z}
$$

where

$$
Z_{T M}=\frac{k}{\epsilon_{0} \omega}
$$

is the wave impedance for TM modes and

$$
Z_{T E}=\frac{\mu_{0} \omega}{k}
$$

for TE modes. The positive case applies to waves traveling in the $+\hat{z}$ direction i.e., forward traveling wave while negative case refers to backward traveling waves. The transverse component of fields can be derived from longitudinal component as given below:

$$
\begin{aligned}
& \vec{E}_{\perp}= \pm \frac{i k}{\gamma_{j}^{2}} \nabla_{\perp} E_{z} \quad(\mathbf{T M} \text { modes }) \\
& \vec{H}_{\perp}= \pm \frac{i k}{\gamma_{j}^{2}} \nabla_{\perp} H_{z} \quad(\mathbf{T E} \text { modes })
\end{aligned}
$$

where $E_{z}$ and $H_{z}$ satisfy equation 2.10$)$. However, this traveling wave solution is valid for waveguide only.

\subsubsection{Cylindrical cavity}

A cavity can be considered as a waveguide with conducting faces at both ends i.e., at $z=0$ and $z=d$. Standing waves are created due to reflections at both ends. Imposing this additional boundary condition, eigenvalue of equation 2.10 for discrete value of $k=p \pi / d$ can be expressed as:

$$
\gamma_{j}^{2}=\left(\frac{\omega_{j}}{c}\right)^{2}-\left(\frac{p \pi}{d}\right)^{2} \quad p=0,1,2 \cdots
$$

Cylindrical resonant cavity or pill-box cavity is simplest model to estimate field distribution in a resonant cavity. Consider a pill box cavity of length $d$ and radius $R$. The solutions of eigenvalue equation (2.10) are Bessel functions. The modes in cavities are classified by the nomenclature $\mathrm{TM}_{m n p}$ or $\mathrm{TE}_{m n p}$ where additional index $p$ measures the 
number of sign changes of $E_{z}$ in $z$ direction. TM mode with lowest resonant frequency is $\mathrm{TM}_{010}$ mode. The fields corresponding to this mode are:

$$
\begin{aligned}
E_{z} & =E_{0} J_{0}\left(\frac{2.405 \rho}{R}\right) e^{i \omega_{010} t} \\
H_{\phi} & =-i \frac{E_{0}}{\eta} J_{1}\left(\frac{2.405 \rho}{R}\right) e^{i \omega_{010} t},
\end{aligned}
$$

and all other field components are vanished. $\eta=\sqrt{\mu_{0} / \epsilon_{0}}, J_{0}$ and $J_{1}$ are zeroth and first order Bessel functions, respectively. The resonant frequency corresponding to this mode is given by:

$$
\omega_{010}=\frac{2.405 c}{R},
$$

It is independent of the length of cavity. Fields for TE modes can be solved similarly. TE modes have no longitudinal electric fields and thus can not be used for acceleration of the charged particles beam. Conversely, beam along the axis can not excite these modes.

An accelerating cavity is designed to operate at particular frequency which is excited using external RF source. However, cavity resonates not only in accelerating mode but also in other modes. Operating mode of acceleration is usually chosen with lowest frequency i.e., $\mathrm{TM}_{010}$ mode which is chosen because of uniform longitudinal electric field $\left(\partial E_{z} / \partial z=0\right)$. Other modes with higher frequencies are called higher order modes (HOMs). There are different families of HOMs such as TE and TM modes of different rotational symmetries (monopole, dipole, quadrupole etc.). When beam traverses through the cavity, it excites HOMs. All modes of the type $\mathrm{TM}_{0 n p}$ are called monopole modes or longitudinal modes because of their uniform azimuthal fields distribution. Monopole modes (other than $\mathrm{TM}_{010}$ ) can cause increase in power dissipation in RF cavity which leads to increased cryogenic losses. Modes of the type $\mathrm{TM}_{1 n p}$ have a net deflecting field on axis. These kind of modes are called dipole modes. These modes are undesirable in cavity as they deflect the beam which may result in many beam dynamics issues. Thus, it is necessary to damp HOMs in cavity. These are the dominating factors influencing the design of high current cavities. Therefore, study of HOM spectrum is one of the main features of cavity design. One can also use HOM damper (HOM coupler) to extract HOMs. 


\section{FUNDAMENTALS OF RADIO FREQUENCY CAVITY \& BEAM DYNAMICS}

\subsection{Multi-cell RF cavities}

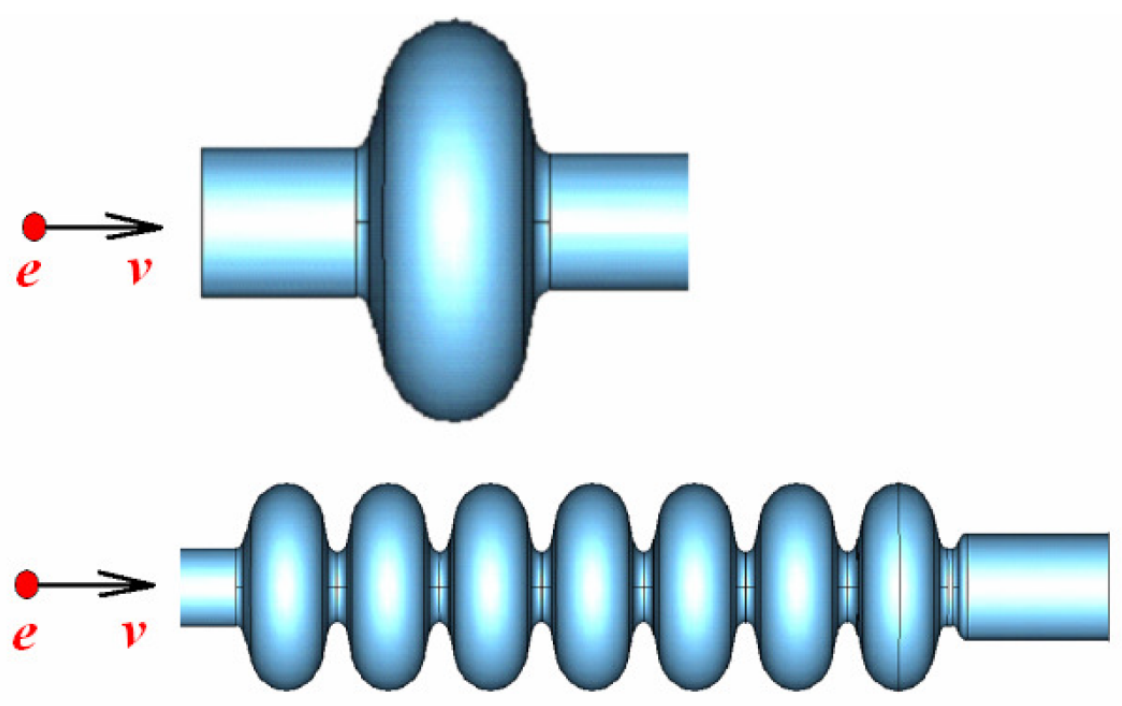

Figure 2.1: Single cell (top) and multi-cell (bottom) cavity with beam pipe.

In high energy accelerators, RF cavity is a chain of resonators (Figure 2.1) which are coupled with each other through a hole called "iris". Each coupled resonator is called cell and cavity is called multi-cell cavity. Single cell cavity is often used in circular accelerator to simplify damping scheme for higher order modes. It is also preferred for non relativistic beam due to its high velocity acceptance. Fabrication and surface processing are also easy for single cell cavity and there is no issue of field flatness. However, energy gain per cavity is proportional to number of cells in a multi-cell cavity. The main advantages of multi-cell cavity are:

- A well optimized $N$-cell cavity requires single RF source to feed power into cavity instead of $N$ RF sources for $N$ single-cell cavities to achieve same energy gain. Thus, choice of multi-cell cavity reduces number of power feeds which helps to reduce the cost of accelerator.

- Energy gain is proportional to number of cells in a cavity. Thus, multi-cell option helps to achieve a possible saving of space in beam transport line.

- Use of multi-cell cavity also reduces number of auxiliary components (HOM damper, tuner etc.) required. 
- Multi-cell alternative allows use of larger power supply.

A multi-cell standing wave cavity can be represented as a system of coupled oscillators. As we know that a system of connected mechanical pendulums can oscillate at different modes with different frequencies, similarly in a multi-cell cavity with $N$ cells, a single cell mode splits into $N$ different multi-cell modes and formed a passband. Each of them has different frequency and different phase shift between adjacent cells. The frequencies of coupled modes are given as:

$$
f_{m}=f_{0} / \sqrt{1+2 k \cos \left(\frac{\pi \cdot m}{N}\right)} \quad 1 \leq m \leq N,
$$

where $f_{m}$ is frequency of the $\mathrm{m}^{t h}$ mode, $N$ is the number of cells in multi-cell cavity, $f_{0}$ is the resonant frequency and $k$ measures how strongly two cells are coupled with each other and is known as cell to cell coupling coefficient.

Usually for efficient acceleration SCRF cavities are operated at $\pi$ mode. It means that phase difference between adjacent cells is $180^{\circ}$ as shown in Figure 2.2.

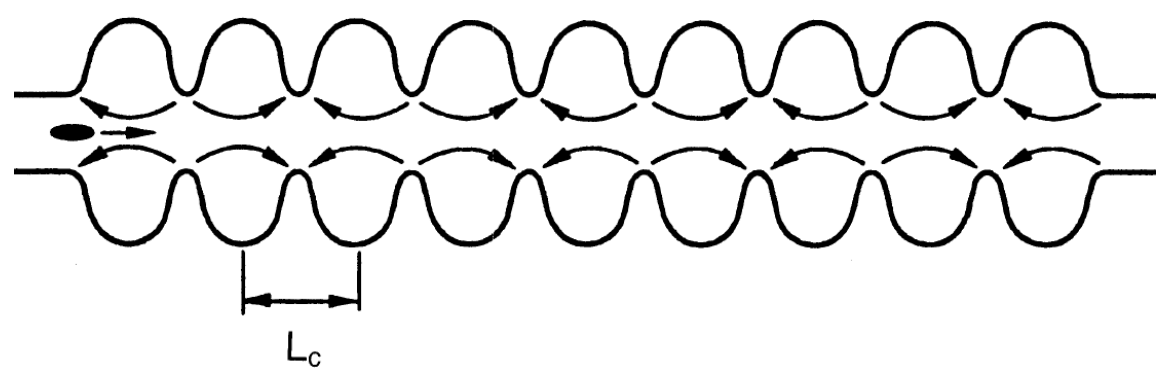

Figure 2.2: $\pi$ mode field distribution in multi-cell cavity - $180^{\circ}$ phase advance of $\mathrm{RF}$ wave from cell to cell.

The cell length $L_{c}$ is determined by the condition that the electric field has to be inverted in the time a charge particle needs to travel from one cell to the next. Thus, field always points in the same direction while particle traverses the cavity. From the condition of $180^{\circ}$ phase advance per cell, particle with matched velocity spends only half of a RF period to cross one cell of cavity. Thus length of a single cell $\left(L_{c}\right)$ is given as:

$$
L_{c}=\frac{1}{2} \beta_{G} \lambda
$$




\section{FUNDAMENTALS OF RADIO FREQUENCY CAVITY \& BEAM DYNAMICS}

where $\lambda$ is RF wave length and $\beta_{G}$ is geometrical beta for which particle is in perfect synchronism with RF wave. Total length of cavity for $N$ - cell is:

$$
L_{c a v}=\frac{1}{2} N \beta_{G} \lambda
$$

\subsection{Performance parameters of a cavity}

Cavity performance is characterized on the basis of quantities such as stored energy, accelerating gradients, power dissipation, quality factors, shunt impedance etc. It is necessary to understand these quantities prior to cavity design in order to determine optimal shape and size of cavity which satisfy operation requirements. This section addresses these quantities of cavity performance.

\subsubsection{Stored energy}

Cavity stores EM energy which is utilized to accelerate charge particle beam. The electromagnetic energy density is

$$
U_{d}=\frac{1}{2} \vec{D} \cdot \vec{E}+\frac{1}{2} \vec{B} \cdot \vec{H},
$$

and thus total energy stored in a cavity is:

$$
U=\int_{V} U_{d} d V
$$

where $V$ is the volume enclosed by RF cavity.

\subsubsection{Peak surface fields}

Stored energy in a cavity is limited by the maximum surface fields that cavity can maintain. We therefore introduce two fields, the peak surface electric field $\left(E_{p k}\right)$ and the peak surface magnetic field $\left(H_{p k}\right)$. In normal conducting cavities, very high peak surface electric field can result in electrical breakdown. For SCRF cavities, high electric field can result in field emission (FE) which produces electrons inside the cavity that absorbs RF energy and create unnecessary power losses. Thus, SCRF cavities are operated below this $\mathrm{FE}$ region.

The surface magnetic fields correspond to surface current in a cavity. Thus, peak surface magnetic field in normal conducting cavity is limited by cooling requirements 
while in SCRF cavities peak surface magnetic fields are restricted by RF critical magnetic field. The peak surface magnetic field can not exceed RF critical magnetic field otherwise superconductor will quench to normal conducting state.

\subsubsection{Accelerating gradient}

When a charged particle travels through the RF cavity, it interacts with EM wave over the distance $L_{c a v}$ (length of cavity) and gains energy. The energy gain across the cavity can be written in term of accelerating voltage $V_{a}$ :

$$
V_{a}=\mid \frac{1}{e} \times \text { energy gain during passage through cavity } \mid
$$

i.e., $V_{a}$ is given by line integral of longitudinal electric field $\left(E_{z}\right)$ as seen by charged particle crossing the cavity along the axis $(\rho=0)$ with relative velocity $\beta$.

$$
V_{a}=\left|\int_{0}^{L_{c a v}} E_{z}(\rho=0, z) e^{i \omega_{0} z / \beta c} d z\right|
$$

where $\omega_{0}$ is angular operating mode frequency of RF cavity. Thus, the average accelerating electric field $\left(E_{a c c}\right)$ that charged particle sees during transit is given by:

$$
E_{a c c}=\frac{V_{a}}{L_{c a v}}
$$

\subsubsection{Transit time factor}

The charge particle takes finite time to traverse through a cavity. Therefore, energy gain of a particle passing through harmonically time varying field is different than when it passes through in a static field. The ratio of energy gain of charge particle in a time varying field to energy gain in static field is termed as transit time factor (TTF). It can be expressed as:

$$
T T F=\frac{\int_{-L_{c a v} / 2}^{L_{c a v} / 2} E_{z}(\rho=0, z) e^{i \omega_{0} z / \beta c} d z}{\int_{-L_{c a v} / 2}^{L_{c a v} / 2} E_{z}(\rho=0, z) d z},
$$

TTF measures the energy range in which a cavity can work efficiently.

\subsubsection{Quality factor}

The Quality factor $(Q)$ of a cavity is directly related to power lost in the cavity. It is defined as $2 \pi$ times of number of RF cycles it takes to lose total energy stored in 


\section{FUNDAMENTALS OF RADIO FREQUENCY CAVITY \& BEAM DYNAMICS}

the cavity or $2 \pi$ times the ratio of energy stored in the cavity to the energy lost per RF period. Quantitatively, the unloaded quality factor or wall $Q$ can be written for operating mode as:

$$
Q_{0}=\frac{\omega_{0} U}{P_{d}},
$$

where power losses is considered only due to unavoidable Joule heating of cavity wall. Power dissipated $\left(P_{d}\right)$ to the inner surface of cavity wall is given as:

$$
P_{d}=\frac{1}{2} R_{s} \int_{s}|\vec{H}|^{2} d s
$$

where integration is taken over the interior surface of the cavity. It is assumed that inner surface resistance $R_{s}$ does not vary over the cavity surface. Since $P_{d}=d U / d t$, time evolution of stored energy in the absence of RF source and beam is given by:

$$
U(t)=U(t=0) e^{\frac{-\omega_{0} t}{Q_{0}}}
$$

Hence the time it takes for EM energy to decay to $1 / e$ of its initial value is:

$$
t_{f}=\frac{Q_{0}}{\omega_{0}} \quad \text { decay time of stored energy }
$$

which is filling time in standing wave structure. There are other factors which contribute to power losses in the RF cavity. The beam passing through cavity consumes RF power. It is also leaked out of the cavity through the opening of power coupler and HOM coupler. Thus, in the absence of beam, total RF power losses $\left(P_{\text {total }}\right)$ in the cavity can be expressed as sum of external power losses $\left(P_{e x}\right)$ and wall power losses.

$$
\begin{aligned}
& P_{\text {total }}=P_{t}+P_{r}+P_{d} \\
& P_{\text {total }}=P_{e x}+P_{d}
\end{aligned}
$$

where $P_{t}$ and $P_{r}$ are power transmitted out and power reflected back respectively. If $P_{d}$ is replaced by $P_{\text {total }}$ in equation 2.30 then we get loaded quality factor, represented as $\mathrm{Q}_{L}$. The relation between unloaded quality factor and loaded quality factor can be 
obtained by dividing both sides of equation 2.35 by $\omega_{0} U$ :

$$
\begin{aligned}
\frac{P_{\text {total }}}{\omega_{0} U} & =\frac{P_{e x}}{\omega_{0} U}+\frac{P_{d}}{\omega_{0} U} \\
\frac{1}{Q_{L}} & =\frac{1}{Q_{e x}}+\frac{1}{Q_{0}} \\
Q_{L} & =\frac{Q_{0}}{1+\beta_{c}},
\end{aligned}
$$

where

$$
\beta_{c}=\frac{Q_{0}}{Q_{e x}}=\frac{P_{e x}}{P_{d}}
$$

$\beta_{c}$ is called coupling coefficient. $\beta_{c}=1$ is called critical coupling and all the power from transmission line goes to resonators and no reflection occurs. $\beta_{c} \ll 1$ is weak coupling case and $\beta_{c} \gg 1$ is strong coupling case.

\subsubsection{Shunt impedance}

Shunt impedance of a cavity is another important quantity which measures effectiveness of producing an accelerating voltage $V_{a}$ for a given power dissipation $P_{d}$ in the cavity. It is defined by:

$$
R=\frac{V_{a}^{2}}{P_{d}}
$$

\subsubsection{R/Q}

$R$ over $Q$ measures the efficiency of acceleration per unit stored energy for the operating frequency. It is an important parameter from cavity design point of view as it is independent of the surface properties that determine power losses and is only a function of cavity geometry. Thus, it is also called geometric shunt impedance or effective impedance of the cavity. For operating mode it is defined as:

$$
\frac{R}{Q_{0}}=\frac{V_{a}^{2}}{\omega_{0} U} .
$$

Effective impedance allows comparison of effectiveness of different cavity geometries and structures. A cavity can be excited at different frequencies corresponding to different modes of oscillations, other than operating mode frequency $\left(\omega_{0}\right)$. As mentioned in subsection (2.1.3), these high frequency modes are HOMs. These can be excited by the 


\section{FUNDAMENTALS OF RADIO FREQUENCY CAVITY \& BEAM DYNAMICS}

bunched beam passing through the cavity. More power can be transferred to the fields of HOMs for higher beam current. These parasitic modes can destroy the bunch. The parameter $R / Q$ is used to determine the level of HOMs excitation by characterizing beam power deposited into HOMs excited by the beam traversing across the cavity. An offset beam can excite transverse modes which are characterized by transverse impedance. It can be expressed as:

$$
\left(\frac{R}{Q}\right)_{\perp}=\left(\frac{R}{Q}\right)_{\|} \cdot \frac{1}{k}
$$

and measured in $\mathrm{Ohm} / \mathrm{cm} . k=\omega / \mathrm{c}$ is wave number and $(R / Q)_{\|}$is given by:

$$
\left(\frac{R}{Q}\right)_{\|}=\frac{\left(\int_{\infty}^{\infty}\left(\frac{\partial E_{z}(x, 0, z)}{\partial x}\right)_{x=x_{0}} e^{\iota k z} d z\right)^{2}}{\omega U} .
$$

and measured in $\mathrm{Ohm} / \mathrm{cm}^{2}$.

\subsubsection{Geometrical factor}

Geometrical factor is commonly used to compare power dissipation of different geometries. It is another figure of merit of cavity which measures cryogenic losses in SCRF cavities and is a function of cavity geometry. It is defined as product of surface resistance and quality factor of the cavity, and is given by:

$$
G=R_{s} Q
$$

From equation 2.40, 2.41) and 2.44, we can obtain:

$$
P_{d}=\frac{R_{s} V_{a}^{2}}{\left(\frac{R}{Q}\right) \cdot G}
$$

Thus, to reduce the power dissipation in RF cavity, it is required to optimize the product of geometrical factor and effective impedance.

\subsection{Why superconducting (SC) cavity?}

When beam passes through cavity, it extracts energy. Thus, one needs to modify equations 2.35 and 2.38 to estimate $P_{\text {total }}$ and $Q_{L}$ respectively, in cavity in presence 
of beam. The RF power needed to establish a certain accelerating voltage is determined mainly by the resistivity of the wall material and beam. Thus, equation 2.35 can be written as:

$$
P_{\text {total }} \sim P_{\text {beam }}+P_{d}
$$

Using equation (2.45), it becomes:

$$
P_{\text {total }} \sim V_{a} . I+\frac{V_{a}^{2}}{\left(\frac{R}{Q}\right) \cdot G} \cdot R_{s}
$$

where $I$ is beam current and $V_{a}$ is accelerating voltage in a cavity. Surface resistance for the normal conductor is of the order of $10^{-3} \Omega$ whereas in superconductor it is of the order of $10^{-9} \Omega$. Thus, for the same geometry (i.e., geometrical factor and $R / Q$ are same) power dissipated in normal conductor will be roughly $10^{5}$ times higher than superconductor. However, SC option is not as efficient as it seems. It is also necessary to account for the cryogenic power $\left(P_{\text {cryo }}\right)$ for real comparison with normal conducting cavity. Cryogenic power is required to maintain low temperature for preserving SC state of cavity. The Carnot efficiency $\left(\eta_{c}\right)$ of refrigerator system is expressed as:

$$
\eta_{c}=\frac{T}{300-T},
$$

where $T$ is operating temperature in cavity, usually $2 \mathrm{~K}$ or $4.2 \mathrm{~K}$. Required cryogenic power is:

$$
P_{\text {cryo }}=\frac{P_{d}}{\eta_{c} \cdot \eta_{t}},
$$

where $\eta_{t}$ is technical efficiency of the system and its typical value is 0.2 to 0.3 . Even including cryogenic power, SC option is much efficient than normal conducting cavity. Since power dissipation is proportional to square of accelerating gradient, possible highest accelerating gradient can be achieved only with SC cavity. Advantages of SCRF cavity can be summarized as following:

- CW or long pulse operation: SCRF cavities are suitable for application demanding high voltage in CW or long pulse regime such as storage ring while in the normal conducting case, the gradient is limited by the difficulties of the remaining heat, produced by the RF losses. 


\section{FUNDAMENTALS OF RADIO FREQUENCY CAVITY \& BEAM DYNAMICS}

- Efficient alternating current (AC) power to beam power transfer: Since power dissipation is lower for SCRF cavity, it has higher efficiency of AC to RF beam power conversion. This efficiency can further increase with increase in beam current.

- Freedom of cavity design: As power dissipation is not a major concern for SCRF cavities, its design can be adopted on the basis of requirements of application such as :

- HOMs can be removed more easily in SCRF cavity with large iris radius. It provides beam stability and allows high energy application.

- Beam interaction with cavity is inversely proportional to iris radius. Thus, large iris radius reduce beam interaction with HOMs. Beam quality can be preserved and it is very important to preserve high quality of beam for the application such as free electron laser (FEL).

- Higher inter cell to cell coupling can be achieved in multi-cell cavity with large iris radius which improves field flatness and hence net acceleration in cavity.

\subsection{Fundamentals of superconductivity}

The term superconductivity was introduced after discovery of phenomenon of zero electrical resistance occurring in certain metals below a characteristic temperature also called critical temperature $\left(\mathrm{T}_{c}\right)$. It was discovered by Dr. Heike Kamerlingh Onnes at University of Leiden in Netherlands when he found that ohmic resistance of mercury reduced to zero at $4.2 \mathrm{~K}$ temperature. After this discovery, It is found that superconductivity is quite a common phenomenon which could be exhibited by several pure elements, many compounds and alloys. Superconductors are extensively used in many applications these days. They have become an integral part of modern high energy accelerators. SC magnets and SCRF cavities are primary choice for high energy accelerators.

Meissner-Ochsenfeld effect: W. Meissner and R. Ochsenfeld discovered in 1933 that when a SC material is cooled down below the $\mathrm{T}_{c}$, any external magnetic field up to a critical magnetic field $\mathrm{B}_{c}$ is expelled. This phenomenon of spontaneous exclusion 
of magnetic field upon crossing temperature below $\mathrm{T}_{c}$ is named as Meissner-Ochsenfeld effect. The field exclusion from bulk superconductor is explained in terms of induced current. When weak magnetic field (lower than $\mathrm{B}_{c}$ ) is applied, induced current is set up near the surface of superconductor. Magnetic field is generated by induced current which cancels external magnetic field within the bulk of superconductor. Magnetic field stronger than $\mathrm{B}_{c}$ (critical magnetic field) results in quenching of the SC state to the normal conducting state. The Meissner-Ochsenfeld effect verified that SC state corresponds to a new thermodynamics phase. Like any thermodynamic phase, the SC state is only stable within special ranges of relevant variables. For the SC state such variables (among others) are mainly temperature and magnetic field. Material like lead makes a phase transition from the normal to the SC state when it is cooled down below $\mathrm{T}_{c}$ and magnetic field is less than $\mathrm{B}_{c}$. SC state is stable only for temperature below $\mathrm{T}_{c}$ and $\mathrm{B}_{c}$. Relationship between these parameters can be expressed as:

$$
B_{c}(T)=B_{c}(0)\left[1-\left(\frac{T}{T_{c}}\right)^{2}\right],
$$

where $\mathrm{B}_{c}(\mathrm{~T})$ and $\mathrm{B}_{c}(0)$ are critical magnetic fields at temperatures $\mathrm{T}$ and $0 \mathrm{~K}$ respectively.

\subsubsection{Type I and Type II superconductors}

Superconductors are classified into two families named as Type I and Type II superconductors. They have certain common features, but differ also in some important ways. The elements lead, mercury, tin, aluminium and many other metals are Type I superconductors. They do not allow to penetrate magnetic flux into the bulk material for the applied magnetic field which stays below $\mathrm{B}_{c}$. Thus, these follow Meissner-Ochsenfeld effect. If the applied magnetic field exceeds a critical value in a superconductor of Type I, SC state breaks down and the normal conducting state is restored.

All SC alloys like lead-indium, niobium-titanium, niobium-tin and the element niobium belong to the large class of Type II superconductors. They are characterized by two critical fields, $B_{c 1}$ and $B_{c 2}$. Below $B_{c 1}$ these are in the Meissner phase with complete field expulsion while in the range $B_{c 1}<B<B_{c 2}$ they enter in the mixed phase in which the magnetic field pierces the bulk material in the form of flux tubes with the normal conducting core. These flux tubes are called vortices or fluxoids. It means 


\section{FUNDAMENTALS OF RADIO FREQUENCY CAVITY \& BEAM DYNAMICS}

that Gibbs free energy starts to increase with penetration of magnetic field into superconductor. Superconductivity is completely destroyed at field above the $B_{c 2}$. Higher critical field for Type II superconductors, makes them more favourable for practical applications.

\subsubsection{Characteristics lengths and distinction between Type I and Type II superconductor}

In 1935 London brothers proposed a phenomenological explanation of Meissner-Ochsenfeld effect of field expulsion at temperature below $\mathrm{T}_{c}$ which can not be explained in term of Maxwell's equations of classical electrodynamics. According to London theory, even in a Type I superconductor the magnetic field is not completely expelled, but penetrates into the material over a small distance, as otherwise the shielding current density would have to be infinitely large. The extent of penetration of magnetic field in superconductor is measured in term of London penetration depth $\left(\lambda_{L}\right)$. It is given by the characteristic length of the exponential decay of the magnetic field into the superconductor.

$$
\begin{aligned}
B(x) & =B(0) \cdot e^{\left(-\frac{x}{\lambda_{L}}\right)} \\
\lambda_{L} & =\sqrt{\frac{m}{\mu_{0} n_{s} e^{2}}},
\end{aligned}
$$

where $e$ is the charge of an electron, $m$ is its mass and $n_{s}$ the number of SC charge carriers per unit volume. A typical value for the penetration depth in niobium is 32 $\mathrm{nm}$ [24]. This theory is not applicable in tne presence of impurities in the material or for a temperature dependence of the penetration depth.

Gorter and Casimir introduced the two-fluid model [25] where a coexistence of a normal and SC fluid of charge carriers are postulated.

$$
n_{c}=n_{n}+n_{s},
$$

where $n_{n}$ and $n_{c}$ are normal conducting charge carrier per unit volume and total charge carrier per unit volume respectively.

The advent of the very successful BCS theory [26] was based on the assumption that electrons begin to condense below Tc to make pairs of electrons, so called Cooper pairs. Two electrons in a pair have opposite momentum and spin. They experience an attractive force mediated via quantized lattice vibrations called phonons. This bound 
state of the two electrons is energetically favourable. As the overall spin of these two paired electrons is zero, many of these pairs can co-exist coherently, just like other bosons. At the boundary between the normal and SC phase the density of cooper pairs does not jump abruptly from zero to its value in bulk but rises smoothly over a finite length called coherence length $\left(\xi_{0}\right)$. Typical value for the coherence length in niobium is around $39 \mathrm{~nm}$ [24]. The relative size of the London penetration depth and coherence length decides if a material is Type I or Type II superconductor. Creation of a boundary means a loss of cooper pair condensation energy in a thickness $\xi_{0}$ but a gain of magnetic energy in a thickness $\lambda_{L}$. There is net energy gain if $\lambda_{L}>\xi_{0}$. The Ginzburg-Landau parameter is introduced to distinguish Type I and Type II superconductivity. It is defined as:

$$
\kappa=\frac{\lambda_{L}}{\xi_{0}}
$$

The criterion for Type I and Type II superconductivity is in summarized as:

$$
\begin{aligned}
& \kappa<\frac{1}{\sqrt{2}} \text { Type-I superconductor } \\
& \kappa>\frac{1}{\sqrt{2}} \text { Type-II superconductor }
\end{aligned}
$$

Niobium has $\kappa \approx 1$ and it is a weak Type II superconductor. The role of impurities on superconductivity was studied by Pippard [27] and was based on the evidence that the penetration depth depends on the mean free path $(l)$ of the electrons in the material. The dependence of characteristics coherence length $(\xi)$ on the mean free path is given as:

$$
\frac{1}{\xi}=\frac{1}{\xi_{0}}+\frac{1}{l}
$$

Pippard also introduced effective penetration depth which is given as:

$$
\lambda_{e f f}=\lambda_{L} \cdot \frac{\xi_{0}}{\xi} .
$$

This relation reflects that the SC penetration depth increases with a reduction in the mean free path. For pure (clean) superconductor $(l \rightarrow \infty)$ one has $\xi=\xi_{0}$. In the case of very impure (dirty) superconductors where $l \ll \xi_{0}$ one has $\xi=l$. The mean free path in the niobium is strongly influenced by interstitial impurities like oxygen, nitrogen and carbon. This point becomes more relevant for SCRF cavities where extremely pure material is required to achieve high accelerating field. 


\section{FUNDAMENTALS OF RADIO FREQUENCY CAVITY \& BEAM DYNAMICS}

\subsubsection{Superconductivity in microwave fields}

Superconductors are free from energy dissipation for direct current (DC) applications, but it is no longer true for alternating current(AC) and particularly not in microwave fields where current changes its sign after every $10^{-9}$ seconds. In this regime, high frequency magnetic field penetrates a thin surface layer and induces oscillation of the electrons which are not bound in Cooper pairs. The power dissipation caused by motion of unpaired electrons can be characterized by a surface resistance $\left(R_{s}^{s c}\right)[28$. Surface resistance of super conductor is composed of two terms as given below

$$
R_{s}^{s c}=R_{0}+R_{B C S}(T) ;
$$

where $\left(R_{B C S}\right)$ is BCS surface resistance which is expressed as:

$$
R_{B C S}=\frac{A}{T} \cdot f^{2} \cdot \exp \left(\frac{-\Delta(T)}{k T}\right),
$$

where $A$ is a constant which depends on material parameters of superconductors such as penetration depth, coherence length, the Fermi velocity and mean free path. Energy required to break cooper pair is $2 \Delta$. It is experimentally observed that below a certain temperature, surface resistance is higher than the BCS prediction. The additional temperature independent term $R_{0}$ is called as residual surface resistance. The operating temperature of SCRF cavity is chosen so that the BCS resistance is reduced to an economically tolerable value. Usually, the optimum working conditions of SCRF cavity are achieved when $R_{s} \approx R_{0}$.

$\mathbf{R F}$ critical field $\left(\mathbf{B}_{s h}\right)$ : For microwave fields, the vortices pass into SC material with a characteristic speed. Any surface barrier being present might be too wide for the vortices to pass within one RF half period. Calculations [29, 30] have shown that the Meissner state can persist even beyond $\mathrm{B}_{c 1}$. The magnetic field above which Meissner state is lost and magnetic flux starts to penetrate into SC material is called super heating critical magnetic field $\left(\mathrm{B}_{s h}\right)$ or $\mathrm{RF}$ critical magnetic field. The superheated field has been calculated on the basis of the Ginzburg-Landau equations. The super heating field is given for different $\kappa$ in reference [31]. 


\subsection{Choice of SCRF cavity material}

A physical limitation of SCRF cavity is that it must be operated in the regime where magnetic field at the inner surface stays below the critical magnetic field of the SC material. In principle, superconductors with high upper critical magnetic field and high critical temperature are considered best for accelerator application but criteria which includes fabrication processes, surface conditions, heat transfer capabilities etc. are also important considerations. For the cavity operated in $\mathrm{CW}$ mode, materials with higher critical temperature and lower surface resistance are chosen to reduce dynamics heat losses.

Niobium is the favourite material in RF superconductivity and accelerator cavity construction. It has highest critical temperature $9.2 \mathrm{~K}$ and the highest magnetic field among all the pure metals. Niobium $(\mathrm{Nb})$ properties are summarized in Table 2.1.

Table 2.1: List of Niobium properties

\begin{tabular}{ccc}
\hline Parameters & Magnitude & Unit \\
\hline Atomic No. & 41 & - \\
Atomic mass & 92.9 & $\mathrm{~g} / \mathrm{mol}$ \\
Density & 8579 & $\mathrm{~kg} \cdot \mathrm{m}^{-3}$ \\
Crystalline Lattice & B.C.C. & \\
Debye temperature & 275 & $\mathrm{~K}$ \\
Melting point & 2741 & $\mathrm{~K}$ \\
Critical temperature & 9.2 & $\mathrm{~K}$ \\
$B_{c 1}(2 \mathrm{~K})$ & $\sim 156$ & $\mathrm{mT}$ \\
$B_{s h}(2 \mathrm{~K})$ & $\sim 230$ & $\mathrm{mT}$ \\
$B_{c 2}(2 \mathrm{~K})$ & $\sim 297$ & $\mathrm{mT}$ \\
Thermal conductivity $(300 \mathrm{~K})$ & 53.7 & $\mathrm{~W} \mathrm{~m}{ }^{-1} K^{-1}$ \\
Electrical resistivity $(300 \mathrm{~K})$ & 14.9 & $\mu \Omega . c m$ \\
\hline
\end{tabular}

Most of high gradient cavities are made of bulk Nb, however, copper cavity whose inner surface is coated with a thin layer of $\mathrm{Nb}$ can also be used as an alternative for low accelerating gradient application. This approach has been taken with great success with the $352 \mathrm{MHz}$ cavities [32] of the Large Electron Positron ring (LEP) at CERN. The possible use of new SC materials with high critical temperature and high upper critical magnetic fields are also investigated. However, the low quality of the SC surfaces 


\section{FUNDAMENTALS OF RADIO FREQUENCY CAVITY \& BEAM DYNAMICS}

produce fairly high losses, which combines with quench problems related to the poor thermal conductivity of SC compounds. A quantity, Residual Resistance Ratio (RRR) or "triple-R", is introduced to specify thermal conductivity and purity of SC material. It is defined as ratio of resistivity of material at room temperature to normal state resistivity of material at $4.2 \mathrm{~K}$.

$$
R R R=\frac{\rho_{300 K}}{\rho_{4.2 K}} .
$$

The approximate relationship between the electron mean free path $(l)$ in $\mathrm{Nb}$ as a function of $R R R$ at $\mathrm{T}=0 \mathrm{~K}$ is [33]:

$$
l(\text { Angstrom })=27 . R R R,
$$

Niobium-Tin $\left(\mathrm{Nb}_{3} \mathrm{Sn}\right)$ [34] is another SC material which appears more favourable since it has a higher $\mathrm{T}_{c}$ of $18.2 \mathrm{~K}$ and a $\mathrm{B}_{s h}$ of $400 \mathrm{mT}$. However, the gradients achieved in $\left(\mathrm{Nb}_{3} \mathrm{Sn}\right)$ coated copper cavities were below $15 \mathrm{MV} / \mathrm{m}$. It may be due to the possible grain boundary effects in the $\mathrm{Nb}_{3} \mathrm{Sn}$ layer.

\subsection{Limiting mechanisms in high gradient SCRF cavity}

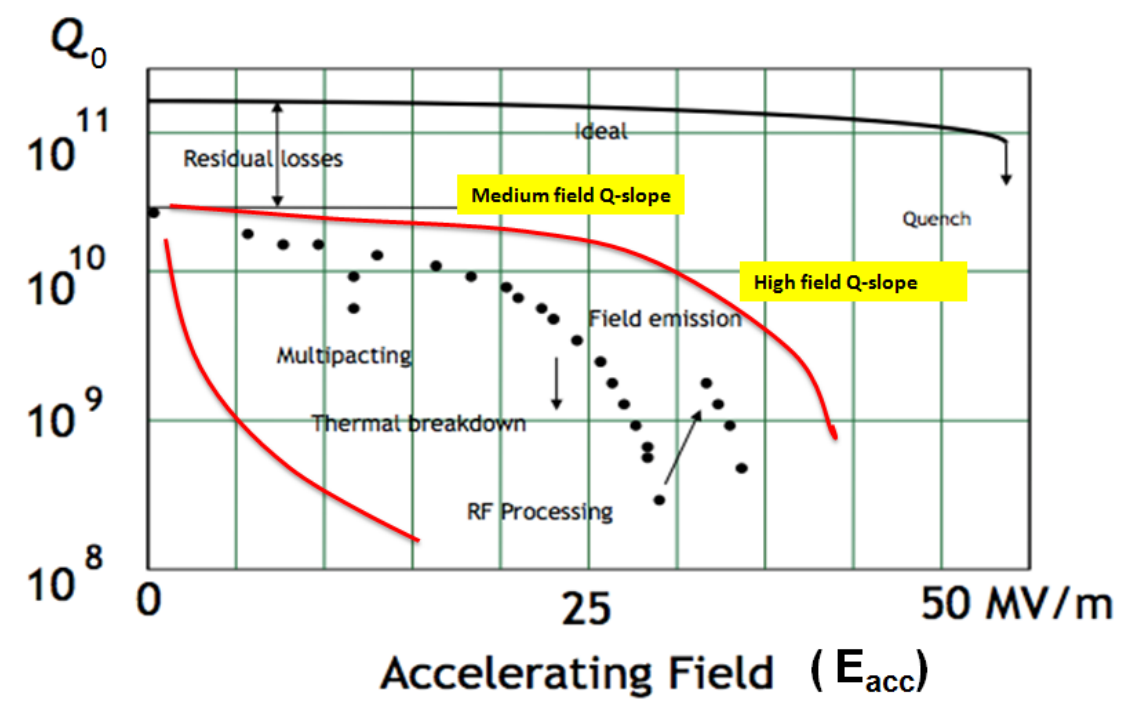

Figure 2.3: $\mathbf{E}_{\text {acc }}-\mathbf{Q}_{\mathbf{0}}$ curve - Impact of multipacting, thermal instability, field emission and residual losses on cavity performance [35].

At present, $\mathrm{Nb}$ cavities are operated at gradients considerably below the theoretical limit of superconductor. The main constraints which limit cavity performances are 
multipacting, FE, thermal instability (or breakdown) and residual losses. Impacts of these limitations on cavity quality factor and accelerating gradients are shown in Figure 2.3. In ideal situation $Q_{0}$ of cavity remains constant but in practical $Q_{0}$ drops gradually (medium field Q-slope) at medium accelerating field and drops very strongly (high field Q-slope) at high accelerating fields. This section provides brief overview of limiting mechanisms and their possible remedies to reach utmost limit of SCRF cavities.

\subsubsection{Multipacting}

Multipacting (multiple impact electron amplification) is observed in RF components which are operated under vacuum such as SCRF cavity and couplers. It is a phenomenon of resonant multiplication of electrons under the influence of RF fields. Basically an electron can be accelerated by the RF field, collides with the wall of the structure and produces secondary electrons. The secondary yield $(\delta)$ counts the number of secondary electrons per incident electron. It is greater than 1 for most metals at an impact energy of the primary electron in the range of 100 to 1000 electron volt $(\mathrm{eV})$. The magnitude of $\delta$ depends on material and surface conditions. It is found that $\delta$ is higher for any uncleaned surface condition. Dirt and condensed gases dramatically enhance $\delta$.

The secondary electrons will be accelerated by the electric component of the RF field and will hit the surface elsewhere. If the time for the trajectory is synchronous with the RF period and if the impact energy is in the range of $\delta>1$, an avalanche of secondary electrons can be created. The synchronous condition depends on details of the geometry and on the magnitude of local electric and magnetic fields. In SCRF cavities, a large number of electrons can be generated which absorb a significant part of the RF energy. These electrons dissipate enough heat to the cavity surface through collisions to trigger a thermal breakdown. Eventually, multipacting limits the achievable accelerating gradient of the cavity. The multipacting depends strongly on the cavity geometry (which affects the resonant trajectories of the electrons) and on its surface condition (which affects the secondary yield). Initially, multipacting was a major performance limitation of SCRF cavity which limited operating accelerating gradient below $10 \mathrm{MV} / \mathrm{m}$. However, it was overcome in SCRF cavities by adopting spherical/elliptical cell shape [36]. In such geometries electrons drift to equatorial region, where electric 


\section{FUNDAMENTALS OF RADIO FREQUENCY CAVITY \& BEAM DYNAMICS}

field is nearly zero thus electron does not have enough impact energy to produce secondary electrons. Multipacting is still not easily determinable. However, it appears at high accelerating gradients. It is usually very weak and easily processed by slowly raising the $\mathrm{RF}$ power ( $\mathrm{RF}$ processing). This kind of multipacting is termed as soft multipacting 24] but in some cases it remains even after RF processing which is called as hard multipacting.

\subsubsection{Field emission}

Field emission (FE) is another well known phenomenon which limits maximum attainable accelerating field in SCRF cavity [24, 37, 38]. When surface electric field is strong, it will lead to electron emission from scratches or particles located on the metal surface through tunneling to form steady current. The FE current increases rapidly as the field is increased. Like the multipacting phenomenon, acceleration of emitted electrons absorbs RF power which would otherwise be available for acceleration of beam. Emitted electrons impact elsewhere on the cavity surface, heating the surface and hence increasing surface resistance. It results in increase in power dissipation of cavity which leads to increase in cryogenic losses. In extreme cases, FE heating of the cavity walls results in quenching of SC states. Fowler and Nordheim [39] developed a theory in which they predicted field emitted current density $\left(J_{F N}\right)$ and total current $\left(I_{F N}\right)$ are given as:

$$
\begin{aligned}
J_{F N} & =\frac{C}{\phi t^{2}(y)} E^{2} \exp \left(-\frac{B \phi^{\frac{2}{3}} v(y)}{E}\right) \\
I_{F N} & =J_{F N} A,
\end{aligned}
$$

where $E$ is the applied electric field, $\phi$ is work function of the metal, $B=8 \pi \sqrt{2 m_{e}} / 3$ he and $C=e^{3} / 8 \pi h$ are constants and $A$ is emitter area. $v(y)$ and $t(y)$ are functions of $y=e^{3} E / 4 \pi \epsilon_{0} \phi^{2}$ which vary slowly with electric field and can be set to 1 in first order approximation.

The experimental signature of $\mathrm{FE}$ is the onset of $\mathrm{X}$-rays and the strong increase of additional losses in the cavity. FE in SCRF cavity can be reduced with careful handling of cavity. Handling procedures which are turned out to be effective in reduction of field emission are: 
- The final cleaning and assembly procedure should be undertaken at stringent dust free conditions.

- Continuous or pulsed operation of the cavity for some time will reduce the strength of $\mathrm{FE}$ (RF conditioning). This operation is more effective if the cavity vacuum is flooded with $\mathrm{He}$ gas in the pressure range of $10^{-5} \mathrm{mbar}$ (He processing).

- High peak power processing (HPPP) [40] is well established way to reduce FE in RF cavity. When using pulsed RF operation with high peak power, very high fields and strong FE loading is observed in the cavity. Some field emitters are destroyed by this operation. Thus, the beginning of FE is shifted to higher cavity fields afterwards.

- High pressure water rinsing (HPWR) of cavity is also very useful to reduce FE.

\subsubsection{Thermal instability}

All previous discussed limitations were related with peak electric field. One typical limitation of the peak magnetic field in a SC cavity is thermal instability. The name quench is commonly used to describe the break down of superconductivity but in RF cavities critical temperature $T_{c}$ is reached due to heating process instead of critical magnetic field. Therefore, thermal instability is appropriate name to describing breakdown of superconductivity in cavity. Thermal breakdown originates at millimeter size that have RF losses substantially higher than the surface resistance of an ideal superconductor. These regions are called defects. These might be either geometrical imperfections such as pits, bumps and hole on cavity surface or external contaminants such as chemical residuals, dust etc.

The breakdown of RF superconductivity is explained by a model of thermal instability [41]. The BCS part of the SC surface resistance has an exponential dependency on temperature. Under steady-state SC conditions the temperature at the inner cavity surface is enhanced by $\Delta T$ as compared to that of the outer surface of cavity (or liquid helium at the outer cavity wall). It is given as:

$$
\Delta T=q\left(\frac{d}{\lambda}+R_{k}\right)
$$




\section{FUNDAMENTALS OF RADIO FREQUENCY CAVITY \& BEAM DYNAMICS}

where $q$ is the heat flux, $d$ is the thickness of the cavity wall, $R_{k}$ is the thermal resistance between the outer cavity wall and the cooling helium (often known as Kapitza resistance) and $\lambda$ is the thermal conductivity of the cavity wall. Existence of the defects cause increase in heat flux due to Joule heating effect which consequently raises the temperature of the inner cavity surface and finally results in an enhanced BCS surface resistance which further produces more heating. There is a critical value of the heat flux at which a self sustained thermal runaway is launched and large parts of the cavity surface will be driven to normal conductivity.

Possibility of thermal instability in cavity can be reduced by:

- avoiding the normal conducting defect by extreme care in preparing and cleaning the cavity surface.

- increase of the thermal conductivity of the SC material.

It is experimentally observed in many cavities, particularly for high frequencies cavities that thermal breakdown occurs well below the critical magnetic field even in the absence of any "defects". This kind of break down is called global thermal instability. It is consequence of quadratic frequency dependence of BCS resistance. It can also appear for low thermal conductivity (low RRR) of cavities. The absence of global thermal instability at low frequency was one of the original important reasons for selecting a frequency near $1 \mathrm{GHz}$ for high accelerating field applications, such as the linear collider.

\subsection{Simulation tools for cavity design}

For most practical RF cavity the geometry is sufficiently complicated that analytical solution for the electromagnetic field distributions and characteristics of eigenmodes supported by cavity does not exist. Development of the cavity demands lot of efforts in terms of both time and money. Thus, it is worthwhile to use simulation tools which enables rapid calculation and optimization of electromagnetic fields of cavities with arbitrary geometry. There exists a number of computer programs designed to solve an eigenvalue problem for accelerating cavities. Some codes are designed to solve it for axially symmetric geometries (two-dimensional, 2-D, codes) such as SLANS [42] and SUPERFISH [43, others can calculate full three dimensional (3-D) problems such as HFSS [44] and CST micro studio [45]. 2-D codes are faster and are therefore preferred 
to optimize cavity shape while 3-D codes need longer runtime to achieve same order of accuracy (mainly because of limitation in generating fine 3-D meshes). 3-D codes are used to simulate fully equipped cavity with power coupler and HOMs coupler. Most of work done on SCRF in this thesis is based on results obtained from SLANS and its family (CLANS, SLANS2, CLANS2 and Tunning).

\subsubsection{Numerical analysis}

Numerical methods for determining electromagnetic fields are based on discretization of the region of interest i.e., subdividing it into discrete elements forming mesh. With sufficient number of intervals, field can be accurately described by calculating their values at discrete points while simple function may be used to describe variation of physical quantity to be determined over the small intervals between mesh points. Several numerical methods have been developed to model the relationship between quantities on adjacent intervals. Finite difference method and finite element method (FEM) [46] are used in many cavity design programs. SLANS uses FEM for calculations and a mesh with bi-quadratic quadrangular elements. It is very important to use appropriate number of mesh points for accurate results.

\subsubsection{Boundary conditions}

A generic computer code requires that the user specifies the cavity geometry, property of materials used (metal or dielectric) and the boundary conditions. Well defined conditions for the fields in boundary plane can be produced by applying boundary conditions in model. The boundary conditions are usually Neumann $\left(E_{\|}=0\right.$, "electric") or Dirichlet $\left(H_{\|}=0\right.$, "magnetic"). It is useful to find symmetry in the problem and utilize it to calculate the full solution by computing only part of the structure if the appropriate boundary conditions are chosen. Figure 2.4 shows the reduction from a 3 -D pill box cavity to a 2-D model by virtue of its cylindrical symmetry, and a further reduction by use of a symmetry plane at the center of the cavity. The boundary condition in effect produces a symmetry condition. 


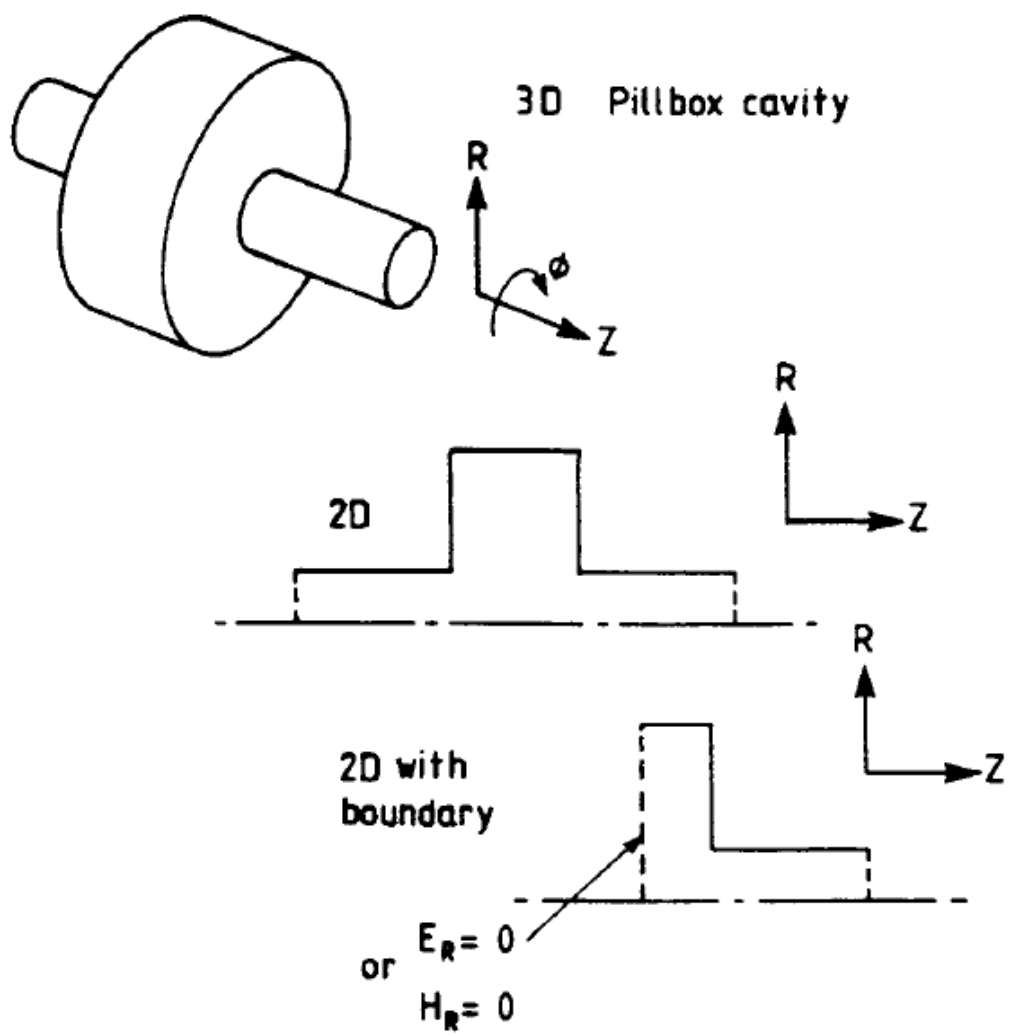

Figure 2.4: Exploiting symmetry in the geometry - Use of symmetry conditions to reduce size of a problem. 


\subsubsection{Description of SLANS}

SLANS calculates the mode frequency and several RF parameters such as the quality factor, stored energy, transit time factor, maximal electric and magnetic fields, acceleration rate etc. The program interface allows plotting of fields along the axis, force lines, and surface fields for a given mode. All fields can be written into output file in ASCII format. Input data for SLANS is presented in a file with extension "geo" describing the boundary of a cavity geometry. The boundary may consist of straight segments and elliptic arcs. If cavity is symmetric, only one half of its geometry may be entered while specifying a boundary condition at the plane of symmetry. These boundary conditions can be either electric wall or magnetic wall. There are more codes belonging to the SLANS family. CLANS solves eigenvalue problem for monopole modes in geometries containing lossy dielectric and ferromagnetic insertions. Programs SLANS2 and CLANS2 calculate azimuthally asymmetric (dipole, quadrupole, etc.) modes in cavities.

\subsubsection{Incorporating practical aspects in simulation}

- Incorporating limiting mechanisms: In high intensity SC accelerator, major cost contribution comes through cryogenic requirements. Thus, to reduce the power dissipation in cavity and hence cryogenic losses, cavities are operated in high Q regime. Accelerating gradients are chosen in medium- Q slope region (Figure 2.3) which further puts constraints on the peak surface fields.

- Operation at low temperature: Simulation is performed to optimize the cavity shape at operating temperature (typically $2 \mathrm{~K}$ ). However, fabrication dimensions of cavity are different from designed dimensions. It includes thermal expansion of material during transition of cold temperature $(2 \mathrm{~K})$ to room temperature $(300 \mathrm{~K})$ and material removal during surface processing of cavity such as electro polishing (EP) and buffer chemical polishing (BCP).

In next sections the basics of beam physics which are required to address beam motion and fundamentals of lattice design are introduced. Concept of design of high intensity ion linac and high intensity effects such as space charge effects are also discussed. 


\section{FUNDAMENTALS OF RADIO FREQUENCY CAVITY \& BEAM DYNAMICS}

\subsection{Beam dynamics}

The force $(F)$ experienced by a particle with charge $q$ traveling in presence of electric and magnetic fields is governed by Lorentz force:

$$
\vec{F}=\frac{d \vec{P}}{d t}=q(\vec{E}+\vec{v} \times \vec{B}) ;
$$

where $\vec{P}$ and $\vec{v}$ are momentum and velocity of particle respectively. In a high energy accelerator magnetic fields are mainly used for bending, focusing and correction of the beam while electric fields are commonly used for acceleration. RF cavities are utilized to provide electric fields. Dipole magnets are used for bending and correcting beam trajectories while quadrupole and solenoids are used for focusing the beam along the axis.

\subsubsection{Coordinate system}

Cartesian coordinates are used globally in describing the particle position and motion throughout the beam line. Location of RF cavities, magnets and other components in accelerator are defined along the reference or nominal trajectory by ' $s$ '. Transverse coordinates of charge particles with respect to reference particle in horizontal and vertical directions are represented by $x$ and $y$ respectively. In a linac, reference trajectory is a straight line defined by symmetry axis of beam line elements. In circular accelerator reference trajectory is not straight and therefore curvature is taken into account using Frenet's frame.

Time derivative of particle positions give velocities of particle in respective directions $\left(v_{x}, v_{y}, v_{z}\right)$. Derivative of particle position with respect to ' $s$ ' is given as :

$$
x^{\prime}=\frac{d x}{d s} ; \quad y^{\prime}=\frac{d y}{d s}
$$

where prime represents derivative with respect to $s$. In general particle's longitudinal velocity in laboratory frame is much higher than transverse velocities.

$$
v_{z} \gg v_{x} \text { and } v_{z} \gg v_{y}
$$

Therefore,

$$
v=\sqrt{v_{z}^{2}+v_{x}^{2}+v_{y}^{2}} \approx v_{z}
$$


taking into account paraxial approximation, equation 2.66 can be written as following:

$$
\begin{aligned}
& \frac{d x}{d s}=\frac{\dot{x}}{\dot{s}}=\tan \theta_{x} \approx \theta_{x} \\
& \frac{d y}{d s}=\frac{\dot{y}}{\dot{s}}=\tan \theta_{y} \approx \theta_{y}
\end{aligned}
$$

In above equations $\theta_{x}$ is angle between $v_{z}$ and $v_{x}$. Similarly $\theta_{y}$ is angle between the $v_{z}$ and $v_{y}$. Thus, $x^{\prime}$ and $y^{\prime}$ provide information about convergence and divergence of beam in transverse plane.

Longitudinal direction is defined in direction of motion of charge particle. Beam coordinates in longitudinal plane are represented in terms of time and energy as canonical coordinates. If $t_{0}$ and $W_{0}$ are time and energy coordinates of reference particle respectively, all other particles in bunch can be expressed relative to reference particles as following:

$$
\begin{aligned}
\Delta t & =t-t_{0} \\
\Delta z & =\beta . c . t-\beta_{0} . c . t_{0} \\
\Delta z & =z-z_{0} \\
\Delta \phi & =\phi-\phi_{s} \quad \forall \phi=2 \pi t
\end{aligned}
$$

and

$$
\begin{aligned}
\Delta W & =W-W_{0} \\
\delta & =\frac{P-P_{0}}{P_{0}} \\
\Delta z^{\prime} & =\frac{v-v_{0}}{v_{0}} ;
\end{aligned}
$$

where $z_{0}, P_{0}, v_{0}$ and $\phi_{s}$ are longitudinal position, momentum, velocity and synchronous phase of reference particle respectively.

\subsubsection{Longitudinal synchrotron motion}

In order to achieve continuous acceleration in an accelerator, particle must be synchronous with the RF wave. It means that particle must be injected in accelerator on a well defined phase with respect to the sinusoidal field and it has to maintain this phase during the acceleration process. However, beams are made of large number of particles with certain spread in phase and in energy. If the injection phase correspond to the 


\section{FUNDAMENTALS OF RADIO FREQUENCY CAVITY \& BEAM DYNAMICS}

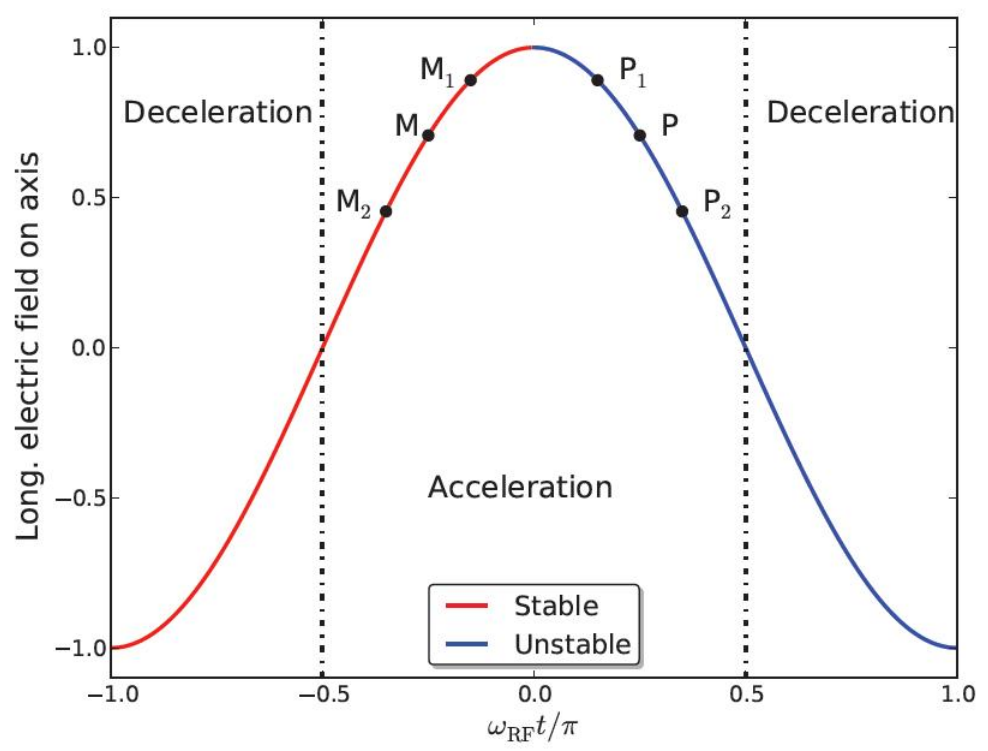

Figure 2.5: Longitudinal phase stability description of particles in a bunch.

crest of the wave $\left(\phi=0^{\circ}\right)$ for maximum acceleration, particles having slightly higher or lower phase gain less energy and hence they will slowly loss synchronicity until they are lost completely. Thus, two possibilities for beam injecting phase are left which could be either side of crest between $\phi=-\pi / 2$ to $\phi=\pi / 2$ as shown in Figure 2.5 .

Taking the negative phase into account, consider three particles $\mathrm{M}_{1}, \mathrm{M}$ and $\mathrm{M}_{2}$ where $\mathrm{M}$ is synchronous particle. $\mathrm{M}_{1}$ sees higher field and hence gains more energy than $\mathrm{M}$ while $\mathrm{M}_{2}$ sees lower field and hence gains less energy than M. In next RF cycle, $\mathrm{M}_{1}$ reaches early than $\mathrm{M}$ and thus sees lower field but $\mathrm{M}_{2}$ reaches late due to lower energy, seeing higher field. This process results in oscillation of $\mathrm{M}_{1}$ and $\mathrm{M}_{2}$ around the synchronous particle $\mathrm{M}$ which always arrives in correct phase and receives same energy. These oscillations are called "synchrotron oscillations" and these oscillations are represented by elliptical motions of each particle in longitudinal plane of phase and energy difference $(\Delta \phi$ and $\Delta W)$ with respect to synchronous particle. Resulting longitudinal motion is stable. This principle of phase stability is valid for ion beam which is still non relativistic. The schematic of longitudinal motion of ion beam which shows relationship between the synchronous phase in an accelerating sinusoidal field and the longitudinal phase plane is shown in Figure 2.6. All the stable phase trajectories are closed and are confined in separatrix. 


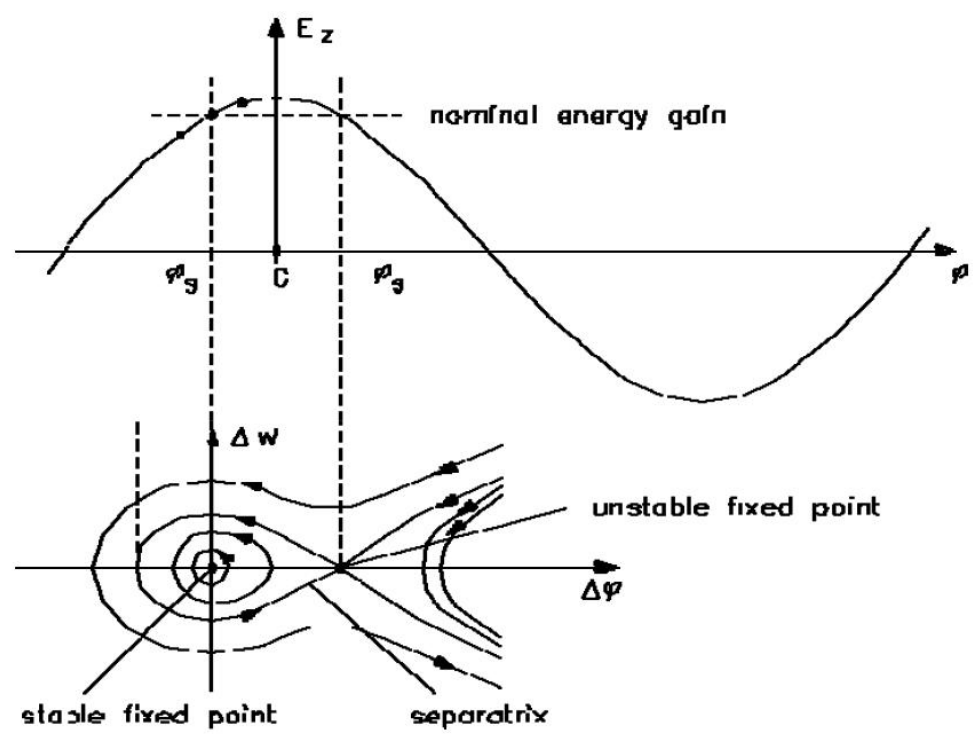

Figure 2.6: Longitudinal motion of an ion beam.

For the small phase amplitude oscillations, equation of longitudinal motion can be written as:

$$
\begin{aligned}
\frac{d(\Delta \phi)}{d s} & =-2 \pi \cdot \frac{\Delta W}{(\beta \gamma)^{3} \cdot m c^{2} \cdot \lambda} \\
\frac{d(\Delta W)}{d s} & =q \cdot E_{0} T_{s} \cdot \sin \phi_{s} \cdot \Delta \phi ;
\end{aligned}
$$

where $s$ is abscissa of the beam in the linac path, $E_{0}$ is average electric field along the axis, $T_{s}$ and $\phi_{s}$ are transit time factor and phase of synchronous particle respectively, and $\lambda$ is RF wavelength. Equation 2.77) can be decoupled to give second order differential equation of phase evolution [47]:

$$
\frac{d^{2}(\Delta \phi)}{d s^{2}}+\frac{2}{\varsigma} \cdot \frac{d(\Delta \phi)}{d s}+k_{z}^{2} \cdot \Delta \phi=0
$$

where

$$
k_{z}^{2}=\frac{2 \pi q \cdot E_{0} T_{s} \cdot \sin \left(-\phi_{s}\right)}{(\beta \gamma)^{3} \cdot m c^{2} \cdot \lambda}
$$

$k_{z}$ is phase advance per meter of the beam core. In a periodic lattice of period $L$, $\sigma_{z}=k_{z} L$ is longitudinal core phase advance per lattice. The damping length ( $\varsigma$ ) of the core oscillations is given as:

$$
\varsigma=\frac{2}{3} \cdot \frac{\beta \gamma}{d(\beta \gamma) / d s}
$$




\section{FUNDAMENTALS OF RADIO FREQUENCY CAVITY \& BEAM DYNAMICS}

It is interesting to observe that $k_{z}$ depends on beam velocity. When beam becomes relativistic, phase advance decreases rapidly. At the limit of $(\beta \gamma)^{3} \gg 1$ oscillation will stop and beam is practically frozen in phase and energy with respect to the synchronous particle.

\subsubsection{Transverse betatron motion}

In an accelerator, additional fields other than accelerating fields are also required to focus the beam along the axis. Principal factors which results in transverse displacement of beam are:

- Random spread of transverse thermal velocities of particles.

- Transverse kick by accelerating structure.

- Repulsion among the particles in the beam.

In an accelerator transverse forces acting on beam are mainly due to focusing magnet. In ideal situation, focusing magnets do not affect the trajectory of a synchronous particle. Other particles in bunch which are deviated from reference trajectory perform oscillation along the reference trajectory. These oscillations of particles around reference trajectory are called "betatron oscillations". Assuming no dispersion and small amplitude betatron oscillation around reference trajectory, the motion of particles are governed by second order differential equation, known as Hill's equation.

$$
\begin{aligned}
& x^{\prime \prime}+K_{x}(s) x(s)=0 \\
& y^{\prime \prime}+K_{y}(s) y(s)=0
\end{aligned}
$$

where

$$
K_{x}=\frac{1}{\rho^{2}}-\frac{\partial B_{y}}{\partial x} \cdot \frac{1}{B \rho} ; \quad K_{y}=\frac{\partial B_{x}}{\partial y} \cdot \frac{1}{B \rho},
$$

where $\rho$ is radius of curvature, $B \rho=P / e$ is beam rigidity also called magnetic rigidity, $P$ is momentum of reference particle and $\frac{\partial B_{y}}{\partial x}, \frac{\partial B_{x}}{\partial y}$ are quadrupole field gradients. It should be noted that additional focusing term $1 / \rho^{2}$ in $x$ plane vanishes for linac due to absence of bending magnets.

In linac, focusing elements are arranged in a lattice which is either periodic or period is changed adiabatically to allow for increasing period length as beam is accelerated. 
Thus, Hill's equation remains valid. The solutions of Hill's equations for ideal periodic lattice are:

$$
\begin{aligned}
x(s) & =\sqrt{2 J \beta_{t}(s)} \cos \left(\psi(s)+\phi_{a}\right) \\
x^{\prime}(s) & =\sqrt{\frac{2 J}{\beta_{t}(s)}}\left[\sin \left(\psi(s)+\phi_{a}\right)+\alpha(s) \cos \left(\psi(s)+\phi_{a}\right)\right] ;
\end{aligned}
$$

where $J$ and $\phi_{a}$ are action angle invariants of motion, $\beta_{t}(s)$ is betatron function, $\alpha(\mathrm{s})$ $=-\beta_{t}^{\prime}(\mathrm{s}) / 2$ and $\psi(\mathrm{s})$ is the phase advance given by:

$$
\psi\left(s_{1} \rightarrow s_{2}\right)=\int_{s_{1}}^{s_{2}} \frac{1}{\beta_{t}(s)} d s .
$$

The action angle $J$ can be expressed in term of $x$ and $x^{\prime}$ to yield the Courant-Snyder invariant [48].

$$
2 J=\gamma_{t} x^{2}+2 \alpha x x^{\prime}+\beta_{t} x^{2}=\epsilon,
$$

where

$$
\gamma_{t}=\frac{1+\alpha^{2}(s)}{\beta_{t}(s)},
$$

$\alpha, \beta_{t}$ and $\gamma_{t}$ are collectively called Courant Snyder parameters and commonly known as Twiss parameters. In phase space $\left(x, x^{\prime}\right)$ particle trajectory forms an ellipse, and area of this ellipse is given by:

$$
\text { Area }=\pi \epsilon
$$

where " $\epsilon$ " is called emittance. All particles in bunch trace different contours (ellipse) in phase space. Area bounded by largest ellipse which occupies all particles trajectories in phase space gives beam emittance. Figure 2.7 shows different contours that correspond to given fraction of the total beam distribution. The area within a particular contour is emittance for that particular beam fraction. For each fraction we get a different value of the beam emittance. Thus, beam emittance is typically referred as the $90 \%$ or $95 \%$ fractional emittance.

In the absence of non linear forces and weak interaction among particles in bunch, beam follows Liouville's theorem which results in conservation of phase space area and hence emittance.

In presence of acceleration, transverse emittance starts to shrink because acceleration changes longitudinal momentum more rapidly than transverse momentum which reduces slope of beam in transverse directions $\left(x^{\prime}, y^{\prime}\right)$. This shrinkage of transverse 


\section{FUNDAMENTALS OF RADIO FREQUENCY CAVITY \& BEAM DYNAMICS}

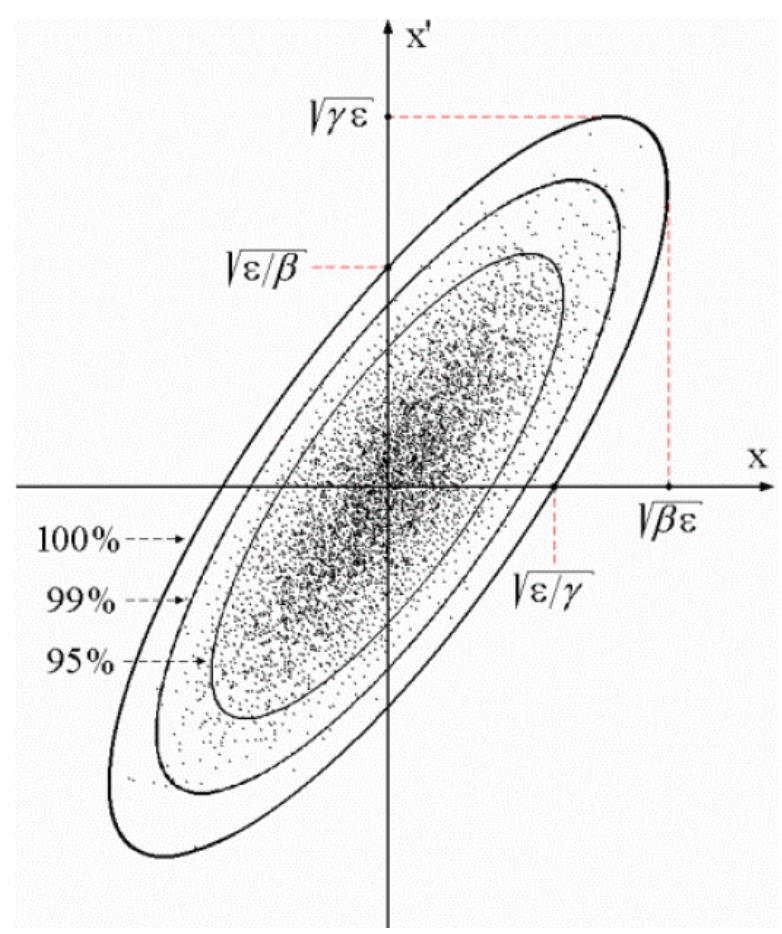

Figure 2.7: Courant-Snyder ellipse in $\left(x, x^{\prime}\right)$ phase space.

beam ellipse is called adiabatic damping. Thus to compare beam quality at different locations in accelerator, normalized emittance $\left(\epsilon_{N}\right)$ is introduced which is given as:

$$
\epsilon_{N}=\beta \gamma \epsilon,
$$

where $\beta$ and $\gamma$ are relativistic parameters. $\beta$ and $\gamma$ also increase with acceleration thus normalized emittance remains invariant in accelerator and can be used as a figure of merit of beam.

In high intensity beam, interaction among particles are not negligible. Presence of non-linear forces results in filamentation which leads to decrease in beam core density. Particles in outermost part of core start to spread in phase space. Figure 2.8 shows a representation of beam phase space area in presence of filamentation. Thus, usual definition of beam emittance as the area bounded by ellipse suffers in presence of distorted phase space. A statistical definition of beam emittance is introduced for such scenario based on second order momenta of particle distribution function. If for a system of $N$ particles the average value of $X$ is $\langle X\rangle=\frac{1}{N} \sum_{i=1}^{N} X_{i}$ where $i$ is number 


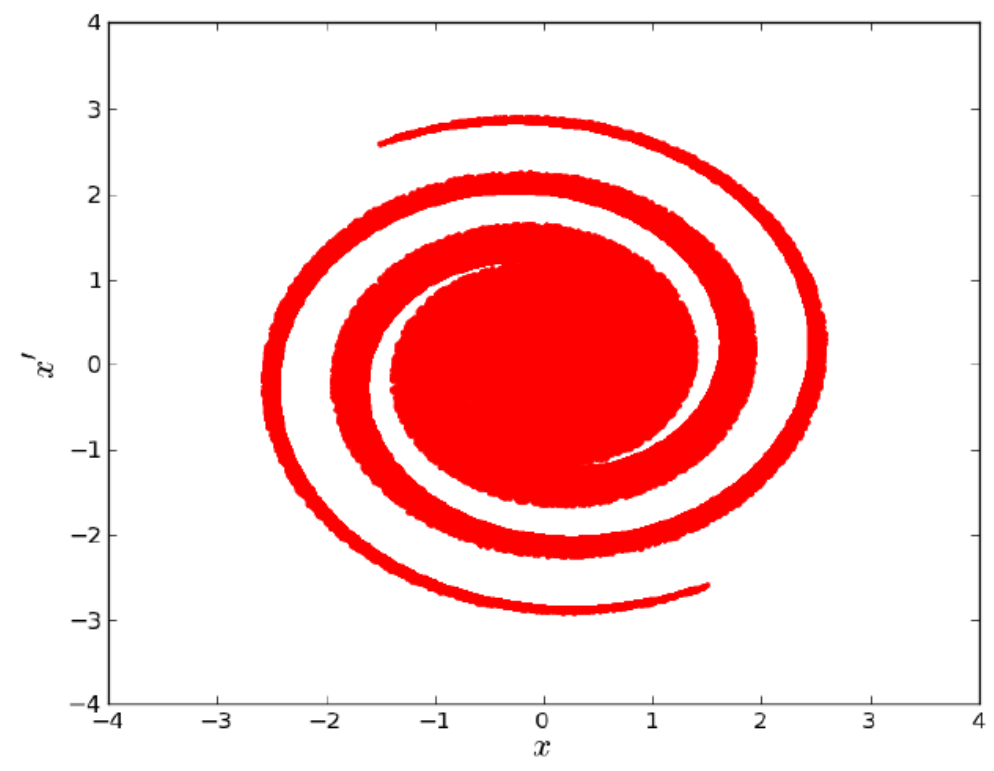

Figure 2.8: A typical representation of beam phase space area in presence of non linear forces.

of particles. For the $w=x, y$ or $\Delta z$ :

$$
\begin{aligned}
\text { Beam RMS size: } \widetilde{w} & =\sqrt{\left\langle(w-\langle w\rangle)^{2}\right\rangle} \\
\text { Beam RMS divergence: } \widetilde{w^{\prime}} & =\sqrt{\left\langle\left(w^{\prime}-\left\langle w^{\prime}\right\rangle\right)^{2}\right\rangle} \\
\widetilde{w w^{\prime}} & =\sqrt{\left\langle(w-\langle w\rangle) \cdot\left(w^{\prime}-\left\langle w^{\prime}\right\rangle\right)\right\rangle}
\end{aligned}
$$

Root Mean Square (RMS) emittance can be defined as:

$$
\tilde{\epsilon}_{w, w^{\prime}}=\left(\widetilde{w}^{2} \cdot{\widetilde{w^{\prime}}}^{2}-\left({\widetilde{w w^{\prime}}}^{2}\right)\right)^{1 / 2}
$$

Considering that beam is at center $\left(\langle w\rangle=0\right.$ and $\left.\left\langle w^{\prime}\right\rangle=0\right)$, normalized RMS emittance in all three planes can be obtained as:

In $\left(\mathrm{x}, \mathrm{x}^{\prime}\right)$ plane:

$$
\widetilde{\epsilon}_{x, n}=\beta \gamma\left(\langle x\rangle^{2} \cdot\left\langle x^{\prime}\right\rangle^{2}-\left\langle x x^{\prime}\right\rangle^{2}\right)^{1 / 2}
$$




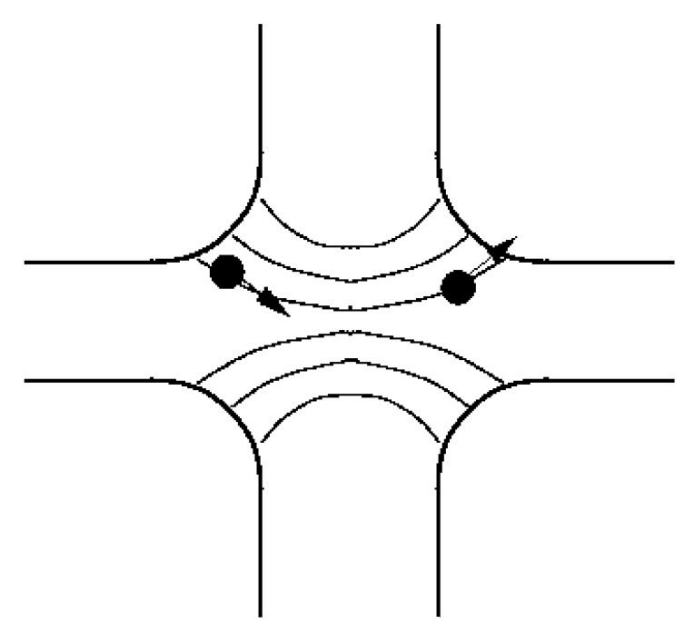

(a)

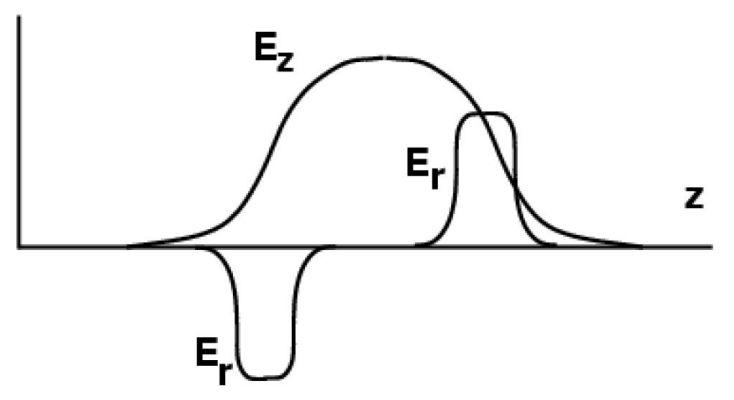

(b)

Figure 2.9: (a) Distribution of field lines across RF gap and (b) radial and axial field distribution in a RF gap.

In $\left(y, y^{\prime}\right)$ plane:

$$
\widetilde{\epsilon}_{y, n}=\beta \gamma\left(\langle y\rangle^{2} \cdot\left\langle y^{\prime}\right\rangle^{2}-\left\langle y y^{\prime}\right\rangle^{2}\right)^{1 / 2}
$$

In $\left(\mathrm{z}, \mathrm{z}^{\prime}\right)$ plane:

$$
\widetilde{\epsilon}_{z, n}=\beta^{2} \gamma^{3}\left(\langle\Delta z\rangle^{2} \cdot\left\langle\Delta z^{\prime}\right\rangle^{2}-\left\langle\Delta z \Delta z^{\prime}\right\rangle^{2}\right)^{1 / 2}
$$

The beam Twiss parameters can also be deduced from beam RMS dimensions and emittance as follows:

$$
\widetilde{\beta}_{t w}=\frac{\widetilde{w}^{2}}{\widetilde{\epsilon}_{w}}, \quad \widetilde{\gamma}_{t w}=\frac{\widetilde{w}^{\prime}}{\widetilde{\epsilon}_{w}}, \quad \widetilde{\alpha}_{w}=-\frac{\left\langle(w-\langle w\rangle) \cdot\left(w^{\prime}-\left\langle w^{\prime}\right\rangle\right)\right\rangle}{\widetilde{\epsilon}_{w}} ;
$$

\subsubsection{RF defocusing by accelerating structure}

RF cavity not only provides acceleration to charge particles but it also give transverse kick to particles which results in radial offset of particle. Defocusing mechanism in cavity can be explained with the help of Figures $2.9 \mathrm{a}$ and $2.9 \mathrm{~b}$.

It can be seen from Figure 2.9a that when an offset particle enters in the RF gap, it sees additional radial electric field which exists at ends of the gaps (Figure 2.9b). Radial field at the entrance of gap directs the particle toward the axis but radial field at the 
exit of gap directs it away from the axis. As particle has already been accelerated so it spends less time at exit of RF gap than entrance which means deflection of particle trajectory turns out to be greater at entrance. It results in net focusing of particle toward the axis. It all happens in ideal case when fields are not changing with time. In reality, field in standing wave structure varies with time. As we discussed earlier that for stable longitudinal motion, bunch is placed at negative phase. Thus, field is increasing as the bunch passes across the gap. It means that particles in bunch near the reference particle see lower field at the entrance and relatively higher field at exit of gap which results in defocusing of bunch. Radial force $\left(F_{r}\right)$ [49] on offset particle due to time varying electric field in accelerating structure can be expressed as:

$$
F_{r} \simeq-q E_{0} \cdot \frac{\omega_{R F} r}{2 \beta c \gamma^{2}} \sin \phi_{s}
$$

where $r$ is offset of particle from axis and $\omega_{R F}$ is RF angular frequency.

\subsection{High intensity effects in linear accelerator}

When the intensity of the beam increases, one is confronted with two distinct effects in a linac:

- Repulsion of particles being close together in bunch i.e., known as space charge effect.

- Excitation of fields on the wall of cavity. It results in image effects for non relativistic beam and wake field and beam break up (BBU) for relativistic beam.

\subsubsection{Space charge effect}

The importance of space charge effects on beam optics decreases as $\propto\left(\beta^{-2} \gamma^{-3}\right)$. Thus, it is less important for electron but one has to take it into account for intense ion beam for effective lattice design. As we know that transverse trajectories are ellipse in phase space under the influence of linear forces. In transverse planes, the external focusing forces are essential linear while in longitudinal plane the linear region is limited to the neighbourhood of synchronous phase. Charge density distribution in the beam is usually non-uniform which results in non-linear space charge forces. It is possible to match the beam with transport line when space charge forces are linear and hence these 


\section{FUNDAMENTALS OF RADIO FREQUENCY CAVITY \& BEAM DYNAMICS}

forces are first linearized. It can be performed by replacing real beam with equivalent beam of same RMS size but with uniform charge density distribution. It is demonstrated [50] that evolution of RMS beam size essentially depends only on linear part of space charge forces. It is valid for beam of any charge distributions, provided they are of the ellipsoidal type i.e., iso-density curves are concentric ellipse. Hence, the RMS beam size and and RMS emittance can be matched. All beams having the same intensity, and the same RMS values (when RMS emittance is not growing) behave in the same way. Therefore, once RMS values of a real beam is known it can be replaced by an 'equivalent' one having the same RMS sizes but with a uniform charge density distribution in the accelerator design process. The importance of non-linear effects is then checked by using beam simulation program. Single particle tune in high current linac obeys [51]:

$$
\sigma_{\text {core }} \leq \sigma_{p} \leq \sigma_{0}
$$

where $\sigma_{\text {core }}, \sigma_{p}$ and $\sigma_{0}$ are phase advance of beam core, phase advance of particle outside the core and phase advance of particle in absence of any space charge (zero current) respectively. The strength of space charge forces can be measured in terms of parameter called tune depression $(\eta)$.

$$
\eta=\frac{\sigma}{\sigma_{0}}<1
$$

Since space charge forces are defocusing in nature, beam core phase advance gets reduced than phase advance without space charge and results in tune depression less than 1. Strong tune depression in high current linac results in large single particle tune spread (equation 2.100) which might lead to parametric resonances [51]. On the other hand higher value of tune depression means lower influence of space charge force.

Linear approximation of space charge force is essential from lattice design point of view but in fact it is only an approximation. Beam emittance growth is observed in high intensity high energy linear accelerator even if the equivalent beam is RMS matched to the accelerator acceptance. Typical reasons of emittance growth in high intensity linac are:

- Mismatch of beam: It is observed that envelope oscillations ('breathing' in phase or phase opposition), are damped in a non-linear regime, but the final 
beam radius is always larger then the original matched one. If the beam radius oscillates between $\tilde{x}_{\max }$ and $\tilde{x}_{\min }$, the final radius will be

$$
\tilde{x}_{f}=\sqrt{\frac{\tilde{x}_{\max }^{2}+\tilde{x}_{\min }^{2}}{2}}
$$

leading to a large emittance. However, this effect is small for small mismatch.

- Halo formation: The existence of beam halo is an important characteristic of high-intensity beams. A fraction of beam particles acquire enough transverse energy within beam which results in large tail known as beam halo. Halo amplitude depends not only on the mismatch but intensity of non-linear forces also. Halo formation is result of severals effects such as misalignment of beam line elements, misadjusting of focusing gradients and other non-adiabatic changes in lattice. Distribution of particles in outer core is quantified using parameter called beam halo parameter $(\mathrm{H})$ [52]. Halo parameter is invariant under conservative force and its growth corresponds to non-conservative forces, as with emittances.

Beam halo results in emittance growth and beam losses at high energy section in linac. It might lead to radioactivity. In order to reduce beam losses, physical aperture of beam line elements should be large. Use of SC cavities allows one to increase aperture without sacrificing much in term of RF performance. In longitudinal direction, use of short bunches and opting synchronous phases in linear regime especially at low energy reduces the longitudinal beam losses.

- Coupling between longitudinal and transverse plane: Coupling between the longitudinal and transverse plane via space charge forces can cause emittance growth if the bunches have different longitudinal and transverse temperatures. It should be noted that here temperature is related with beam rms size and beam normalized emittance.

$$
T_{x}=\frac{\epsilon_{x r m s}^{2}}{x_{r m s}^{2}}
$$

where $T_{x}$ is horizontal temperature. If temperature is high in one plane, coupling of phase planes will results in heat (kinetic energy of particles) flow from one plane to another till temperatures are equalized in each of planes in the beam 


\section{FUNDAMENTALS OF RADIO FREQUENCY CAVITY \& BEAM DYNAMICS}

rest frame. It is called equipartitioning or thermalization. It occurs in presence of high space charge densities. The condition of equipartitioning is given as:

$$
\frac{\epsilon_{x r m s}^{2}}{x_{r m s}^{2}}=\frac{\epsilon_{y r m s}^{2}}{y_{r m s}^{2}}=\frac{\epsilon_{z r m s}^{2}}{\gamma^{2} z_{r m s}^{2}} .
$$

\subsubsection{Image charge effect}

In a real accelerator beam is surrounded by RF cavities, vacuum tube and magnets. Beam self field induces the surface charge or current into this environment which acts back on the beam and results in transverse defocussing of beam. However, this effect is very weak and can be usually neglected due to large transverse aperture of beam line elements with respect to beam size. The only place where such effects are sometimes considered is in RFQs having a small aperture and being used for very low particle velocities. A highly intense and misaligned beam can also trigger this effect.

\subsection{Beam dynamics codes for lattice design}

Beam optics is designed and optimized using GENLINWIN [53] and TRACEWIN [54]. A user defined beam line can be constructed from TRACEWIN library of beam line elements which include quadrupole magnets, solenoid and standard accelerating cavities etc. There is a possibility to implement field corresponding to these elements using external field maps. GENLINWIN is used to tune longitudinal beam dynamics. It helps to set appropriate field amplitudes and RF phases in cavities. TRACEWIN is used to tune transverse beam dynamics and helps to set field gradients in quadrupoles and solenoids. TRACEWIN can generate as well as read input file for beam distribution. Beam input parameters, such as the emittance, Twiss's parameters and beam charge density, can be specified at the start of the beam line and propagated through the beam line model. The evolution of the beam parameters such as emittance, Twiss parameters, energy, beam size etc. can be calculated and plotted at the exit of each element using standard principle of beam dynamics. It is capable to perform matching between two segmented sections in lattice and allows to match the beam to a channel. It permits fast beam envelope computation. It also consists of module called PARTRAN which is able to perform multi-particle tracking with two dimensional or three dimensional space charge computation. It enhances the capability of TRACEWIN and allows one to study the impact of high intensity effects (discussed earlier) on beam dynamics. 
It is always good to cross-check results with other independent beam dynamics code. Thus, TRACEWIN results are benchmarked with TRACK. TRACK [55] is designed at Argonne national laboratory(ANL) in USA. It is originally developed for design and study of the beam dynamics at rare isotope accelerator (RIA) facility. 
2. FUNDAMENTALS OF RADIO FREQUENCY CAVITY \& BEAM DYNAMICS 


\section{3 \\ Design of SCRF cavity for the Project-X linac}

This chapter describes cavity design for the Project-X linac. Since Project-X linac has evolved over recent years from pulsed version to continuous wave (CW) version, so cavities are designed for both of these versions. In the pulsed (earlier) version of the linac, there already exists a 11-cell, $1.3 \mathrm{GHz}$ design of cavity [20, 56, 57]. HOMs study of the cavity is performed and asymmetrical design of the end cell is proposed. Further, an alternate version of the cavity design based on 9-cell is also proposed.

In latest $(\mathrm{CW})$ version of the linac, 5-cell, $650 \mathrm{MHz}$ cavities are designed for the intermediate and high energy sections corresponding to $\beta_{G}=0.61$ and $\beta_{G}=0.90$. Shapes of $\beta_{G}=0.61$ and $\beta_{G}=0.90$ cavities are optimized to achieve maximum acceleration and minimum power dissipation to reduce cryogenic losses. Field enhancement factors are minimized to increase the accelerating gradient while $R / Q$ and geometrical factor $(G)$ for operating mode are maximized to reduce the power dissipation to the wall of cavity. Optimization of shape and calculation of RF parameters such as field enhancement factors, quality factor, shunt impedance etc. of cavity are performed using two-dimensional cavity design code SLANS. HOMs spectrum is studied for both of these cavities. The cavities are designed in such a way that there are no significantly trapped HOMs. A systematic study is performed to understand the effects of excitation of resonance of HOMs on beam quality and resultant power dissipation. Analysis of HOMs provides better understanding of the requirements of HOM damper for these cavities. 


\section{DESIGN OF SCRF CAVITY FOR THE PROJECT-X LINAC}

Since this chapter mainly discusses the cavity design so in the next section, a general description of designing cavity geometry is discussed.

\subsection{Design of cavity geometry}

Design of cavity geometry means searching for an optimal cell shape which accomplishes all operational requirements. Over the period of times a simple cylindrical shaped cavity is emerged into elliptical shaped cavity. Important features of elliptical shape are:

- In cylindrical shaped SCRF cavity multipacting and field emission had been the major problems for many years. They limited maximum achievable gradient in SCRF cavity. Development of elliptical shaped cavity resulted in overcome with these problems.

- SCRF cavities are treated with chemicals to achieve smooth and clean inner surface. The continuous curvature and tilting of the elliptical shaped cavity allow good access for surface treatments resulting in a reduction of chemical residues, which often enhance the losses and the electron emission.

- The tilt of the elliptical cavity also increases the stiffness against mechanical deformations due to pressure applied by helium vapours during operation.

- The elliptical geometry yields reduced electric field and magnetic field enhancement factor. It results in enhancement in maximum achievable accelerating gradient in SCRF cavity.

A multi-cell elliptical cavity (Figure 2.1) is composed of two kind of cells: "inner cell" or regular cell and "end cell". The end cells are different from the inner cells as they are used to connect cavity with beam pipe at both ends so that beam can pass through cavity. Thus, the end cells need to be designed separately from inner cell to maintain operating frequency of cavity. The optimum design of the inner and end cells is a consequence of the series of trade-off between different cavity parameters, ranging from RF to mechanics. Few of these parameters get frozen on the basis of the constraints arising from the fundamental and practical aspects, which are described below. 


\subsubsection{Fundamental aspects}

Fundamental aspects comprise of operational requirements and beam specifications. Two characteristic cavity parameters which are determined on the basis of these requirements are operating frequency and number of cells in a multi-cell cavity.

Choice of operating frequency: The choice of frequency is the one of critical factor for the cavity design. For instance, cavities are operated at low frequency in low energy section of ion linac. Lower frequency allows one to use large aperture. Thus, it provides large transverse acceptance that helps to reduce beam losses which are more prominent particularly at low energy in high intensity linac (where space charge effects might be significant). Large transverse aperture also reduces the wakefield effects. It also results in extraction of HOMs more efficiently and hence results in significant reduction of HOMs power deposited in the cavity that helps to increase the threshold current for multi-bunch instabilities. As discussed in previous chapter, that surface resistance $\left(R_{s}\right)$ of superconductor is proportional to square of frequency for $\mathrm{R}_{B C S} \gg$ $R_{0}$ and becomes independent of frequency for $\mathrm{R}_{B C S} \ll R_{0}$. In SCRF Nb cavities, at $\mathrm{T}=2 \mathrm{~K}$ the $\mathrm{BCS}$ term dominates above $3 \mathrm{GHz}$ and for a given length of cavity RF losses increase linearly with frequency, whereas below $300 \mathrm{MHz} R_{0}$ dominates and RF losses are inversely proportional to frequency. To minimize power dissipation to the cavity wall one generally selects frequency in operating range between $300 \mathrm{MHz}$ to $3 \mathrm{GHz}$ so that $R_{s} \approx R_{0}$. However, lower frequency and consequently larger aperture cavities also increase the fabrication cost. Higher frequency cavities are thus smaller and hence cheaper. Cavities are usually operated at high frequency in the high energy section of accelerator. The exact choice of the operating frequency is also influenced by other accelerator components such as availability of klystron, power coupler etc.

Number of cells in a cavity: Multi-cell cavity is a good choice for accelerator in many ways but limited velocity acceptance for non-relativistic beam, possibility of existence of trapped modes and difficulties in extraction of HOMs power are main constraints which limits increasing the number of cells. Choice of number of cells in cavity is made on the basis of following.

- Energy gain per cavity: For big aperture of cavity, the distribution of axial electric field $E_{z}$ for $\pi$-mode can be approximated by sinusoidal field distribution, 


\section{DESIGN OF SCRF CAVITY FOR THE PROJECT-X LINAC}

given as:

$$
\begin{aligned}
E_{z}= & G \cdot \cos \left(k z / \beta_{G}\right) \quad \text { if } N \text { is odd, } \\
= & G \cdot \sin \left(k z / \beta_{G}\right) \quad \text { if } N \text { is even, } \\
& -L_{\text {cav }} / 2<z<L_{\text {cav }} / 2 .
\end{aligned}
$$

Where $G$ is the field amplitude, $L_{\text {cav }}$ is length of the cavity and $N$ is number of cells in a cavity. For any arbitrary particle which is moving with relative velocity $\beta$ the energy gain per cavity $\Delta u(N)$ is obtained by integrating equation (3.1) and together with the phase term, $\exp (i \omega t)=\exp (i k z / \beta)$, over the cavity length. It is given as:

$$
\begin{aligned}
\Delta u(N) & =\left|\frac{G \lambda \beta_{G}\left(\beta / \beta_{G}\right)^{2}}{\pi\left[\left(\beta / \beta_{G}\right)^{2}-1\right]} \cos \left(\frac{\pi N \beta_{G}}{2 \beta}\right)\right|, \quad \text { if } N \text { is odd; } \\
\Delta u(N) & =\left|\frac{G \lambda \beta_{G}\left(\beta / \beta_{G}\right)^{2}}{\pi\left[\left(\beta / \beta_{G}\right)^{2}-1\right]} \sin \left(\frac{\pi N \beta_{G}}{2 \beta}\right)\right| \quad \text { if } N \text { is even; }
\end{aligned}
$$

For $\beta=\beta_{G}$, energy gain is:

$$
\Delta u(N)=\frac{G}{2} \cdot \frac{N \beta_{G} \lambda}{2} .
$$

The calculated energy gain per cavity (normalized to maximum energy gain in 11-cell) versus relative particle velocity (normalized to geometrical velocity) is shown for different number of cells in Figure 3.1. As expected, the energy gain per cavity is proportional to number of cells in a cavity. Energy gain per cavity can be increased even for lower number of cells if $\mathrm{RF}$ wavelength is increased.

- Operating range of cavity: In an ion linac, with kinetic energy ranging from mega electron volt $(\mathrm{MeV})$ to few giga electron volt $(\mathrm{GeV})$, beam velocity changes significantly after passing through each RF cavity, unlike an electron accelerator where particle relative velocity $\beta \sim 1$ remains almost constant even with further acceleration As a result, interaction between ions and RF cavity depends on the speed of ions. Thus, in an ion accelerator, cavity can be operated efficiently only for certain range of velocity of particle.

In Figure 3.2 energy gain (normalized to maximal energy gain of cavity versus relative particle velocity (normalized to $\beta_{G}$ ) is plotted for different number of 


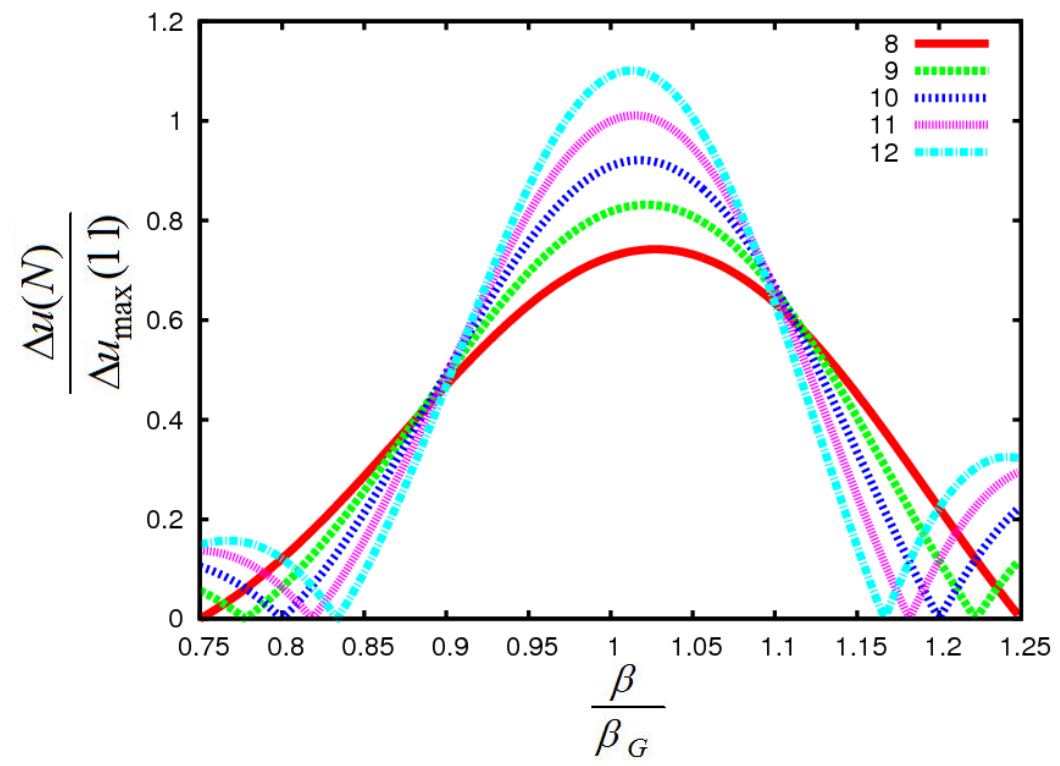

Figure 3.1: Energy gain per cavity - Maximum energy gain increases with increase in number of cell in multi-cell cavity.

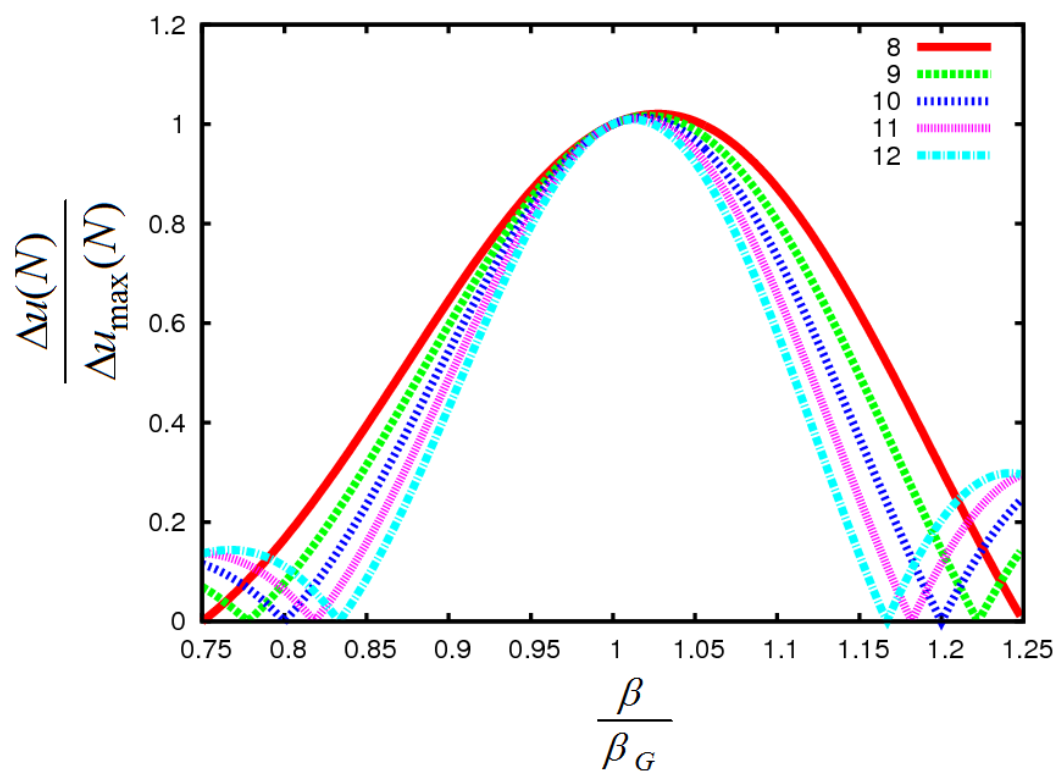

Figure 3.2: Operating range of cavity - Cavity with smaller number of cells is efficient for wider operating range. 


\section{DESIGN OF SCRF CAVITY FOR THE PROJECT-X LINAC}

cells. It is found that acceleration is maximum for particle velocity close to $\beta_{G}$ i.e., $\left(\beta / \beta_{G}\right) \sim 1$. The velocity acceptance for which cavity is operated efficiently, is governed by number of cells in a cavity. Smaller the number of cells, higher the velocity acceptance of cavity which leads to wider operating range of a cavity.

It can also be noticed from Figures 3.1 and 3.2 that maximum energy gain is not at $\beta / \beta_{G}=1$ but at a slightly higher value of $\beta / \beta_{G}$. It means that optimal performance of cavity does not come at designed beta but at higher beta. Deviation of optimal beta with respect to $\beta_{G}$ can be calculated as follows:

$$
\text { Let, } \quad \frac{\beta_{G}}{\beta}=x ; \quad \& \quad \frac{\pi . N}{2}=\alpha ;
$$

Energy gain for odd number of cells (equation (3.3)) can be expressed in terms of $\alpha$ and $x$ as under:

$$
\frac{\Delta u(N)}{u_{0}}=\left\|\frac{4}{N \pi} \cdot \frac{\cos (\alpha x)}{\left[1-x^{2}\right]}\right\| ;
$$

where $\mathrm{u}_{0}$ is energy gain at design beta. Differentiating equation (3.6) with respect to $x$ at $\beta=\beta_{\text {optimum }}$ results:

$$
\begin{gathered}
\left.\frac{\partial}{\partial x}\left(\Delta u(N) / u_{0}\right)\right|_{\beta=\beta_{\text {optimal }}}=0 ; \quad x=1-\left.\delta x\right|_{\beta=\beta_{\text {optimal }}} ; \\
\frac{1}{6} \alpha^{3}(\delta x)^{2}-\frac{2}{3} \alpha^{3}(\delta x)+\alpha=0 ;
\end{gathered}
$$

Since $\delta x$ is small so equation (3.8) can be solved using only first order term in $\delta x$ and neglecting second order term. Solution of equation for the first order of approximation is given as:

$$
\begin{aligned}
\delta x_{0} & \approx \frac{3}{2 \alpha^{2}}=\frac{6}{\pi^{2} \cdot N^{2}} \\
\beta_{\text {optimal }} & =\beta_{G}+\beta_{G} \frac{6}{\pi^{2} \cdot N^{2}}
\end{aligned}
$$

It can be noticed from equation (3.10) that deviation of $\beta_{\text {optimal }}$ with respect to $\beta_{G}$ is inversely proportional to square of number of cells in a cavity. Larger the number of cells, lower will be difference between $\beta_{\text {optimal }}$ and $\beta_{G}$ and hence cavity will perform optimum close to $\beta_{G}$.

Thus, the number of cells in cavity is a compromise between maximum energy gain and operating range of cavity. Usually choice between the two are made on the 
basis of operational requirements. Depending on the energy range and possible achievable accelerating gradient, an optimum value of the $\beta_{G}$ and number of cells in a cavity can be determined.

\subsubsection{Practical aspects}

SCRF cavity is a very expensive device in terms of material, technology, mechanical and chemical processing, and conditions of work: vacuum, liquid helium, adverse effects of outside magnetic fields, etc. Thus, involvement of technology and engineering makes design of SCRF cavity very critical. Main practical concerns which can influence cavity design are summarized below.

- Industrial production yield: To reduce the total building cost of accelerator, it is required to achieve high industrial yield of cavity fabrication. It is the ratio of number of cavities which pass operational requirement to the total number of cavities produced. A complicated design of cavity may influence industrial production yield. For instance, presence of large number of cells make the RF cavity more sensitive for possibility of chemical residual and post processing difficulties. Thus, industrial production yield of cavity with large number of cells is expected to be low. Large cavity aperture (iris radius) and large cavity wall slope assists to improve post processing difficulties. Large wall slope also improve mechanical stability of cavity.

- Technological constraints: To reduce the cost and time of SCRF cavity fabrication and its components, it is reasonable to operate with available technology. The major technological constraints include availability of RF source (Klystron), power coupler, HOM coupler and girder or cryomodule. RF source is also expensive device and its availability may influence operating frequency of cavity while number of cells (cavity length) can be influenced by availability of cryomodule design.

\subsubsection{Design of inner cell}

An inner cell is made of two identical half cells as shown in Figure 3.3 . Because of symmetry, only one quarter of an inner cell is used for optimization of cavity shape. Topology of cavity geometry is shown in Figure 3.3b. The quarter cell is composed 


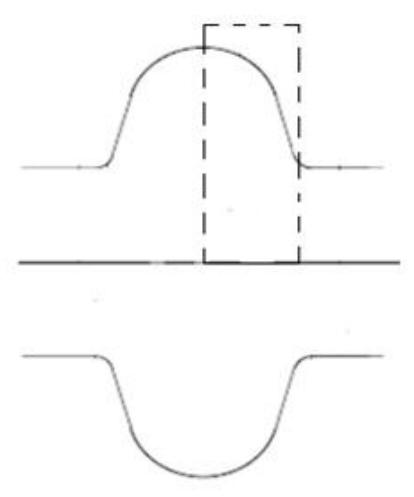

(a)

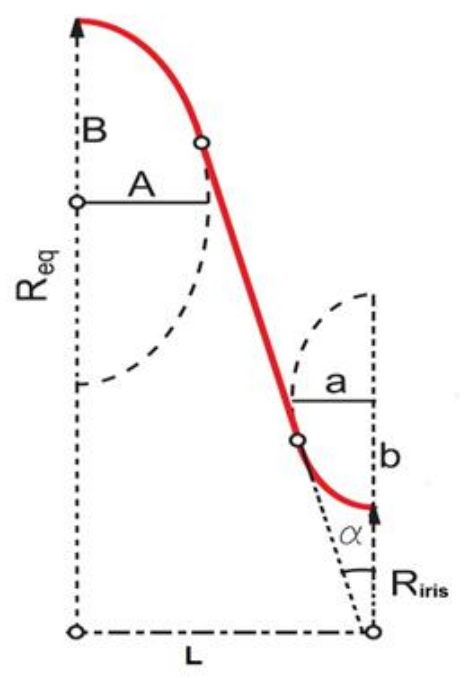

(b)

Figure 3.3: (a) Inner cell cavity with planes of symmetry (b) Segmented quarter of an inner cell. Equatorial portion is represented by $A, B$ and $R_{e q}$ and iris portion is shown by $a, b$ and $R_{\text {iris }}$.

of two elliptical arcs i.e., equatorial elliptical arc with semi-axes $A$ and $B$; and iris elliptical arc with semi-axes $a$ and $b$. Both elliptical arcs are joined by a straight line which is common tangent to both arcs, resulting in the wall inclination angle $\alpha . \quad R_{e q}$ is the equatorial radius (or cavity radius), $R_{\text {iris }}$ is the iris radius and $L$ is the length of the half cell. As given in equation (2.22) cell length $(2 L)$ is fixed after deciding operating frequency and reference particle velocity $\left(\beta_{G}\right)$. In order to obtain an optimal shape, different cells are designed for opted frequency by varying cavity geometrical parameters $A, B, a, b R_{\text {iris }}, R_{e q}$ and $\alpha$. In general, shape optimization is a difficult process because an improvement in one RF parameter usually results in degradation in another parameter. These shapes are compared to find an optimal solution in geometrical parameter space using following criteria:

Inter cell-to-cell coupling : RF power is delivered to multi-cell cavity by single power coupler. Thus, it is necessary to obtain reasonable inter cell-to-cell coupling for the operating mode to achieve uniform field amplitude in each cell. Inter cell-to-cell 
coupling is measured in term of coupling coefficient $(k)$ [58, which is defined as follows:

$$
k=2 \cdot \frac{f_{\pi}-f_{0}}{f_{\pi}-f_{0}} 100 \%,
$$

where $f_{\pi}$ and $f_{0}$ are operating mode frequency and zeroth mode frequency respectively. Inter cell-to-cell coupling also determines field profile sensitivity to cell frequency errors due to fabrication and mechanical deformations. The field non uniformity $\frac{\delta E}{E}$ i.e., fractional change in electric field in cells) in multi cell cavity for $N$ number of cells is given by the following relation [59]:

$$
\left(\frac{\delta E_{i}}{E}\right) \sim \frac{\Delta f_{i}}{f_{\pi}} \cdot \frac{N^{\frac{3}{2}}}{k},
$$

where $\frac{\Delta f_{i}}{f_{\pi}}$ is relative frequency error for fundamental mode in $\mathrm{i}^{\text {th }}$ cell. It can be noticed from equation 3.12 that for a given relative frequency error, $k$ should increase with a increase in number of cells to maintain same field flatness in the cavity.

Minimization of field enhancement factors: Ratio of peak surface fields $\left(E_{p}\right.$, $\left.H_{p}\right)$ to average accelerating field $\left(E_{a c c}\right)$ are called field enhancement factors. $E_{p} / E_{a c c}$ and $H_{p} / E_{a c c}$ are called electric and magnetic field enhancement factor respectively. $H_{p}$ in cavity occurs around equator region while $E_{p}$ occurs near iris region. Minimizing field enhancement factors for achieving higher gradient in a cavity is an important aspect of cavity design. It also helps in improving interaction between beam and cavity.

Optimization of $R / Q$ and Geometrical factor: As discussed in section 2.4, cavity wall losses reduce with increase in product of geometrical factor $(G)$ and $R / Q$. Thus, shape is designed to obtain optimal magnitude of their product $G$. $(R / Q)$. These parameters are independent of material property and depend only on cavity geometry and hence, are very useful parameters to compare two geometries.

\subsubsection{Influence of geometrical variables}

Iris radius $\left(R_{\text {iris }}\right): \quad \mathrm{R}_{\text {iris }}$ is a very important parameter which influences several cavity RF parameters. In multi-cell elliptical cavity, cell to cell coupling takes place via the electric field through the irises. Variation in $k$ with $R_{\text {iris }}$ can be seen in Figure 3.4 for a given set of other parameters. It can be noticed that inter cell-to-cell coupling would increase with increase in $R_{\text {iris }}$ of cavity. Figure 3.5a shows variation in field enhancement factors with $R_{\text {iris }}$ and it is clear that enhancement factors also increase 


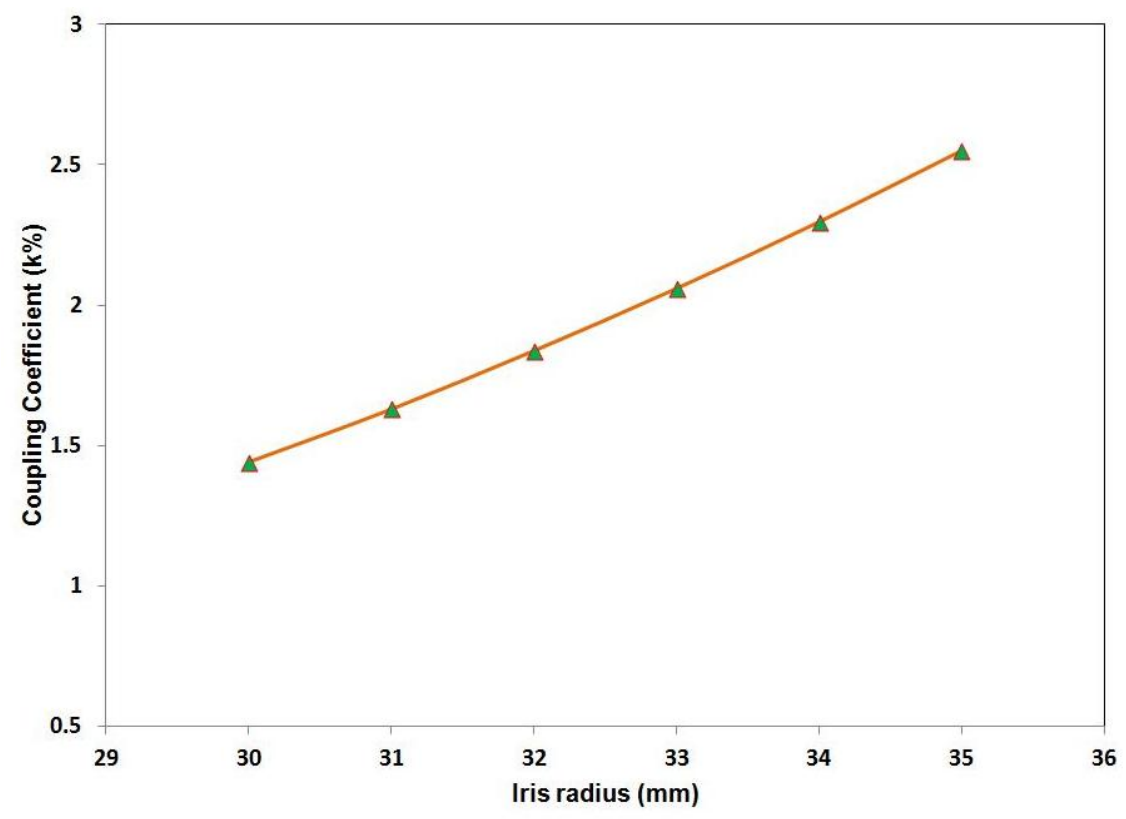

Figure 3.4: $\mathbf{R}_{\text {iris }}$ and coupling coefficient - Coupling coefficient increases with increase in iris radius.

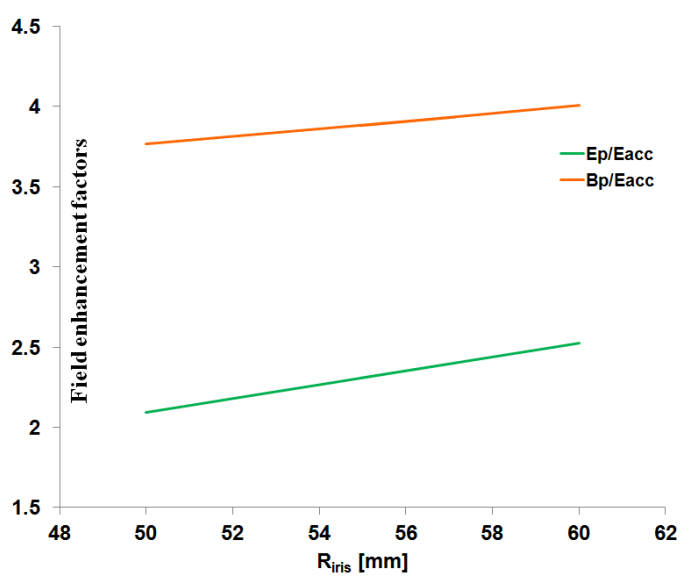

(a)

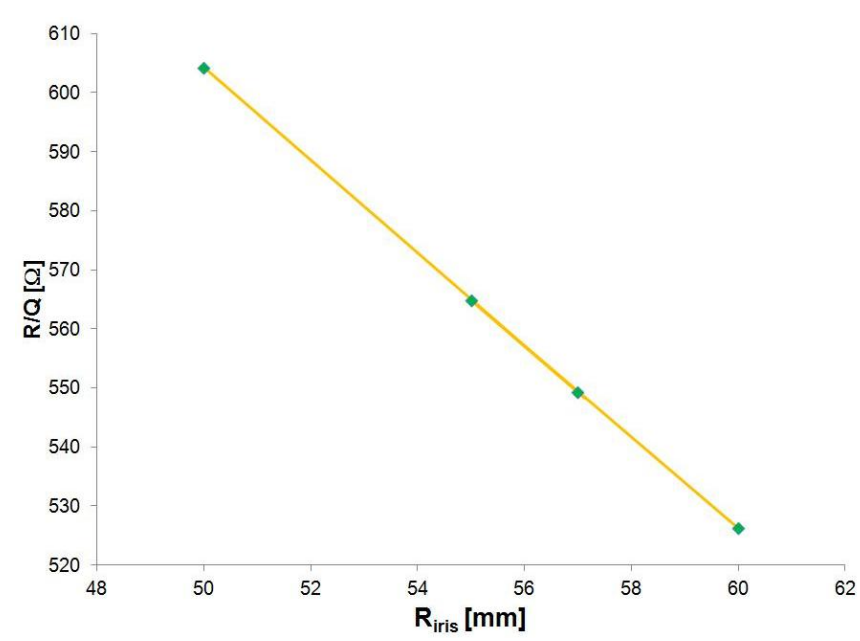

(b)

Figure 3.5: Variation in field enhancement factors and $R / Q$ with iris radius Field enhancement factor increases with increase in $R_{\text {iris }}$ but the $R / Q$ decreases linearly with increase in iris radius. 
with increase in $\mathrm{R}_{\text {iris }}$. Longitudinal electric field is more focused on axis for smaller $R_{\text {iris }}$ and hence $R / Q$ is higher for smaller $R_{\text {iris }}$ which can be noticed in Figure $3.5 \mathrm{~b}$. Therefore, normal conducting cavities are built with smaller $R_{\text {iris }}$ to obtain higher $R / Q$ which results in reduction of power dissipation to cavity wall but in SCRF cavities, $R / Q$ can be sacrificed to avoid wake effects. Thus, $R_{\text {iris }}$ is a critical geometrical parameter and its correct scaling is necessary to achieve optimal cavity shape. Large $R_{\text {iris }}$ is preferred for high current application due to reduced beam losses and simplifying HOMs damping.

Equatorial radius $\left(R_{e q}\right)$ : It is very sensitive to the frequency of the fundamental mode and it marginally affects the electromagnetic and mechanical properties. Hence tuning the frequency of the cell is performed by varying $R_{e q}$ without changing any of the other independent geometrical parameters i.e., $L, R_{\text {iris }}, A, B$, $a$ and $b$.

Wall inclination angle $(\alpha)$ : A straight line tangent on two elliptical arc makes an angle with iris plane which is called wall inclination angle. It is preferred to design a cavity with large wall inclination angle as it avoids the possibilities of chemical and water residuals in cavity. Smaller wall inclination angle may result in chemical residual which can degrade cavity performance. It also affects mechanical stability of cavity. Stresses (due to helium vapour pressure) on a cavity at $2 \mathrm{~K}$ are localized for small $\alpha$ and uniformly distributed for large $\alpha$. If stresses are higher than elastic limit of material, it may result in permanent mechanical deformation and degrade cavity performance. For fixed cell length, wall inclination angle strongly depends on $A$ and $a$.

Semi axes of equatorial ellipse $(\boldsymbol{A}, \boldsymbol{B})$ : To understand the influence of semi axes of equatorial elliptical on the RF parameters of cavity, simulation is performed. The geometry which is used in simulation is summarized in Table 3.1 The influence of semi axes of equatorial elliptical arc on field enhancement factors and R/Q is shown in Figure 3.6. It can be noticed that electric field enhancement factor weakly depends on aspect ratio of equatorial elliptical arc. For the fixed value of $L, R_{\text {iris }}, A, a$ and $b$, variation in parameter $B$ has no significant influence on either magnetic field enhancement factors or $\mathrm{R} / \mathrm{Q}$. However, variation in $A$ (other geometrical parameters are fixed except $\left(R_{e q}\right.$ and $\alpha$ ) results in significant changes in magnetic field enhancement factor and $R / Q$. Magnetic field enhancement factor decreases with increase in $A$ due to enhancement of magnetic volume of the geometry. There is also significant improvement in $R / Q$ with increase in $A$. 


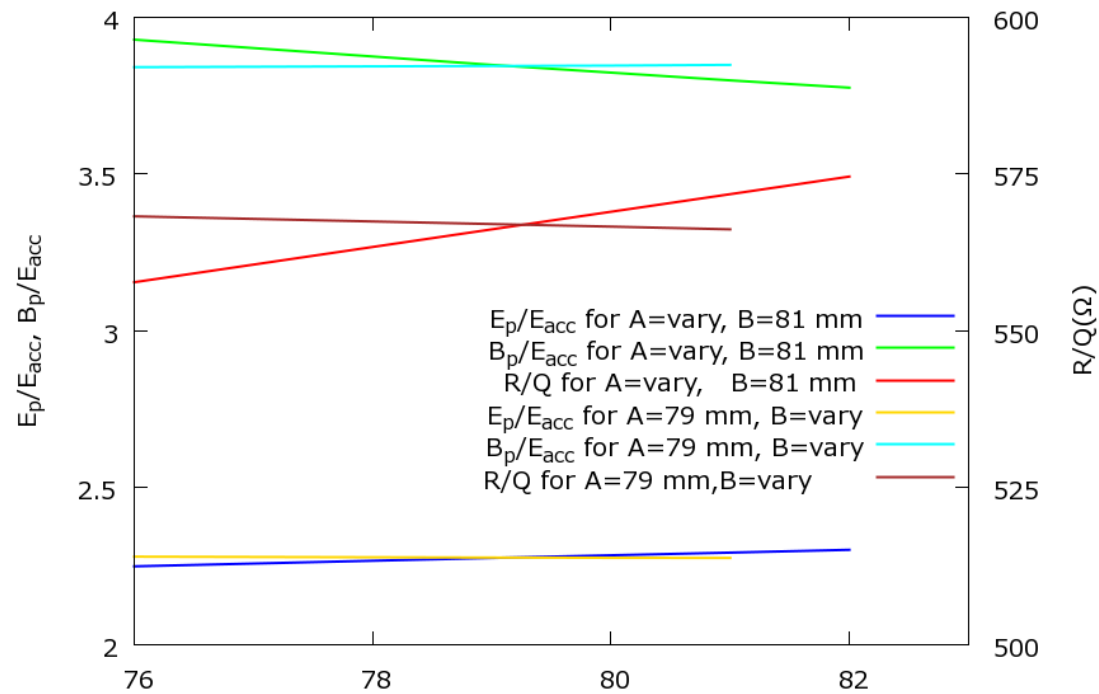

A, B (mm)

Figure 3.6: Influence of semi axes $\mathrm{A}$ and $\mathrm{B}$ of equatorial elliptical arc - Magnetic field enhancement factor and $\mathrm{R} / \mathrm{Q}$ vary with $A$ but almost constant with $B$.

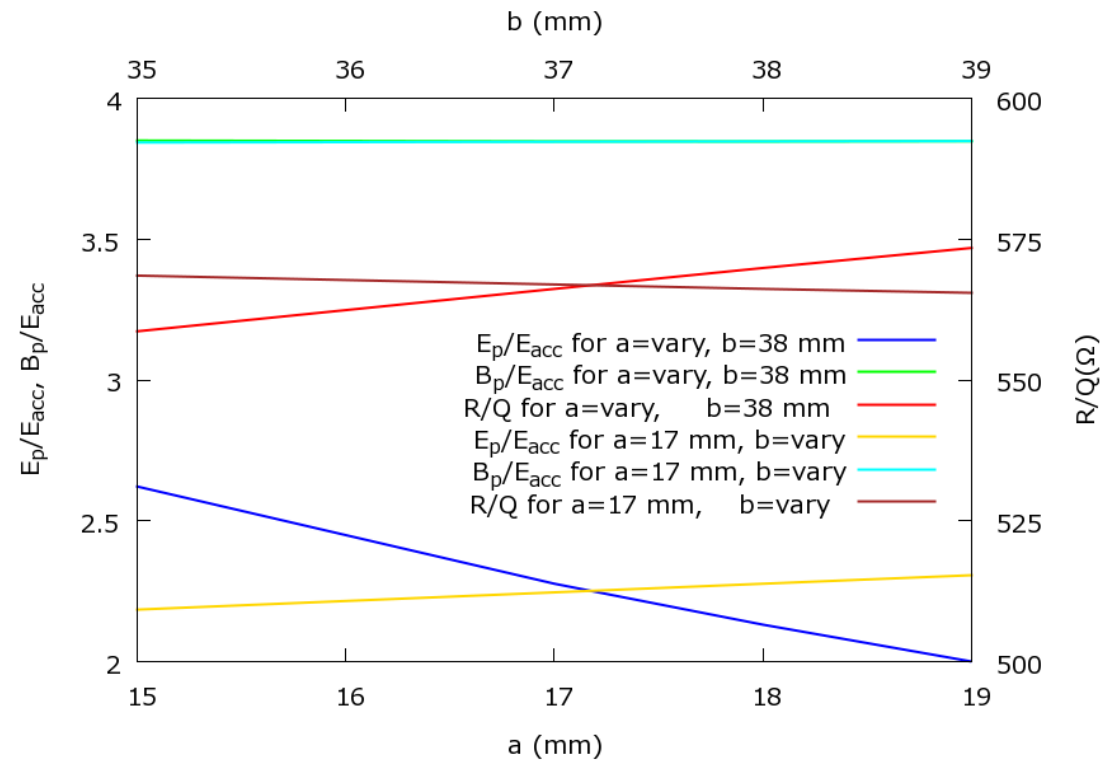

Figure 3.7: Influence of semi axes $a$ and $b$ of iris elliptical arc - Electric field enhancement factor strongly influenced by iris aspect ratio. 
Table 3.1: Geometry used to understand influence of geometrical parameters of cavity.

\begin{tabular}{lcc}
\hline Parameters & Unit & Magnitude \\
\hline $\mathrm{R}_{\text {iris }}$ & $\mathrm{mm}$ & 50 \\
$\mathrm{~A}$ & $\mathrm{~mm}$ & 79 \\
$\mathrm{~B}$ & $\mathrm{~mm}$ & 81 \\
$\mathrm{a}$ & $\mathrm{mm}$ & 17 \\
$\mathrm{~b}$ & $\mathrm{~mm}$ & 38 \\
$\mathrm{~L}$ & $\mathrm{~mm}$ & 103.75 \\
$\alpha$ & degree & 9.4 \\
\hline
\end{tabular}

Semi axes for iris ellipse $(a, b)$ : Electric field enhancement factor is mainly determined by the aspect ratio of elliptical arc at iris. Variation of RF parameters with semi axes of iris elliptical arc is shown in Figure 3.7. It can be concluded that magnetic field enhancement factor is independent of aspect ratio $(a / b)$ of iris elliptical arc. Within the studied range of parameters, increase in $a$ or decrease in $b$ decreases electric field enhancement factor. It can also be observed that dependence of electric field enhancement factor and $R / Q$ are relatively strong on $a$ than on $b . R / Q$ also improves with increase in $a$ and it is weakly dependent on changes in $b$.

Table 3.2: A summary of cavity design procedure

\begin{tabular}{llll}
\hline Criterion & Parameter & $\begin{array}{l}\text { Optimization } \\
\text { goals }\end{array}$ & $\begin{array}{l}\text { Geometrical } \\
\text { Parameter }\end{array}$ \\
\hline Field flatness & $\mathrm{k}$ & $\uparrow$ & $\mathrm{R}_{\text {iris }} \uparrow$ \\
High gradient & $\mathrm{E}_{p} / E_{\text {acc }}, \mathrm{H}_{p} / E_{\text {acc }}$ & $\downarrow$ & $\mathrm{R}_{\text {iris }} \downarrow, \mathrm{A} \uparrow, \mathrm{a} \uparrow$ \\
Low cryogenic & $\mathrm{R} / \mathrm{Q} \cdot \mathrm{G}$ & $\uparrow$ & $\mathrm{R}_{\text {iris }} \downarrow, \mathrm{A} \uparrow, \mathrm{a} \uparrow$ \\
loads & & & \\
Low HOMs & $(\mathrm{R} / \mathrm{Q})_{H O M}$ & $\downarrow$ & $\mathrm{R}_{\text {iris }} \uparrow$ \\
impedance & & & \\
\hline
\end{tabular}

Table 3.2 summarizes typical goals of shape optimization procedure. First column shows criterion and second column shows parameter which influences criterion. Third column gives goal of optimization while fourth column shows geometrical parameters which influence respective parameters and its variation to achieve optimization goal. In Table 3.2 the upper arrow $(\uparrow)$ indicates increase while down arrow $(\downarrow)$ indicates 


\section{DESIGN OF SCRF CAVITY FOR THE PROJECT-X LINAC}

decrease and parameters have their usual meaning.

\subsubsection{End cell design}

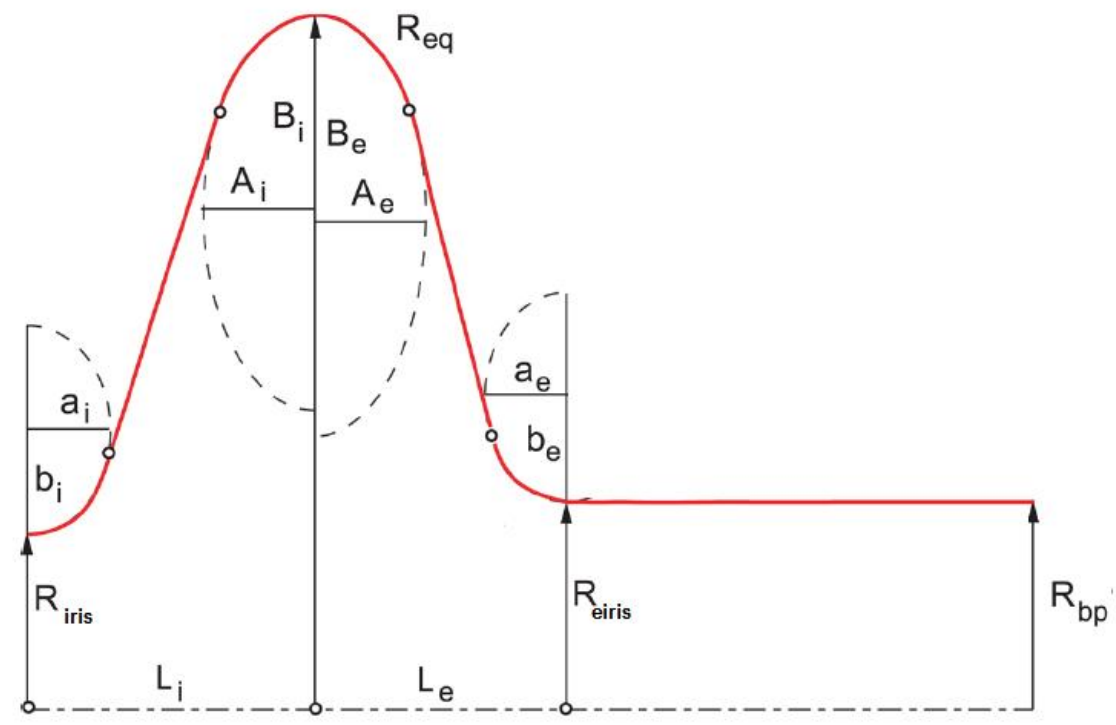

Figure 3.8: A typical end cell design- End cell is composed of half inner cell (left) and half outer (right) cell with beam pipe.

A typical end cell is designed with two different half cells. The inner half cell and outer half-cell. Inner half is identical to regular cell but outer half cell is needed to design differently as it is attached with beam pipe to allow the beam to pass through it. A typical end cell design is shown in Figure 3.8. One can use the same topology for end cell geometry as used for the inner cell, i.e., two geometrical arcs connected with a common tangent. Since regular cell is already designed so we need to design only outer half of the end cell. $R_{e q}$ is fixed by the design of the inner cell. Thus, to maintain same frequency, shape of the half end-cell is tuned in a different way. Frequency of the outer half end cell is tuned by varying the length of the half-cell, which is connected to the beam pipe, while keeping $R_{e q}$ fixed. Optimization of end cell is dictated by following considerations:

- Because of different shape of end cell, coupling between end cell and regular cell can differ from the coupling between adjacent inner cells. It may result in 
difference in field amplitude in end cells. Field flatness (FF) factor is used to measure field flatness of cavity. For operating mode, it can be expressed as:

$$
F F(\%)=100 \cdot \frac{E_{c \max }-E_{c m i n}}{\frac{1}{N} \cdot \Sigma_{i=1}^{N} E_{c i}} ;
$$

where; $E_{c \max }$ and $E_{c m i n}$ are maximum and minimum of peak fields in a cavity, $E_{c i}$ is peak field in $i^{t h}$ cell and $N$ is number of cells in a cavity. To optimize the net acceleration through the cavity, it is necessary to achieve uniform field distribution in each cell for a given stored energy. Field flatness factor is minimized during optimization process and tried to keep as low as possible (usually less than $2 \%$ ). However in reality, field flatness of cavity deteriorates due to fabrication errors and field flatness factor is much higher $(\sim 5 \%)$ than designed value.

- In order to avoid field emission and other limiting effects in cavity, magnitude of field enhancement factors in end cell should not differ too much from inner cell. Typically, end cells are designed with field enhancement factors less than those in inner cell.

\subsubsection{HOMs damping requirement}

Fields induced by HOMs act back on the beam and result in beam instability in longitudinal as well as transverse directions. Trapped HOMs are those modes of concern whose electric field amplitude is concentrated within the inner cells. They have very low amplitude within the end cells so the electromagnetic field decays in beam pipe before reaching the HOM coupler. Hence these modes are usually weakly coupled with the HOM coupler and remain localized within the cavity. As a result the energy corresponding to trapped modes is accumulated within the RF cavity and it reaches a considerable value after passage of several bunches. It increases cryogenic losses and also causes beam instability. The main reasons which result in trapping of modes in cavity are as under.

- Number of cells in cavity: Large number of cells in cavity makes it more sensitive toward the trapping of HOMs. It is essential to achieve reasonable cell to cell coupling not only for fundamental mode but also for HOMs too so that they can propagate throughout each cell in the cavity. 


\section{DESIGN OF SCRF CAVITY FOR THE PROJECT-X LINAC}

- End cell design: Because of different shape of end cell from inner cell, coupling between end cell and regular cell can differ from the coupling between adjacent inner cells. If this difference is too large, some modes can be reflected back and get trapped within inner cells only. In the absence of any potential trapped modes, same end cell can be used at both ends. This cavity is usually called symmetric cavity. However, different end cells are used for both ends in the presence of trapped modes so that if one mode is trapped form one side, it can be extracted from other side.

- Beam pipe radius: HOMs below the cutoff frequency of the beam pipe decay exponentially and sometimes they decay very fast that can not propagate into the beam pipe. Thus, it is necessary to have beam pipe diameter large enough to propagate all HOMs while staying sufficiently below the cutoff of the fundamental mode.

\subsection{Design of SCRF cavity for the pulsed linac}

Initial proposal for the Project-X facility was based on the SC pulsed linac [20]. Layout of pulsed SC linac for Project-X facility is shown in Figure 3.9. It can be noticed that

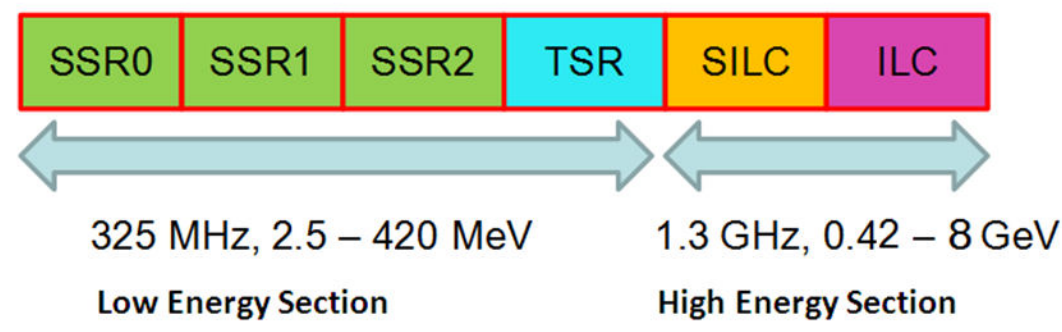

Figure 3.9: Project-X pulse SC linac-Scheme of acceleration.

SC linac is segmented into two parts on the basis of energy; low energy and high energy part. The low energy part uses four families of spoke resonators. First three based on single spoke resonators (SSR) and last one on triple spoke resonator (TSR). All are operated at frequency of $325 \mathrm{MHz}$. The high energy part, which is used for the acceleration of the beam from $420 \mathrm{MeV}$ to $8 \mathrm{GeV}$, includes a squeezed International Linear Collider (SILC) section which uses a squeezed ILC type geometrical beta $\left(\beta_{G}\right)$ 
$=0.81,1.3 \mathrm{GHz}$ cavity for acceleration of the beam from 0.42 to $1.2 \mathrm{GeV}$ and an ILC section which uses an ILC type $\left(\beta_{G}\right)=1,1.3 \mathrm{GHz}$ cavity for the rest of the linac. The requirements for the $1.3 \mathrm{GHz}$ section are summarized in Table 3.3 .

Table 3.3: Basic parameters of $1.3 \mathrm{GHz}$ section of linac.

\begin{tabular}{ccc}
\hline Parameters & Unit & Magnitude \\
\hline Average gradient in ILC section & $\mathrm{MV} / \mathrm{m}$ & 25 \\
Average gradient in SILC section & $\mathrm{MV} / \mathrm{m}$ & 23 \\
Pulse length & $\mathrm{ms}$ & 1.25 \\
Repetition rate & $\mathrm{Hz}$ & 5 \\
Initial Energy & $\mathrm{MeV}$ & 420 \\
Energy after SILC & $\mathrm{MeV}$ & 1200 \\
Energy after ILC & $\mathrm{MeV}$ & 8000 \\
\hline
\end{tabular}

A cumulative experience of the SCRF community was applied to the development of the SC, $1.3 \mathrm{GHz}$, ILC-type cavity for the TESLA project [60, 61]. The resulting elliptical shaped cavity was considerably well optimized for highly relativistic particles i.e., $\beta \sim 1$ and was supposed to be used for the ILC section, Thus, initial work in high energy section was related with the design of SILC cavities. Many of the problems associated with the choice of cavity shape for the SILC section can be overcome by adopting similar topology as used for ILC section; however length of cavity and number of cells in a multi-cell cavity are decided by operational requirements. $\beta_{G}=0.81,1.3$ $\mathrm{GHz}, 11$-cell cavity is designed for SILC section of linac. The final design is optimized on the basis of following constraints:

- Availability of cryomodule: It was considered to utilize existing design of the ILC cryomodule for the SILC section, which is Type-4 ILC cryomodule [62, 63]. It is proposed to use eight cavities in all cryomodules as in ILC design. In such situation, position of each cavity is fixed by coupler slot. Thus, efficiency of accelerator not only depends on cavity length and its velocity acceptance but also on the spacing between two successive cavities. The length of $\beta_{G}=0.81$, 11-cell cavity is same as the ILC $\beta_{G}=1$, 9-cell cavity. A longer cavity (number of cells greater than 11) does not fit in cryomodule and shorter cavity (number of cells smaller than 11) does not occupy all available space. Thus, design of cavity with 11-cell leads to an optimal solution. Proposal of using same cryomodule 


\section{DESIGN OF SCRF CAVITY FOR THE PROJECT-X LINAC}

as in ILC section allows one to use same scheme of power distribution in both sections. One $10 \mathrm{MW}$ klystron with every two cryomodules is used to feed RF power in high energy section of the linac.

- Availability of power coupler and other components: Power requirements for 11-cell, SILC cavity is also similar to ILC $\beta_{G}=1$ cavity, thus, it is possible to use same power coupler and HOM coupler. It also allows using similar type of other auxiliary components like tuners etc.

All the above considerations lead to a choice of 11-cell cavity [20, 56, 57] for the SILC section. This cavity was designed for symmetrical end cells which means end cells at both ends are similar. Figure 3.10 shows a 11-cell cavity with power and HOM couplers. $\mathrm{RF}$ parameters and geometrical parameters for optimized structure are summarized in Table 3.4

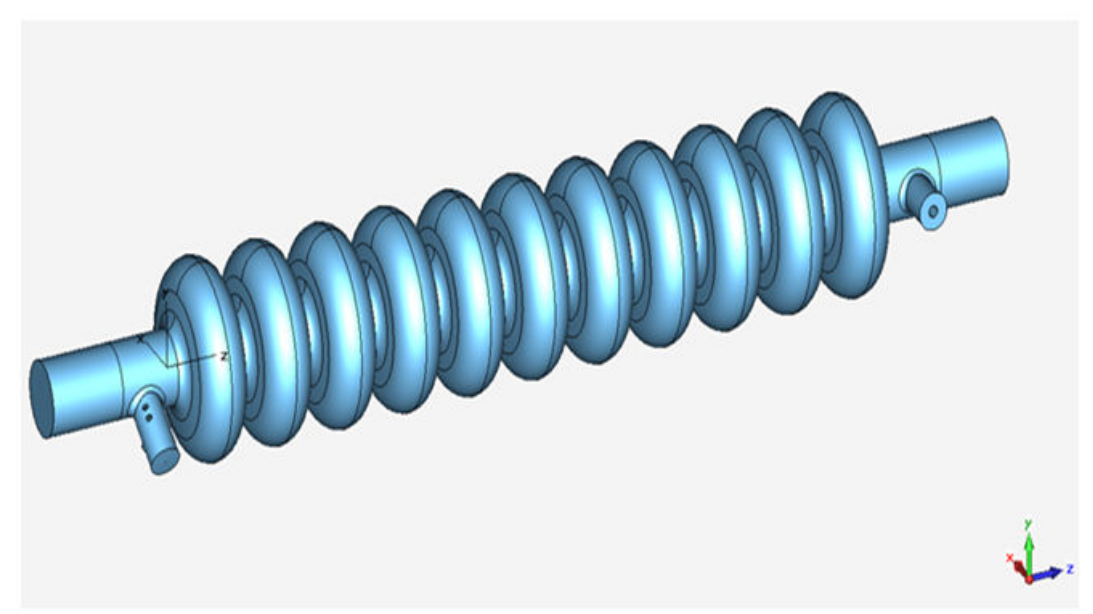

Figure 3.10: Configuration of 11 cell cavity with coupler.

In the table, $\alpha_{i n n e r}$ and $\alpha_{\text {end }}$ are wall inclination angle for inner cell and end cell respectively.

\subsubsection{HOMs study for symmetrical 11-cell SILC cavity}

In an ion linac, with kinetic energy ranging from $\mathrm{MeV}$ to few $\mathrm{GeV}$, beam velocity changes significantly after passing through each RF cavity. As a result, interaction between ions and RF cavity depends on the speed of ions. In SILC section $H^{-}$velocity 
Table 3.4: $1.3 \mathrm{GHz} \beta_{G}=0.81$ 11-cell cavity parameters

\begin{tabular}{lcc}
\hline Parameters & Unit & Magnitude \\
\hline$\alpha_{\text {inner }}$ & deg. & 4.4 \\
$\alpha_{\text {end }}$ & deg. & 8.6 \\
$\mathrm{R}_{\text {iris }}$ & $\mathrm{mm}$ & 36 \\
Beam pipe radius & $\mathrm{mm}$ & 39 \\
Active length & $\mathrm{mm}$ & 1031 \\
$k$ & $\%$ & 2.7 \\
$\mathrm{R} / \mathrm{Q}$ & $\Omega$ & 754 \\
Geometrical factor & $\Omega$ & 228 \\
$E_{p} / E_{a c c}$ & - & 2.4 \\
$B_{p} / E_{a c c}$ & $\mathrm{mT} /(\mathrm{MV} /$ meter $)$ & 5.0 \\
\hline
\end{tabular}

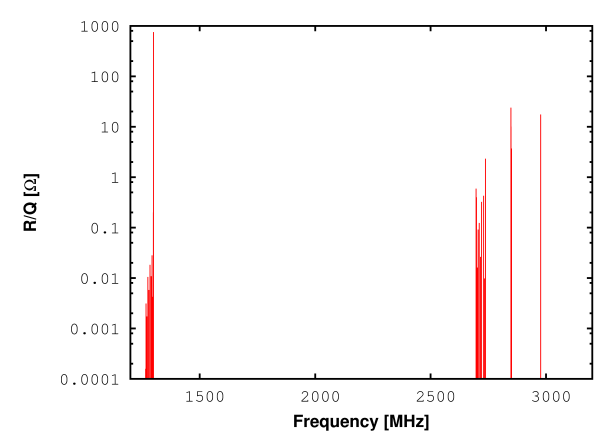

(a)

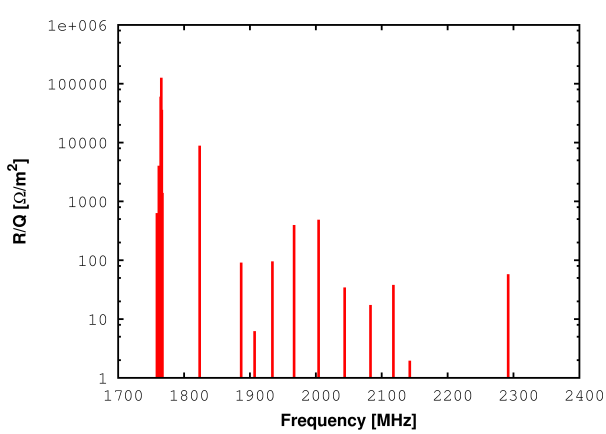

(b)

Figure 3.11: HOM spectrum for 11-cell cavity - Impedance of (a) monopole (Longitudinal) modes $\mathrm{R} / \mathrm{Q}$ and (b) Dipole (transverse) modes $(R / Q)_{\|}$are shown for $\beta_{G}=$ 0.81 .

changes from $\beta=0.70$ to 0.90 . Thus, symmetrical 11-cell cavity is studied for complete range of $\beta$ in order to investigate potentially trapped HOMs. Monopole modes and dipole modes are taken into account. Modes with frequency higher than cut-off frequency of beam pipe can easily propagate out of cavity. The effective impedance distribution $(R / Q)$ for monopole and dipole modes (below the cut-off frequency of the beam pipe) for $\beta_{G}=0.81$ cavities are shown in Figure 3.11. These spectrum provides a sight to concerned HOMs whose effective impedances are high and frequencies are 


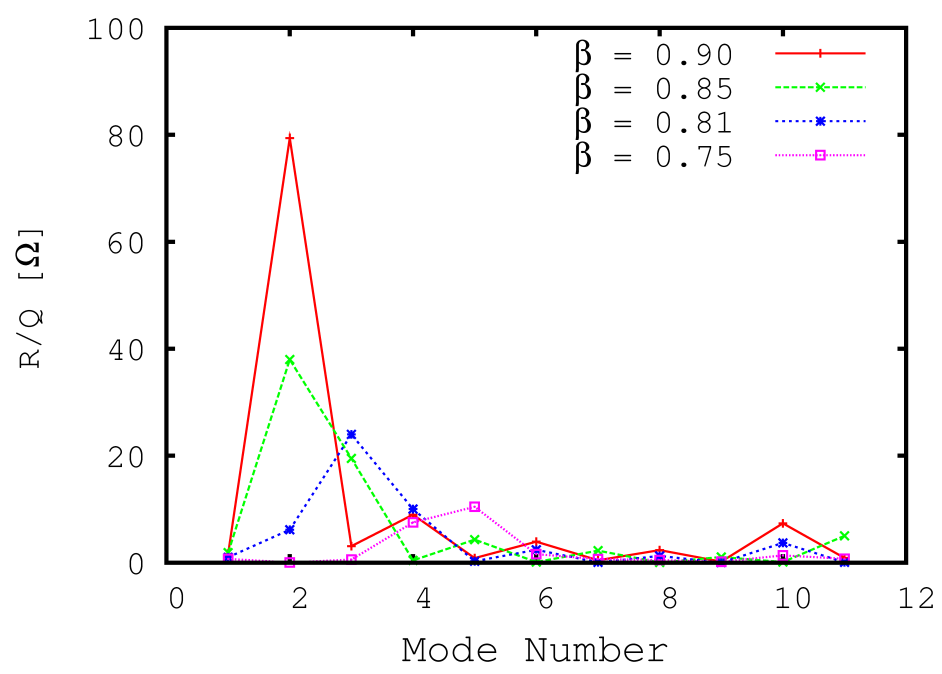

Figure 3.12: R/Q distribution of HOMs for third monopole passband of 11-cell cavity.

close to integral multiple of beam repetition rate (i.e., $325 \mathrm{MHz}$ for Project-X linac).

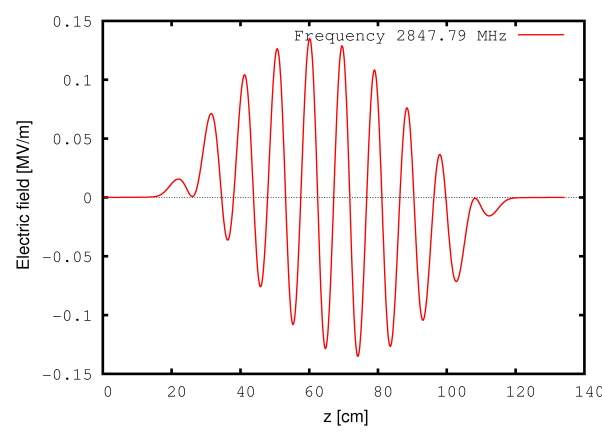

(a)

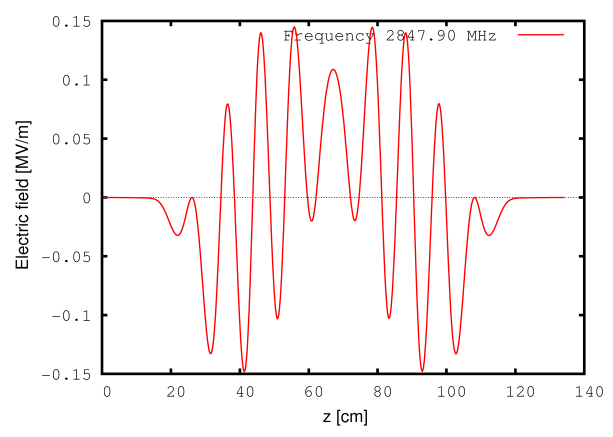

(b)

Figure 3.13: Trapped modes in 11-cell cavity - Electric field distribution along the axis of cavity for most concerned modes with resonance frequency of (a) $2847.79 \mathrm{MHz}$ and (b) $2847.90 \mathrm{MHz}$ for symmetric 11 cell cavity.

Monopole third pass band is found to be most sensitive toward trapped modes. Figure 3.12 shows the distribution of the HOMs effective impedance for the third pass band for the complete range of $\beta$. It can be seen that most concerned monopole mode 
corresponds to frequency of $2847.79 \mathrm{MHz}$ with highest effective impedance of $80 \Omega$ for $\beta=0.90$ and $40 \Omega$ for $\beta=0.85$. Next mode of concern corresponds to frequency of $2847.90 \mathrm{MHz}$ with highest effective impedance of $24 \Omega$ for $\beta=0.81$.

Figures $3.13 \mathrm{a}$ and $3.13 \mathrm{~b}$ show the electric field distributions for the two most concerned monopole modes along the axis of the cavity. For both of these modes, electric field is largely concentrated within inner cells while the amplitude at the end cells is relatively small. The results were also verified by using another RF simulation code HFSS [44. Figure 3.14 shows HFSS results of field distribution along the axis for mode with frequency $2847.79 \mathrm{MHz}$. Thus, the symmetric 11-cell cavity is found to be sensitive to trapped modes at monopole third pass band. Therefore, it is necessary to make some changes in cavity geometry to avoid trapping of HOMs.

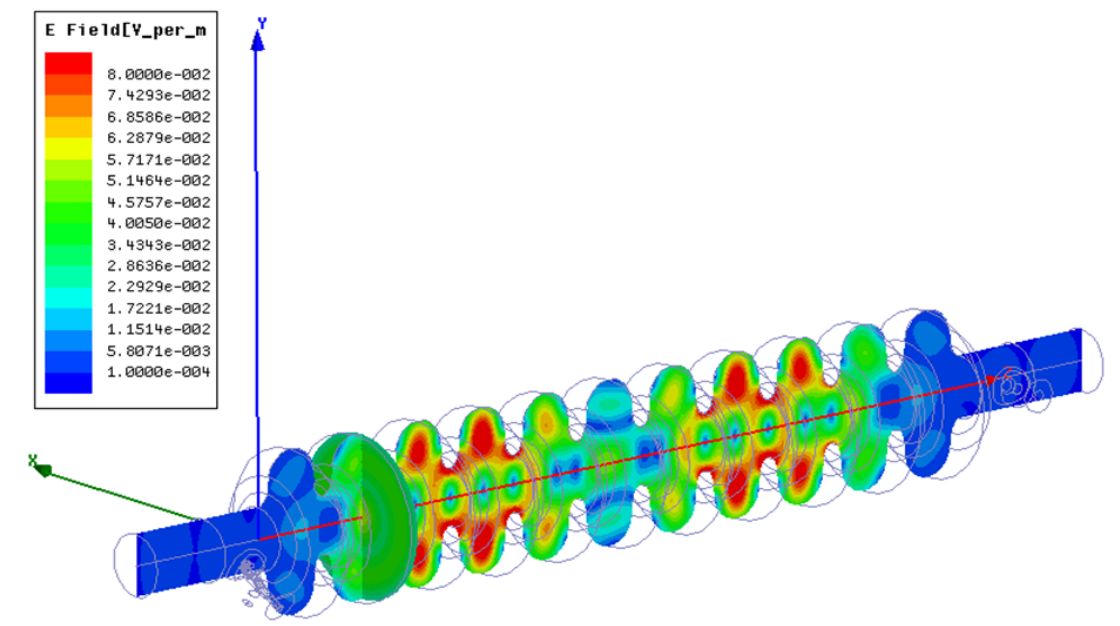

Figure 3.14: HFSS result confirms trapping of mode with frequency $2847.79 \mathrm{MHz}$.

Avoiding trapping of HOMs: Trapped modes may result in beam instability and cryogenic losses even they have low effective impedance. Therefore, it is essential to design a cavity which is free from trapped modes. In general trapping of HOMs can be avoided using following ways:

- Use of large beam pipe diameter: Increase in beam pipe diameter results in an increase in the coupling of modes with HOM coupler and also drop in cut-off frequency. Thus, modes can easily propagate out of cavity. However, it is not always possible to increase beam pipe diameter as it also results in field leakage for fundamental mode which causes increase in cryogenic losses. 


\section{DESIGN OF SCRF CAVITY FOR THE PROJECT-X LINAC}

- Asymmetrical end cells: Use of different end cell at each end of cavity (asymmetrical cavity) allows to get rid of trapped HOMs. Modes propagate outside the cavity from other end if it is trapped from one side. However, it increases the engineering efforts required to fabricate the cavity. It is required to make three types of Dye (inner cell, end cell right and end cell left) instead of two (inner cell and end cell) for half cell forming. Still, it seems preferable option to avoid trapping of HOMs because there is no need of compromise with field flatness of fundamental mode.

Thus, to avoid trapped modes, an asymmetrical design of the end-cells is proposed for 11-cell cavity without disturbing the field flatness of the fundamental mode. In order to do so one of the end cell of the 11-cell cavity is detuned a little by changing cell parameters of outer half. Shape of the end cell is optimized in such a way that the natural eigen mode frequency of end cell comes closer to the frequency of the most concerned mode (i.e., $2847.79 \mathrm{MHz}$ ). It helps in increasing the field amplitude of this mode at the end cell and, thus, increases coupling of the field amplitude with the HOM coupler. The field enhancement factor of the end cell is kept less than the field enhancement factor of the inner cell. After optimization, the natural eigen mode frequency spectrum of inner and end cells are shown in the Table 3.5. The asymmetrical

Table 3.5: Eigen mode frequency spectrum after end cell optimization.

\begin{tabular}{cccc}
\hline Pass band no. & Inner cell & End cell 1 & End cell 2 \\
\hline 1 & $1300 \mathrm{MHz}$ & $1300 \mathrm{MHz}$ & $1300 \mathrm{MHz}$ \\
2 & $2734.3 \mathrm{MHz}$ & $2732.4 \mathrm{MHz}$ & $2726.6 \mathrm{MHz}$ \\
3 & $2850.1 \mathrm{MHz}$ & $2851.6 \mathrm{MHz}$ & $2847.6 \mathrm{MHz}$ \\
\hline
\end{tabular}

11-cell cavity has been again studied for most concerned modes. The distributions of effective impedance of the monopole and dipole modes are shown in Figures 3.15a and $3.15 \mathrm{~b}$ respectively. The distribution of HOMs effective impedances (R/Q) for the third monopole pass band for complete range of $\beta$ is plotted in Figure 3.16 ,

Modes corresponding to frequencies $2847.74 \mathrm{MHz}$ and $2847.82 \mathrm{MHz}$ are most concerned ones. Mode for the frequency $2847.74 \mathrm{MHz}$ has highest effective impedance of $64 \Omega$ for $\beta=0.90$ and mode corresponding to frequency $2847.82 \mathrm{MHz}$ has $30 \Omega$ for the same $\beta$. Electric field distributions of these modes are illustrated in Figures 3.17a and 


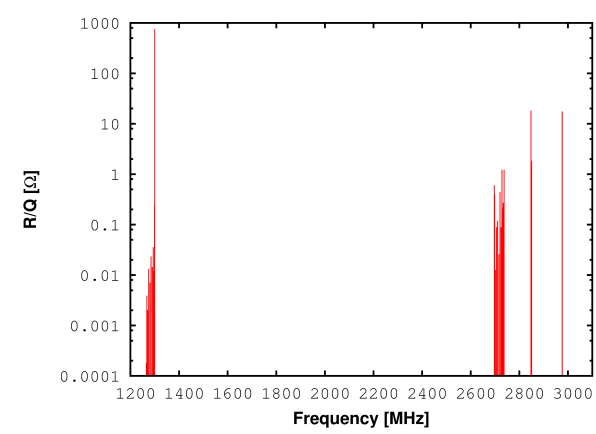

(a)

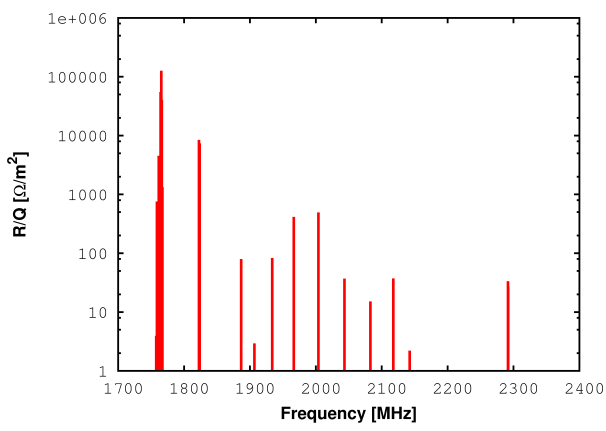

(b)

Figure 3.15: HOM spectrum for asymmetrical 11-cell cavity - Impedance of (a) monopole (Longitudinal) modes $\mathrm{R} / \mathrm{Q}$ and (b) Dipole (transverse) modes $(R / Q)_{\|}$are shown for $\beta_{G}=0.81$.

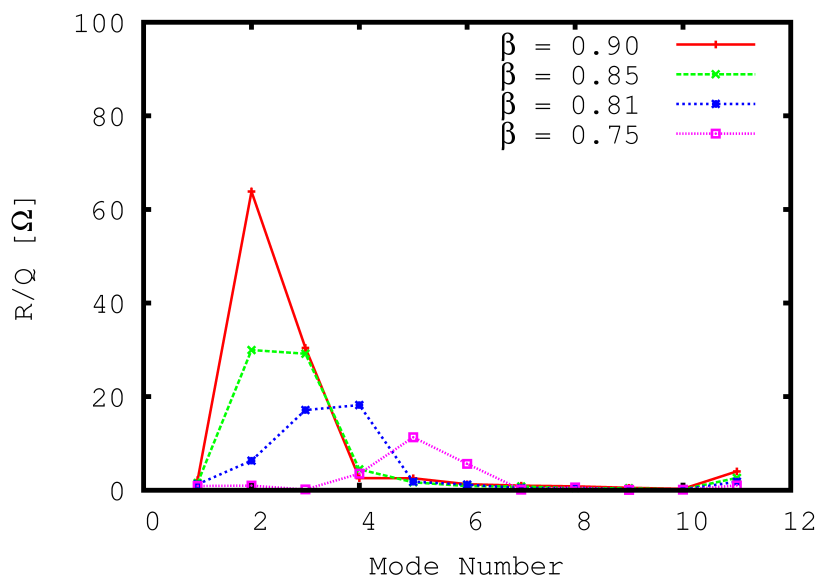

Figure 3.16: R/Q distribution of HOMs for third monopole passband of asymmetric 11-cell cavity.

3.17b. It can be seen that these modes are not trapped anymore because they have sufficient amplitude within at least one of the end cell to ensure their coupling with the HOM coupler.

The asymmetrical 11-cell cavity has been studied for the same configuration of couplers as the ILC type cavity i.e., two HOM couplers and one input coupler using 3 -dimensional code HFSS. External quality factors $\left(Q_{e x}\right)$ are calculated using complex 


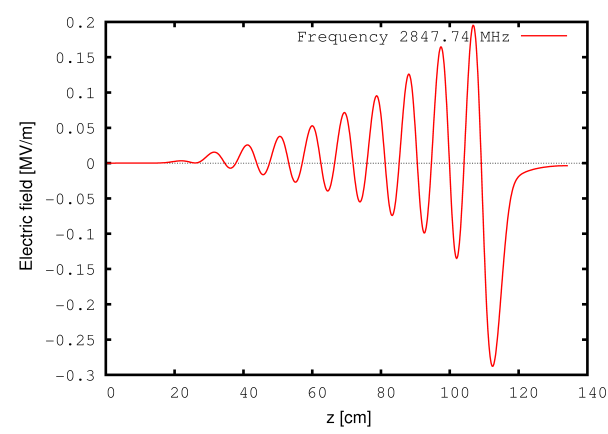

(a)

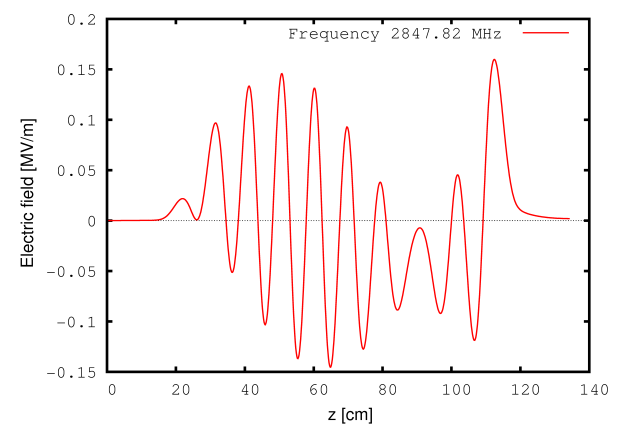

(b)

Figure 3.17: Most concerned modes in asymmetrical 11-cell cavity - Electric field distribution along the axis of cavity for most concerned modes with resonance frequency of (a) $2847.74 \mathrm{MHz}$ and (b) $2847.82 \mathrm{MHz}$ for asymmetric 11 cell cavity.

eigenmode solver with matched boundary conditions on all couplers outputs. $Q_{e x}$ of most concerned modes were calculated and compared with symmetrical cavity. Frequencies, $\mathrm{Q}_{e x}$ and $\mathrm{R} / \mathrm{Q}$ of typical monopole HOMs in third pass band for symmetrical and asymmetrical cavities are listed in Table 3.6 .

Table 3.6: $\mathrm{Q}_{e x}$ and $\mathrm{R} / \mathrm{Q}$ for the concerned monopole modes in the third pass band.

\begin{tabular}{cccccc}
\hline \multicolumn{3}{c}{ Symmetrical cavity } & \multicolumn{3}{c}{ Asymmetrical cavity } \\
Frequency & $\mathrm{R} / \mathrm{Q}$ & $\mathrm{Q} e x$ & Frequency & $\mathrm{R} / \mathrm{Q}$ & $\mathrm{Q}_{e x}$ \\
$\left(\mathrm{x} 10^{3} \mathrm{MHz}\right)$ & $\Omega$ & & $\left(\mathrm{x} 10^{3} \mathrm{MHz}\right)$ & $\Omega$ & \\
\hline 2.73730 & $9.47 \mathrm{E}+02$ & $1.44 \mathrm{e}+07$ & 2.73728 & $6.15 \mathrm{E}-01$ & $4.34 \mathrm{e}+05$ \\
2.84779 & $3.08 \mathrm{E}+00$ & $2.85 \mathrm{e}+10$ & 2.84774 & $3.16 \mathrm{E}+00$ & $7.74 \mathrm{e}+06$ \\
2.84790 & $1.20 \mathrm{E}+01$ & $2.48 \mathrm{e}+09$ & 2.84782 & $8.57 \mathrm{E}+00$ & $1.81 \mathrm{e}+07$ \\
2.84808 & $5.02 \mathrm{E}+00$ & $1.45 \mathrm{e}+09$ & 2.84795 & $9.11 \mathrm{E}+00$ & $1.68 \mathrm{e}+07$ \\
2.84834 & $1.45 \mathrm{E}-01$ & $8.37 \mathrm{e}+08$ & 2.84817 & $9.13 \mathrm{E}-01$ & $1.74 \mathrm{e}+07$ \\
2.84869 & $1.20 \mathrm{E}+00$ & $4.26 \mathrm{e}+08$ & 2.84847 & $5.95 \mathrm{E}-01$ & $1.70 \mathrm{e}+07$ \\
2.84910 & $4.16 \mathrm{E}-02$ & $2.07 \mathrm{e}+08$ & 2.84884 & $2.30 \mathrm{E}-01$ & $2.06 \mathrm{e}+07$ \\
2.84955 & $6.29 \mathrm{E}-01$ & $1.77 \mathrm{e}+08$ & 2.84926 & $2.46 \mathrm{E}-01$ & $2.48 \mathrm{e}+07$ \\
2.84994 & $1.18 \mathrm{E}-02$ & $2.04 \mathrm{e}+08$ & 2.84967 & $1.16 \mathrm{E}-01$ & $3.91 \mathrm{e}+07$ \\
2.85045 & $1.85 \mathrm{E}+00$ & $2.91 \mathrm{e}+07$ & 2.85000 & $7.74 \mathrm{E}-02$ & $1.04 \mathrm{e}+08$ \\
2.85046 & $4.04 \mathrm{E}-02$ & $2.49 \mathrm{e}+07$ & 2.85046 & $9.17 \mathrm{E}-01$ & $4.14 \mathrm{e}+07$ \\
\hline
\end{tabular}

It can be noticed that $Q_{e x}$ decreases significantly after optimization of one of end 
cell for asymmetrical cavity. $Q_{e x}$ for HOMs in asymmetrical cavity are placed in range of $10^{6}-10^{8}$ which is appropriate from beam dynamics point of view in SILC section 64]. Comparison between symmetrical and asymmetrical 11-cell cavity for $Q_{e x}$ and impedances $\left(R / Q \cdot Q_{e x}\right)$ of concerned monopole modes in third passband is shown in Figure 3.18 .

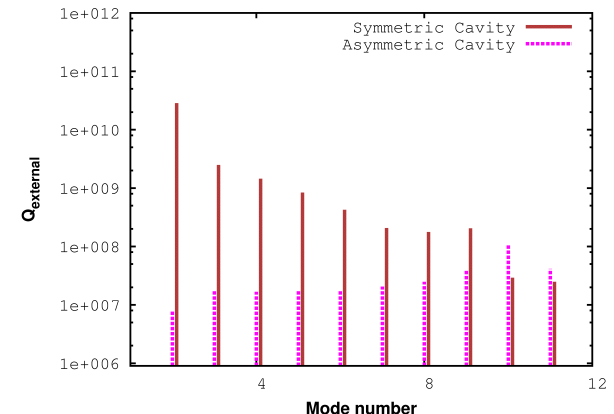

(a)

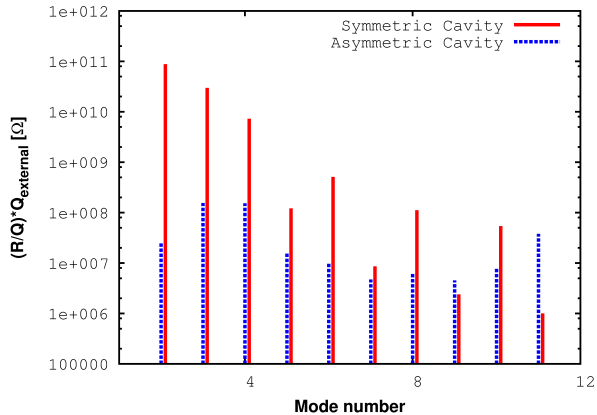

(b)

Figure 3.18: Comparison of symmetrical and asymmetrical 11-cell cavity for (a) $\mathrm{Q}_{e x}$ and (b) impedances $\left(\mathrm{R} / \mathrm{Q} * \mathrm{Q}_{e x}\right)$ of concerned monopole modes in third pass band.

Dipole modes are also investigated and no trapped modes are found. Most concerned dipole modes in first three pass bands are listed in Table 3.7. It is observed that dipole modes have smaller influence of insertion of asymmetry in cavity and $\mathrm{Q}_{e x}$ for dipole mode in asymmetrical cavity are also placed in range of $10^{5}-10^{6}$.

\subsubsection{Design of 9-cell SILC cavity}

Although 11-cell cavity is optimized for SILC section yet there were few concerns from its fabrication point of view. Presence of 11-cell makes it more sensitive to fabrication errors, thus industrial yield is expected to be low. Successful development and fabrication experience of SC $1.3 \mathrm{GHz}$, 9-cell ILC cavity for the TESLA project lead us to design 9-cell, $\beta_{G}=0.81,1.3 \mathrm{GHz}$ cavity as an alternative of 11-cell cavity.

Inner cell design: Length of inner cell will remain same as inner cell in 11-cell but reduction in number of cells for 9-cell cavity will result in decrease in required cell to cell coupling. If we design the 9-cell cavity with same field flatness as in 9-cell ILC cavity expecting that fabrication error will remain same in both cases, the required coupling 


\section{DESIGN OF SCRF CAVITY FOR THE PROJECT-X LINAC}

Table 3.7: $\mathrm{Q}_{e x}$ and $\mathrm{R} / \mathrm{Q}$ for the most concerned dipole modes in the first three pass bands of SILC cavities.

\begin{tabular}{cccccc}
\hline \multicolumn{3}{c}{ Symmetrical cavity } & \multicolumn{3}{c}{ Asymmetrical cavity } \\
$\begin{array}{c}\text { Frequency } \\
(\mathrm{MHz})\end{array}$ & $\mathrm{R} / \mathrm{Q}$ & $\mathrm{Q} e x$ & $\begin{array}{c}\text { Frequency } \\
\mathrm{R} / \mathrm{Q}\end{array}$ & $\mathrm{Q}_{e x}$ \\
\hline 1757.74 & $1.21 \mathrm{e}-06$ & $3.19 \mathrm{e}+06$ & 1757.73 & 0.0004 & $2.11 \mathrm{e}+06$ \\
1758.8 & 0.0634 & $9.39 \mathrm{e}+05$ & 1758.76 & 0.0754 & $6.73 \mathrm{e}+05$ \\
1760.25 & 0.0010 & $5.04 \mathrm{e}+05$ & 1760.17 & 0.0021 & $4.04 \mathrm{e}+05$ \\
1761.78 & 0.4035 & $3.85 \mathrm{e}+05$ & 1761.69 & 0.4521 & $3.42 \mathrm{e}+05$ \\
1763.21 & 0.0276 & $3.58 \mathrm{e}+05$ & 1763.11 & 0.0058 & $3.32 \mathrm{e}+05$ \\
1764.45 & 6.0269 & $3.93 \mathrm{e}+05$ & 1764.36 & 5.5097 & $3.95 \mathrm{e}+05$ \\
1765.47 & 12.607 & $4.68 \mathrm{e}+05$ & 1765.4 & 12.6381 & $4.66 \mathrm{e}+05$ \\
1766.26 & 3.5942 & $7.15 \mathrm{e}+05$ & 1766.21 & 4.04101 & $6.89 \mathrm{e}+05$ \\
1766.81 & 0.0368 & $1.42 \mathrm{e}+06$ & 1766.79 & 0.01029 & $1.35 \mathrm{e}+06$ \\
\hline
\end{tabular}

coefficient is $1.87 \%$ which is same as in TESLA cavity. Since in an elliptical multi-cell cavity cell to cell coupling typically takes place via the electric field through the irises, it is necessary to re-optimize inner celll geometry for new iris radius $\left(R_{\text {iris }}\right)$. Operational requirements of the cavity, defined by the normalized field enhancement factor ratio and field non-uniformity, further fix the values of the other independent geometrical parameters. To compare the field enhancement factor of different geometrical shapes with ILC cavity design, we introduce normalized field enhancement factors, $e$ and $h$, which are defined as:

$$
e=\frac{\left(\frac{E_{p}}{E_{a c c}}\right)_{S I L C}}{\left(\frac{E_{p}}{E_{a c c}}\right)_{I L C}} \text { and } h=\frac{\left(\frac{H_{p}}{E_{a c c}}\right)_{S I L C}}{\left(\frac{H_{p}}{E_{a c c}}\right)_{I L C}}
$$

where values of the electric field enhancement factor $\left(E_{p} / E_{a c c}\right)$ and Magnetic field enhancement factor $\left(\mu_{0} \mathrm{H}_{p} / \mathrm{E}_{a c c}\right)$ for ILC cavity are 2.0 and $4.26 \mathrm{mT} /(\mathrm{MV} /$ meter $)$ respectively [62].

To obtain the same electromagnetic performance and field-flatness as obtained in the ILC cavity, we choose $(h / e) \sim 1$, and $k \sim 1.87 \%$ for 9 -cell. Different cavity shapes are studied for different geometrical parameters. Figure 3.19 shows the result of the tuning procedure, where the normalized field enhancement factors i.e., $e$ vs. $h$ are plotted for five different sets of geometrical parameters $(A, B, a, b)$ and for a given value of $R_{\text {iris }}=$ 


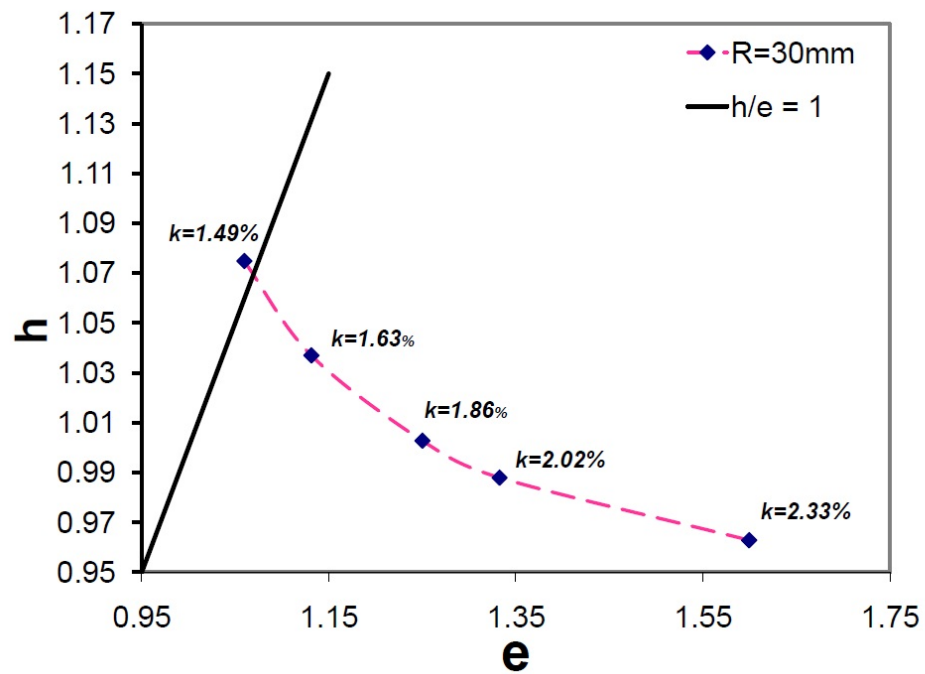

Figure 3.19: Optimization curve for inner cell for $R_{\text {iris }}=30 \mathrm{~mm}$.

$30 \mathrm{~mm}$. For reference, a straight line having $(h / e)=1$ is also shown on the same plot. Cell-to-cell coupling value, which is different for each set, is further mentioned. It can be seen that for $(h / e)=1$, we get a value of $k \sim 1.5 \%$, slightly away from $1.9 \%$. Thus, to meet the requirements of $k, R_{\text {iris }}$ is further varied and tuning results are shown for

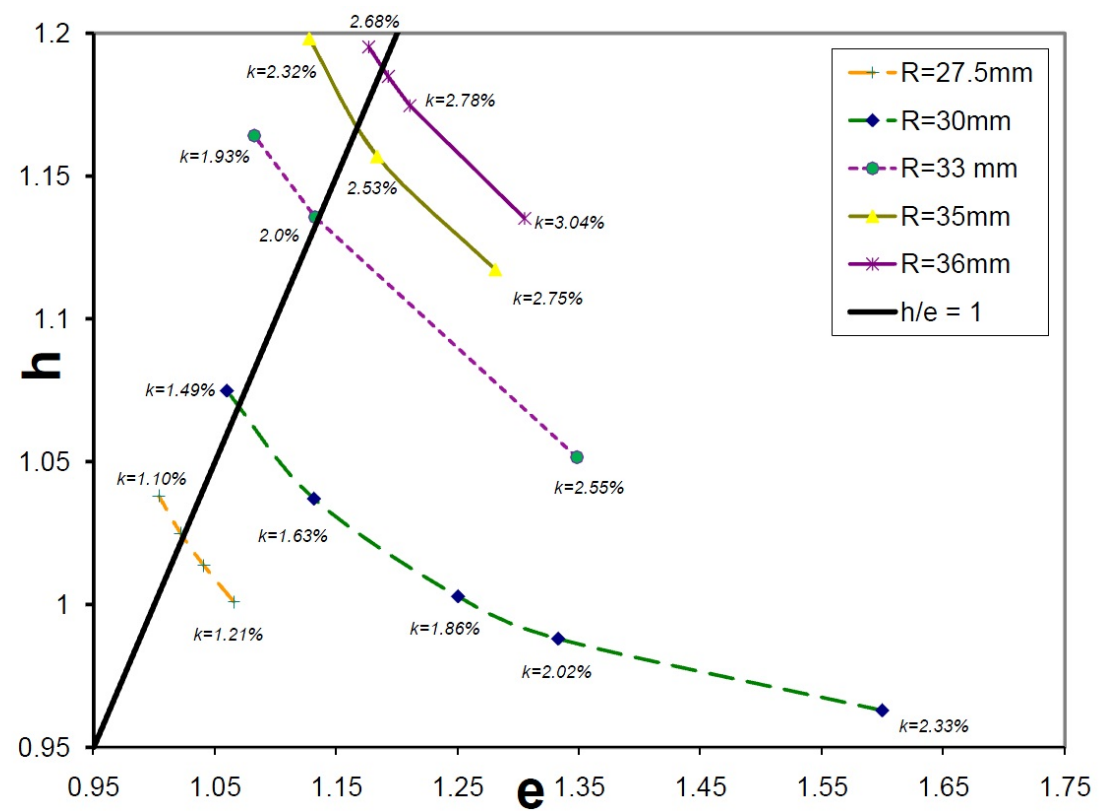

Figure 3.20: Optimization curve for inner cell for various values of $\mathrm{R}_{\text {iris }}$. 


\section{DESIGN OF SCRF CAVITY FOR THE PROJECT-X LINAC}

various sets of geometrical parameters in Figure 3.20 . It is found that for $\mathrm{R}_{\text {iris }}=33$ $\mathrm{mm}$, we get $(h / e)=1$ and $k \sim 2 \%$, close to our operational requirements.

Design of End cell: After designing inner cell, outer half of end cell is optimized in order to achieve good field flatness. It also takes into account that natural eigen frequency spectrum of HOMs should not deviate too much for inner cell and end cell. Field enhancement factors for end cells are kept less than the inner cell.

Geometrical and RF parameters: A symmetric 9-cell, $\beta_{G}=0.81,1.3 \mathrm{GHz}$ cavity is designed. Geometrical representation of half inner and half end cell are shown in Figure 3.21 while magnitudes of corresponding geometrical parameters are summarized in Table 3.8.

Table 3.8: Geometrical parameters for inner and end cell of 9-cell $\beta_{G}=0.811 .3 \mathrm{GHz}$ cavity.

\begin{tabular}{cccc}
\hline Parameters & Unit & Half inner cell & Half end cell \\
\hline $\mathrm{R}_{\text {iris }}$ & $\mathrm{mm}$ & 33 & 39 \\
$\mathrm{R}_{\text {eq }}$ & $\mathrm{mm}$ & 102.56 & 102.56 \\
$\mathrm{~A}$ & $\mathrm{~mm}$ & 33.0 & 33.5 \\
$\mathrm{~B}$ & $\mathrm{~mm}$ & 35.0 & 35.0 \\
$\mathrm{a}$ & $\mathrm{mm}$ & 12.0 & 10.0 \\
$\mathrm{~b}$ & $\mathrm{~mm}$ & 21.0 & 20.0 \\
$\mathrm{~L}$ & $\mathrm{~mm}$ & 46.752 & 44.63 \\
$\alpha$ & degree & 5.8 & 5.3 \\
\hline
\end{tabular}

From the electric field distribution of operating mode (Figure 3.22a), field flatness is found to be uniform $(2.24 \%)$. The dispersion curve is shown in Figure $3.22 \mathrm{~b}$ for first monopole pass band which consists of the fundamental mode. Positive slope of dispersion curve suggests that cells are coupled electrically. The relative separation of fundamental mode with its neighbour is $0.24 \%$ which is larger than ILC RF cavity $(0.064 \%)$ and 11-cell RF cavity (0.054 \%). This implies looser tolerances on tuning of RF cavity.

RF parameters for operating modes are studied. It is found that cavity achieves its optimal performance for particle traveling with $84 \%$ of velocity of light. Results are summarized for both geometrical beta $\left(\beta_{G}=0.81\right)$ and optimal beta $\left(\beta_{\text {opt }}=0.84\right)$ in Table $(3.9)$. 


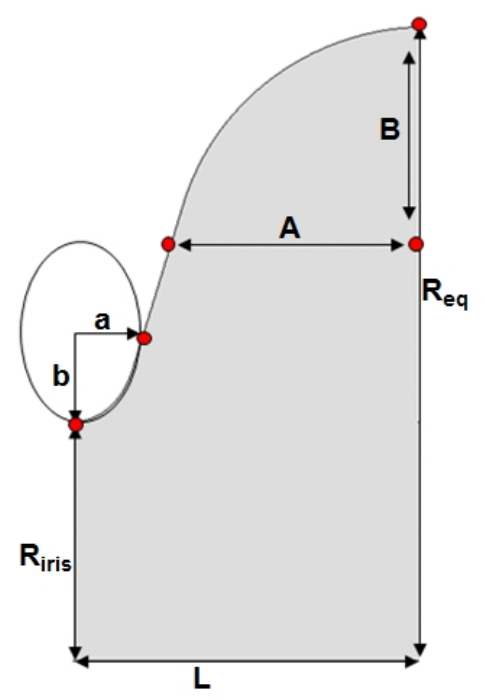

Half Inner cell

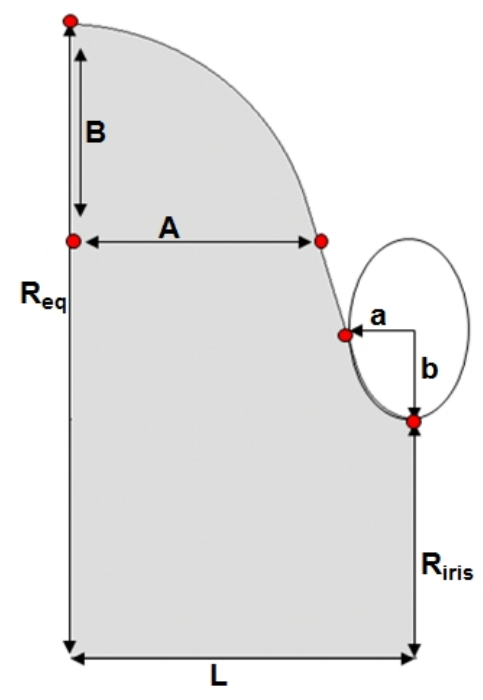

Half End cell

Figure 3.21: half inner cell (left) and half end cell (right).

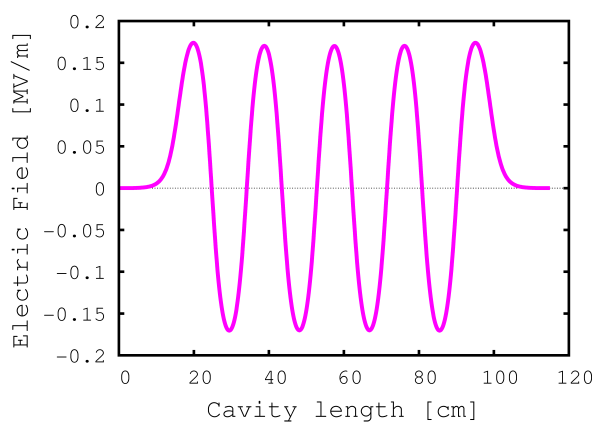

(a)

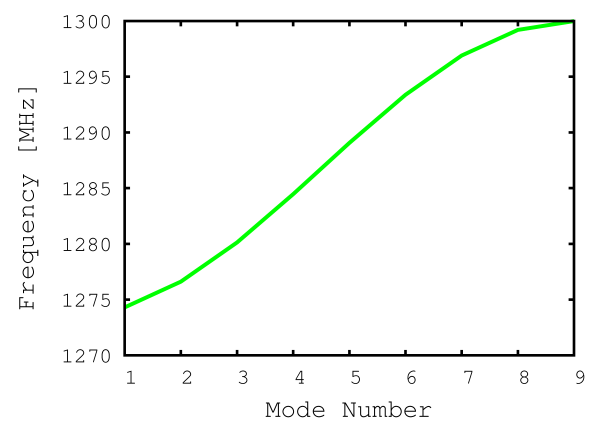

(b)

Figure 3.22: 9 cell cavity - (a) Electric field distribution and (b) dispersion curve for first monopole pass band which consists of the fundamental mode.

HOMs study : 9-cell RF cavity has been studied for monopole (longitudinal) and dipole (transverse) HOMs. Investigation has been performed for potentially trapped modes. The distribution of effective impedance of longitudinal and transverse HOM are shown in Figure 3.23a and Figure 3.23b respectively for $\beta_{G}=0.81$. The effective 
Table 3.9: RF Parameters for fundamental mode of 9-cell $\beta_{G}=0.811 .3 \mathrm{GHz}$ cavity.

\begin{tabular}{cccc}
\hline Parameters & Unit & \multicolumn{2}{c}{ Magnitude } \\
\hline & & $\beta_{G}=0.81$ & $\beta_{\text {opt }}=0.84$ \\
Transit time factor & - & 0.77 & 0.79 \\
Coupling coefficient & $\%$ & 2 & 2 \\
$E_{p} / E_{a c c}$ & - & 2.26 & 2.24 \\
$B_{p} / E_{a c c}$ & $\mathrm{mT} /(\mathrm{MV} / \mathrm{m})$ & 4.77 & 4.72 \\
$\mathrm{R} / \mathrm{Q}$ & $\Omega$ & 677 & 691 \\
$\mathrm{G}$ & $\Omega$ & 226 & 226 \\
Frequency & $\mathrm{GHz}$ & 1.3 & 1.3 \\
\hline
\end{tabular}

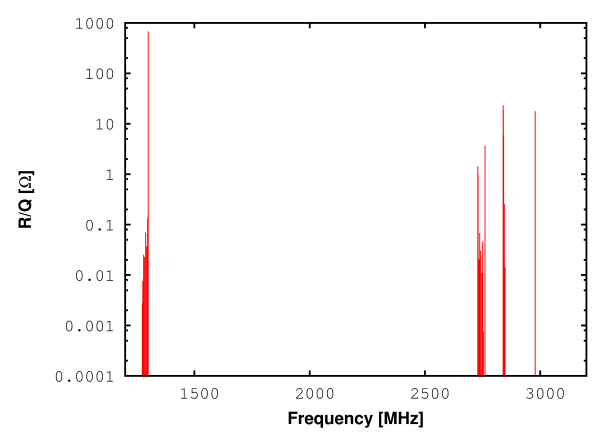

(a)

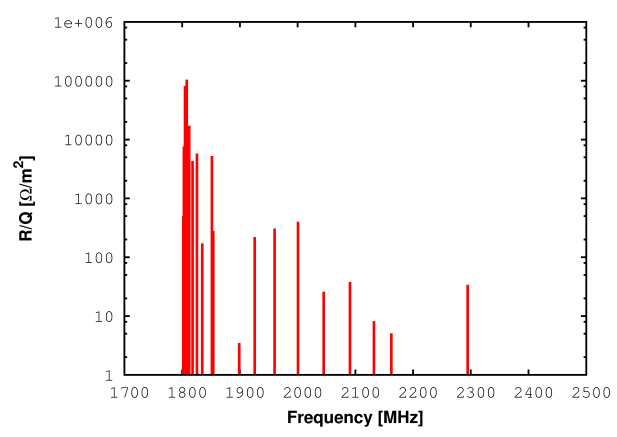

(b)

Figure 3.23: HOMs spectrum for 9 cell-cavity - Impedance of (a) monopole modes $\mathrm{R} / \mathrm{Q}$ and $(\mathrm{b})$ Transverse modes $(R / Q)_{\|}$are shown for $\beta_{G}=0.81$.

impedance is less than $25 \Omega$ for the most concerned longitudinal mode and less than 10 $\Omega / \mathrm{cm}^{2}$ for transverse modes. Beta dependence of the some monopole HOMs with high effective impedance is shown in Figure 3.24 .

The monopole modes corresponding to frequencies $2838.97 \mathrm{MHz}$ and $2840.12 \mathrm{MHz}$ have highest effective impedances. The mode for the frequency of $2838.97 \mathrm{MHz}$ has highest effective impedance of $28 \Omega$ for $\beta=0.83$ and mode corresponding to the frequency $2840.12 \mathrm{MHz}$ has the same impedance for $\beta=0.90$. To investigate the nature of both modes, electric field distribution are illustrated in Figure 3.25. It can be seen that these modes are not trapped because they have sufficient amplitude within at least one of end cells to ensure their effective coupling with HOM coupler. 


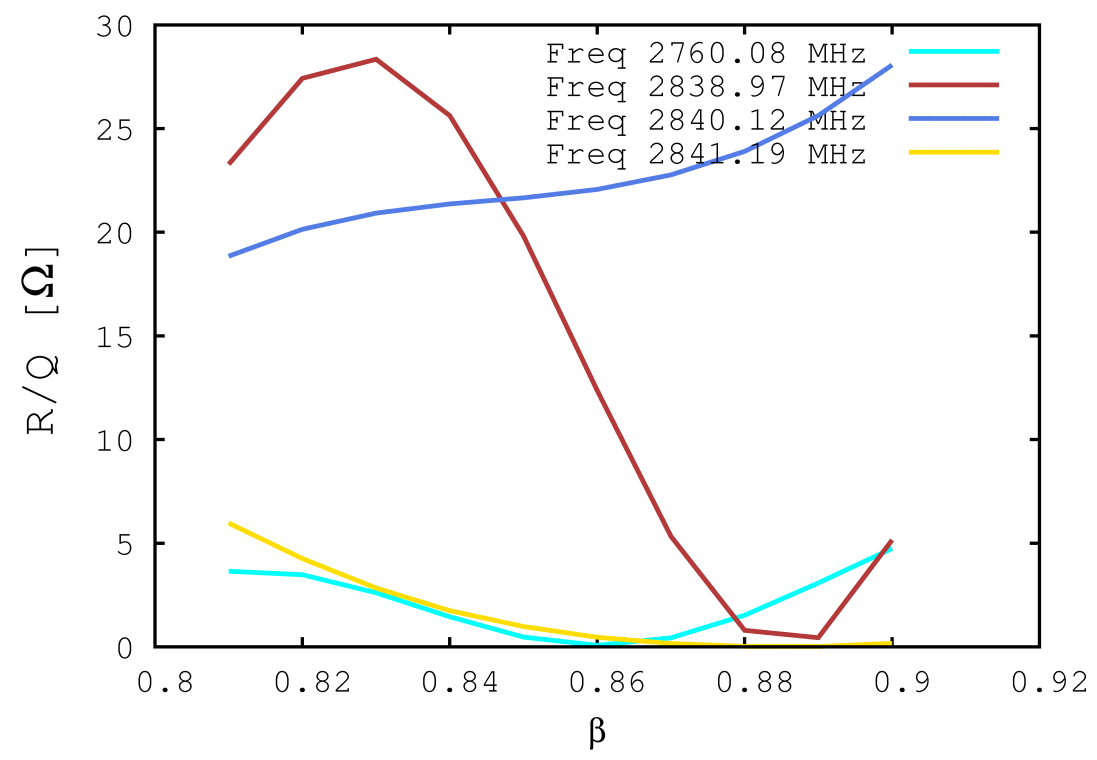

Figure 3.24: Beta dependence of the monopole HOM with high effective impedance.

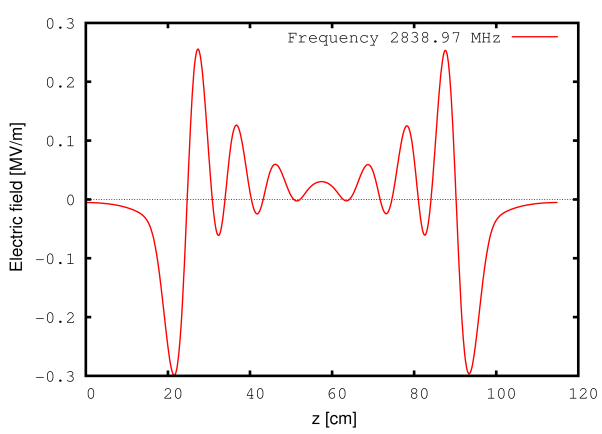

(a)

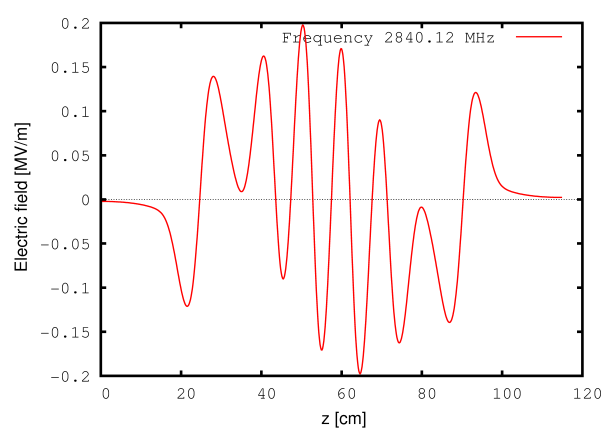

(b)

Figure 3.25: Electric field distribution for the most concerned monopole modes - with frequency of (a) $2838.97 \mathrm{MHz}$ and (b) $2840.12 \mathrm{MHz}$ for SILC 9-cell cavity. 


\section{DESIGN OF SCRF CAVITY FOR THE PROJECT-X LINAC}

\subsection{Design of SCRF cavity for the CW linac}

\subsubsection{Why transition from $325 \mathrm{MHz}$ to $650 \mathrm{MHz}$ frequency instead of $1.3 \mathrm{GHz}$ ?}

Two families of SC elliptical cavities are designed for the acceleration of particles traveling with $61 \%$ and $90 \%$ of speed of light. These are operated at $650 \mathrm{MHz}$ and used for acceleration of beam from $160 \mathrm{MeV}$ to $3 \mathrm{GeV}$ in $\mathrm{CW}$ linac. Operation at $650 \mathrm{MHz}$ instead of $1.3 \mathrm{GHz}$ is preferred due to following reasons:

\section{- Beam dynamics prospects:}

- Choice of operation of cavity at $650 \mathrm{MHz}$ simplifies the beam dynamics. Project-X front end operates at $325 \mathrm{MHz}$, and 2-fold frequency jump at transition to the higher energy stage for $650 \mathrm{MHz}$ is easier than 4-fold for 1.3 GHz. Thus, It enhances RF bucket area available for particles in longitudinal phase space (energy and time).

- Transverse size of cavity scales inversely with frequency. Thus, lower frequency means large transverse aperture which results in increase in phase space area available in transverse planes (horizontal and vertical plane).

- Cavity not only provides accelerating kick to the beam but it also gives transverse kick. It is inversely proportional to operating frequency of cavity. Transverse kick can degrade beam quality particularly at low energy. Thus, lowering in frequency also reduces transverse kick by the cavity which helps in maintaining the beam quality along the linac.

- HOM impedances (transverse and longitudinal) are smaller at lower frequency, and it may in principle allow one to get rid of HOM dampers, which may be a source of many problems for proton accelerators such as multipacting, RF leak etc. It is convenient to assemble a cavity without HOM damper.

- Losses caused by intra-beam stripping will be smaller for lower frequency as well.

- Lower operating frequency of cavity leads to better cavity and beam interaction. Figure 3.26 shows energy gain of $650 \mathrm{MHz}$ and $1300 \mathrm{MHz}$ cavities for the energy 
range of $500 \mathrm{MeV}$ to $3 \mathrm{GeV}$. Two families of cavities are used for each frequency. The RF load (for two families of cavities) for $1300 \mathrm{MHz}$ are $20.5 \mathrm{~W}$ and $17.3 \mathrm{~W}$, whereas it is $18.1 \mathrm{~W}$ and $18.5 \mathrm{~W}$ for $650 \mathrm{MHz}$ cavities. The gradients in cavities are selected for peak surface magnetic field of $72 \mathrm{~T}$. It can be noticed that energy gain is higher for $650 \mathrm{MHz}$ cavities. It can be easily understood from equation (3.4) which suggest that for same geometrical beta $\left(\beta_{G}\right)$, energy gain is inversely proportional to frequency.

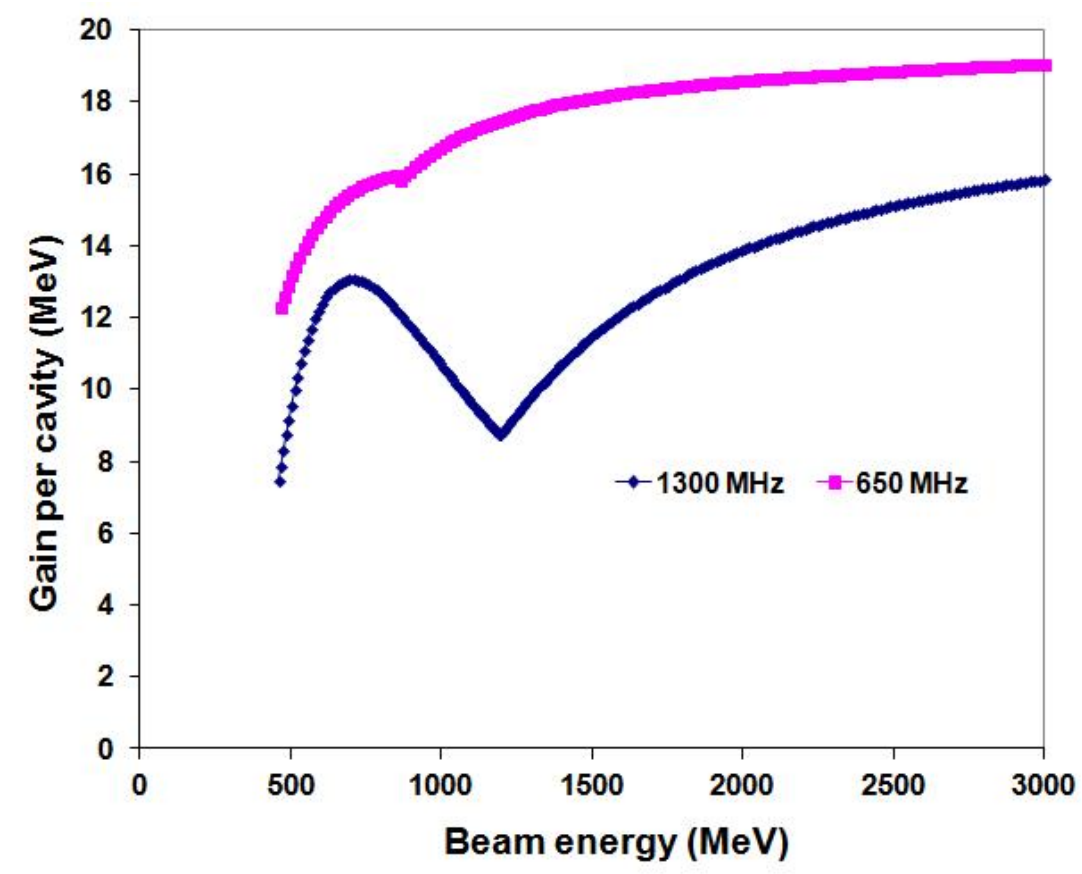

Figure 3.26: Comparison of acceleration efficiencies of $1300 \mathrm{MHz}$ and $650 \mathrm{MHz}$ cavities.

- Length of 5-cell, $\beta_{G}=0.90$ cavity is approximately same as that of 9-cell, $\beta_{G}=1$, $1.3 \mathrm{GHz}$ cavity. Thus maximum energy gain per cavity is same in both cases and power requirement is also same.

Concept of $650 \mathrm{MHz}$ frequency is similar as SNS, SPL and ESS cavities. With lot of advantages, there are some trade-off of lower frequency application as well. These are summarized as following:

- Cavities for $650 \mathrm{MHz}$ are bigger in size and thus more $\mathrm{Nb}$ is required to build a cavity which makes it more expensive than $1.3 \mathrm{GHz}$ cavities. This increase in 


\section{DESIGN OF SCRF CAVITY FOR THE PROJECT-X LINAC}

price can be compensated by smaller number of cavities and RF sources. Higher acceleration efficiency leads to lower number of cavties for the given accelerating range. Avoiding $\mathrm{HOM}$ damper for $650 \mathrm{MHz}$ cavities further reduces cost of dressed cavity (cavity and all auxiliary components).

- It is required to rebuild infrastructure in Fermilab to handle $650 \mathrm{MHz}$ cavities. Mechanical tuning machine, facilities for surface treatment such as furnace size and acid container for electro polishing, vertical test stand etc. which should be capable to support $650 \mathrm{MHz}$ cavities.

\subsubsection{Design of medium beta cavity $\left(\beta_{G}=0.61\right)$ for intermediate en- ergy section of linac}

Generally medium beta cavity are not designed to operate at very high accelerating gradient. $\beta_{G}=0.61,5$-cell cavity is designed with considerations of all the requirements of intermediate energy section of CW linac of Project-X. These are operated at accelerating gradient in the energy range of 7 to $14 \mathrm{MV} / \mathrm{m}$.

Inner cell design: It can be noticed from Figure 3.27 that field enhancement

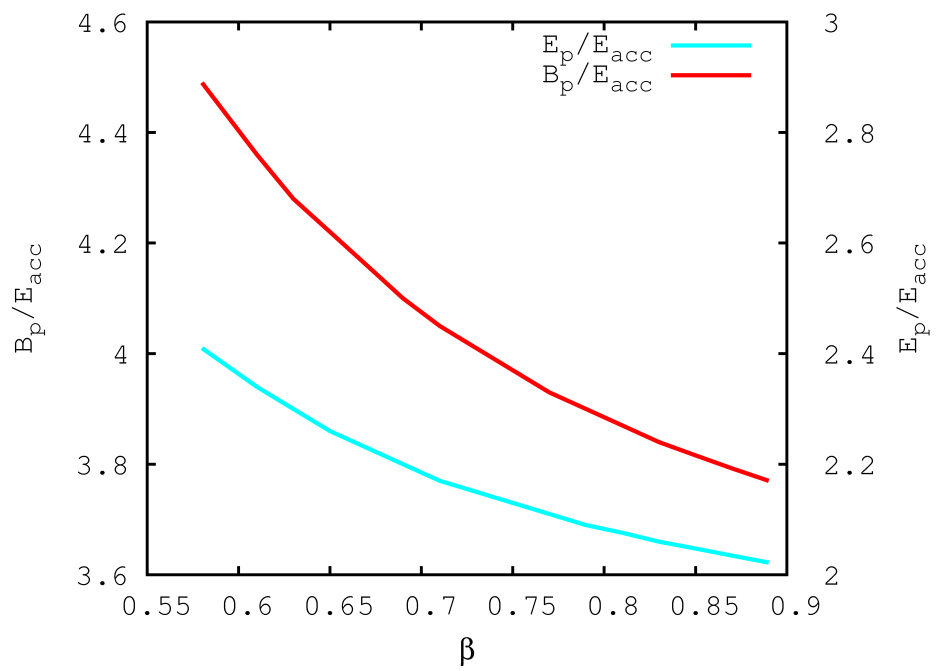

Figure 3.27: Variation of electric and magnetic field enhancement factor with relative particle velocity $(\beta)$. 
factors increase with decrease in $\beta$. Thus, the goal of the cavity shape optimization was again to reduce the field enhancement factors to achieve maximum possible gradient. In order to do this iris aperture is kept small and chosen to $43 \mathrm{~mm}$ which is large enough to provide $k$ greater than $0.6 \%$ for fundamental pass band. Small cavity wall inclination angle also provides more freedom to achieve lower field enahancement factors. It is reduced to $2^{\circ}$ for present design. Iris aperture and wall inclination angle are biggest concern from surface processing point of view but these values for $\beta_{G}=0.61$ cavity seems feasible.

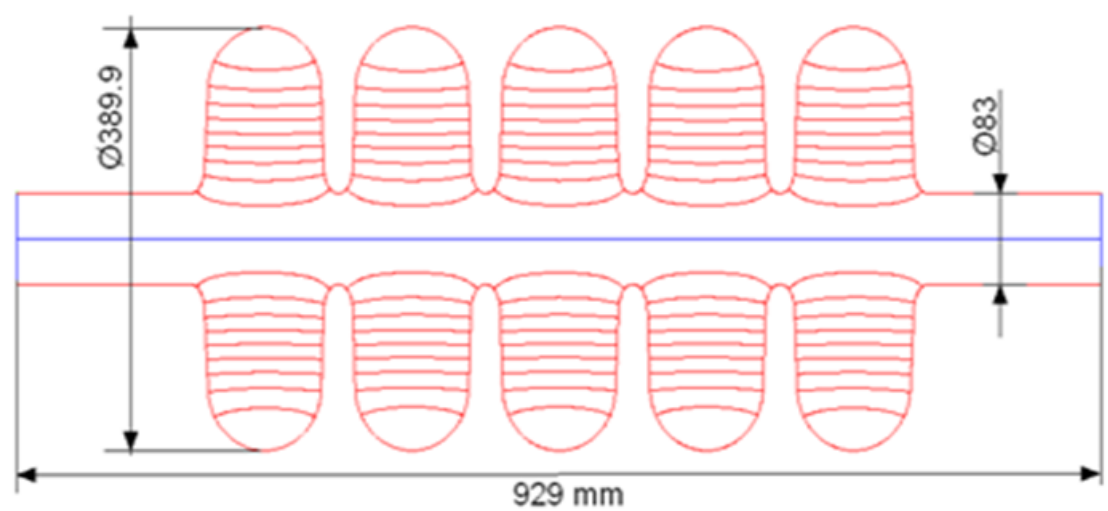

Figure 3.28: $650 \mathrm{MHz}, 5$-cell, $\beta_{G}=0.61$ cavity.

Table 3.10: Geometrical parameters for half inner and half end cell of 5-cell, $\beta_{G}=0.61$, $650 \mathrm{MHz}$ cavity.

\begin{tabular}{cccc}
\hline Parameters & Unit & Half inner cell & Half end cell \\
\hline $\mathrm{R}_{\text {iris }}$ & $\mathrm{mm}$ & 41.5 & 41.5 \\
$\mathrm{R}_{e q}$ & $\mathrm{~mm}$ & 194.952 & 194.952 \\
$\mathrm{~A}$ & $\mathrm{~mm}$ & 54.0 & 54.0 \\
$\mathrm{~B}$ & $\mathrm{~mm}$ & 58.0 & 58.0 \\
$\mathrm{a}$ & $\mathrm{mm}$ & 14.0 & 14.0 \\
$\mathrm{~b}$ & $\mathrm{~mm}$ & 25.0 & 25.0 \\
$\mathrm{~L}$ & $\mathrm{~mm}$ & 70.34 & 71.385 \\
$\alpha$ & degree & 1.86 & 2.7 \\
\hline
\end{tabular}

End cell design: $\beta_{G}=0.61$, cavity is symmetrical cavity. End cells are optimized 


\section{DESIGN OF SCRF CAVITY FOR THE PROJECT-X LINAC}

in order to achieve same operating frequency as inner cell and attaining good field flatness. Field enhancement factors of end cells are kept below the inner cell. Figure 3.28 shows schematic of 5-cell, $650 \mathrm{MHz}$ cavity. Geometrical parameters for optimized shape of inner cell and end cells are listed in Table 3.10. It should be noted that cavity shape is optimized at operating temperature. Thus, these are usually called cold dimensions or operating dimensions of cavity. However fabrication dimension of cavity are different from cold dimensions. It includes thermal expansion of material during transition of cold temperature $(2 \mathrm{~K})$ to room temperature $(300 \mathrm{~K})$ and material removal during surface processing of cavity such as electro polishing (EP) and buffer chemical polishing $(\mathrm{BCP})$. It is necessary to account this material removal during cavity fabrication otherwise cavity shape will differ from optimized shape at operating temperature which results in change in operating frequency.

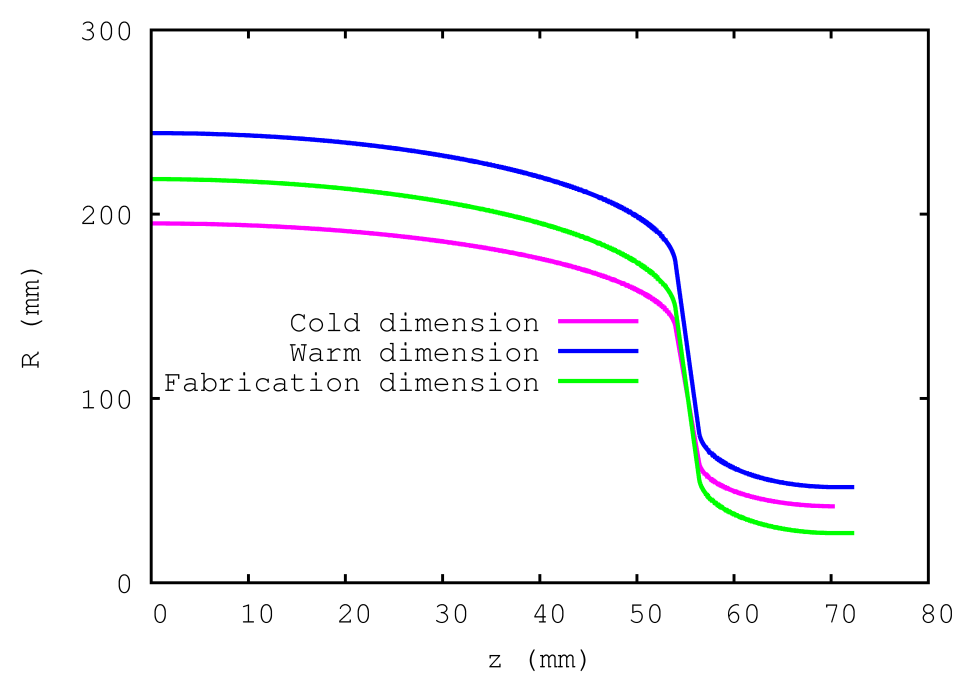

Figure 3.29: Cavity operational and fabrication dimensions.

Figure 3.29 shows schematic of cavity shape for different dimensions at various stages. It can be noticed that material removal results in increase in radius of cavity and semi-axes of equatorial ellipse while it reduces $\mathrm{R}_{\text {iris }}$ and semi axes of iris ellipse. However length of cavity does not change with material removal. Dimensions of half inner and half end cell of $\beta_{G}=0.61$, cavity are summarized in Table 3.11. Warm 
dimensions (room temperature) are calculated by multiplying cold dimensions with scaling factor for $\mathrm{Nb}$ for the transition from $2 \mathrm{~K}$ to $300 \mathrm{~K}$. Fabrication dimensions are obtained by adjusting warm dimension with respect to material removal. It should be noticed that uniform material removal of $150 \mu \mathrm{m}$ is considered for rough approximation. However, material removal at iris and equator of cavity is not uniform in reality.

Table 3.11: Geometrical parameter for half cell of 5 -cell, $\beta_{G}=0.61,650 \mathrm{MHz}$ cavity at different stages.

\begin{tabular}{ccccc}
\hline Parameters & Unit & $\begin{array}{c}\text { Cold } \\
\text { dimensions }\end{array}$ & $\begin{array}{c}\text { Warm } \\
\text { dimensions }\end{array}$ & $\begin{array}{c}\text { Fabrication } \\
\text { dimensions }\end{array}$ \\
\hline $\mathrm{R}_{\text {iris }}$ & $\mathrm{mm}$ & 41.5 & 41.561 & 41.41 \\
$\mathrm{R}_{e q}$ & $\mathrm{~mm}$ & 194.952 & 194.241 & 195.091 \\
$\mathrm{~A}$ & $\mathrm{~mm}$ & 54.0 & 54.08 & 53.93 \\
$\mathrm{~B}$ & $\mathrm{~mm}$ & 58.0 & 58.086 & 57.936 \\
$\mathrm{a}$ & $\mathrm{mm}$ & 14 & 14.021 & 14.171 \\
$\mathrm{~b}$ & $\mathrm{~mm}$ & 25.0 & 25.037 & 25.187 \\
$\mathrm{~L}$ & $\mathrm{~mm}$ & 70.34 & 70.44 & 70.44 \\
$\mathrm{~L}_{\text {end }}$ & $\mathrm{mm}$ & 71.385 & 71.491 & 71.491 \\
\hline
\end{tabular}

RF parameters and field flatness of cavity is calculated for fundamental mode and summarized in Table 3.12. Field non uniformity between cells are found to be $1.3 \%$. Electric field distribution for fundamental mode in 5-cell cavity is shown in Figure 3.30. It may be noted that field is normalized for $1 \mathrm{~mJ}$ stored energy in cavity.

Table 3.12: RF Parameters for fundamental mode of 5 -cell $\beta_{G}=0.61,650 \mathrm{MHz}$ cavity.

\begin{tabular}{cccc}
\hline Parameters & Unit & \multicolumn{2}{c}{ Magnitude } \\
\hline Coupling coefficient & $\%$ & 0.67 & 0.67 \\
$E_{p} / E_{a c c}$ & - & 2.34 & 2.26 \\
$B_{p} / E_{a c c}$ & $\mathrm{mT} /(\mathrm{MV} / \mathrm{m})$ & 4.37 & 4.22 \\
$\mathrm{R} / \mathrm{Q}$ & $\Omega$ & 352 & 367 \\
$\mathrm{G}$ & $\Omega$ & 191 & 191 \\
Frequency & $\mathrm{GHz}$ & 650 & 650 \\
Field flatness & $\%$ & 1.3 & 1.3 \\
Active length & $\mathrm{mm}$ & 705.5 & 705.5 \\
\hline
\end{tabular}




\section{DESIGN OF SCRF CAVITY FOR THE PROJECT-X LINAC}

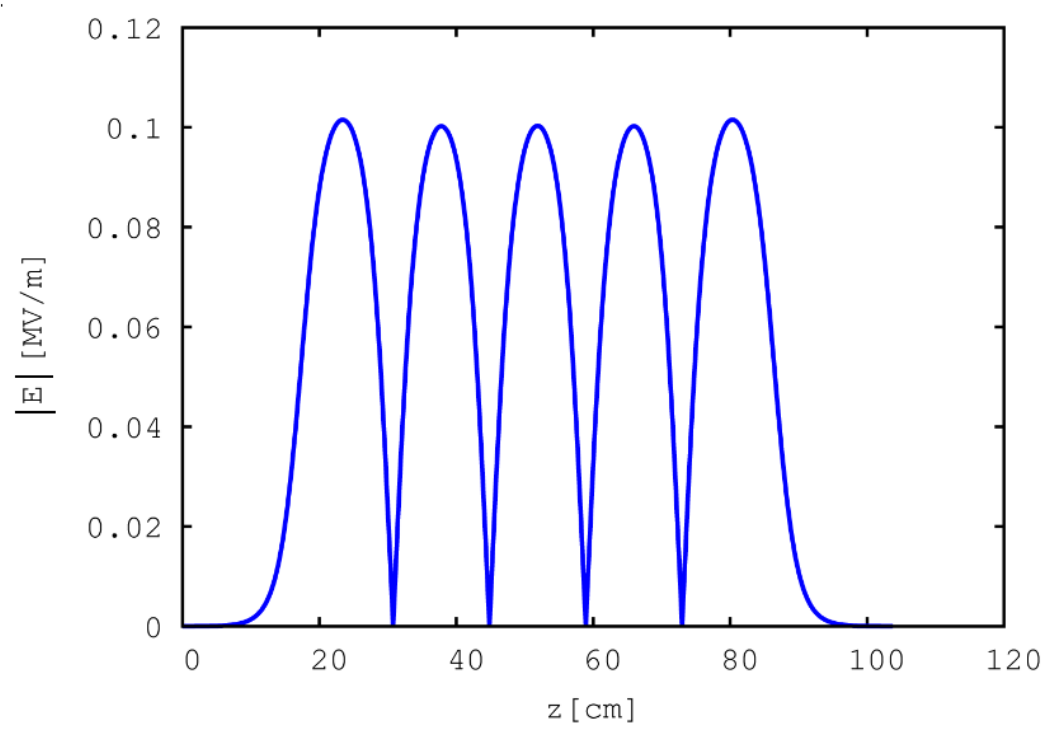

Figure 3.30: Field distribution of fundamental mode in 5-cell $\beta_{G}=0.61650 \mathrm{MHz}$ cavity.

Cavity is also studied for longitudinal and transverse HOMs. Figures 3.31a and $3.31 b$ show monopole and dipole HOM spectrum below the cut-off frequency for $\beta_{G}=$ 0.61. It should be noted that cut off frequency for monopole and dipole mode are 2.76 $\mathrm{GHz}$ and $1.76 \mathrm{GHz}$ respectively. It can be observed from Figure 3.31a that fundamental mode has highest effective imepdance of $352 \Omega$ and next highest effective is below 10 $\Omega$. Investigation is also performed to search for potential trapped modes. It is found that there is no trapped mode in longitudinal and transverse pass bands.

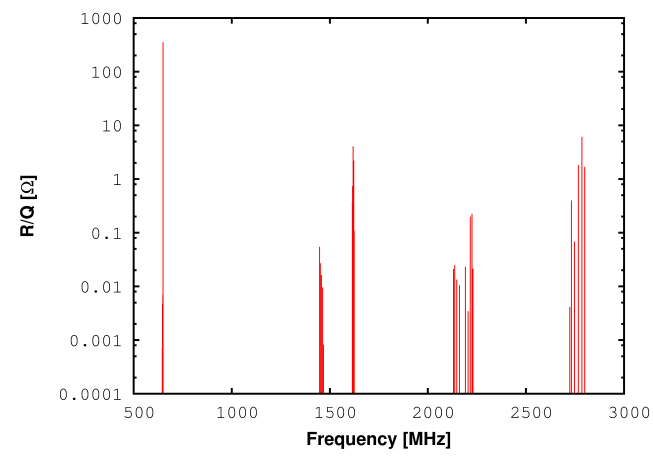

(a)

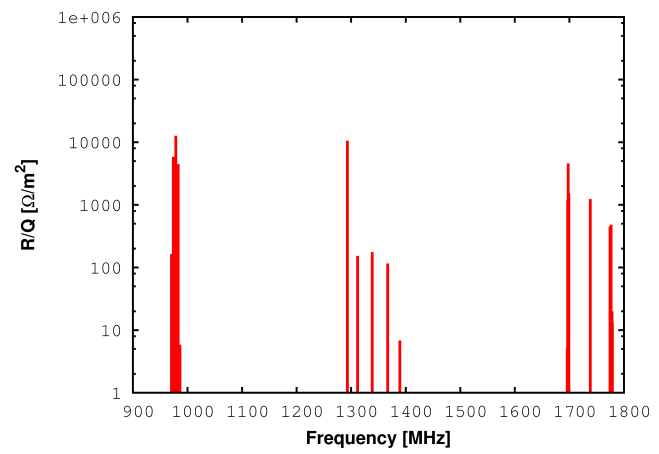

(b)

Figure 3.31: (a)Distribution of effective impedance $\mathrm{R} / \mathrm{Q}(\Omega)$ of longitudinal modes (monopole modes) and (b) transverse effective impedance $(R / Q)_{\|}\left(\Omega / \mathrm{cm}^{2}\right)$ of transverse modes (dipoles) for $\beta_{G}=0.61$. 
Dispersion curves are plotted for first six monopole pass bands (Figure 3.32) and dipole pass bands (Figure 3.33). Dispersion curve represents distribution of normal modes in pass band and provides information about nature as well as strength of coupling between the cells. If cells are connected electrically, $\pi$ mode has highest frequency while it has lowest frequency for magnetically coupled cavity.

It can be noticed from Figure 3.32a that relative separation of operating mode with respect to its neighbouring mode is $0.072 \%$ which is greater than that of the 9-cell ILC cavity (0.064\%). Large separation of operating mode with respect to neighbouring mode increases tolerances of cavity against fabrication errors and also implies easy tunning of cavity.

\subsubsection{Design of high beta cavity $\left(\beta_{G}=0.90\right)$ for high energy section of linac}

$\beta_{G}=0.90,650 \mathrm{MHz}$ elliptical shape SCRF cavity is designed to accelerate the beam from $\sim 500 \mathrm{MeV}$ to $3 \mathrm{GeV}$ in high energy section of $\mathrm{CW}$ linac. It consists of 5-cells which makes its length about the same as $\beta_{G}=1$, ILC 9-cell, $1.3 \mathrm{GHz}$ cavity. Thus maximum energy gain per cavity is also same but with large operating range due to smaller number of cells with respect to ILC cavity. It operates in beam transport line at accelerating gradient in the range of $8 \mathrm{MV} / \mathrm{m}$ to $17 \mathrm{MV} / \mathrm{m}$.

Inner cell design: Cavity shape is optimized in order to minimize field enhancement factors for better interaction between fields and cavity. $\beta_{G}=0.90$ cavity is designed for $\mathrm{R}_{\text {iris }}$ of $50 \mathrm{~mm}$. Inter cell to cell coupling for given iris aperture is $\sim 0.75 \%$. It is large enough to provide same field flatness as in ILC cavity if one assumes same order of fabrication errors in both cavity. Wall inclination angle for optimized shape is $5.2^{\circ}$ which is large enough for surface cleaning and machanical stability point of view.

End cell design: After inner cell optimization, end cells are designed in order to achieve good field flatness and to avoid any potential trapped modes in cavity. Cavity is designed with symmetrical ends. Wall inclination angle for end cells is $7^{\circ}$ which is greater than inner cells. It is convenient to have large wall angle for water and chemical rinsing. Figure 3.34 shows schematic of 5-cell, $\beta_{G}=0.90,650 \mathrm{MHz}$ cavity. Geometrical parameters for optimized shape (cold dimensions) along with warm dimensions and fabrication dimensions are summarized in Table 3.13 . End cell has same $R_{i r i s}, R_{e q}$ and $A$ as inner cell. 


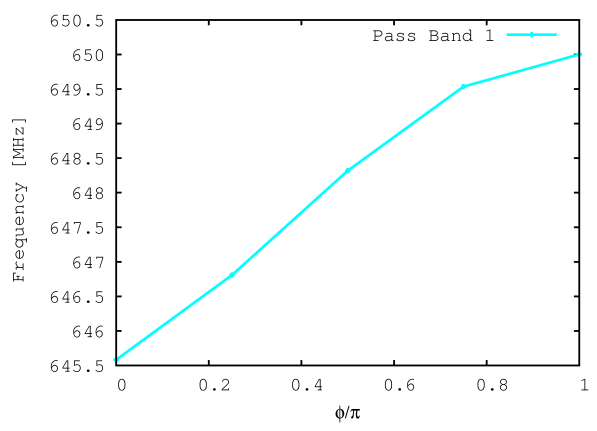

(a)

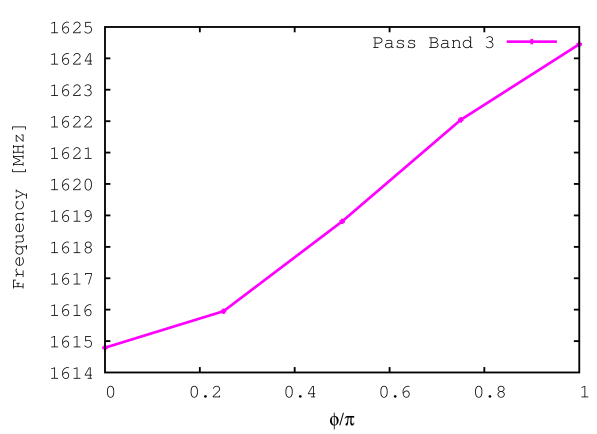

(c)

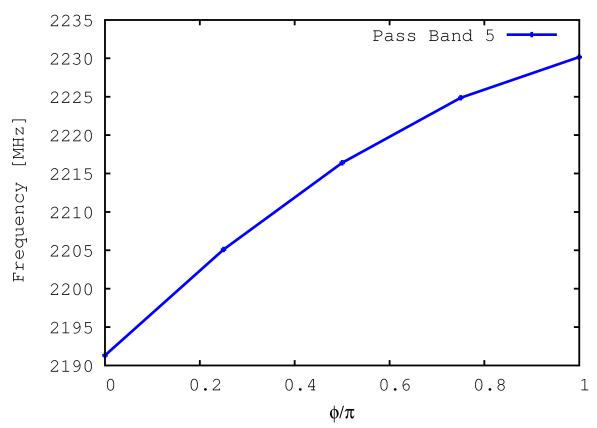

(e)

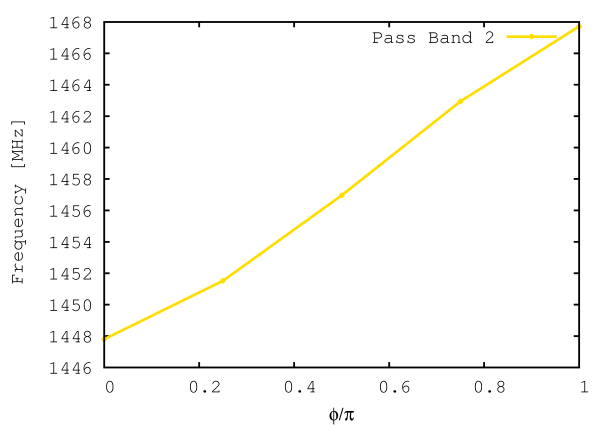

(b)

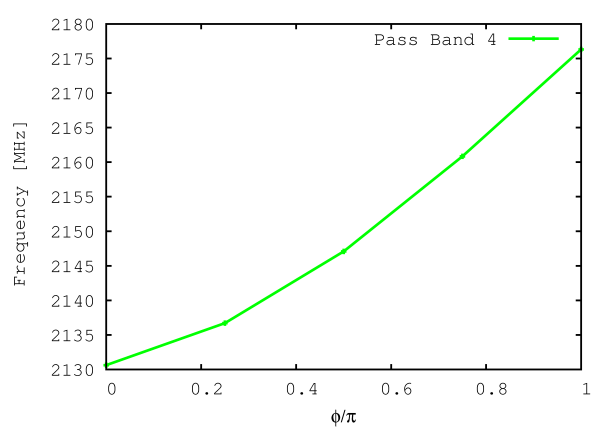

(d)

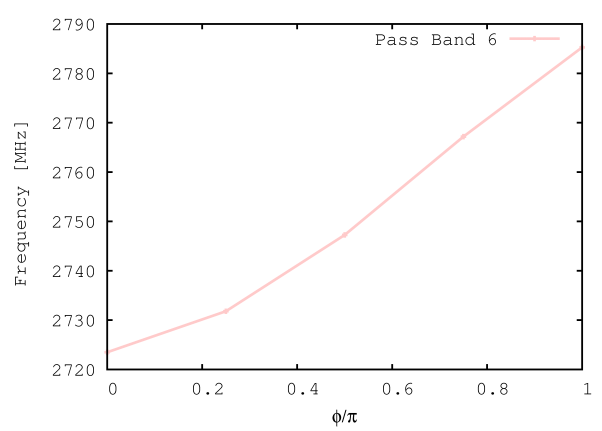

(f)

Figure 3.32: Distribution of monopole modes (longitudinal modes) with phase advance for first six pass bands of 5 -cell, $\beta=0.61,650 \mathrm{MHz}$ cavity. 


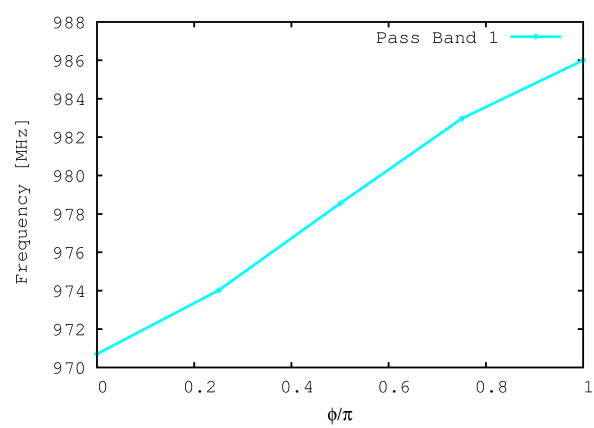

(a)

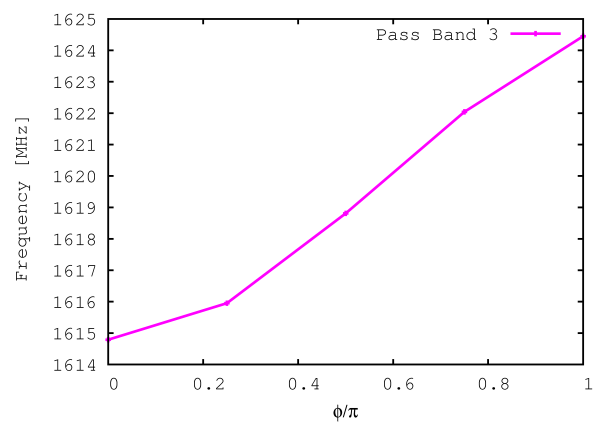

(c)

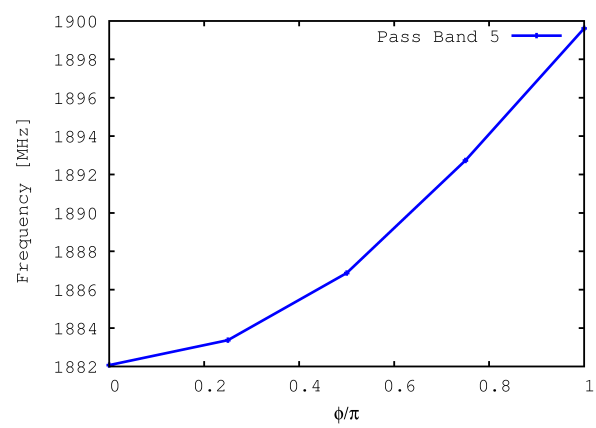

(e)

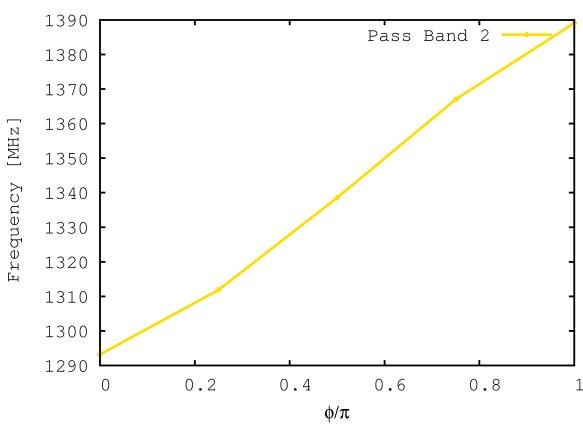

(b)

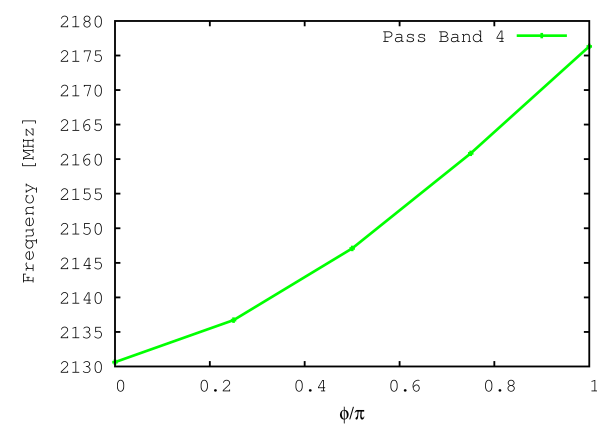

(d)

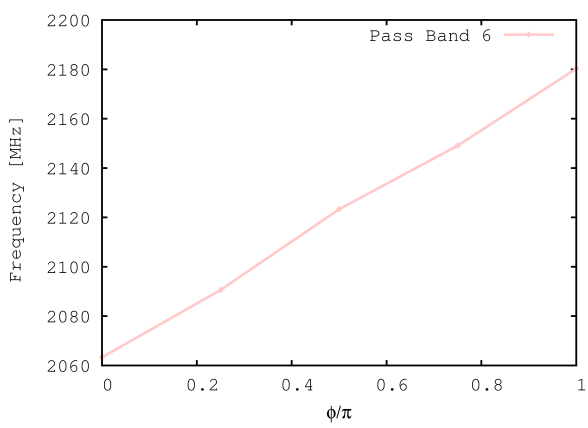

(f)

Figure 3.33: Distribution of dipole modes (transverse modes) with phase advance for first six pass band of 5-cell, $\beta_{G}=0.61,650 \mathrm{MHz}$ cavity. 


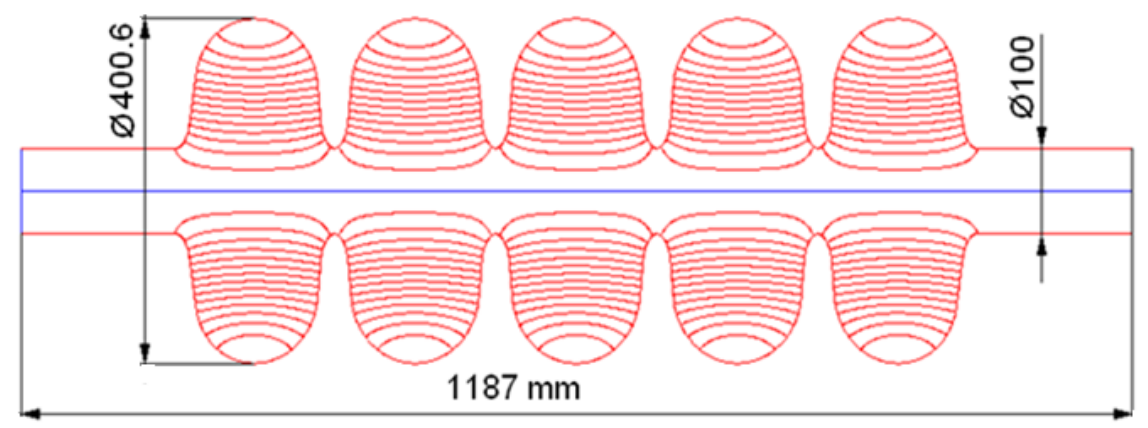

Figure 3.34: 5 -cell, $\beta_{G}=0.90,650 \mathrm{MHz}$ cavity.

Table 3.13: Geometrical parameter for half cell of 5-cell, $\beta_{G}=0.90,650 \mathrm{MHz}$ cavity at different stages.

\begin{tabular}{ccccc}
\hline Parameters & Unit & $\begin{array}{c}\text { Cold } \\
\text { dimensions }\end{array}$ & $\begin{array}{c}\text { Warm } \\
\text { dimensions }\end{array}$ & $\begin{array}{c}\text { Fabrication } \\
\text { dimensions }\end{array}$ \\
\hline $\mathrm{R}_{\text {iris }}$ & $\mathrm{mm}$ & 50.0 & 50.074 & 49.924 \\
$\mathrm{R}_{\text {eq }}$ & $\mathrm{mm}$ & 200.277 & 200.574 & 200.424 \\
$\mathrm{~A}$ & $\mathrm{~mm}$ & 82.5 & 82.622 & 82.472 \\
$\mathrm{~B}$ & $\mathrm{~mm}$ & 84.0 & 84.124 & 83.974 \\
$\mathrm{a}$ & $\mathrm{mm}$ & 18 & 18.0267 & 18.177 \\
$\mathrm{~b}$ & $\mathrm{~mm}$ & 38.0 & 38.056 & 38.206 \\
$\mathrm{~L}$ & $\mathrm{~mm}$ & 103.75 & 103.904 & 103.904 \\
$\mathrm{~L}_{\text {end }}$ & $\mathrm{mm}$ & 106.971 & 107.13 & 107.13 \\
$\mathrm{~B}_{\text {end }}$ & $\mathrm{mm}$ & 84.5 & 84.625 & 84.4752 \\
$\mathrm{a}_{\text {end }}$ & $\mathrm{mm}$ & 20.0 & 20.03 & 20.18 \\
$\mathrm{~b}_{\text {end }}$ & $\mathrm{mm}$ & 39.5 & 39.558 & 39.708 \\
\hline
\end{tabular}

RF parameters for operating modes are also calculated for design beta and optimal beta and summarized in Table 3.14. Maximum possible gradient for peak magnetic field of $70 \mathrm{mT}$ is $19 \mathrm{MV} / \mathrm{m}$. Surface electric field and surface magnetic field distribution for fundamental modes are shown in Figures 3.35a and 3.35b respectively. Fields are normalized to $1 \mathrm{~mJ}$ stored energy in cavity. It can be noticed that magnitude of peak 
surface fields are less in end cell than in inner cell. It is done in order to reduce field emission possibility.

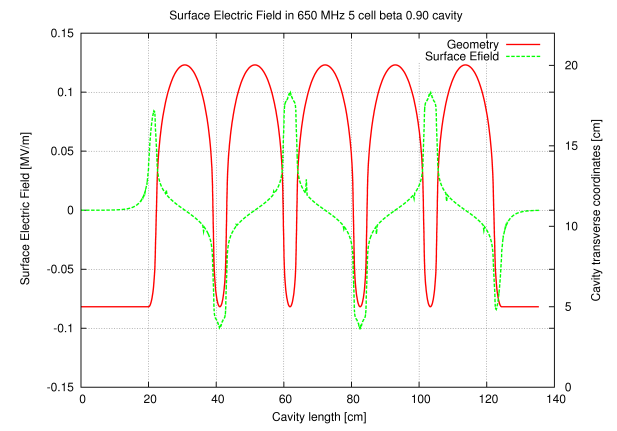

(a)

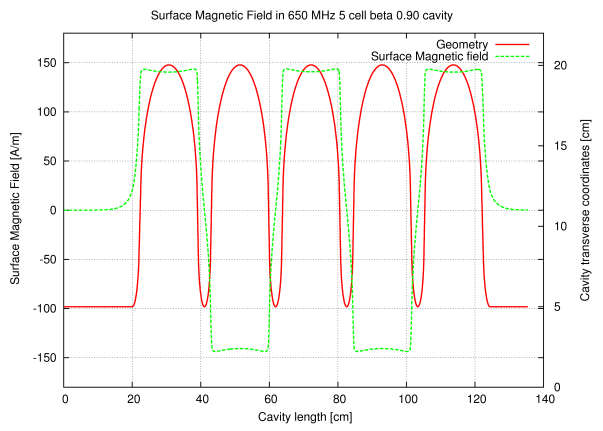

(b)

Figure 3.35: Surface fields distribution in $\beta_{G}=0.90$ cavity-(a) Surface electric field and (b) surface magnetic field.

Table 3.14: RF Parameters for fundamental mode of 5-cell, $\beta_{G}=0.90,650 \mathrm{MHz}$ cavity.

\begin{tabular}{cccc}
\hline Parameters & Unit & \multicolumn{2}{c}{ Magnitude } \\
\hline Coupling coefficient & $\%$ & 0.75 & 0.75 \\
$E_{p} / E_{a c c}$ & - & 2.08 & 2.03 \\
$B_{p} / E_{a c c}$ & $\mathrm{mT} /(\mathrm{MV} / \mathrm{m})$ & 3.79 & 3.68 \\
$\mathrm{R} / \mathrm{Q}$ & $\Omega$ & 607 & 642 \\
$\mathrm{G}$ & $\Omega$ & 255 & 255 \\
Frequency & $\mathrm{GHz}$ & 650 & 650 \\
Field flatness & $\%$ & 0.6 & 0.6 \\
Active length & $\mathrm{mm}$ & 1042.94 & 1042.94 \\
\hline
\end{tabular}

HOMs spectrum is also studied to investigate any potentially concerned mode with high effective impedance. Figures 3.36a and 3.36b show monopole and dipole mode spectrum of $650 \mathrm{MHz}, 5$-cell, $\beta_{G}=0.90$ cavity for designed beta. It can be seen that operating mode has highest effective impedance of $607 \Omega$. Next highest impedance for monopole modes is $\sim 130 \Omega$ and transverse impedance of dipole modes is reached to $60 \mathrm{k} \Omega / \mathrm{cm}^{2}$. Variation in HOMs impedances of most concerned monopole and dipole 


\section{DESIGN OF SCRF CAVITY FOR THE PROJECT-X LINAC}

modes with operating range of cavity is shown in Figures $3.37 \mathrm{a}$ and $3.37 \mathrm{~b}$ respectively.

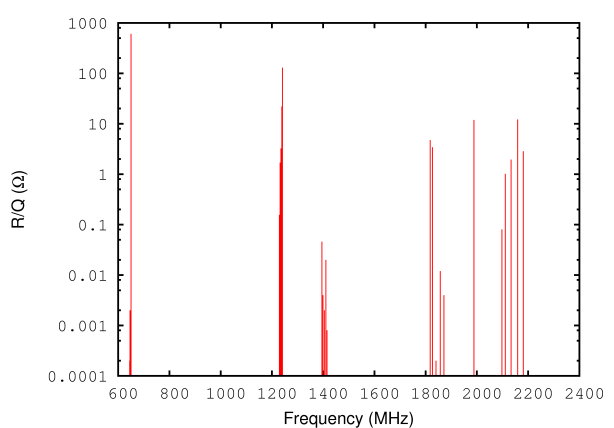

(a)

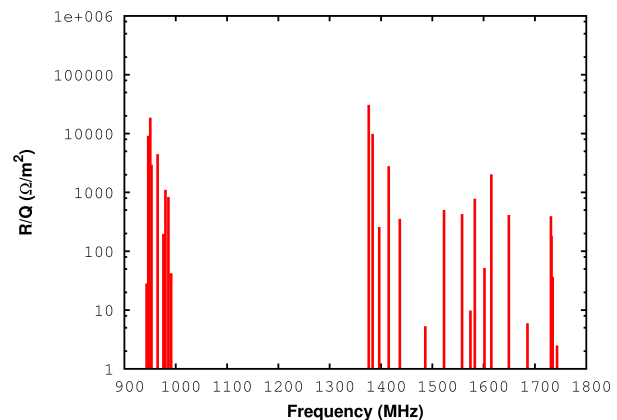

(b)

Figure 3.36: (a)Distribution of effective impedance R/Q $(\Omega)$ of longitudinal modes (monopole modes) and (b) transverse effective impedance $(R / Q)_{\|}\left(\Omega / \mathrm{cm}^{2}\right)$ of transverse modes (dipoles) for $\beta_{G}=0.90$.

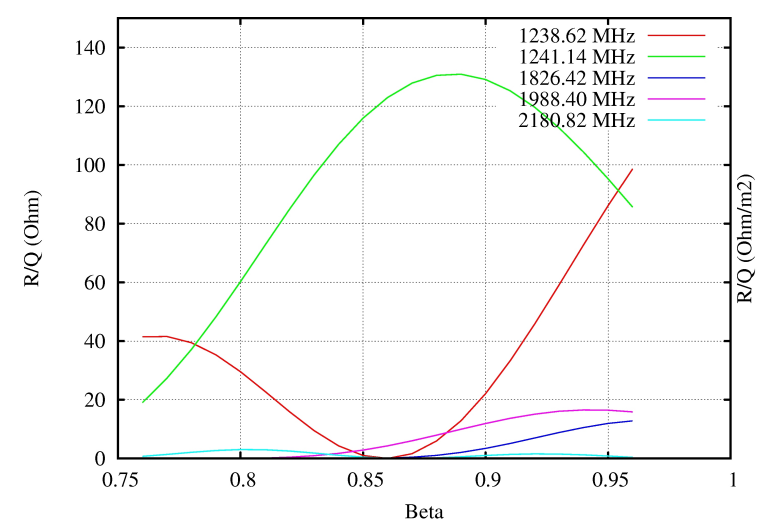

(a)

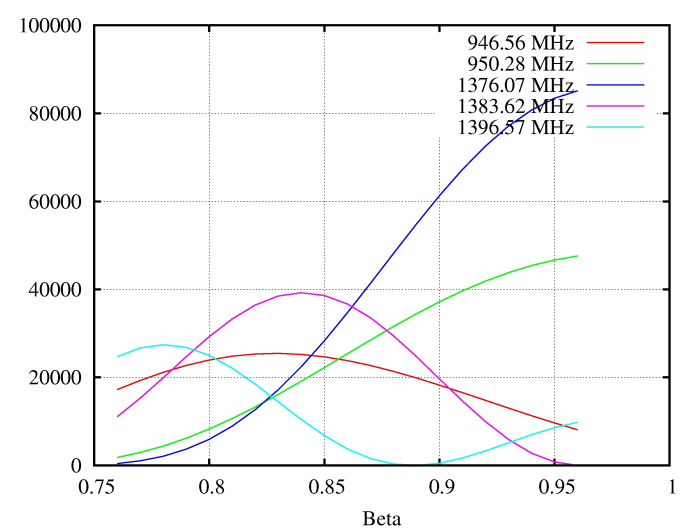

(b)

Figure 3.37: Variation in HOM impedances with $\beta$ - for most concerned (a) monopole modes and (b) dipole modes for $650 \mathrm{MHz}, \beta_{G}=0.90,5$-cell cavity.

Monopole and dipole dispersion curves for first six pass bands are shown in Figures 3.38 and 3.39 respectively. It can be seen that frequency bandwidth of monopole fifth pass band is about $50 \mathrm{KHz}$ and dispersion curve looks very flat. As all cells are not identical due to fabrication errors, therefore, there is frequency spread between cells 


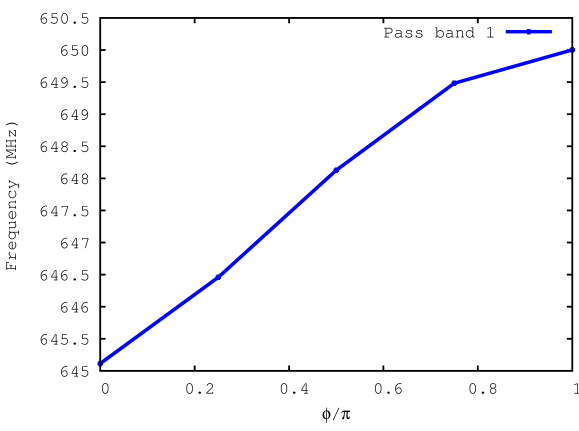

(a)

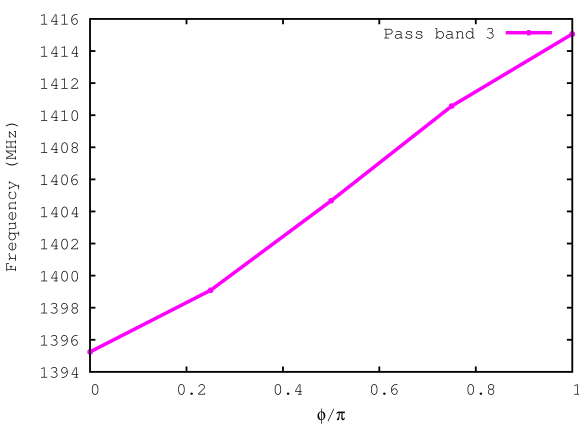

(c)

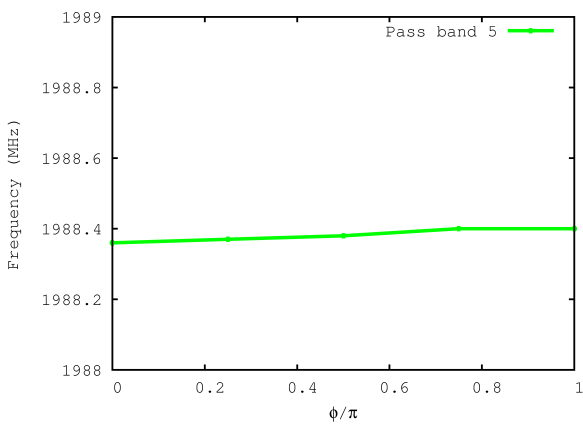

(e)

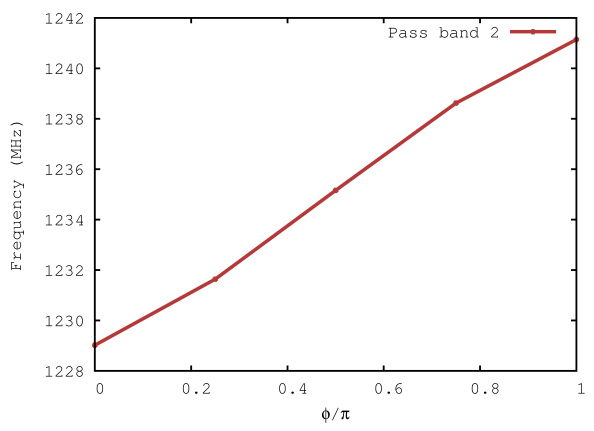

(b)

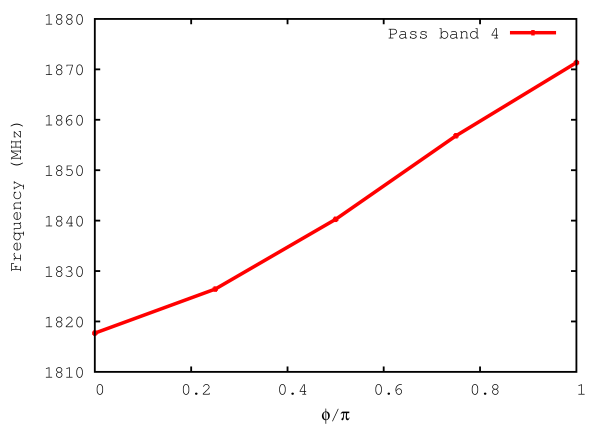

(d)

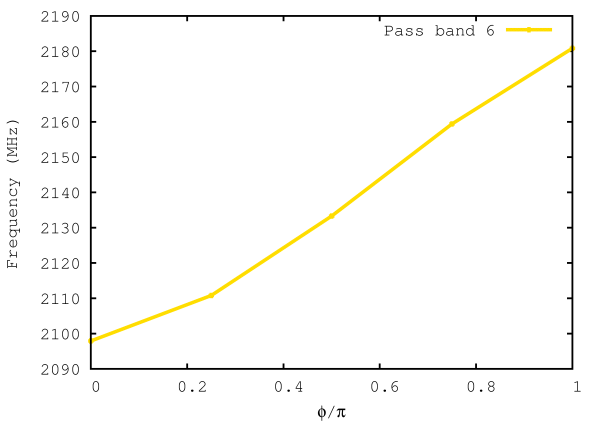

(f)

Figure 3.38: Distribution of monopole modes (longitudinal modes) with phase advance for first six pass band of $\beta_{G}=0.90,650 \mathrm{MHz}, 5$-cell cavity. 


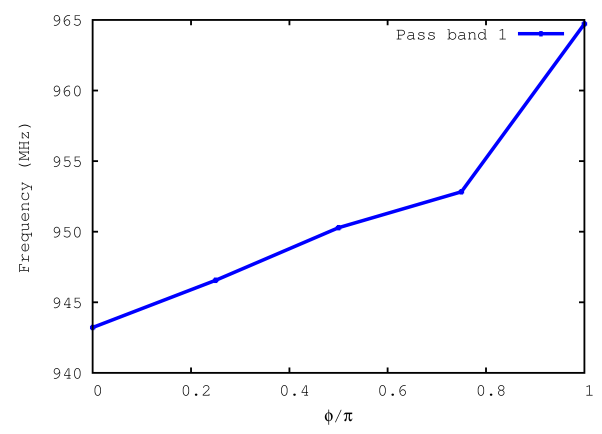

(a)

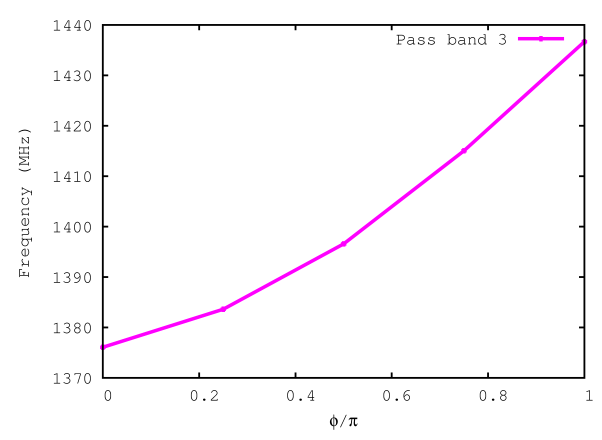

(c)

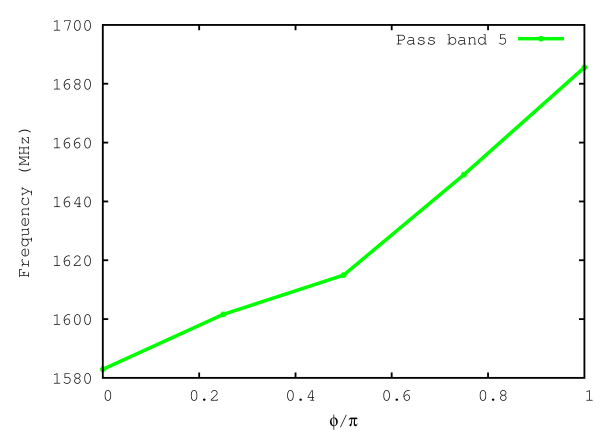

(e)

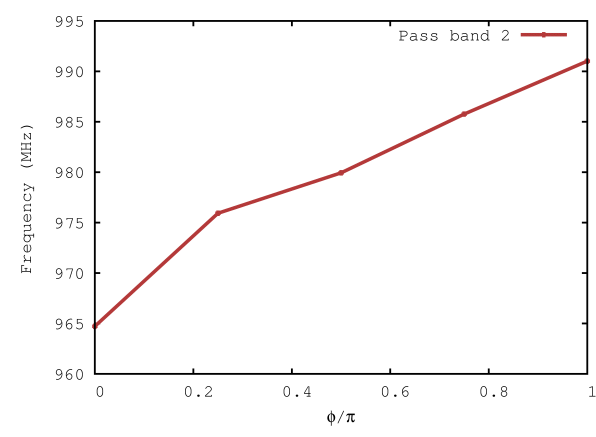

(b)

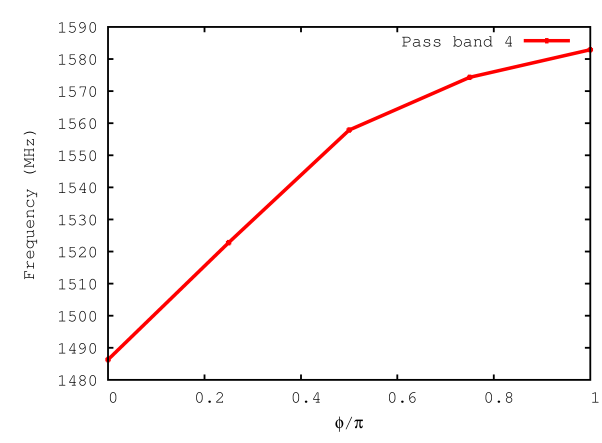

(d)

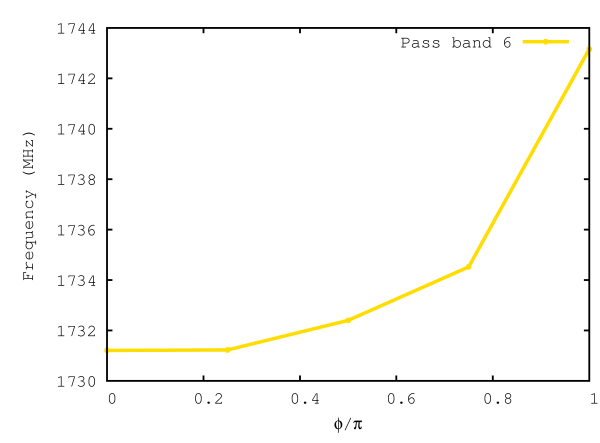

(f)

Figure 3.39: Distribution of dipole modes (transverse modes) with phase advance for first six pass band of $\beta_{G}=0.90,650 \mathrm{MHz}, 5$-cell cavity. 
and it is typically of the order of $1 \mathrm{MHz}$. In this case there will be mixing of modes for fifth monopole pass band which will result in trapping of modes. However, these losses might be manageable for $1 \mathrm{~mA}$ average beam current but possibility of future upgrade of $\mathrm{CW}$ linac to deliver $5 \mathrm{~mA}$ average beam current requires increase in bandwidth to avoid huge cryogenic losses. Alternative of $\beta_{G}=0.90$ cavity are designed with large $R_{\text {iris }}$. It is to be noted that increase in $R_{\text {iris }}$ results in increase in cell to cell coupling and therefore, bandwidth of passband. Figure 3.40a shows variation of bandwidth of fifth monopole pass band with $R_{\text {iris }}$. It can be seen that bandwidth larger than $1 \mathrm{MHz}$ can be obtained for $R_{\text {iris }}$ larger than $62 \mathrm{~mm}$. $R_{\text {iris }}$ also affects acceleration efficiency of cavity. Thus, large increase in $R_{\text {iris }}$ is not effective from cavity RF performance and fabrication cost point of view.

To understand the nature of variation in bandwidth of fifth pass band with length of cavity, $\beta_{G}$ is varied. Figure $3.40 \mathrm{~b}$ shows variation in bandwidth with different $\beta_{G}$ of cavity. It can be seen than bandwidth is minimum for $\beta_{G}=0.90$. Small changes in either side of this beta results in significant increase in bandwidth. Thus, alternative design of cavity with different $\beta_{G}$ is better solution to deal with narrow bandwidth of fifth pass band of $\beta=0.90$ cavity.

\subsubsection{Requirement of HOM damper for $650 \mathrm{MHz}$ cavities}

Each time beam is passed through cavity, it induces HOMs. Resonance excitation of HOM results in degradation of beam quality (beam emittance) and increased power losses. Therefore, HOM damper (HOM coupler) is used to extract these modes out of cavity (avoiding resonance excitation) and thus, reduces power dissipation in SCRF cavity. However, HOMs damper is an expensive and complicated part. Assembly of HOM damper requires additional hardware, cables and wires which adds further complication in fabrication of SCRF cavity. It can also add problems such as multipacting, leaks, loads etc. in cavity. Analysis of HOMs effect provides better understanding about the requirements of $\mathrm{HOM}$ damper for 5-cell, $650 \mathrm{MHz}$, elliptical cavities. Absence of HOM coupler will reduced the cost of accelerating structure to large extent. Study is performed to analyzed HOMs effect in intermediate $\left(\beta_{G}=0.61\right)$ and high energy $\left(\beta_{G}=0.90\right)$ sections of CW linac for the Project-X facility. 


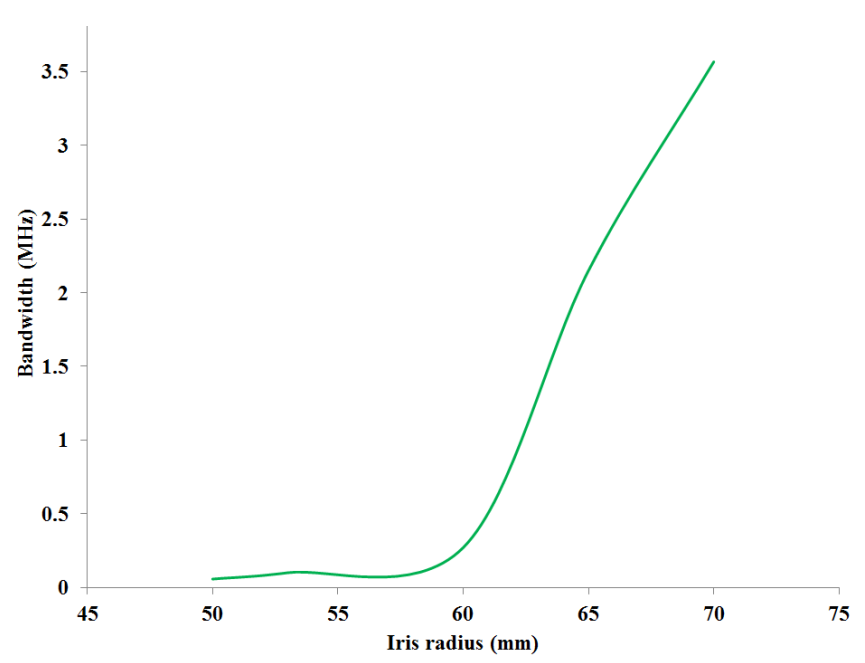

(a)

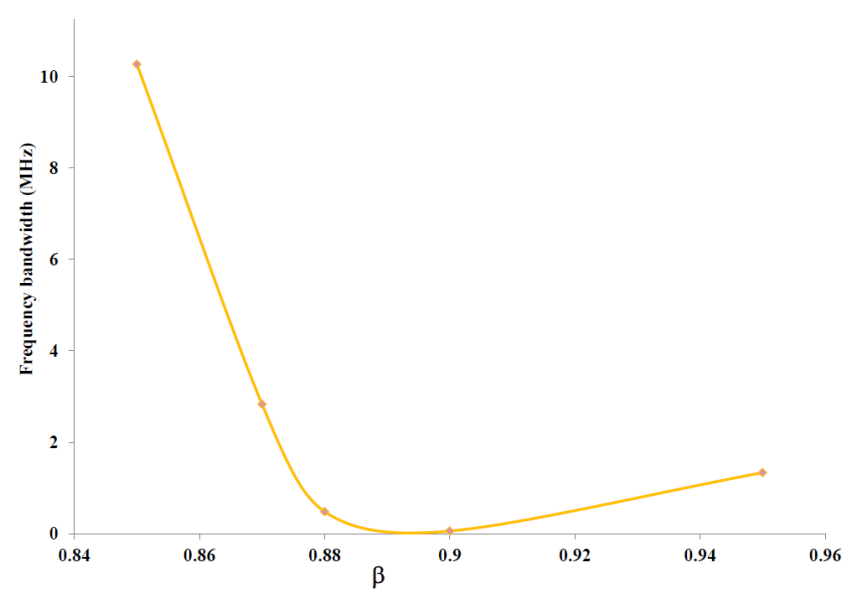

(b)

Figure 3.40: Variation in frequency bandwidth of fifth pass band with (a) $R_{\text {iris }}$ and (b) with $\beta_{G}$.

\subsubsection{Beam Time Structure}

The CW linac of the Project $\mathrm{X}$ provides $\mathrm{H}^{-}$beam with average current of $1 \mathrm{~mA}$ and has a special time structure in order to satisfy the requirements of the experiments simultaneously. One whole period of beam structure is shown in Figure 1.9 and separation scheme of the $\mathrm{H}^{-}$beam from the linac to three different experiments: Mu2e, Kaon, and other is shown in the Figure 3.41 .

Each bunch contains $9 \times 10^{7} \mathrm{H}^{-}$ions. The bunch sequence frequency for the Mu2e 


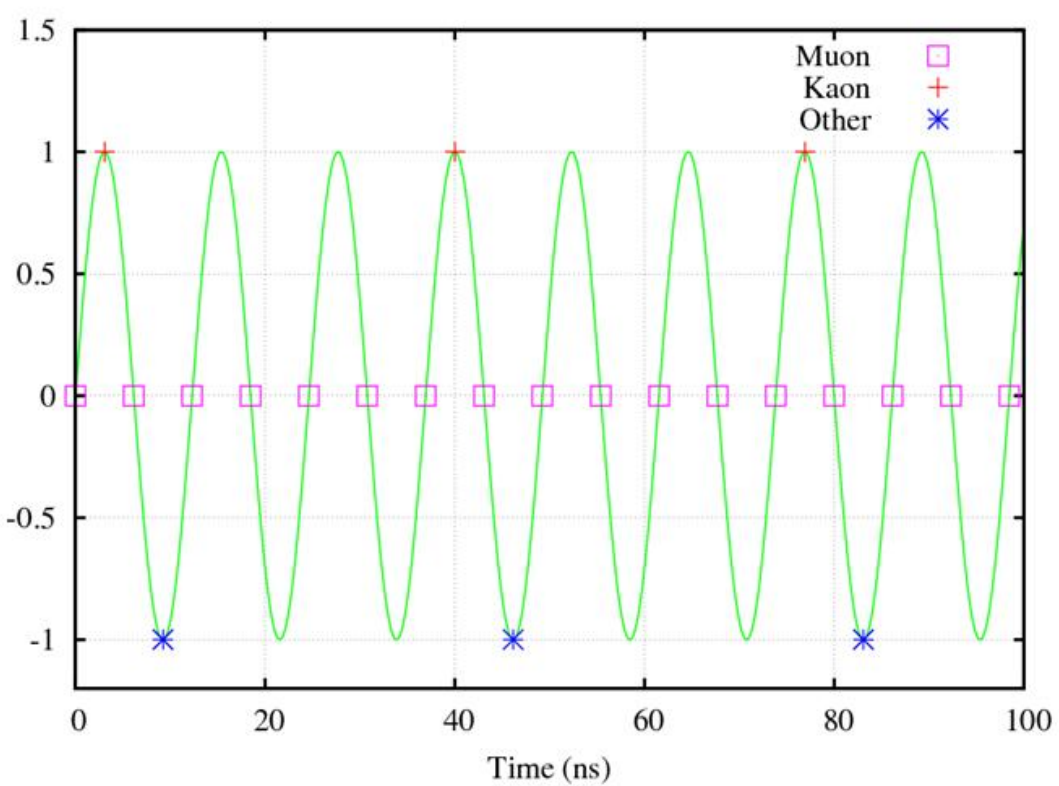

Figure 3.41: Beam separation scheme at the end of CW linac at Project-X facility.

experiment is $162.5 \mathrm{MHz}$ (for the RFQ frequency of $325 \mathrm{MHz}$ ) and the bunch train width is $100 \mathrm{nsec}$ when the train repetition rate is $1 \mathrm{MHz}$. The bunch sequence for Kaons and other experiment is $27.08 \mathrm{MHz}$. The beam power for Mu2e is $400 \mathrm{~kW}$, and $800 \mathrm{~kW}$ for two other experiments. Thus, the beam current spectrum contains:

- Harmonics of the bunch sequence frequency of $27.08 \mathrm{MHz}$.

- Sidebands of the harmonics of $162.5 \mathrm{MHz}$ separated by $1 \mathrm{MHz}$ as shown in Figure 3.42 ,

The idealized spectrum of the beam is shown in the Figure 3.42 . The beam imperfectness like finite time of bunches and jitter effects are not taken into account. One can see that it has spectral components with large amplitude every $325 \mathrm{MHz}$, components with smaller amplitudes about every $27 \mathrm{MHz}$, and sidebands about every $1 \mathrm{MHz}$.

\subsubsection{Resonance excitation of HOMs}

As mentioned earlier, when beam passes through the cavity, it extracts not only the RF energy but also excites HOMs. If frequencies of these modes come close to eigen mode frequencies of cavity, it results in excitation of resonance of HOMs. It may lead 


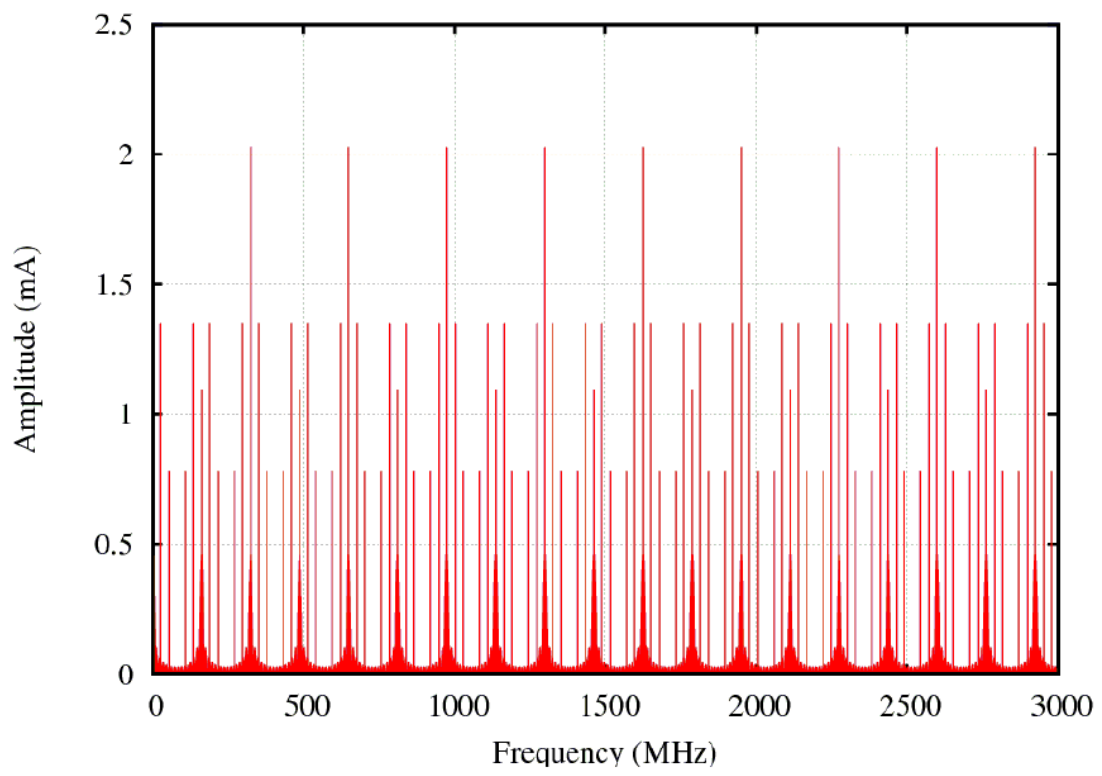

Figure 3.42: Beam spectrum for Project-X CW linac.

to induce large voltage which can act back on beam and dilutes beam quality. HOMs are characterized by $R / Q$ and $Q_{e x}$. Higher the $Q_{e x}$, higher will be power deposited in the cavity.

Power losses due to HOMs: The main accelerating mode is compensated by feeding power from external RF source but in the absence of HOM damper, HOMs decay inside cavity. Excess of power dissipation can result in quench of cavity or increase cryogenic losses. To calculate these power losses, consider that an average alternating current, $\tilde{I}_{n}$, with angular frequency, $\omega_{n}$, passing through the cavity excites HOM with angular frequency $\omega_{m}$, and impedance, $(R / Q)_{m}$, with resulting magnetic field $\vec{H}_{n m}$ which is given as [65]:

$$
\vec{H}_{n m}=\frac{\omega_{m} \sqrt{\omega_{m}}}{i\left(\omega_{n}^{2}-\omega_{m}^{2}-i \frac{\omega_{m} \omega_{n}}{Q_{m}}\right)} \cdot \frac{\tilde{I}_{n}}{2 \sqrt{U_{m}}} \cdot \sqrt{\left(\frac{R}{Q}\right)_{m}} \vec{H}_{m}
$$

where $\vec{H}_{m}$ is the the eigenfunction of the mode normalized to energy stored in the cavity $\left(U_{m}\right)$ and $Q_{m}$ is quality factor. The accelerator definition of $R / Q_{m}$ is used i.e., $(R / Q)_{m}=V_{m}^{2} / \omega_{m} U_{m}$, where $\mathrm{V}_{m}$ is voltage induced by mode inside cavity. If the HOM spectrum is known, one can calculate the total magnetic field excited by a 
current harmonic $\tilde{I}_{n}$. It is given as:

$$
\vec{H}^{(n)}=\sum_{m} \vec{H}_{n m}
$$

Thus, total power losses (P) caused by HOMs excitation can be calculated from the following:

$$
P=\sum_{n} \frac{R_{n}}{2} \oint \vec{H}^{(n)} \vec{H}^{*(n)} d s,
$$

where $R_{n}$ is surface resistance, which is expressed as:

$$
R_{n}=R_{B C S}^{n}+R_{\text {residual }}
$$

Residual resistance is considered $10 \mathrm{n} \Omega$ for calculation and average BCS resistance is

$$
R_{B C S}^{n}=\frac{2.10^{-4}}{T[K]}\left(\frac{f_{n}[G H z]}{1.5}\right)^{2} \exp \left(-\frac{17.67}{T[K]}\right)
$$

where $f_{n}$ is frequency of beam spectrum line and $T=2 \mathrm{~K}$.

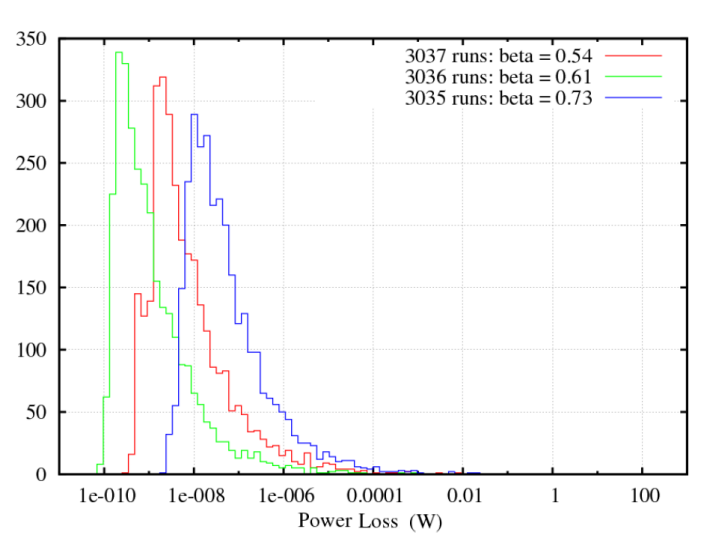

(a)

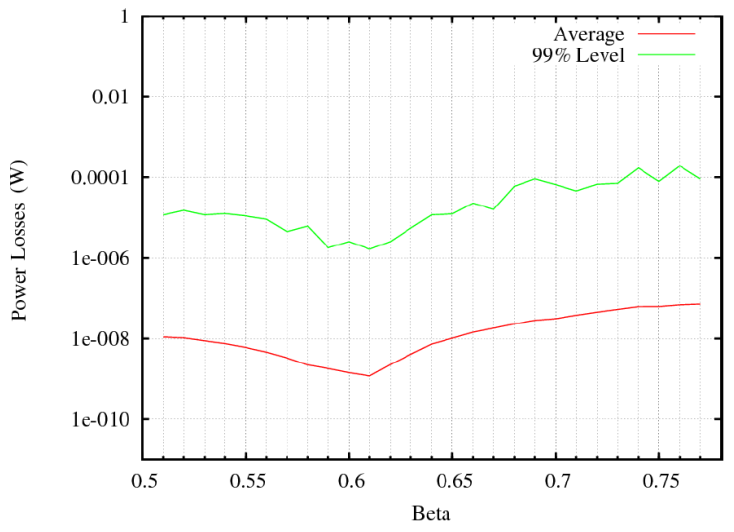

(b)

Figure 3.43: (a)Power loss distribution for the $\beta_{G}=0.61$ cavity for $\mathrm{Q}=10^{10}$. $\beta=$ $0.61, \beta=0.54$ and $\beta=0.73$ are considered for calculation of power losses.(b) Power losses due to HOM excitation for $\beta=0.61$ cavity as a function of beam velocity $(\beta)$.

The dependence of power loss on the beam energy was studied. Quality factors for the HOMs ranging from $10^{7}$ to $10^{10}$ were considered. Based on the predicted deviations of HOMs frequency and $R / Q$ we generated 3000 random linacs in order to estimate the probability of power losses. Simulation is performed for both $\beta_{G}=0.61$ and $\beta_{G}=0.90$ cavities. Calculation is performed for particular values of beta which cover complete 


\section{DESIGN OF SCRF CAVITY FOR THE PROJECT-X LINAC}

range of energy for which these cavities will be used. The power loss distribution for the case of the $\beta_{G}=0.61$ cavity for the conservative case of maximum value of $Q=$ $10^{10}$ (for all modes) is shown in Figure 3.43a. Power losses are minimum when all HOM frequencies are far from beam spectrum lines. When one (and sometimes more) of HOMs frequencies are closer to main beam spectrum lines, resonances occur and field amplitude of these HOMs may become very high which results in high power losses. However, it can be seen from figure 3.43a that most of simulated cases have lower power losses which imply lower possibility of excitation of resonances. The statistical straggling of RF losses is higher than the dependence on beam energy. Thus, we can neglect the effect of RF losses variation along the linac.

Power losses as a function of beam velocities $(\beta)$ is shown in Figure $3.43 \mathrm{~b}$, It can be noticed that $99 \%$ of simulated cases show losses below green line in Figure $3.43 \mathrm{~b}$. Power losses due to HOM excitation are also studied for $\beta_{G}=0.90$ cavity and is shown in Figure 3.44 .

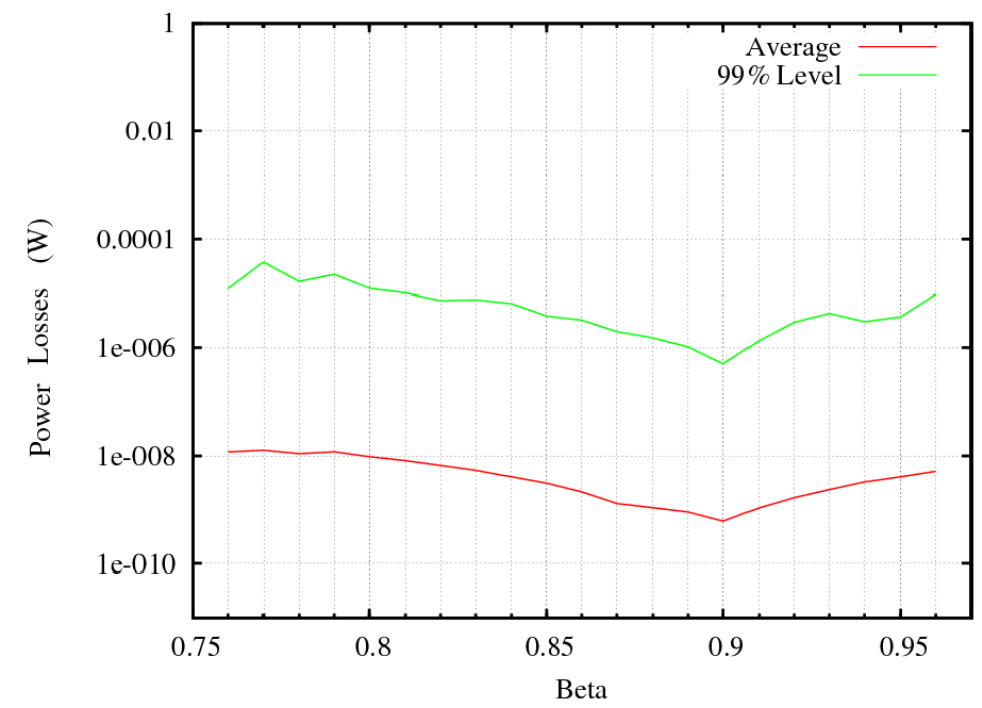

Figure 3.44: Power losses in $\beta_{G}=0.90,650 \mathrm{MHz}, 5$-cell cavity due to HOM excitation as a function of beam velocity $(\beta)$.

If a HOM frequency is in resonance with a strong beam spectrum line, losses are significant. In this case resonance can be avoided by detuning cavity by few ten of $\mathrm{KHz}$ and then tuning it back for operating mode. This process results in change in HOM frequency by few hundred of $\mathrm{KHz}$ and hence resonance can be avoided. The detail is 
presented in reference [66]. Figure 3.45 also confirms that even a $50 \mathrm{~Hz}$ displacement

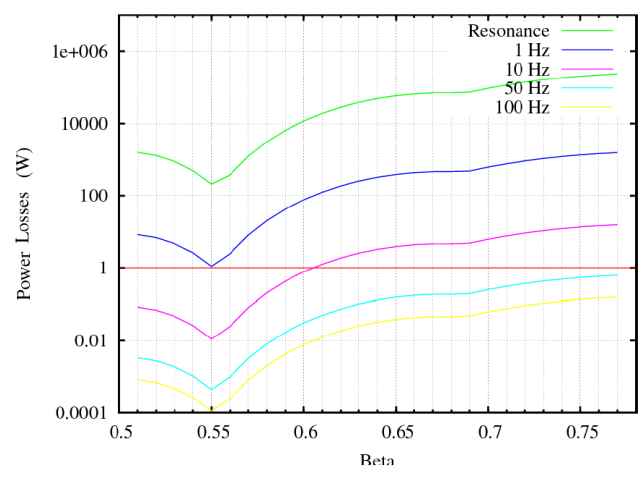

(a)

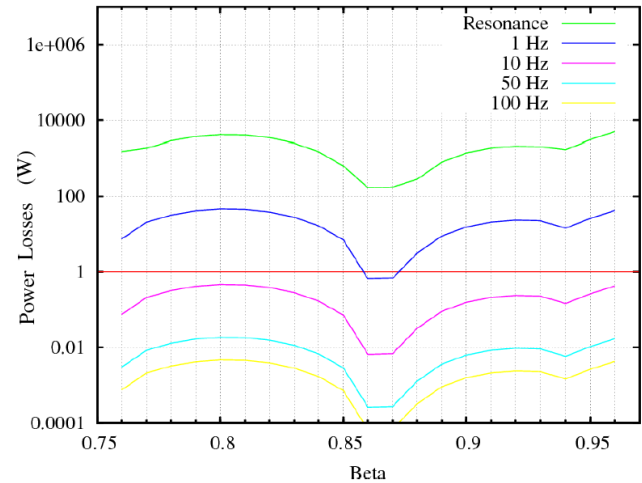

(b)

Figure 3.45: Power loss reduction by HOM frequency shift for a (a) $\beta_{G}=0.61$ and (b) $\beta_{G}=0.90$ cavity as a function of $\beta$ for $\mathrm{Q}=10^{10}$.

from the resonance is enough to reduce losses down to an acceptable level of $1 \mathrm{~W}$ per cavity for all $\beta$.

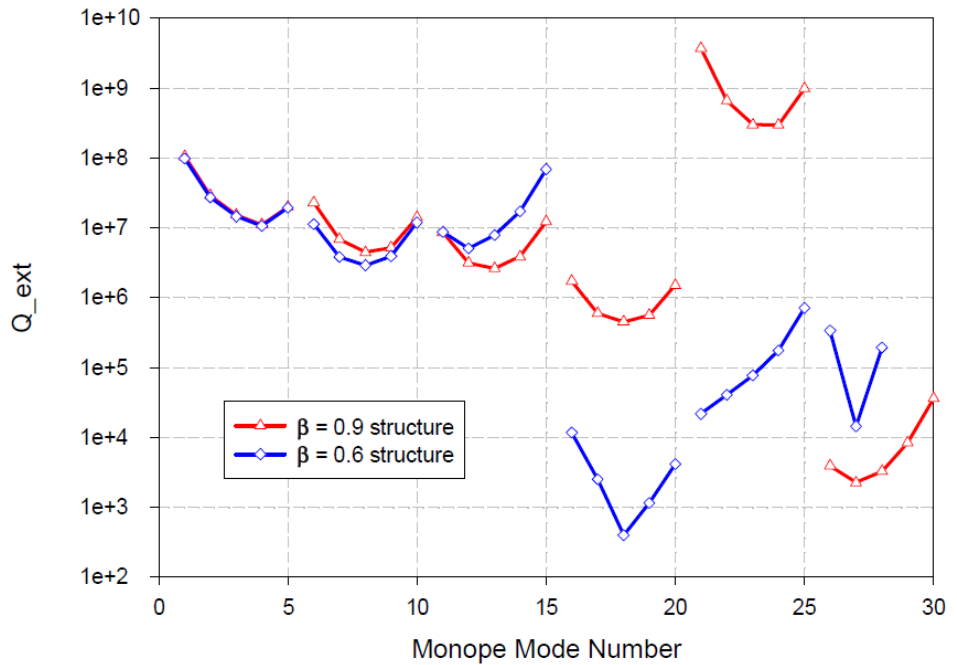

Figure 3.46: External quality factor for monopole pass band of $\beta_{G}=0.90$ and $\beta_{G}=0.61$ cavity.

A similar approach is used to estimate power losses due to propagating HOMs whose frequencies are higher than beam pipe cut-off frequencies. HOMs up to frequency 5.7 $\mathrm{GHz}$ are accounted for power losses estimation. Evaluation of power loss is performed 


\section{DESIGN OF SCRF CAVITY FOR THE PROJECT-X LINAC}

with the conservative consideration that all the modes are trapped and contribute to power losses. Length of beam pipe is varied in order to find maximum value of $R / Q$. Losses per cavity is found of the order of few $\mu \mathrm{W}$ in walls and approximately $50 \mathrm{~mW}$ in bellows for $Q$ in $10^{6}-10^{9}$ range. As estimation was performed for extreme case, one can conclude that power losses due to propagating modes are negligible.

$\mathrm{Q}_{e x}$ of HOMs are calculated using a complex eigenmode solver with matched boundary conditions on coupler outputs. $Q_{e x}$ for monopole pass band of $\beta_{G}=0.61$ and $\beta_{G}$ $=0.90$ cavity are shown in Figure 3.46. The fact, that monopole HOMs are loaded to the main power coupler, limits their quality factors to $10^{7}-10^{8}$. The only exception is the fifth pass band of the high energy cavity, which contains a mode with Quality factor greater than $10^{9}$

Estimation of transverse emittance growth Estimation of emittance growth due to excitation of dipole modes are performed. One cavity model is considered in which it is assumed that only one cavity has kick voltage. This model can be used for estimation of the maximum value of the emittance dilution in the situation when collective effects do not occur.

If the cavity has transverse misalignment $x_{0}$, transverse kick voltage $\left(V_{k i c k}\right)$ induced by excited $\mathrm{HOM}$ of frequency $\omega=2 \pi f$ with transverse impedance $(R / Q)_{\perp}$ and quality factor $Q$ is given as following [65]:

$$
V_{k i c k}=\frac{c}{4 \pi}\left(\frac{R}{Q}\right)_{\perp} \cdot \frac{x_{0} \tilde{I} f}{f^{2}-f_{0}^{2}-i \frac{f f_{0}}{Q}} ;
$$

where $c$ ia velocity of light, $\tilde{I}$ is component of alternating current with frequency $\omega=$ $2 \pi f_{0}$. If $\Delta f=f-f_{0}$ and $\Delta f / f_{0} \gg 1 / Q$, kick voltage can be approximated as:

$$
V_{k i c k} \approx \frac{c}{4 \pi}\left(\frac{R}{Q}\right)_{\perp} \cdot \frac{x_{0} \tilde{I}}{2 \Delta f} .
$$

Transverse emittance is given as $\epsilon_{t}=\beta \gamma \sigma_{x} \sigma_{\dot{x}}$ where:

$$
\begin{aligned}
\sigma_{x} & =\beta_{f} \sigma_{\dot{x}} \\
\sigma_{\dot{x}} & =\frac{e V_{k i c k}}{\sqrt{2} p c} ;
\end{aligned}
$$

$p=\gamma \beta m c, \beta$ and $\gamma$ are Lorentz parameters, $\beta_{t}$ is betatron function, $\sigma_{x}$ and $\sigma_{\dot{x}}$ are beam transverse size and transverse divergence respectively. As bunch length $\sigma_{z} \ll \lambda_{H O M}$, 
HOM kick is same for all particles in the bunch but different for different bunches. Thus, this HOM does not increase emittance of an individual bunch, but gives kicks in different phase for different bunches increasing total phase space area occupied by the bunches. Transverse emittance dilution due to transverse kick can be now written as:

$$
\Delta \epsilon_{t}=\frac{\beta_{t}}{\gamma \beta}\left(\frac{e x_{0} \tilde{I}}{8 \sqrt{2} \pi m c \Delta f}\left(\frac{R}{Q}\right)_{\perp}\right)^{2} .
$$

To limit the emittance growth $\Delta \epsilon_{t} \ll \epsilon_{\text {initial }}$, HOM frequency deviation from beam spectrum line should be

$$
\Delta f \gg \frac{e x_{0} \tilde{I}}{8 \sqrt{2} \pi m c}\left(\frac{R}{Q}\right)_{\perp} \sqrt{\frac{\beta_{f}}{\beta \gamma \epsilon_{\text {initial }}}}=\delta f_{\epsilon} .
$$

Assuming normal distribution of HOM frequency, one can find probability $(\wp)$ to have emittance dilution more or equal to initial emittance as:

$$
\wp=\frac{e x_{0} \tilde{I}}{16 \pi \sqrt{\pi} m c \sigma}\left(\frac{R}{Q}\right)_{\perp} \sqrt{\frac{\beta_{f}}{\beta \gamma \epsilon_{\text {initial }}}} \cdot e^{-\frac{\Delta f^{2}}{2 \sigma^{2}}} ;
$$

where $\Delta f$ is distance between HOM frequency and a beam spectrum line. The value of beta function at $650 \mathrm{MHz}$ sections can be considered as $15 \mathrm{~m}$. Maximum misalignment can be estimated as $1 \mathrm{~mm}$ and $m$ is mass of $\mathrm{H}^{-}$particle. For the worst case, $f_{0}=$ $1376 \mathrm{MHz},\left(\frac{R}{Q}\right)_{\perp}=60 \mathrm{k} \Omega / \mathrm{m}^{2}$, beam energy $=500 \mathrm{MeV}$ and $\tilde{I}=0.5 \mathrm{~mA}$, Frequency spread between HOM and beam spectral line must be: $\Delta f \gg 2.5 \mathrm{~Hz}$ to maintain emittance growth very small relative to initial emittance.

The dependence of $\delta f_{\epsilon}$ on beam velocity $(\beta)$ is shown for $\beta_{G}=0.61$ and $\beta_{G}=0.90$ in Figures $3.47 \mathrm{a}$ and $3.47 \mathrm{~b}$ respectively. Probability that HOM contributes significantly to the emittance growth in intermediate and high energy section of linac is also calculated and its variation with beam energy is shown in Figures 3.48a and 3.48b for $\beta_{G}=0.61$ and $\beta_{G}=0.90$ cavities respectively. The maximum probability that HOM results in significant emittance growth is of the order of $10^{-6}$ which is extremely small. It should also be accounted that frequency detuning and tuning of operating mode can also be employed to avoid the resonance in cavities. It further reduces risks of significant emittance dilution in CW linac due to HOM.

\section{Estimation of longitudinal emittance growth}

A similar approach is adopted for emittance dilution due to resonant excitation of longitudinal HOM. If $\epsilon_{z}$ is initial longitudinal emittance $\left(1.5 .10^{3}\right.$ nsec.eV $), \sigma_{t}$ is bunch 


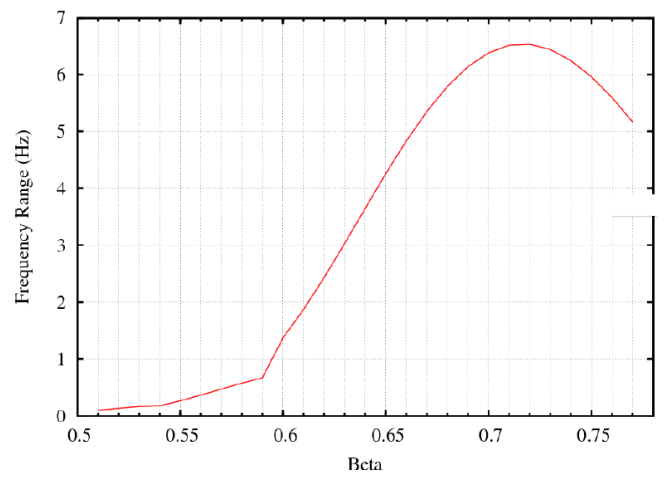

(a)

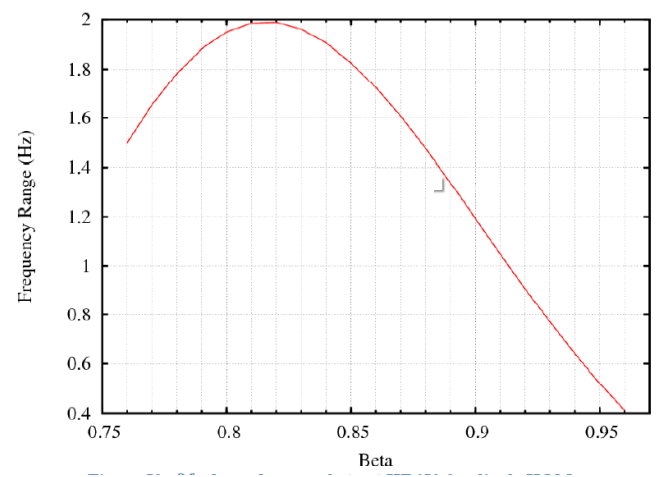

(b)

Figure 3.47: $\delta f_{\epsilon}$ dependence on $\beta$ velocity for (a) $\beta_{G}=0.61$ and (b) $\beta_{G}=0.90$ cavity.

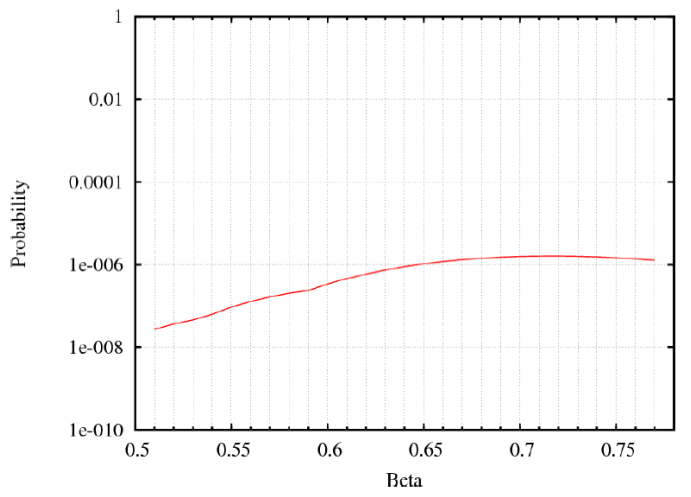

(a)

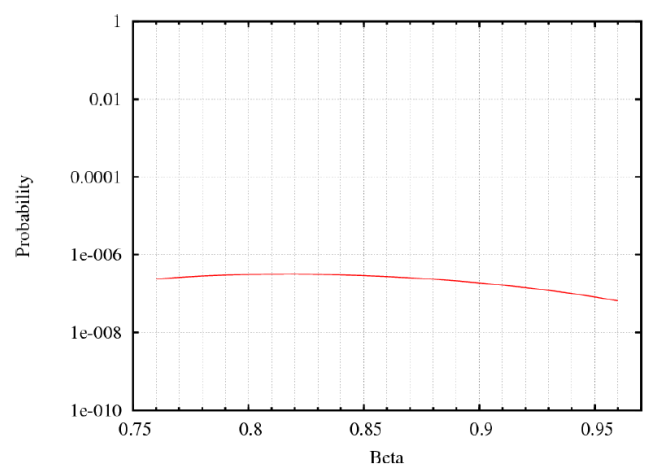

(b)

Figure 3.48: Probability of having significant emittance growth for (a) $\beta_{G}=0.61$ and (b) $\beta_{G}=0.90$ cavity.

length (0.011 nsec) and $U_{H O M}$ is average energy gain caused by HOM, emittance dilution because of longitudinal HOM is $\delta \epsilon_{z}=\sigma_{t} \cdot U_{H O M}$. For high $Q$ resonances $U_{H O M}$ is given as:

$$
U_{H O M}=\frac{\tilde{I}}{4 \sqrt{2} \delta f / f} .\left(\frac{R}{Q}\right) ;
$$

and thus, to ensure that HOMs do not cause significant emittance growth, i.e., $\delta \epsilon_{z} \ll \epsilon_{z}$, we get the following condition:

$$
\delta f \gg f \sigma_{t} \frac{\tilde{I}}{4 \sqrt{2} \epsilon_{z}} \cdot\left(\frac{R}{Q}\right) .
$$

For the worst case, i.e., beginning of the high beta $650 \mathrm{MHz}$ section, $\sigma_{t}=7.7 \times 10^{-3}$ 
nsec, $\tilde{I}=0.5 \mathrm{~mA}, R / Q=130 \Omega$ for HOM with frequency of $1241 \mathrm{MHz}$, we get $\delta f \gg 70$ $\mathrm{Hz}$. The gain caused by HOM is $<300 \mathrm{keV}$, which is much smaller than the operating mode gain of $20 \mathrm{MeV}$. This results in a very small power loss of $0.15 \mathrm{~W}\left(Q_{0}=5.10^{9}\right)$ and hence does not contribute to the cryogenic losses. If the HOM is in resonance then the Q-factor has to be less than $1.8 \times 10^{7}$. HOM frequency has to differ $\sim 70 \mathrm{~Hz}$ of the spectrum line in order to provide significant emittance dilution. Even in the case when it happens, it is possible to move the HOM frequency away from the spectrum line by simply detuning the cavity by tens of $\mathrm{kHz}$, and then tune the operating mode back to the resonance.

\subsubsection{Collective effects in CW linac}

It is considered that collective effect should not be an issue in CW ion linac because of the following:

- Ion linac generally use families of cavity to accelerate the beam for given energy range. Each families have different frequencies and HOM spectrum.

- Fundamental mode of cavities are tuned to operating frequencies via various means (mechanical tuning, tuner etc.) but HOM spectrum in each cavity are different due to manufacturing errors. Frequency spread of HOMs in each cavity is in the range of 5-8 MHz.

- Beam current is relatively low thus collective effects are less probable.

- Ion beam velocities change over the linac and hence effective impedance of cavity (R/Q) also varies.

- Linac length is shorter. Thus, probability of building of collective effects such as beam break up (BBU) are much smaller.

Studies have been performed to analyze HOMs impact on beam quality and power dissipation for extreme cases. Results are summarized as follows:

- Studies show that cryogenic power losses due to HOMs are not significant. Average power losses for both $\beta_{G}=0.61$ and $\beta_{G}=0.90$ cavity are well below 1 $\mathrm{mW}$. 


\section{DESIGN OF SCRF CAVITY FOR THE PROJECT-X LINAC}

- Emittance dilution, both in longitudinal plane and transverse plane are very small and the probability of is significant emittance growth is very low even under the conservative approach.

- Beam current is small. Thus, impact of collective effects such as BBU are less probable.

Lower influence of HOM on beam quality and power dissipation opens the possibilities of operation of $650 \mathrm{MHz}$ cavities without HOM coupler. Further, any accidental excitation of resonance can be avoided by tuning and detuning of operational mode. This will result in shifting of HOMs frequency spectrum away from resonance region. Avoiding HOM coupler simplifies cavity operation and reduces the potential threat of multipacting and other problems which may limit cavity performance. Another safe option is to make flanges ( $\sim 40 \mathrm{~mm}$ ) for HOM dampers, seal them and install the dampers in future for possible upgrade if necessary. 


\section{4 \\ Beam dynamics study of Project-X CW linac}

This chapter introduces the layout of Project-X CW linac. Principles and assumptions used for lattice design of the CW linac are discussed. Beam dynamics studies are performed for baseline design using beam dynamics codes like TRACEWIN and TRACK. Beam quality parameters such as emittance dilution, beam trajectory etc. are analyzed. Operation of ion linac in $\mathrm{CW}$ regime puts stringent tolerances on beam line elements particularly at low energy section, which increases the possibility of temporary or permanent failure of accelerating cavities and focusing magnets during the operation of linac. Failure of the beam transport elements like cavity, solenoid and quadrupole alters the focusing period of the beam, resulting in a mismatch of the beam with the subsequent sections. This, in turn, causes beam losses. Sensitivity of the linac performance to failure of elements also depends on the location of the failed elements. In some cases, failure of the beam transport element results in huge beam losses. Studies are performed to analyze failure of cavity and magnets at the critical location in lattice. A local compensation method is presented to compensate failure effects.

\subsection{Assumptions and principles of lattice design}

The output beam of the Project-X linac is distributed to various experiments facilities. Thus, it is necessary to design extremely reliable linac which could produce high quality output beam in order to support requirements of all experiments simultaneously. Design 


\section{BEAM DYNAMICS STUDY OF PROJECT-X CW LINAC}

of lattice includes the design and optimization of the beam transport line which consists of principal elements such as cavities and magnets. Design of ion linac lattice is more complex than that of electron linac. Complexity of design is based on the fact that ion velocity changes during acceleration significantly and it becomes relativistic at very high energies. It results in phase slippage which not only affects acceleration efficiency in multi-cell cavities but it can also lead to the coupling between longitudinal and transverse plane through the transverse RF kick due to cavities. Experience with already existing high intensity linac such as SNS [67] and past studies have led to a set of rules [68] which helps in design of lattice for the linac of Project-X facility.

Adiabatic variation in phase advance: In high intensity ion linac, non-linear forces play a crucial role in beam stability. Thus, lattice is designed with smoothly varying longitudinal and transversing focusing. It results in gradual changes in beam size. Rapid changes in beam size can lead to rapid changes in space charge force. Keeping adiabatic variation in phase advance in all planes for zero current and avoiding any abrupt changes in beam envelopes help to minimize the impacts of non-linear forces along the linac. Although this linac will not operate at space charge dominated regime yet this approach provides a design of current-independent lattice [69].

Beam matching: The smooth envelope evolution along the linac can be obtained if beam is properly matched with beam transport channel. A beam is called matched when its Twiss parameters at a given position ' $s$ ' corresponds to Twiss parameters of beam transport channel. A beam matched to a periodic focusing system has envelope oscillation of minimum amplitude and emittance growth caused by nonlinearity of focusing magnets is minimum.

The linac with different families of multi-cell cavities is segmented into different sections. Each section has different focusing period. Thus, linac can be considered as quasi-periodic system with varying period length. Transition from one focusing section to another with different properties or transition from one cryomodule to another results in discontinuity in focusing period in lattice which can lead to halo formation and emittance growth. Thus, it is necessary to perform careful matching between two sections especially at low energy section of the linac. Matching between periodic focusing sections is obtained by adjusting the gradients and phases of the outermost elements. Minimization of beam mistmach can be obtained again by avoiding any large variation in phase advance at the both side of transition. 
Robustness of lattice design: The linac uses large number of cavities and magnets. Fabrication errors and other technical aspect may affect performance of beam line elements. Thus, it is quite possible that cavities and magnets may not operate at designed RF parameters. This results in spread of operating parameters over the length of linac. This aspect should be taken into account during lattice design. Lattice should also be capable of sustaining stable operation even in presence of failure of at least one RF cavity or focusing magnets.

Minimization of Halo formation and emittance growth: Halo formation is one of the biggest concern in high intensity linac. Halo formed at low energy results in beam losses at high energy. It also degrades beam quality and causes emittance growth. Halo minimization is one of main consideration of lattice design. Avoiding any non-adiabatic changes in lattice and proper equipartition between planes reduce halo formation.

Cryogenic losses and field limitation: Operating gradients in RF cavities are chosen in such a way that corresponding peak surface magnetic field should be below $70 \mathrm{mT}$ for high energy sections and $60 \mathrm{mT}$ for low energy sections. The corresponding peak surface electric fields are kept below $40 \mathrm{MV} / \mathrm{m}$. Solenoids can provide maximum focusing field of $6 \mathrm{~T}$ and quadrupoles can provide maximum field gradient of 10 $\mathrm{T} / \mathrm{m}$. Cryogenic losses per cavities are kept below $25 \mathrm{~W}$. It is done to achieve reliable operation.

Focusing period: In order to achieve smooth focusing to the beam, short focusing period are chosen in lattice especially at low energy section where space charge force is significant.

\subsection{SC linac architecture of Project-X}

\subsubsection{Low energy section}

Low energy section of superconducting linac comprises of three subsections SSR0, SSR1 and SSR2 which are named on the basis of family of cavities used in these subsections. Low energy section uses single spoke resonators for acceleration of beam from $2.5 \mathrm{MeV}$ to $160 \mathrm{MeV}$ and solenoids for transverse focusing. The focusing period for each subsection in low energy section is shown in Figure 4.1. 


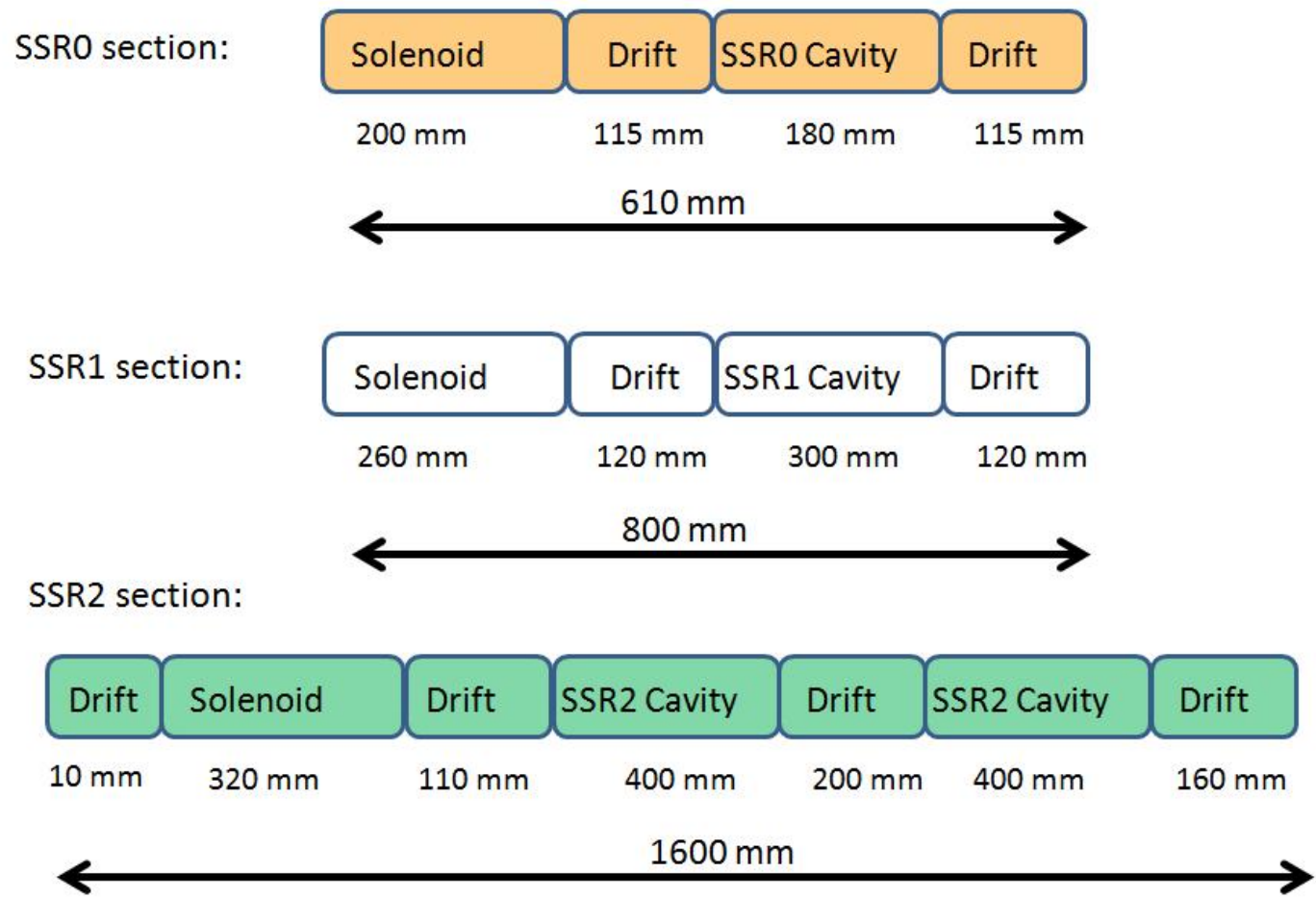

Figure 4.1: Focusing period-of SSR0, SSR1 and SSR2 subsections in low energy part of the linac.

SSR0 section: It is first superconducting subsection in low energy part of linac. It accelerates the beam coming out from RFQ. Energy of acceleration is laid in the range of $2.5 \mathrm{MeV}$ to $10.2 \mathrm{MeV}$. This section includes 18 SSR0 cavities and 18 solenoids which will be assembled in single cryomodule of length about $11 \mathrm{~m}$. SSR0 cavities are designed for $\beta_{G}=0.114$. Figure 4.2 shows expanded view of SSR0 cavity. Optimized parameters of SSR0 cavity [70] is summarized in Table 4.1

SSR1 section: SSR0 is followed by SSR1 subsection. One focusing period in SSR1 is of the length of $0.8 \mathrm{~m}$. It consists of one cavity per solenoid. There are total 10 focusing periods per cryomodule and it requires 20 cavities to cover the energy range from 10.2 MeV to 42.6 MeV. Thus, SSR1 subsection comprises of two cryomodules which are separated from each other by a warm short section of length $0.4 \mathrm{~m}$. Each cryomodule in low energy section is connected through the warm sections. It makes machine more reliable. This gap also provides an opportunity for beam collimation 


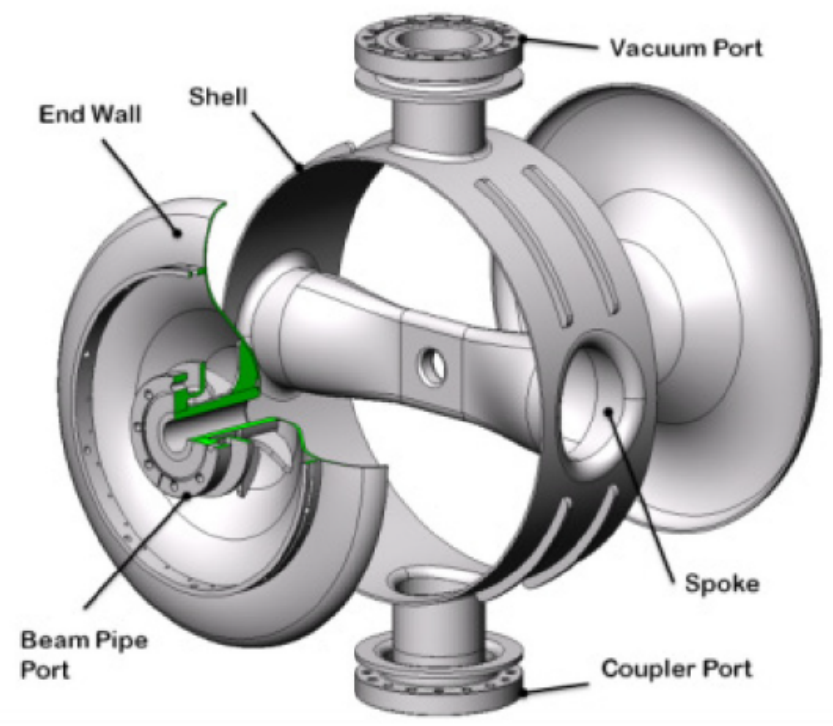

Figure 4.2: Expanded view of SSR0 cavity [1].

Table 4.1: SSR0 cavity parameters.

\begin{tabular}{ccc}
\hline Parameters & Unit & Magnitude \\
\hline Operating frequency & $\mathrm{MHz}$ & 325 \\
$\beta_{G}$ & - & 0.114 \\
$\mathrm{E}_{\text {Peak }} / \mathrm{E}_{a c c}$ & - & 5.63 \\
$\mathrm{~B}_{\text {Peak }} / \mathrm{E}_{a c c}$ & $\mathrm{mT} / \mathrm{MV} / \mathrm{m}$ & 6.92 \\
$\mathrm{G}$ & $\Omega$ & 50 \\
$\mathrm{R} / \mathrm{Q}$ & $\Omega$ & 108 \\
Aperture & $\mathrm{mm}$ & 30 \\
Cavity active length & $\mathrm{mm}$ & 105.2 \\
$\left(\mathrm{~L}_{e f f}=2 .(\beta \lambda / 2)\right)$ & & \\
\hline
\end{tabular}

and beam diagnostic after each cryomodule. Optimized parameters of SSR1 cavity is summarized in Table 4.2 ,

SSR1 cavity is designed and built for High Intensity Proton Source (HINS) project. Prototype of SSR1 cavity has been tested at Vertical Test Stand (VTS) facility. Variation in Quality factor with accelerating gradient is shown in Figure 4.3. Cavity was cooled down and tested at $2 \mathrm{~K}$ and $4 \mathrm{~K}$. Maximum accelerating gradient achieved after crossing multipacting barrier is $33 \mathrm{MV} / \mathrm{m}$ and $25 \mathrm{MV} / \mathrm{m}$ at $2 \mathrm{~K}$ and $4 \mathrm{~K}$ respectively. 
Table 4.2: SSR1 cavity parameters.

\begin{tabular}{ccc}
\hline Parameters & Unit & Magnitude \\
\hline Operating frequency & $\mathrm{MHz}$ & 325 \\
$\beta_{G}$ & - & 0.215 \\
$\mathrm{E}_{\text {Peak }} / \mathrm{E}_{a c c}$ & - & 3.84 \\
$\mathrm{~B}_{\text {Peak }} / \mathrm{E}_{a c c}$ & $\mathrm{mT} / \mathrm{MV} / \mathrm{m}$ & 5.81 \\
$\mathrm{G}$ & $\Omega$ & 84 \\
$\mathrm{R} / \mathrm{Q}$ & $\Omega$ & 242 \\
Aperture & $\mathrm{mm}$ & 30 \\
Cavity active length & $\mathrm{mm}$ & 198.5 \\
$\left(\mathrm{~L}_{\text {eff }}=2 .(\beta \lambda / 2)\right)$ & & \\
\hline
\end{tabular}

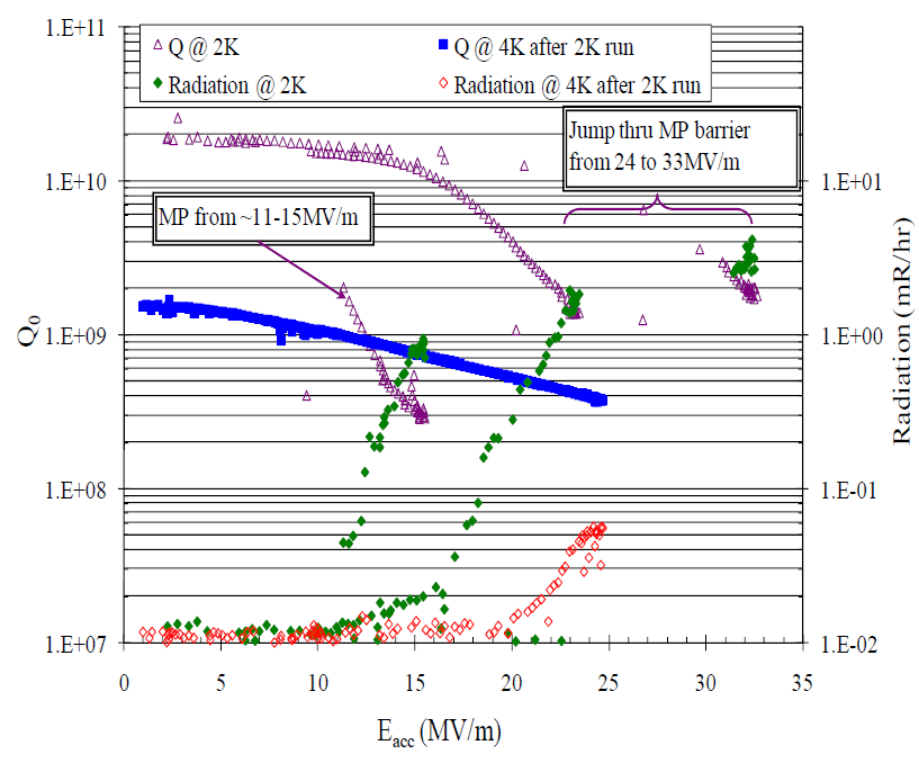

Figure 4.3: $\mathrm{Q}_{0}$ vs $\mathrm{E}_{a c c}$ results of bare SSR1 cavity in VTS. Blue curve is at $4 \mathrm{~K}$ and pink curve is at $2 \mathrm{~K} .70$.

SSR2 section: SSR1 is followed by SSR2 subsection. It comprises of four cryomodules and each cryomodule consists of 10 SSR2 cavities. Each focusing period in SSR2 subsection consists of two SSR2 cavities per solenoid. This subsection covers the energy range from $42.6 \mathrm{MeV}$ to $160 \mathrm{MeV}$. Optimized parameters of SSR2 cavity is summarized in Table 4.3 , 
Table 4.3: SSR2 cavity parameters.

\begin{tabular}{ccc}
\hline Parameters & Unit & Magnitude \\
\hline Operating frequency & $\mathrm{MHz}$ & 325 \\
$\beta_{G}$ & - & 0.419 \\
$\mathrm{E}_{\text {Peak }} / \mathrm{E}_{a c c}$ & - & 3.67 \\
$\mathrm{~B}_{\text {Peak }} / \mathrm{E}_{a c c}$ & $\mathrm{mT} / \mathrm{MV} / \mathrm{m}$ & 6.93 \\
$\mathrm{G}$ & $\Omega$ & 109 \\
$\mathrm{R} / \mathrm{Q}$ & $\Omega$ & 292 \\
Aperture & $\mathrm{mm}$ & 40 \\
Cavity active length & $\mathrm{mm}$ & 386.5 \\
$\left(\mathrm{~L}_{\text {eff }}=2 .(\beta \lambda / 2)\right)$ & & \\
\hline
\end{tabular}

\subsubsection{Intermediate and High energy sections}

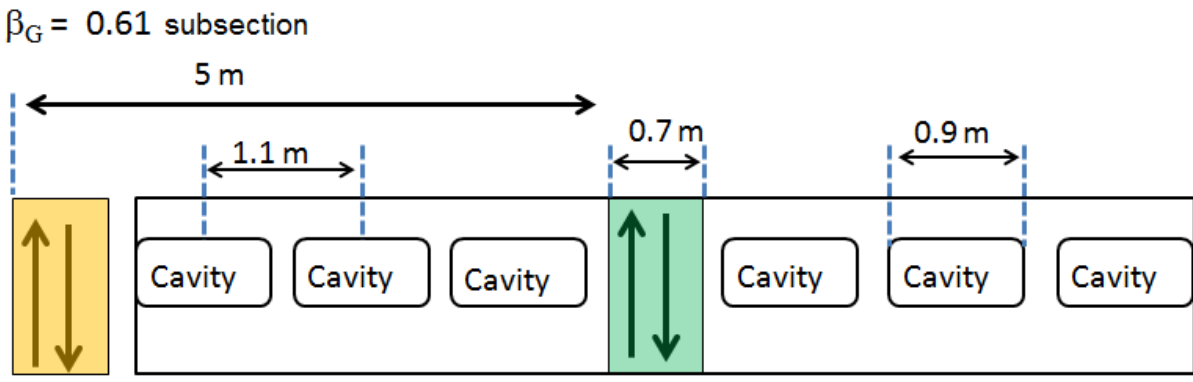

NC Doublet

SC Doublet

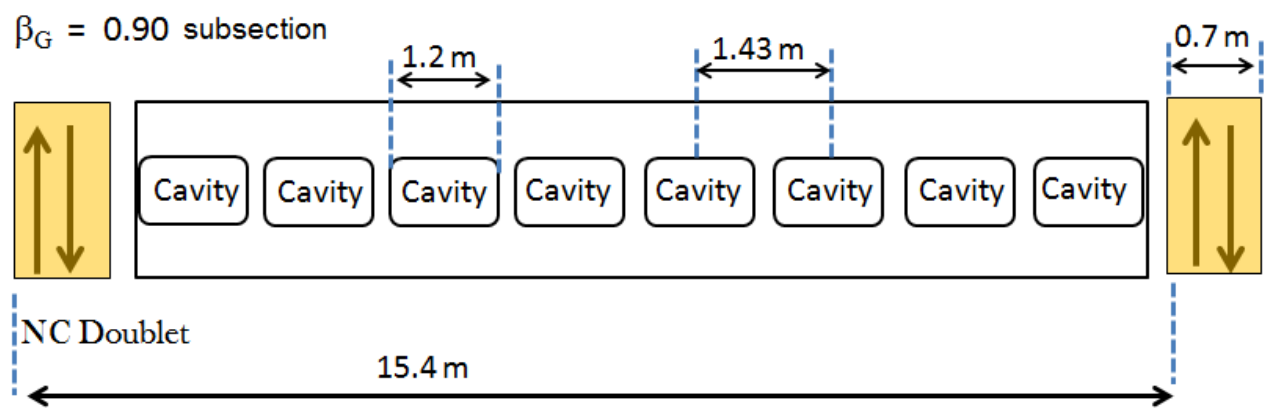

Figure 4.4: Focusing period-of $\beta_{G}=0.61$ and $\beta_{G}=0.90$ subsections in intermediate and high energy part of linac.

High energy section is made of two subsections $\beta_{G}=0.61$ and $\beta_{G}=0.90$ which are named on the basis of geometrical beta of cavities used in these sections. Layout of 


\section{BEAM DYNAMICS STUDY OF PROJECT-X CW LINAC}

focusing schemes in $\beta_{G}=0.61$ and $\beta_{G}=0.90$ subsections are shown in Figure 4.4 .

$\beta_{G}=\mathbf{0 . 6 1}$ subsection: This subsection accelerates the beam from energy $160 \mathrm{MeV}$ to $515 \mathrm{MeV}$. It requires six cryomodules of $\beta_{G}=0.61,5$-cell, $650 \mathrm{MHz}$ cavities to cover up the given range of acceleration. Each cryomodules consists of six cavities and there is one focusing doublet for every three cavities. It should also be noted that there are alternative arrangement of normal conducting and superconducting doublet for every second focusing period.

$\beta_{G}=\mathbf{0 . 9 0}$ subsection: This subsection accelerates the beam from energy $515 \mathrm{MeV}$ to $3 \mathrm{GeV}$. It requires nineteen cryomodules of $\beta_{G}=0.90,5$-cell, $650 \mathrm{MHz}$ cavities to cover up the given range of acceleration. Each cryomodules consists of eight cavities and there is one focusing doublet for every eight cavities. Number of focusing elements and RF cavities in each subsections along with transition energy and focusing period are summarized in Table 4.4 .

Table 4.4: Number of elements in each section along with transition energy.

\begin{tabular}{cccccc}
\hline & SSR0 & SSR1 & SSR2 & $\beta_{G}=\mathbf{0 . 6 1}$ & $\beta_{G}=\mathbf{0 . 9 0}$ \\
\hline Cavities & 18 & 20 & 40 & 36 & 152 \\
Solenoids & 18 & 20 & 20 & 0 & 0 \\
Quads & 0 & 0 & 0 & 24 & 38 \\
Cryomodules & 1 & 2 & 4 & 6 & 19 \\
Focusing period & 0.61 & 0.80 & 1.60 & 5.0 & 15.4 \\
Section length & 10.98 & 16.40 & 33.20 & 60.0 & 292.60 \\
Transition energy & 10.18 & 42.58 & 160.5 & 515.4 & 3028.3 \\
\hline
\end{tabular}

\subsection{Beam optics for baseline design of the Project- $\mathrm{X}$ linac}

Baseline lattice has been designed using described principles and constraints. Figure $4.5 \mathrm{a}$ and Figure $4.5 \mathrm{~b}$ show phase advance and phase advance per unit length respectively for transverse and longitudinal oscillation along the linac for zero current. As from our principle requirements, these curves should be smooth in high intensity current machine. It can be seen that curves are smooth but one can notice irregular jumps and discontinuities which arise at transition due to beam matching. These discontinuities are minimized by performing smooth beam matching. Gradients and voltage gain 


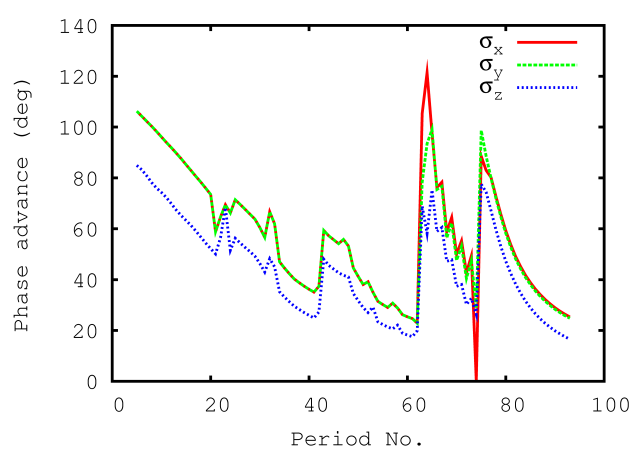

(a)

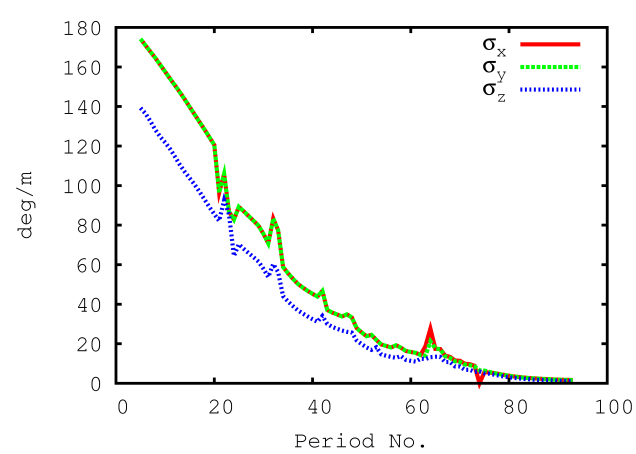

(b)

Figure 4.5: Longitudinal and transverse (a) phase advance and (b) phase advance per unit length along the linac for zero current.

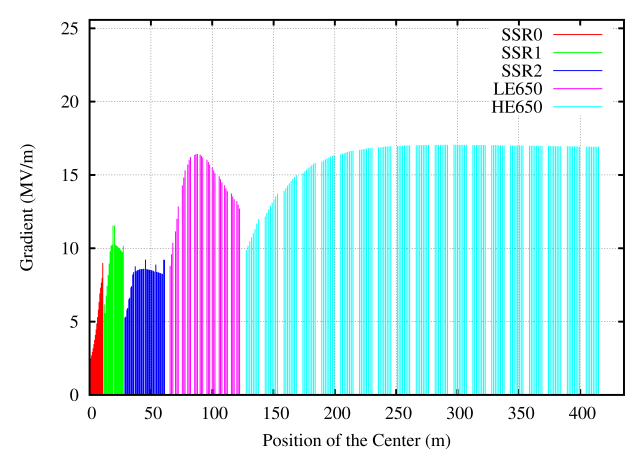

(a)

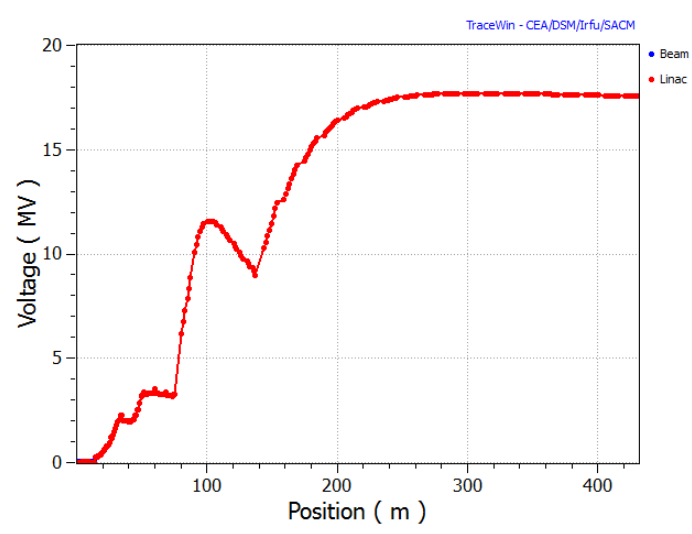

(b)

Figure 4.6: (a) Distribution of gradient and (b) voltage gain in cavities along the linac.

per cavity along the linac are shown in Figures $4.6 \mathrm{a}$ and $4.6 \mathrm{~b}$ respectively. The axial magnetic field of solenoids in SSR0, SSR1 and SSR2 sections and quadrupole gradients in intermediate and high energy sections of the linac are shown in Figures $4.7 \mathrm{a}$ and $4.7 \mathrm{~b}$ respectively.

Studies are performed to analyze the performance of baseline design of CW linac. Multiparticle simulation is performed using $3 \sigma$ Gaussian distribution of $100 \mathrm{~K}$ macroparticles. Figure $4.8 \mathrm{a}$ shows $1 \sigma_{r m s}$ horizontal (x) beam envelope in positive axis (red) and 


\section{BEAM DYNAMICS STUDY OF PROJECT-X CW LINAC}

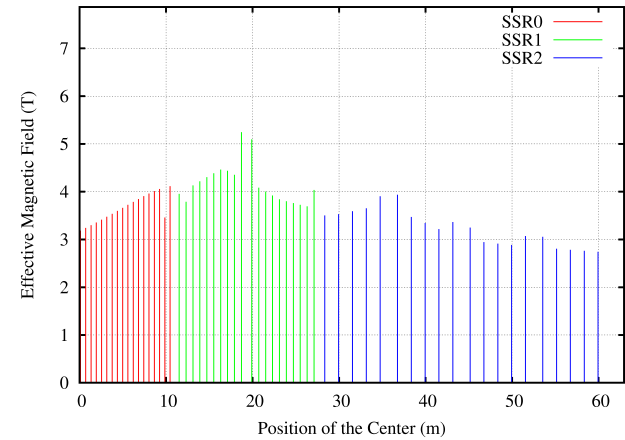

(a)

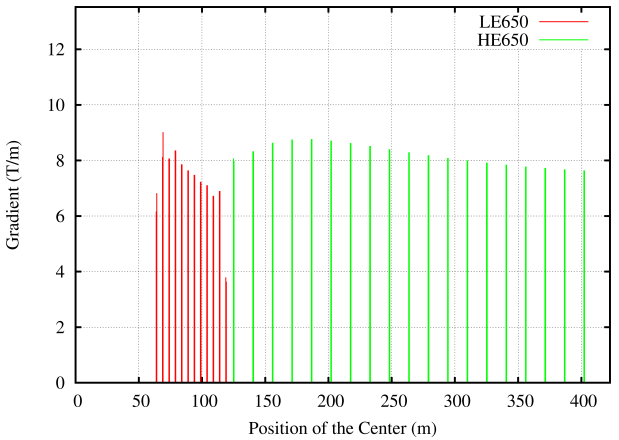

(b)

Figure 4.7: (a) Axial magnetic field in solenoids in SSR0, SSR1 and SSR2 sections (b) quadrupole gradients in intermediate and high energy section of the linac.

$1 \sigma_{r m s}$ vertical beam envelope (y) in negative axis (blue). Figure $4.8 \mathrm{~b}$ shows longitudinal beam size which is plotted for $-1 \sigma_{r m s}$ and $1 \sigma_{r m s}$ beam along the linac. Evolution of beam emittance in longitudinal (green) and transverse (red) planes through the linac are shown in Figure 4.8c. It can be noticed that emittance growth is very smooth and there is no abrupt changes in emittance growth along the linac. Initial and final emittances with beam energy are summarized in Table 4.5. To measure the magnitude of beam halo in $3 \mathrm{GeV} \mathrm{SC} \mathrm{linac,} \mathrm{halo} \mathrm{parameters} \mathrm{[52]} \mathrm{in} \mathrm{longitudinal} \mathrm{and} \mathrm{transverse}$ planes are calculated. Figure $4.8 \mathrm{~d}$ shows behavior of halo parameters along the linac. It should be accounted that halo parameters for ideal Gaussian distribution is one and hence their growth are minimized and aimed to keep around one.

Table 4.5: Beam parameters for the baseline design of the linac.

\begin{tabular}{cccc}
\hline Parameters & Unit & Start & End \\
\hline$\epsilon_{z}$ & $\pi \mathrm{mm} \mathrm{mrad}$ & 0.127 & 0.18 \\
$\epsilon_{t}$ & $\pi \mathrm{mm} \mathrm{mrad}$ & 0.250 & 0.258 \\
Energy & $\mathrm{MeV}$ & 2.517 & 3028.32 \\
\hline
\end{tabular}




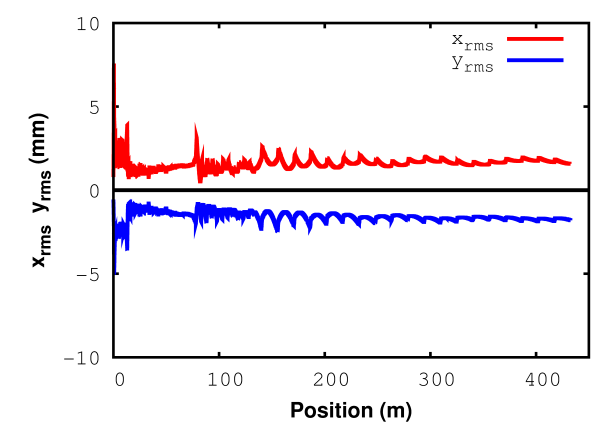

(a)

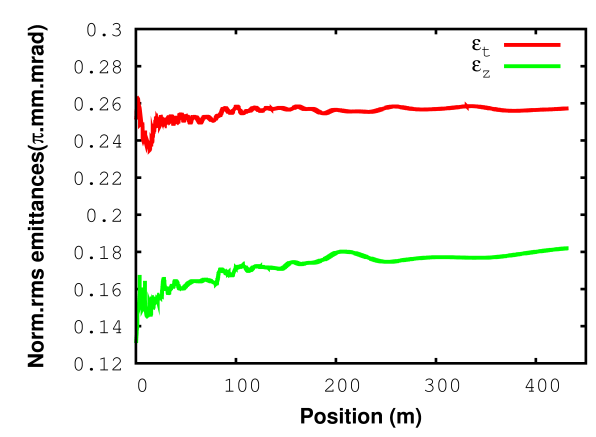

(c)

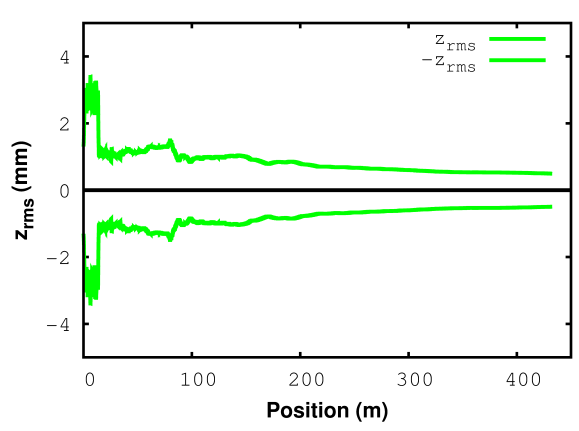

(b)

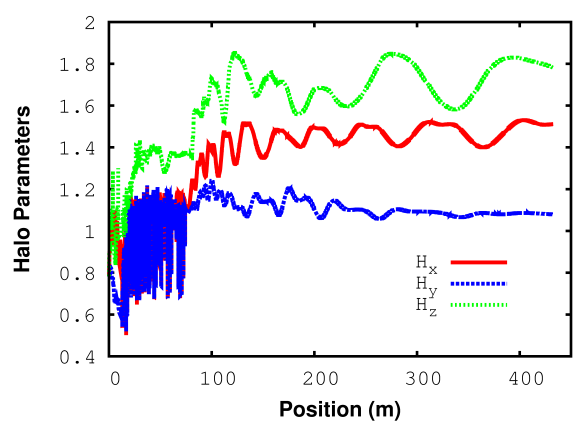

(d)

Figure 4.8: Beam parameters for baseline design (a) Transverse beam envelope, (b) longitudinal beam envelope, (c) longitudinal (green) and transverse (red) normalized emittance and (d) beam halo parameters along the linac.

\subsection{Acceptance of the linac}

In high intensity linac, acceptance parameter is a vital mean to measure linac performance. It is determined by the largest possible beam size which can be transmitted through linac without any beam losses. Thus large acceptance corresponds to high tolerance against errors. In order to determine longitudinal and transverse acceptance of a linac, simulation is performed. An input distribution is generated with relatively very large emittance than nominal beam emittance in one plane and relatively smaller in other planes. For instance, to determine longitudinal acceptance, beam distribution is generated which occupies large longitudinal phase space but very small transverse 


\section{BEAM DYNAMICS STUDY OF PROJECT-X CW LINAC}

phase space. Distribution is tracked through lattice and initial coordinates of particles at the end of linac are plotted. Figures $4.9 \mathrm{a}$ and $4.9 \mathrm{~b}$ show acceptance in longitudinal and transverse plane. Area occupied by initial coordinates of transmitted particles trace acceptance of linac for corresponding plane. Space charge effects are not included in acceptance calculation but its impact is expected like a perturbation because tune depression is not high [72. Initial coordinates of particles in phase space i.e., energy spread $(\Delta W)$ and time spread $(\Delta t)$ for longitudinal phase space and transverse position $(y)$ and transverse divergence $\left(y^{\prime}\right)$ in transverse phase space, which are transmitted through the linac, trace the acceptance boundary (green) on input distribution (red).

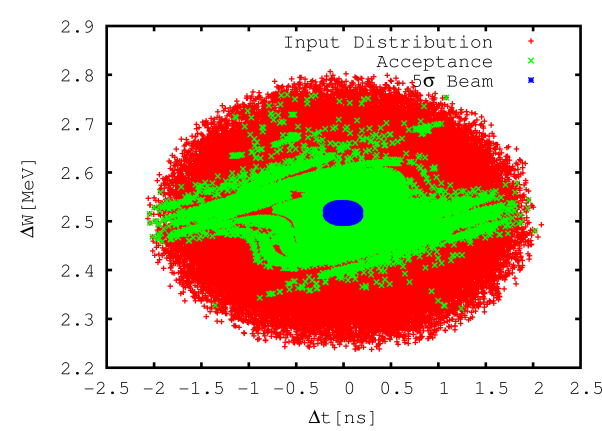

(a)

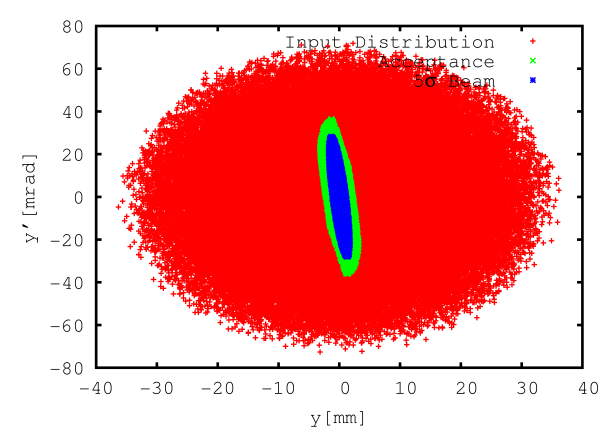

(b)

Figure 4.9: Acceptances (green) of the baseline linac in (a) longitudinal plane (b) transverse plane with $5 \sigma$ beam emittance (blue).

Area occupied by green region in Figures $4.9 \mathrm{a}$ and $4.9 \mathrm{~b}$ define acceptance of linac. Front end of linac limits the acceptance both in transverse and longitudinal plane. Although requirement of input beam distribution is $3 \sigma$ for Project-X CW linac, acceptances for baseline design are large enough to enclose $5 \sigma$ Gaussian beam distribution. Initial $5 \sigma$ Gaussian beam distribution (blue) is also shown in Figures 4.9a and 4.9b. The beam is enclosed well within the acceptance, thus no beam losses have been observed for nominal operation of linac. Particles which fall out of acceptance i.e., green region in Figures $4.9 \mathrm{a}$ and $4.9 \mathrm{~b}$ are no longer accelerated in linac and these are not matched with downstream magnetic focusing elements which are designed for fully accelerated particles and consequently these are lost in linac. 


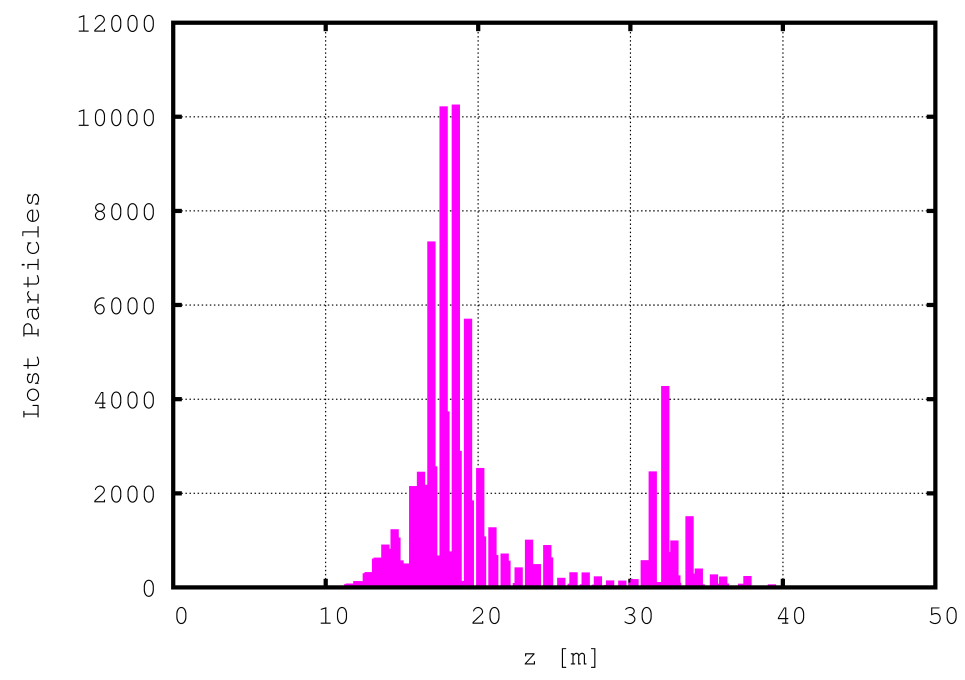

Figure 4.10: Distribution of lost particles in the linac during longitudinal acceptance calculation.

Linac is segmented into five sections and transition from one section to another may result in shrinkage of acceptance if these are not matched properly. In order to determine most sensitive section which limits the longitudinal acceptance of linac, distributions of lost particles are plotted along the length of linac in Figure 4.10. It can be seen that major portion of particle losses happen at transition between SSR0-SSR1 sections. No beam losses were observed after $40 \mathrm{~m}$ tracking through the superconducting linac. It can be concluded that longitudinal acceptance is mainly limited after SSR1 section and the result out acceptance is propagated through rest of linac. Thus, to analyze the nature of longitudinal acceptance at transition between other sections, studies are performed for transition between successive sections. Figure 4.11 shows longitudinal acceptance for different cases of transition between neighbouring sections. Figure 4.11a shows longitudinal acceptance (green) when initial distribution (red) is generated at the beginning of SSR0 and is tracked through SSR0 to the end of SSR1 section. To analyze the longitudinal acceptance between SSR1 and SSR2 section, initial distribution is generated at the beginning of SSR1 section and tracked up to the end of SSR2 section (Figure 4.11b). Similar approach is used to determine longitudinal acceptance for SSR2 to $\beta_{G}=0.61$ (Figure 4.11c) and $\beta_{G}=0.61$ to $\beta_{G}=0.90$ (Figure 4.11d) 


\section{BEAM DYNAMICS STUDY OF PROJECT-X CW LINAC}

sections. It should be noted that energy spread (y-axis) in Figure 4.11 are different due to different beam energies at the beginning of the respective sections. It can be

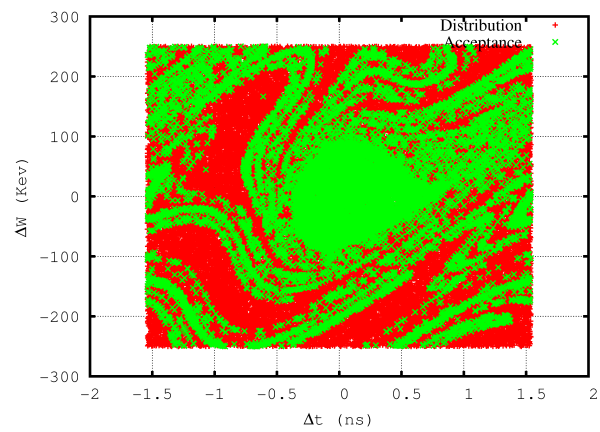

(a)

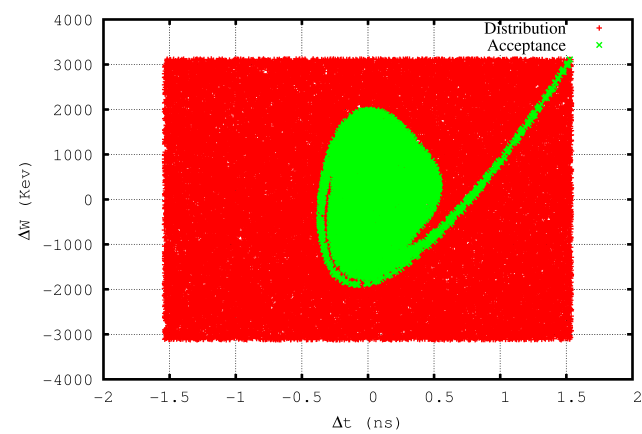

(c)

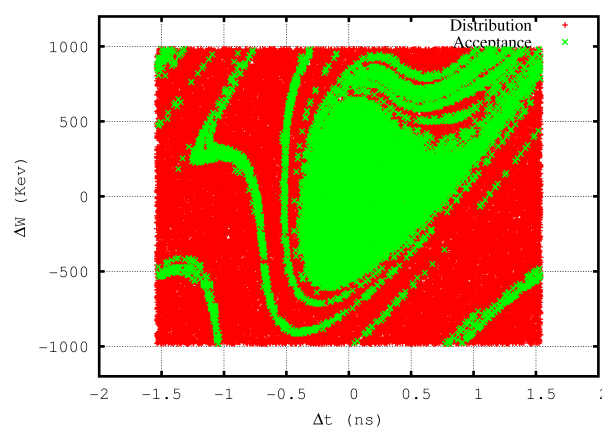

(b)

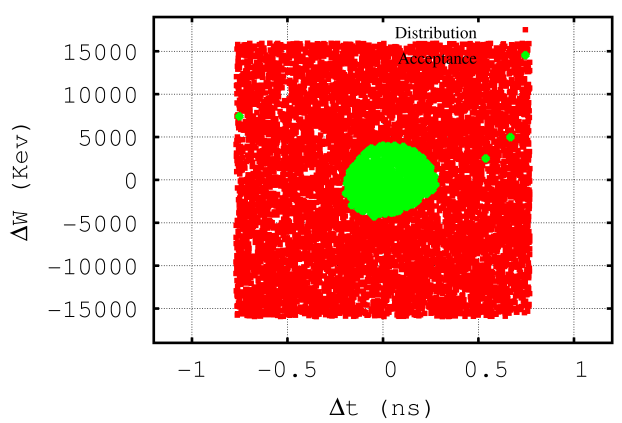

(d)

Figure 4.11: Longitudinal acceptance through transition between (a) SSR0 and SSR1 (b) SSR1 and SSR2 (c) SSR2 and $\beta_{G}=0.61$ and (d) $\beta_{G}=0.61$ and $\beta_{G}=0.90$ sections (scales are different in different plots).

observed from Figure 4.11 that transition between SSR0 to SSR1 section is critical one which limits longitudinal acceptance of linac. Thus, in order to improve longitudinal acceptance of linac, matching between these two sections can be improved by choosing appropriate phases and gradients in cavities although longitudinal acceptance is large enough to enclose $5 \sigma$ beam as shown in Figure $4.9 \mathrm{a}$. 


\subsection{Reliability of the linac}

An essential measure of a successful accelerator system is its ability to provide high beam availability and high reliability. The multiuser facility and further upgrade for higher current to test Project-X as a test facility of accelerator driven system (ADS) requires high beam availability and hence reliability of the linac. Thus, possibility of failure of beam line elements are included in lattice design. Operation of the SC linac at $\mathrm{CW}$ mode puts stringent tolerances on beam transport elements, especially at low energy sections, which increase possibility of temporary or permanent loss of accelerating cavities and focusing magnets during the operation of linac. Failure of the beam transport elements like cavity, solenoid and quadrupole alters the focusing period of the beam, resulting in a mismatch of the beam with the subsequent sections. This, in turn, causes beam losses. Sensitivity of the linac performance towards failure's effects also depends on the location of failed elements. In some cases, failure of the beam transport element results in huge beam losses and it becomes necessary to replace this element for nominal operation of the machine. To recover nominal performance of linac using traditional way comprises replacing of a complete cryomodule. It is required to warm up cryomodule from operating temperature (usually $2 \mathrm{~K}$ ) to room temperature and after replacing; cryomodule is cooled down from room temperature to operating temperature. Furthermore, procedure to resume the nominal operation is identical to starting procedure and requires slowly ramping up in beam. Thus, these beam interruptions reduce the beam availability for the different experiments for the long time. Lattice design should be robust enough to have a capability that RF cavity or magnet failure may be compensated locally by using the neighbouring elements. The numbers of cavities and focusing magnets in a cryomodule are chosen in such a way that at least one failure of RF cavity or focusing magnet can be compensated locally. Cryomodules in low energy sections are segmented and hence transitional beam energy (one cryomodule to another) is chosen very carefully. In the absence of careful design of beam transition, beam matching with subsequent section becomes very sensitive to errors and it becomes very difficult to retune the linac in presence of failed elements. 


\section{BEAM DYNAMICS STUDY OF PROJECT-X CW LINAC}

\subsubsection{Failure of RF elements}

There are lot of scenario which may affect nominal performance of beam line elements. These scenario can result in temporary or permanent loss of RF cavities and magnets in beam transport line. Temporary failures are those which have minimal impact and can be corrected by applying appropriate method such as sudden changes in resonance frequency because of mechanical stress or helium pressure fluctuation. Resonance frequency can be corrected by phase loop. Situations which cause significant degradation in performance of RF cavity and can result in its permanent loss in beam transport lines are reviewed below:

- Failure of cavity tuner: the role of tuner is to ensure that cavity resonance frequency matches with designed operating frequency. In the absence of tuner, cavity will be out of resonance regime and thus it will be unavailable for beam acceleration.

- Failure of power coupler due to window problem, multipacting, cooling, high power dissipation etc.

- Failure of RF power supply: Failure of elements like klystron, circulator, divider etc. in power distribution line results in interruption of RF power supply to cavity.

- Degradation in inner surface of RF cavity during operation causes field emission which induces excessive drop in quality factor (Q) of cavity and thus results in increase in cryogenic losses. It can reduce cavity field below an acceptable level which results in mismatch of beam energy with subsequent sections.

The failure of superconducting magnets in lattice can happen due to breakage of conducting coil, electrical insulation breakdown, possible quench, interruption in power supply, etc. In following section we discuss the most critical cases of failure of beam line elements in lattice and their compensation using local compensation scheme.

\subsubsection{Local compensation scheme}

Analysis is performed to compensate the effects of failure of beam line elements with minimal user disruption. Neighbouring elements in the vicinity of failed element are 
retuned in order to achieve designed beam energy at the end of particular section and smooth beam propagation through the linac. Separate power supply for each cavity allows one to set the RF phases and amplitudes in cavities independently. Thus, RF phase and field amplitude of RF cavities are varied to recover beam energy and to achieve smooth longitudinal profile of beam while field gradient of the solenoid and quadrupole are changed to tune transverse dynamics. The constraints and assumptions which are applied during local compensation are summarized below:

- Accelerating field in cavity: accelerating fields are increased to recover the beam energy but it is ensured that corresponding surface peak magnetic field in cavity should not exceed above $70 \mathrm{mT}$.

- Synchronous phases $\left(\phi_{\text {synch }}\right)$ are varied in order to provide longitudinal focusing and acceleration to beam. Synchronous phase also determine RF bucket (separatrix) size available for particles for stable acceleration. Thus, synchronous phase are varied in such a way that ratio of synchronous phase to longitudinal beam size $\left(\phi_{\text {beam }}=(\omega z) / \beta c\right)$ should be greater than 3 . It is required to achieve initial operational requirement of transmission of $3 \phi$ beam through the lattice.

$$
\frac{\phi_{\text {synch }}}{\phi_{\text {beam }}} \geq 3
$$

- Gradient in magnetic elements: change in accelerating field and synchronous phase of cavities also change the magnitude of radial kick due to cavities and thus, it alters transverse focusing period. Magnets and cavities are also retuned accordingly to match the beam in transverse plane.

- Magnetic field gradient in quadrupole and axial magnetic field in solenoids should not exceed above design limit which is $10 \mathrm{~T} / \mathrm{m}$ and $6 \mathrm{~T}$ for quadrupole and solenoid respectively.

- Minimal user disruption: minimization of number of retuned elements is necessary to expedite the process of compensation. 


\section{BEAM DYNAMICS STUDY OF PROJECT-X CW LINAC}

\subsubsection{Failure of RF cavity}

Studies have been performed in detail to analyze the effects of RF cavity failures at critical locations in beam transport line, especially in the low energy section of the SC linac. Cavity failure results in significant deformation in the longitudinal phase space, which in turn induces strong envelope oscillations and halo formation.

\subsubsection{Study and local compensation of failure of first cavity in SSR0 section}

Study of failure of first cavity in SSR0 section: Since SSR0 is first superconducting section in CW linac, initial beam energy at front end of SSR0 section is only 2.517 $\mathrm{MeV}$. Beam is non-relativistic and space charge effects are dominant at this energy. As shown in Figure 4.1 one period in SSR0 section consists of one solenoid and one cavity. Failure of first cavity in SSR0 section introduces extra drift space which results in delay of first accelerating kick and hence energy gain for beam. Beam size in longitudinal plane starts to grow up due to mismatch with subsequent sections. It results in emittance growth and beam halo formation at low energy section which leads to beam losses at high energy section. R.m.s. beam envelope in $x, y$ and $z$ planes, emittance dilution, and longitudinal halo parameter along the linac are shown in Figures 4.12a, $4.12 \mathrm{~b}, 4.12 \mathrm{c}$ and $4.12 \mathrm{~d}$ respectively, for the case when first cavity in SSR0 section is failed. It can be seen (Figure 4.12b) that failure of first cavity results in significant distortion of longitudinal profile of the beam which causes emittance growth and beam losses. Beam losses happen at higher energy sections mainly at transition between SSR2 to $\beta_{G}=0.61$ section where frequency jump (325 MHz to $650 \mathrm{MHz}$ ) is made in linac and also at the transition between $\beta_{G}=0.61$ to $\beta_{G}=0.9$ section. It can also be noticed that there is minimal impact of RF cavity failure on transverse beam profile.

Failure of RF cavity also affects acceptance of linac. Failure of first cavity in SSR0 section reduces longitudinal acceptance of linac however transverse acceptance have minimal impact. Figure 4.13a shows deformed longitudinal acceptance (red) with $5 \sigma$ beam distribution (green). The beam distribution touches acceptance boundary in longitudinal plane. It may be concluded that further mismatch in longitudinal plane place a big fraction of beam out of accepted region which results in significant beam losses. Thus, it is necessary to compensate failure effects of RF cavity to restore longitudinal 


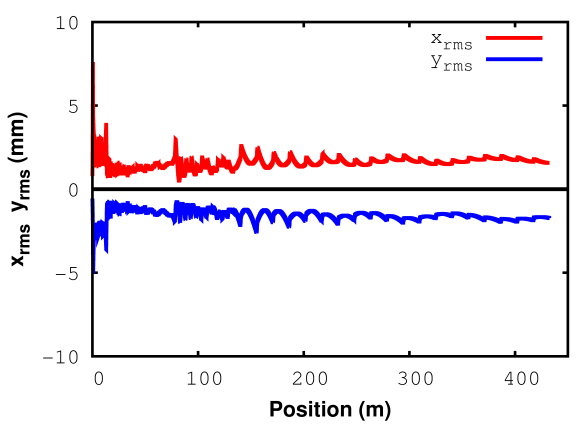

(a)

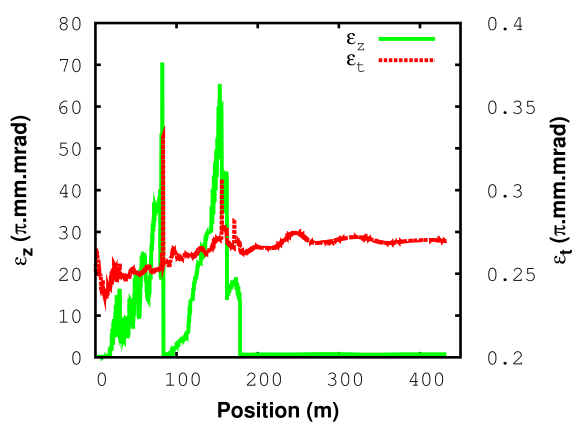

(c)

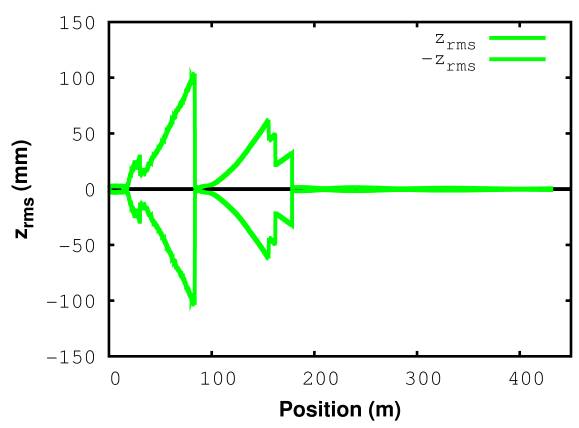

(b)

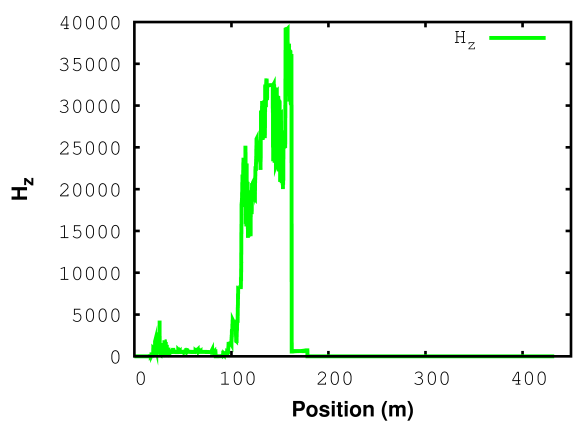

(d)

Figure 4.12: Beam envelope in (a) transverse plane (b) longitudinal plane (c) beam normalized rms emittances and (d) longitudinal halo parameter along the linac after failure of first cavity in SSR0 section.

acceptance and hence sensitivity of linac. Figure $4.13 \mathrm{~b}$ shows transverse acceptance (red) with $5 \sigma$ beam distribution (green).

Local Compensation of failure of first cavity in SSR0 section: The local compensation scheme is applied and neighbouring elements are retuned to restore same beam energy at the end of SSR0 section so that design beam parameters at the end of SSR0 section is recovered. Numbers of retuned elements and their location relative to failed cavity are shown in Figure 4.14. Two cavities which are referred as gaps (RF gaps are analytical substitution of RF cavity), two solenoids and a quad triplet in the upstream MEBT normal conducting section, one solenoid in the same period of the 


\section{BEAM DYNAMICS STUDY OF PROJECT-X CW LINAC}

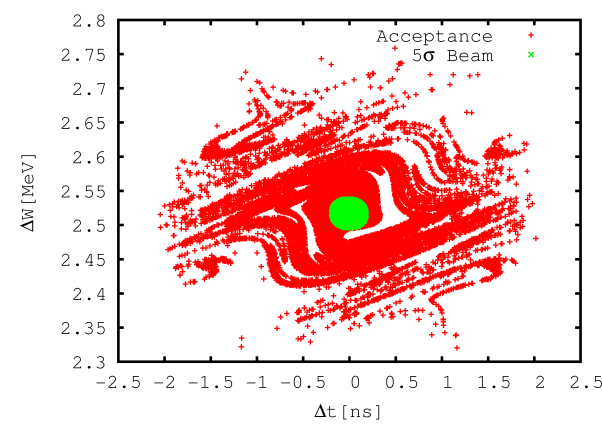

(a)

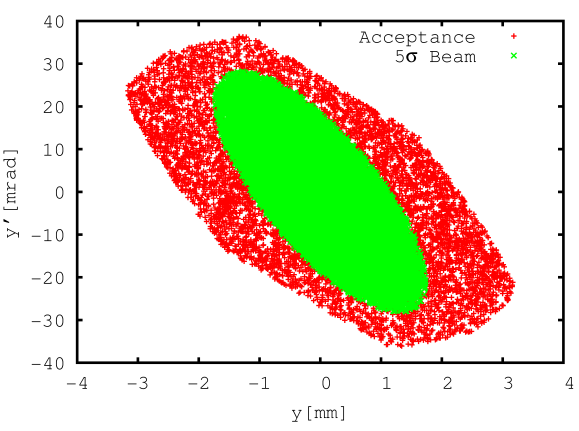

(b)

Figure 4.13: (a) Longitudinal acceptance and (b) transverse acceptance with $5 \sigma$ beam distribution (green) after failure of first cavity in SSR0 section.

failed cavity, and one solenoid and one cavity in each of the three downstream periods (after the failed cavity) are used to retune the lattice for smooth beam propagation.

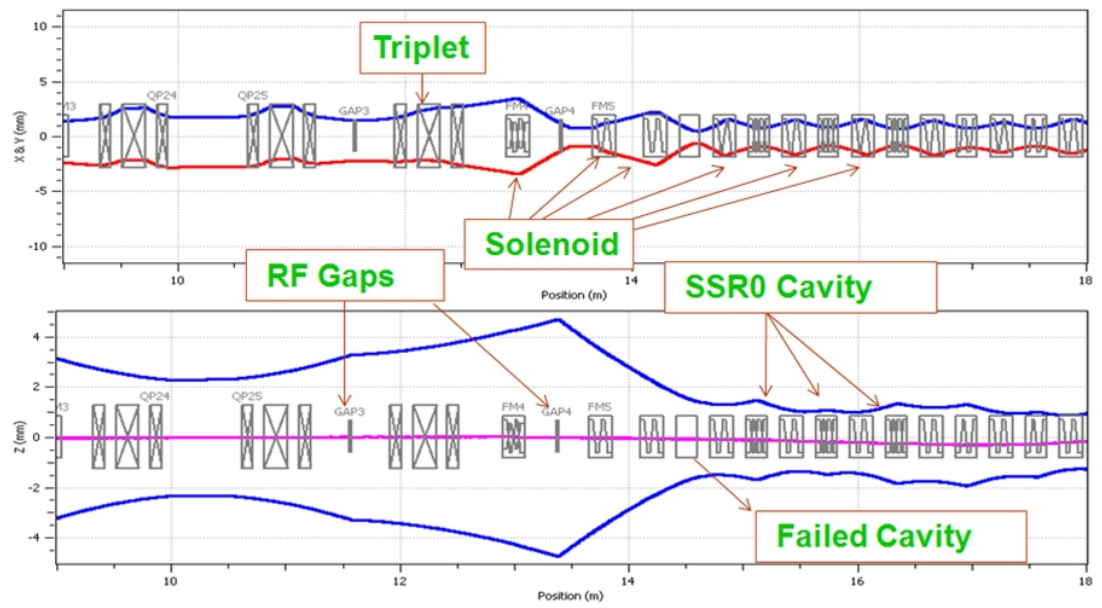

Figure 4.14: Enlarged view of beam profile in transverse plane (top) and longitudinal plane (bottom) in presence of a failed cavity after its compensation with retuned elements.

Beam profile along the linac after applying compensation is studied. It can be seen in Figure 4.15a that longitudinal beam profile is restored and one can see there is no beam losses after applying local compensation. There is $100 \%$ beam is transmitted through the linac. There is almost no impact of local compensation scheme on the transverse profile of the beam in this case. Figure $4.15 \mathrm{~b}$ shows transverse trajectories 
of beam. Beam transverse and longitudinal emittance are also calculated. It can be

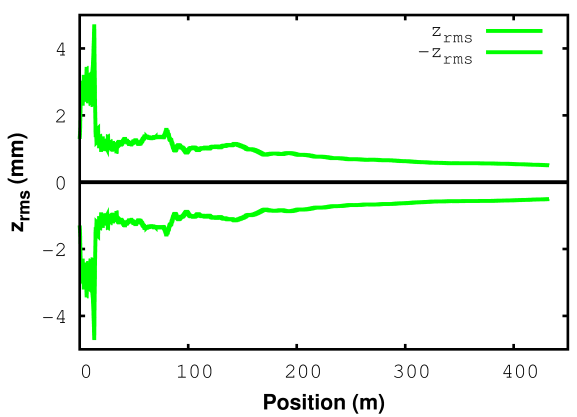

(a)

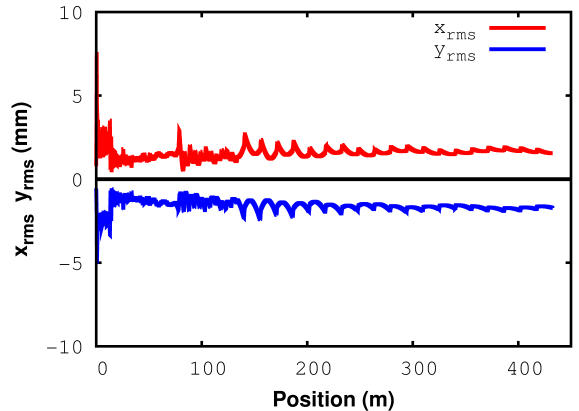

(b)

Figure 4.15: Beam profile in (a) transverse plane and (b) longitudinal plane in presence of a failed cavity after its compensation.

observed from Figure 4.16a that longitudinal emittance is restored to large extent. One can also see the improvement in transverse emittance. Figure $4.16 \mathrm{~b}$ shows longitudinal beam halo parameters. There is a significant improvement in beam halo parameter as compared to Figure $4.12 \mathrm{~d}$, Figure $4.16 \mathrm{c}$ shows beam longitudinal size in degree for $1 \sigma$ and $3 \sigma$ beam with synchronous phases in each cavity after local compensation of failed cavity. It can be noticed that synchronous phases are bigger than $3 \sigma$ beam size which accomplishes the required condition given by equation (4.1) and ensures smooth transition of beam in longitudinal plane along the linac.

Longitudinal acceptance is also restored after applying local compensation scheme. Restored acceptances look similar to the nominal acceptance. Important beam parameters are summarized in Table 4.6.

\subsubsection{Study and local compensation of failure of last cavity in SSR0 sec- tion}

SSR0 and SSR1 cryomodules are separated by $400 \mathrm{~mm}$ warm gap. Fields and phases are adjusted in outermost elements of both side of gap to achieve smooth transition of beam and hence avoiding emittance growth and halo formation during beam propagation from one section to another. Thus, failure of last element, either cavity or magnet, is very critical as it destroys matched condition at transition and results in beam mismatch 


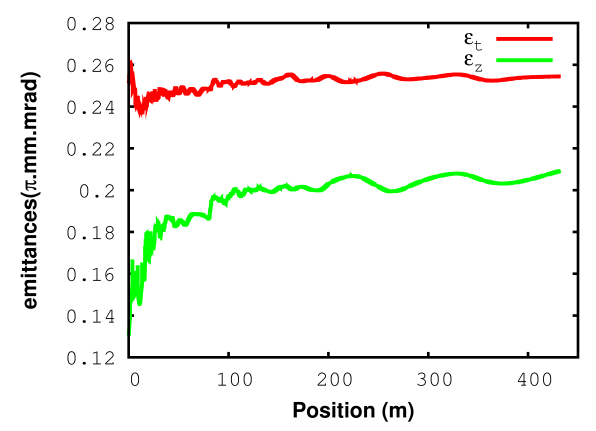

(a)

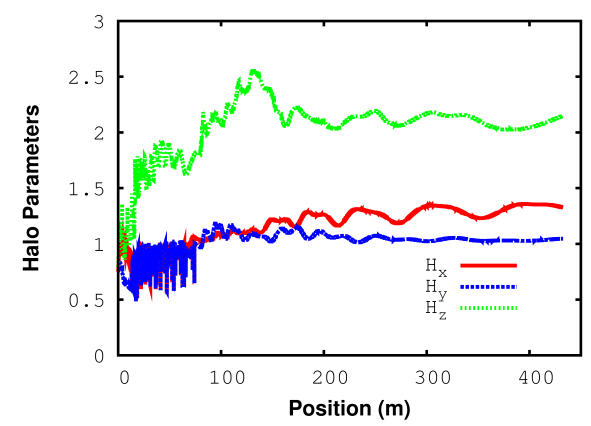

(b)

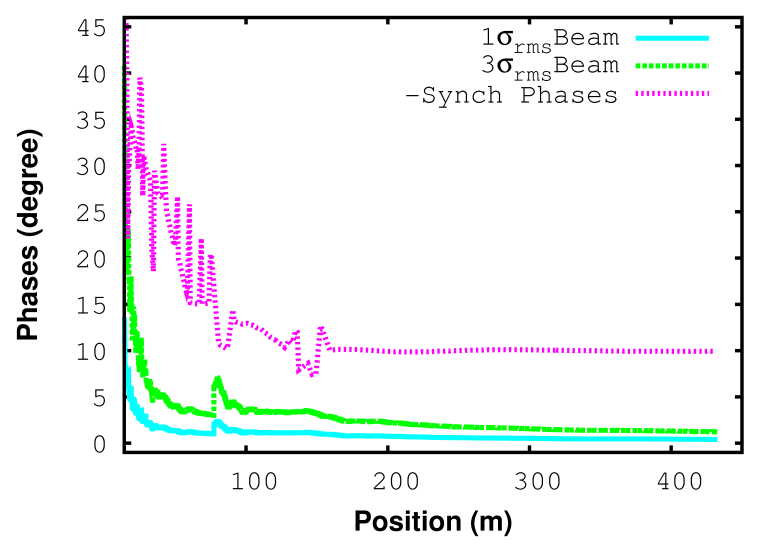

(c)

Figure 4.16: Beam quality parameters after applying local compensation scheme. (a) Longitudinal and transverse emittance. (b) Halo parameters. (c) Beam longitudinal size for $1 \sigma$ and $3 \sigma$ beam with synchronous phase.

with subsequent sections. It becomes further crucial if it happens at low energy part of the linac. Study is performed to understand the effects of failure of last cavity in SSR0 section.

Figure 4.17 shows enlarged view of lattice period with the failed cavity in SSR0 section. It can be noticed from Figure 4.17 that after failure of last cavity in SSR0 section, beam propagates about $1.5 \mathrm{~m}$ without any acceleration which alters beam longitudinal focusing period and hence results in mismatch in longitudinal plane while 
Table 4.6: Beam parameters at the end of linac.

\begin{tabular}{|c|c|c|c|c|}
\hline \multirow[t]{2}{*}{ Parameters } & \multirow[t]{2}{*}{ Unit } & \multirow[t]{2}{*}{ Nominal lattice } & \multicolumn{2}{|c|}{$\begin{array}{l}\text { Lattice with failure } \\
\text { of first SSR0 cavity }\end{array}$} \\
\hline & & & $\begin{array}{c}\text { Before } \\
\text { compensation }\end{array}$ & $\begin{array}{c}\text { After } \\
\text { compensation }\end{array}$ \\
\hline$\epsilon_{z}$ & $\pi \mathrm{mm} \mathrm{mrad}$ & 0.18 & 0.74 & 0.21 \\
\hline$\epsilon_{t}$ & $\pi \mathrm{mm} \mathrm{mrad}$ & 0.258 & 0.27 & 0.255 \\
\hline Energy & $\mathrm{MeV}$ & 3028.32 & 3027.5 & 3028.45 \\
\hline
\end{tabular}

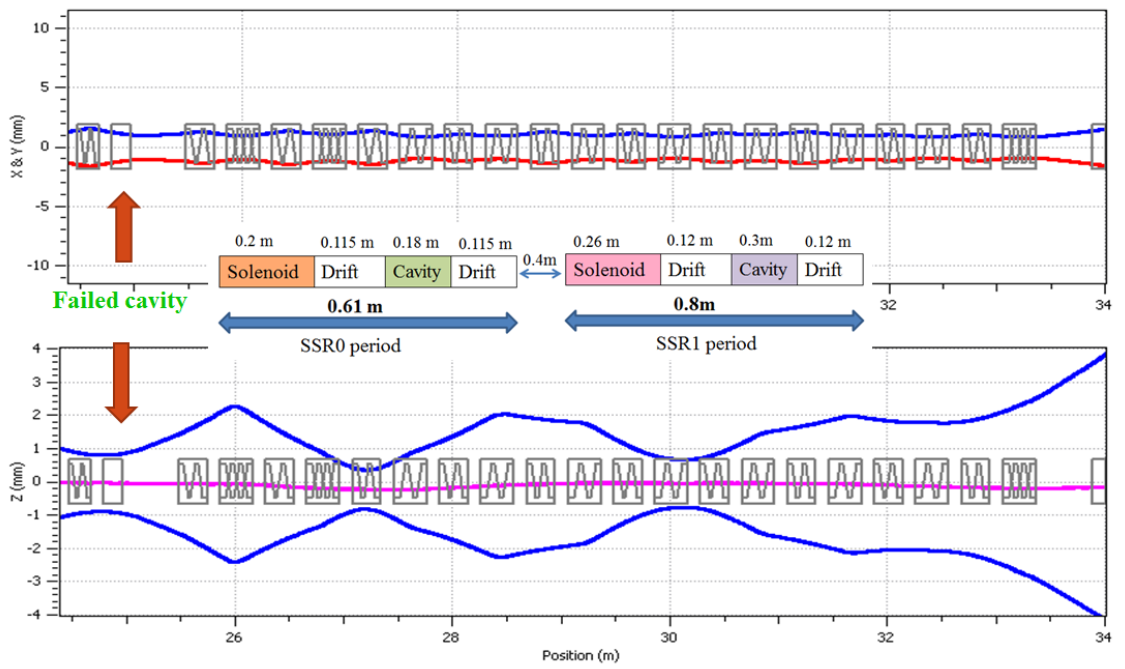

Figure 4.17: Beam transverse envelope (top) and longitudinal envelope (bottom) in presence of failure of last cavity in SSR0 section.

transverse dynamics remains nearly unaffected. Beam losses are observed at the same location as in earlier case and rms bunch length (longitudinal beam envelope) looks similar to Figure $4.12 \mathrm{~b}$,

Local compensation scheme is applied and two periods of SSR0 sections before and two periods of SSR1 after the failed cavity are used to retune the lattice. Beam energy is restored after the end of two periods of SSR1 section and $100 \%$ beam transmission trough the linac is obtained. Beam quality parameters, before and after compensation, are compared. Figure 4.18a shows longitudinal beam emittance before (green) and after compensation (red). Longitudinal beam emittance after applying local compensation is plotted on secondary y axis. Beam emittnace growth is recovered within $6 \%$ growth 


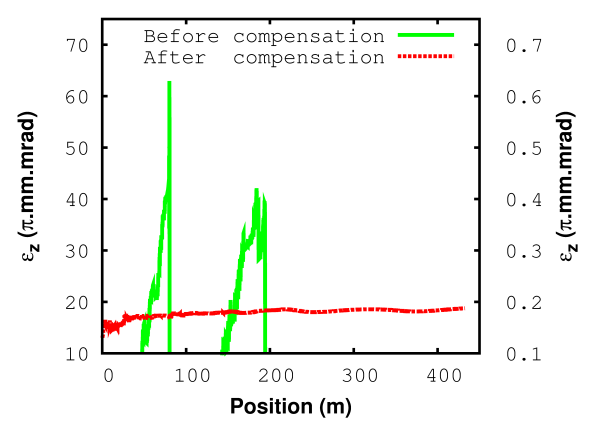

(a)

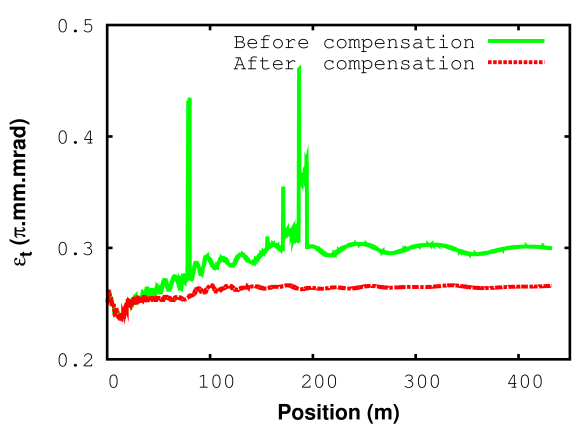

(b)

Figure 4.18: Beam emittance before and after applying correction scheme in (a) longitudinal plane (after compensation is plotted on right y-axis) and (b) transverse plane.

Table 4.7: Beam parameters at the end of linac.

\begin{tabular}{|c|c|c|c|c|}
\hline \multirow[t]{2}{*}{ Parameters } & \multirow[t]{2}{*}{ Unit } & \multirow[t]{2}{*}{ Nominal lattice } & \multicolumn{2}{|c|}{$\begin{array}{l}\text { Lattice with failure } \\
\text { of last SSR0 cavity }\end{array}$} \\
\hline & & & $\begin{array}{c}\text { Before } \\
\text { compensation }\end{array}$ & $\begin{array}{c}\text { After } \\
\text { compensation }\end{array}$ \\
\hline$\epsilon_{z}$ & $\pi \mathrm{mm} \mathrm{mrad}$ & 0.18 & 0.48 & 0.19 \\
\hline$\epsilon_{t}$ & $\pi \mathrm{mm} \mathrm{mrad}$ & 0.258 & 0.30 & 0.265 \\
\hline Energy & $\mathrm{MeV}$ & 3028.32 & 3025.143 & 3028.44 \\
\hline
\end{tabular}

with respect to nominal beam emittance. Figure $4.18 \mathrm{~b}$ shows transverse emittance before compensation (green) and after compensation (red). Emittance growth and beam energy at the end of linac are summarized in Table 4.7. It can be seen that emittance and energy are restored to large extent after applying local compensation.

\subsubsection{Study and compensation of failure of last cavity in SSR2 section}

In SC CW linac frequency jump is made at the end of SSR2 section. Frequency jump from $325 \mathrm{MHz}$ to $650 \mathrm{MHz}$ is very sensitive region in lattice. It is designed very carefully to minimize possible shrinkage in phase space available for particles in bunch. Thus, failure of outermost elements in both sides of transition disturb necessary matching condition which can lead to beam losses. Study is performed to understand the effects 
of failure of last cavity in SSR2 section. It is found that failure of last cavity in SSR2 section results in reduction of beam energy about $8 \mathrm{MeV}$ at the end of linac. Emittance growth of less than $10 \%$ is observed. However, no beam particles are lost and $100 \%$ beam transmission is observed. It can be concluded that beam profile is not as sensitive to failure of RF cavity as it was in low energy section (SSR0) of the linac. It is due to higher beam energy which makes it more rigid against surface charge forces. The beam energy is about $160 \mathrm{MeV}$ at the end of SSR2 section and it has sufficiently higher velocity relative to beam velocity at SSR0 section. Thus, magnitude of surface charge force and hence beam size is much lower which reduces the amplitude of beam mismatch. However, local compensation is applied to restore final beam energy at the end of the linac. Neighbouring elements in both sides of transition in the vicinity of failed RF cavity are retuned to restore the beam energy after few periods in $\beta_{G}=0.61$ section. Beam energy and beam emittances after local compensation are summarized in Table 4.8 .

Table 4.8: Beam parameters at the end of linac.

\begin{tabular}{|c|c|c|c|c|}
\hline \multirow[t]{2}{*}{ Parameters } & \multirow[t]{2}{*}{ Unit } & \multirow[t]{2}{*}{ Nominal lattice } & \multicolumn{2}{|c|}{$\begin{array}{l}\text { Lattice with failure } \\
\text { of last SSR2 cavity }\end{array}$} \\
\hline & & & $\begin{array}{c}\text { Before } \\
\text { compensation }\end{array}$ & $\begin{array}{c}\text { After } \\
\text { compensation }\end{array}$ \\
\hline$\epsilon_{z}$ & $\pi \mathrm{mm} \mathrm{mrad}$ & 0.18 & 0.194 & 0.19 \\
\hline$\epsilon_{t}$ & $\pi \mathrm{mm} \operatorname{mrad}$ & 0.258 & 0.267 & 0.255 \\
\hline Energy & $\mathrm{MeV}$ & 3028.32 & 3020.0 & 3029.57 \\
\hline
\end{tabular}

\subsubsection{Failure of magnetic element}

Study has been performed to analyze the effects of failure of magnetic elements (solenoid) in lattice. Impact of the failure of a cavity or magnetic element also depends on their location in a lattice. The situation is more critical when ratio between physical aperture (beam pipe aperture) and beam size is small. It is common situation at low energy part of the linac where space charge effects are considerable and beam has large transverse size. Scenario of failure of magnetic elements are analyzed for most critical cases in lattice i.e., failure of first solenoid in SSR0 section and failure of solenoid at the first transition between SSR0 and SSR1 section (first solenoid in SSR1 section). 


\subsubsection{Study and compensation of failure of first solenoid in SSR0 section}

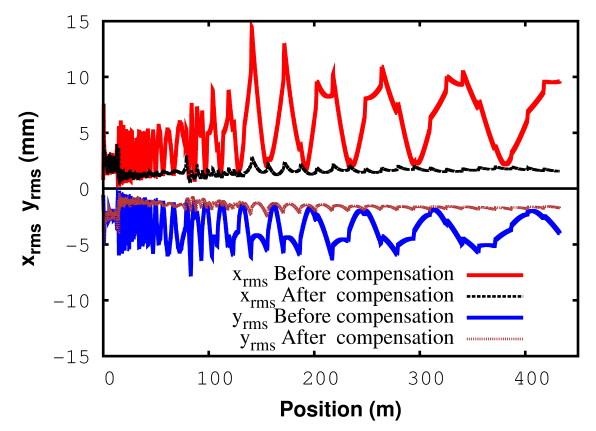

(a)

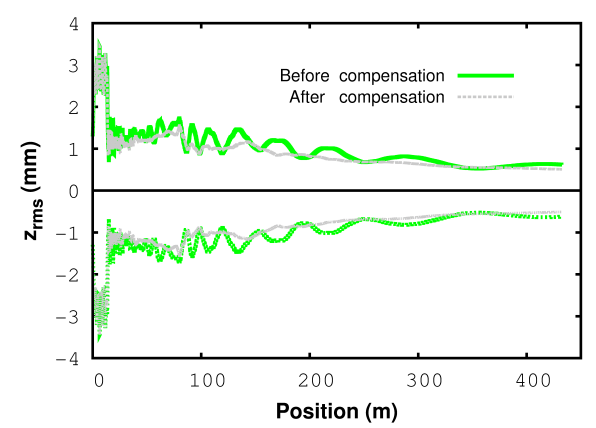

(b)

Figure 4.19: Beam envelopes before and after local compensation in (a) transverse plane and (b) longitudinal plane along the linac in presence of failed solenoid in SSR0 section.

Failure of first solenoid in SSR0 section is considered as critical as failure of first RF cavity in this section. Oscillation of beam envelope with large amplitude in transverse plane can be noticed in Figure 4.19a. Unlike the case of cavity failure, where transverse dynamics remains insensitive to any changes in longitudinal dynamics, disturbance in transverse dynamics due to failure of solenoid also couples with longitudinal dynamics which can be seen in Figure 4.19b Failure of solenoid results in huge beam losses. Local compensation scheme is applied using same approach what we used for failure of RF cavity. Neighbouring elements (solenoids and cavities) in the vicinity of failed solenoid are retuned in order to achieve smooth beam propagation and minimum beam losses. Transverse and longitudinal beam envelopes after local compensation can also be seen in Figures $4.19 \mathrm{a}$ and $4.19 \mathrm{~b}$ respectively.

No beam losses are observed after local compensation and beam emittances are also restored. Figure 4.20a shows beam transverse emittance before (red) and after (blue) applying local compensation. Comparison of longitudinal emittance growth before and after applying local scheme can be seen in Figure 4.20b, Beam emittances and beam energy at the end of linac are summarized in Table 4.9 . 


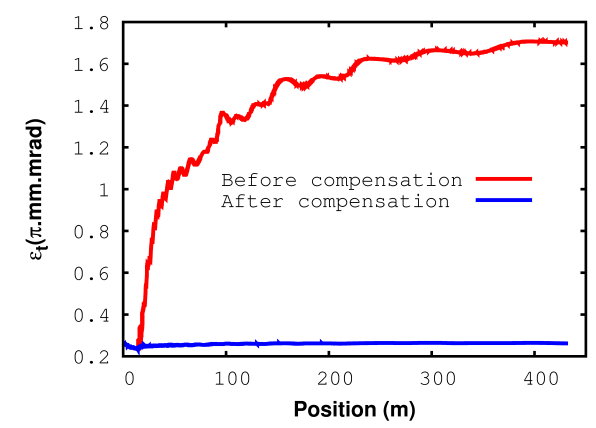

(a)

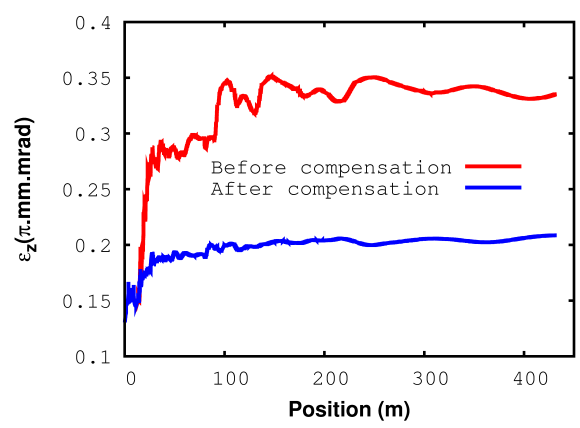

(b)

Figure 4.20: (a) Transverse and (b) longitudinal normalized r.m.s. emittance along the linac with and without compensation scheme in presence of failure of first solenoid in SSR0 section .

Table 4.9: Beam parameters at the end of linac.

\begin{tabular}{|c|c|c|c|c|}
\hline \multirow[t]{2}{*}{ Parameters } & \multirow[t]{2}{*}{ Unit } & \multirow[t]{2}{*}{ Nominal lattice } & \multicolumn{2}{|c|}{$\begin{array}{l}\text { Lattice with failure } \\
\text { of first SSR0 solenoid }\end{array}$} \\
\hline & & & $\begin{array}{c}\text { Before } \\
\text { compensation }\end{array}$ & $\begin{array}{c}\text { After } \\
\text { compensation }\end{array}$ \\
\hline$\epsilon_{z}$ & $\pi \mathrm{mm} \mathrm{mrad}$ & 0.18 & 0.34 & 0.208 \\
\hline$\epsilon_{t}$ & $\pi \mathrm{mm} \mathrm{mrad}$ & 0.258 & 1.7 & 0.262 \\
\hline Energy & $\mathrm{MeV}$ & 3028.32 & 3028.31 & 3028.44 \\
\hline
\end{tabular}

\subsubsection{Study and compensation of failure of first solenoid in SSR1 section}

As discussed earlier that outermost elements at the ends of both cryomodules are used to provide matching for smooth beam transition from one section to another. Thus, failure of first solenoid in SSR1 section is also considered as one of critical cases as beam is still non relativistic and surface charge effects are significant relative to high energy section. Thus, failure of first solenoid in SSR1 section results in large beam transverse size. It also causes mismatch of beam in transverse plane. Beam envelopes show similar behavior as shown in last case (Figure 4.19). Local compensation is applied to restore the nominal performance of the linac. Figure 4.21a shows comparison between transverse emittance before local compensation (red) and transverse emittance after 


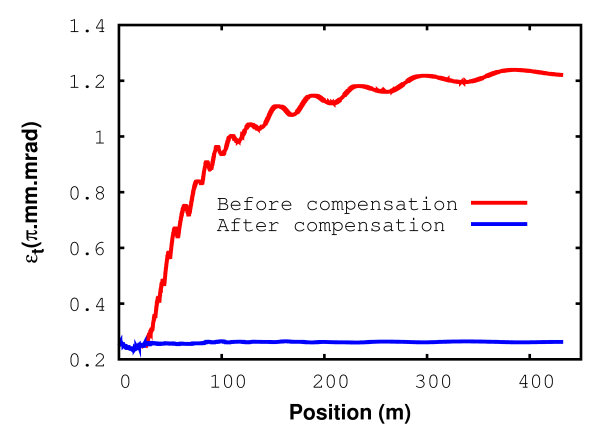

(a)

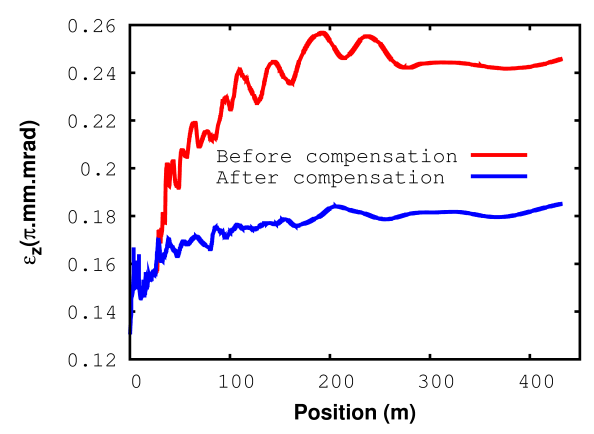

(b)

Figure 4.21: (a) Transverse and (b) longitudinal normalized r.m.s. emittance along the linac with and without compensation scheme in presence of failure of first solenoid in SSR1 section.

(blue) local compensation. Longitudinal emittnace is also recovered after applying local compensation (Figure 4.21b). Beam emittances with beam energy at the end of linac are summarized in Table 4.10.

Table 4.10: Beam parameters at the end of linac.

\begin{tabular}{|c|c|c|c|c|}
\hline \multirow[t]{2}{*}{ Parameters } & \multirow[t]{2}{*}{ Unit } & \multirow[t]{2}{*}{ Nominal lattice } & \multicolumn{2}{|c|}{$\begin{array}{l}\text { Lattice with failure } \\
\text { of first SSR1 solenoid }\end{array}$} \\
\hline & & & $\begin{array}{c}\text { Before } \\
\text { compensation }\end{array}$ & $\begin{array}{c}\text { After } \\
\text { compensation }\end{array}$ \\
\hline$\epsilon_{z}$ & $\pi \mathrm{mm} \mathrm{mrad}$ & 0.18 & 0.25 & 0.185 \\
\hline$\epsilon_{t}$ & $\pi \mathrm{mm} \mathrm{mrad}$ & 0.258 & 1.23 & 0.263 \\
\hline Energy & $\mathrm{MeV}$ & 3028.32 & 3028.31 & 3028.43 \\
\hline
\end{tabular}

It can be concluded from analysis of various scenario of failure of beam line elements at the critical locations in linac that the lattice design is robust enough to operate linac even with failed elements after applying local compensation scheme. It is found that failure of beam line element at low energy is very critical and may result in beam losses in absence of its compensation. The local compensation allows restoring of beam energy and beam emittance. It is possible to achieve $100 \%$ beam transmission after local compensation of failed elements. 


\section{5}

\section{Cavity fabrication and characterization}

This chapter describes fabrication steps for cavity design. A cumulative experience of SCRF community is applied to develop technique that describes the manufacturing steps of cavities which can exceed gradient of $30 \mathrm{MV} / \mathrm{m}$. This chapter introduces manufacturing processes within the framework of $1.3 \mathrm{GHz}, 9$-cell, $\beta_{G}=1$ cavities. Cavity characterization and quality assessment at different stages of fabrication are also discussed. It should be noted that same procedure will be considered for fabrication of the $\beta_{G}=0.61$ and $\beta_{G}=0.90$ cavities for the $\mathrm{CW}$ linac of the Project-X facility.

\subsection{Cavity fabrication}

SCRF cavity is a complex and expensive device. Fabrication of cavity involves lot of engineering efforts. Different methods have been employed for SCRF cavity fabrication such as machining from solid piece, forming half-cell from niobium $(\mathrm{Nb})$ sheet and depositing $\mathrm{Nb}$ film on preformed cavity substrate. The mass production of cavities especially at low frequencies using method of machining from solid piece of $\mathrm{Nb}$ is not possible for economical reasons while accelerating gradient is limited in $\mathrm{Nb}$ sputtered cavities. Thus, several sheet metal forming techniques such as spinning, hydroforming and deep drawing have been developed. Deep drawing method is most established technique for cavity fabrication and widely used in industries for the fabrication of elliptical shape cavity . A Cavity journey from its fabrication to its installation in 


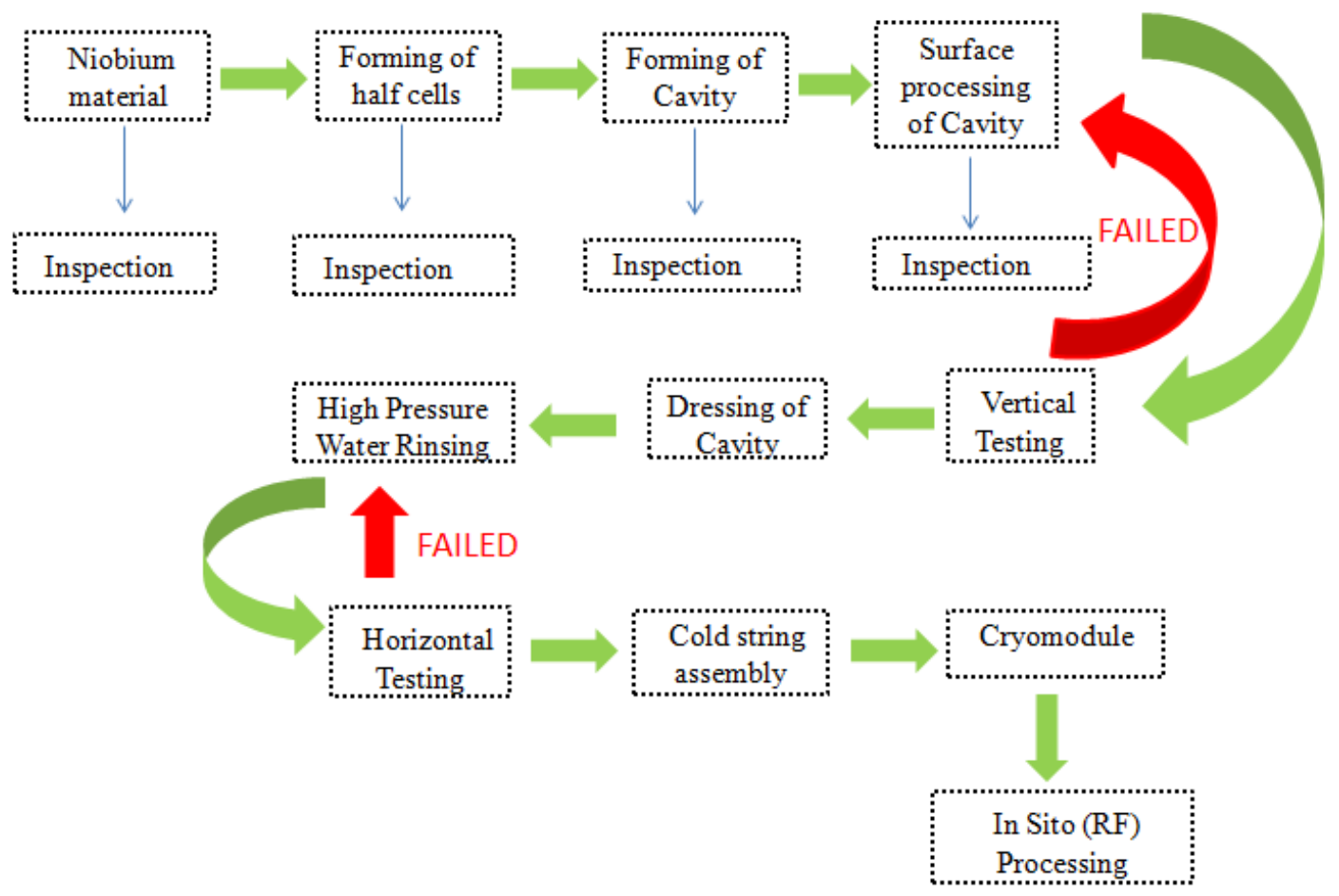

Figure 5.1: Schematic of cavity fabrication process.

accelerator is summarized in Figure 5.1 .

\subsubsection{Niobium sheet}

Accelerating gradient in SCRF cavities are limited by material properties. Thus, $\mathrm{Nb}$ sheet are inspected for surface quality such as absence of scratches, RRR value, sheet flatness and uniform grain size. Eddy current scanner is used to inspect these Nb sheets. The eddy current method is based on the principle of generating circular electrical currents in a material under test, It can be achieved by the use of a driving alternating magnetic field (primary field). The induced eddy current within the inspected material will itself produce a magnetic field (secondary field) in the direction opposite to the primary field (Lenz's law). Magnitude of induced current will be different for different materials and for damaged surfaces. Thus, variation in induced current reflects the volume of material inclusion (impurities) and the depth of defect. 


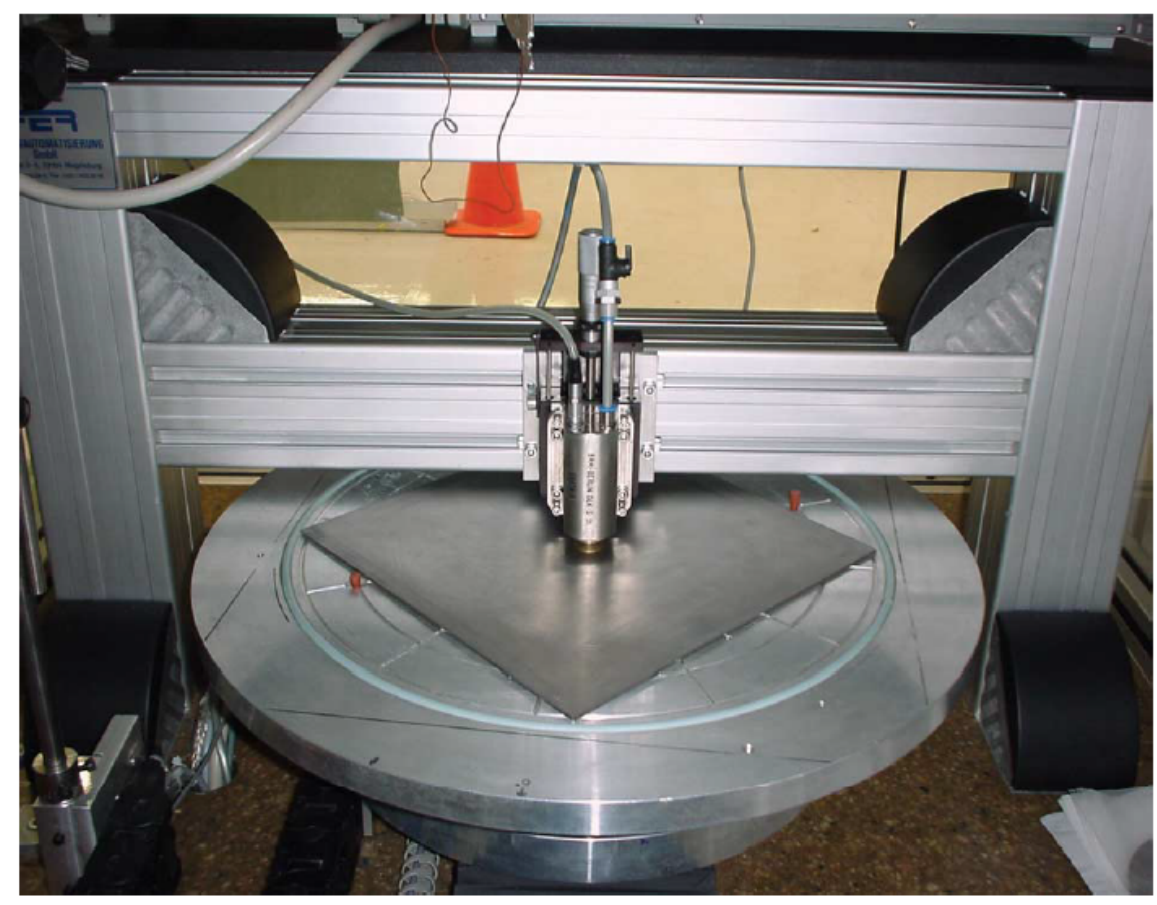

Figure 5.2: Eddy current scanner setup at Fermilab.

Setup for eddy current scanner at Fermilab is shown in Figure 5.2. In this particular machine, the sample is fixed to a rotating table, which is attached to a linear slider, while the sensing probe is kept fixed. Variation in eddy currents will result in generation of magnetic field in a double coil sensing probe. Thus, it detects inclusions and defects embedded under the surface of the material. This setup is specifically designed to scan disks. At a given relative radial position of the head, the sample rotates several times allowing for data acquisition, then the relative position of the head is changed by sliding the table and the data acquisition is repeated over a different circumference. The sensing probe generates two signals at different frequencies for simultaneous investigation over two depths. The signals are then digitalized and transferred to a PC where the data is stored and represented in the form of pictures as shown in Figure 5.3 .

\subsubsection{Forming half cells: Deep drawing method}

After inspection, Nb sheets are used for forming half cells. "Blank" which is usually $\mathrm{Nb}$ disk made from $\mathrm{Nb}$ sheet is pressed into the shape using a set of dies. Figure 5.4 


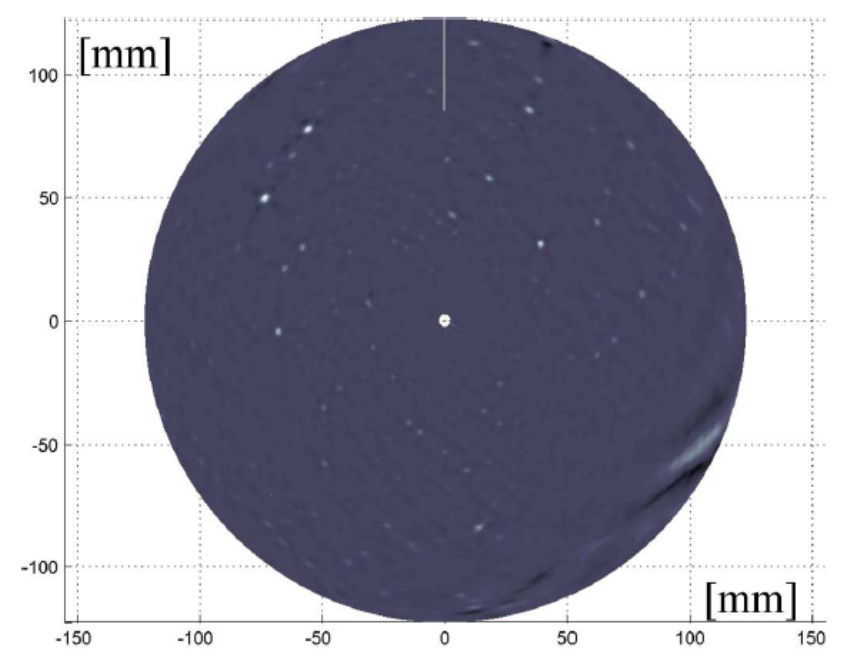

Figure 5.3: Scanned result of $\mathrm{Nb}$ sheet. The white spots correspond to pits on the surface of the material.

shows schematic of dies. Dies are usually made from a high yield strength aluminium alloy.
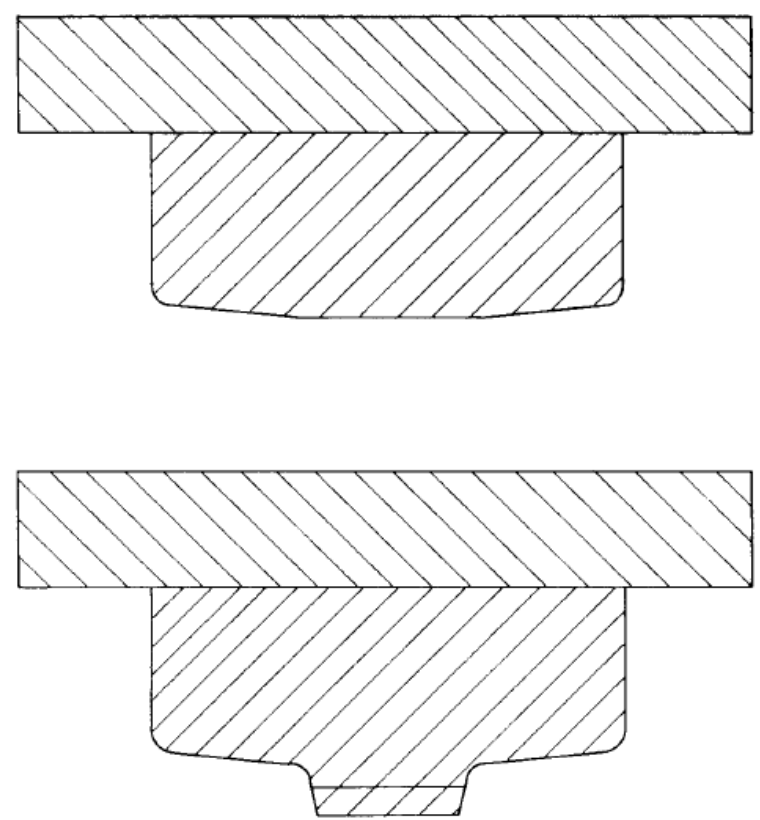

Figure 5.4: Deep drawing tools for half cell formation- Male die (top) and female die with holding plate (bottom). 


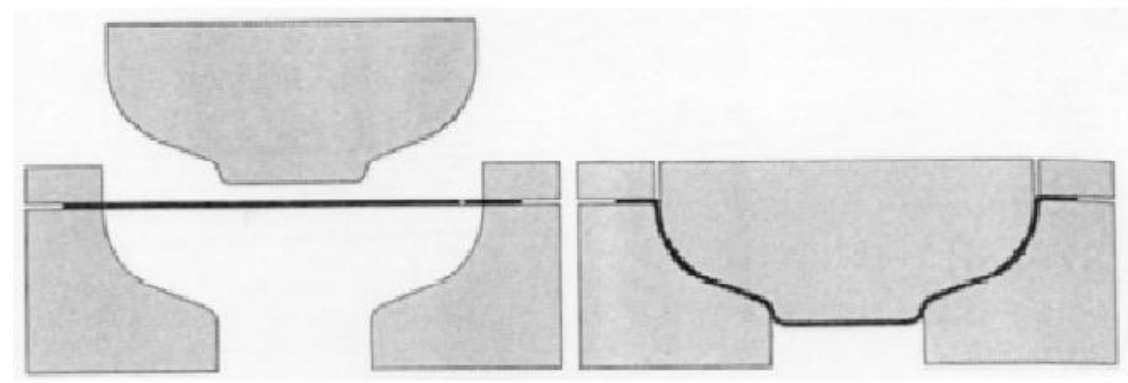

Figure 5.5: Deep drawing method for half cell forming.

Typical half cell formation is shown in Figure 5.5. Nb disk is bolted across the female die with hold down plate. With appropriate torque on the bolts, the outer edge of $\mathrm{Nb}$ is constrained without tearing at clamped edge. Clean motor oil is painted onto the $\mathrm{Nb}$ for lubrication. The male die is placed in position and the assembly is squeezed in a hydraulic press. For the $1.3 \mathrm{GHz}$ half cell, 100 tons of force is applied. To get the curvature required at the iris, the nose of cup is coined with a coining ring and the male die. Figure 5.6a shows forming tools developed at Raja Ramanna center for advance technology (RRCAT) in India. A simulated sketch (using ANSYS [73]) for half cell cup is shown in Figure $5.6 \mathrm{~b}$,

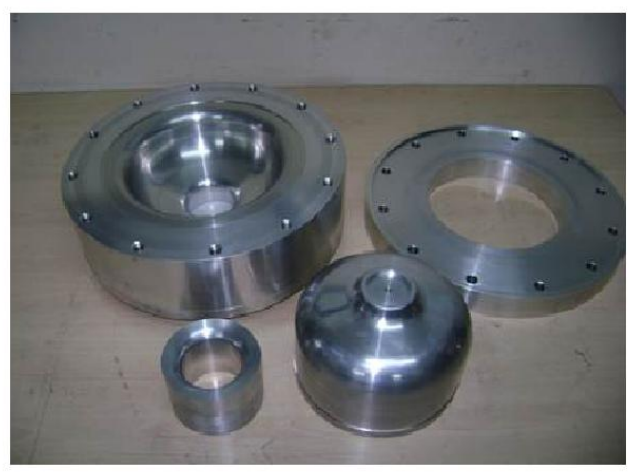

(a)

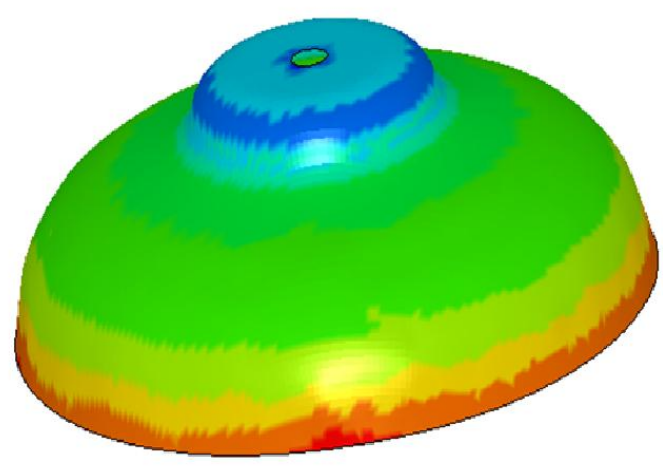

(b)

Figure 5.6: (a) Half cell forming tools developed at RRCAT and (b) a sketch of half cell.

To achieve tight tolerances and good reproducibility one has to deal with following difficulties: 


\section{CAVITY FABRICATION AND CHARACTERIZATION}

- Thickness tolerances of material.

- Removing the parts from the die is sometime difficult and may result in dimensional changes.

- Elastic property of $\mathrm{Nb}$ may vary from one sheet to another.

After forming cups (half cell), trimming is required to achieve final size for electron beam welding (EBW). Trimming at iris and equator includes welding shrinkage too. The accuracy of the shape is controlled by sandwiching the half-cell between two metal plates and measuring the resonance frequency. Soaking the cups in dilute hot sulphuric acid for a few hours is a simple method to remove any embedded iron particles (e.g., from the rolling mill). The cups are checked by a rust test for any remaining iron inclusions by soaking in water for 12 hours. Half cells are given a light etch $(20 \mu \mathrm{m}$ material removal) before EBW. After chemical treatment, it is rinsed throughly and dried in a clean room then kept clean in sealed nylon bags before welding.

\subsubsection{Electron beam welding (EBW)}

Two half cells are welded from equator and beam pipe is welded at iris to form a single cell cavity while two half cells are welded at iris to form dumb-bells. Dumb-bells are further welded with each other at equator to fabricate multi-cell cavity. All the parts are welded together in a vacuum chamber to avoid RRR degradation. The pressure in a chamber should be less than $2 \times 10^{-5}$ torr.

The welded parameters are chosen to achieve full penetration "butt" welds with smooth under bead by using a defocused electron beam. A focus beam can produce weld spatter, resulting in weakly attached $\mathrm{Nb}$ beads. One technique to produce a well defined and reproducibly defocused beam [24] is shown in Figure 5.7. Beam is deflected in the shape of a rhombus with the beam deflection yoke. Resulting defocus beam causes reduction in energy density which produce under-bead. Careful control of material thickness in order to achieve full penetration with no blow holes is required.

\subsubsection{Inspections and qualification tests:}

After fabrication, cavity is passed through lot of qualification tests and inspections such as leak test, optical inspection, RF measurements etc. 


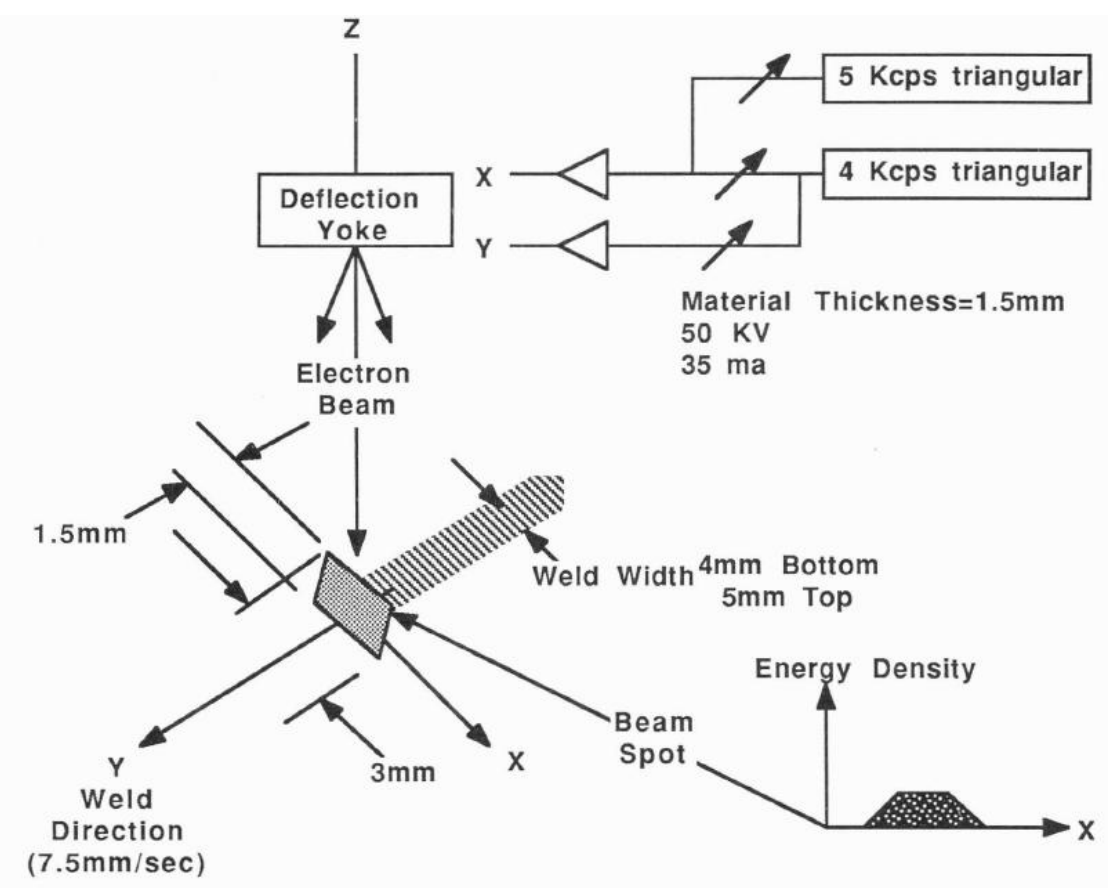

Figure 5.7: Production of defocused electron beam for EB welding [24].

\subsubsection{Vacuum leak test}

Cavity is tested for vacuum leak at room temperature and cold temperature. Inability to produce and maintain low pressure in cavity will show presence of leak. It might happen due to not fully penetrated weld seam. In some cases material removal after chemical treatment will also result in cavity leak. Table 5.1 shows vacuum leak test performed for one of the cavity (TE1CAT003) at RRCAT.

Table 5.1: Vacuum leak test for TE1CAT003 cavity after EB welding.

\begin{tabular}{cccc}
\hline Temperature & $\begin{array}{c}\text { Allowable } \\
\text { leak rate }\end{array}$ & $\begin{array}{c}\text { Measured } \\
\text { leak rate }\end{array}$ & Acceptance \\
\hline $300 \mathrm{~K}$ & $\leq 10^{-09} \mathrm{mbar} / \mathrm{sec}$ & $\leq 10^{-12} \mathrm{mbar} / \mathrm{sec}$ & Yes \\
$77 \mathrm{~K}$ & $\leq 10^{-09} \mathrm{mbar} / \mathrm{sec}$ & $\leq 10^{-12} \mathrm{mbar} / \mathrm{sec}$ & Yes \\
\hline
\end{tabular}




\section{CAVITY FABRICATION AND CHARACTERIZATION}

\subsubsection{Optical inspection}

Optical inspection of fabricated cavity is performed using high resolution camera system developed at KEK and Kyoto university. The high resolution pictures obtained with the system allows locating and studying defects on the inner cavity surface in a way that was not possible before without cutting samples from the cavity.

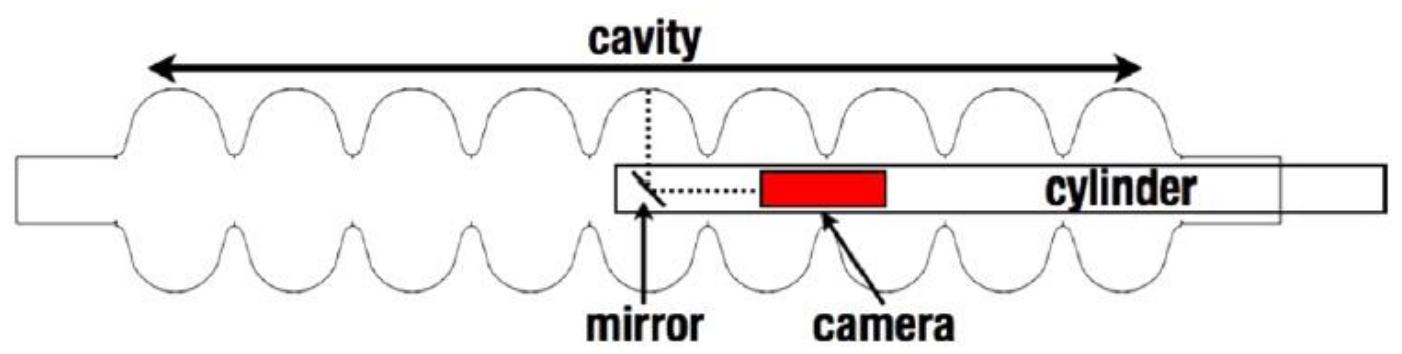

Figure 5.8: Schematic diagram of cavity inspection system. The cavity swallows the camera cylinder by moving longitudinally. The cylinder does not move. The inner surface reflected in an imaging mirror is observed. The mirror is located in front of the camera.

Figure 5.8 shows sketch of optical system. Camera is mounted in a cylindrical tube and its outer surface is covered with electroluminescence (EL) sheets which acts as a thin light source. The advantage of placing camera in a cylinder is that it eliminates the possibility of contact between cavity surface and camera. Thus, it provides nondestructive observation and keeps the surface clean and untouched. The mirror is placed at the head of cylinder and it reflects the inner surface of the cavity. The imaging mirror can be tilted by a pulse motor (PM) to show a surface other than the cavity equator. The focus is adjusted by motorized positioning of the camera that keeps the optical length (working distance) between the object and the camera lens constant. A cavity can be slid in the longitudinal direction and rotated around its axis, while the position of the camera cylinder is fixed so that the inner surface of every cell can be observed (Figure 5.9). The longitudinal position of the cavity is determined by the number of pulses fed to the PM, while the azimuthal coordinates are measured by the rotary encoder installed at the edge of a cavity flange. Both the longitudinal and azimuthal positioning resolutions are less than $5 \mu \mathrm{m}$, which should be enough for our purpose. The step resolution of the PM can be increased, using a special function of pulse motor 
controllers. Although the camera cylinder is fixed on the table, the movement of the cavity during its positioning shakes the cylinder. This disturbs the observation during the swing because of the very narrow depth of field of camera. A damper installed at the end of the cylinder helps to damp the swing quickly.

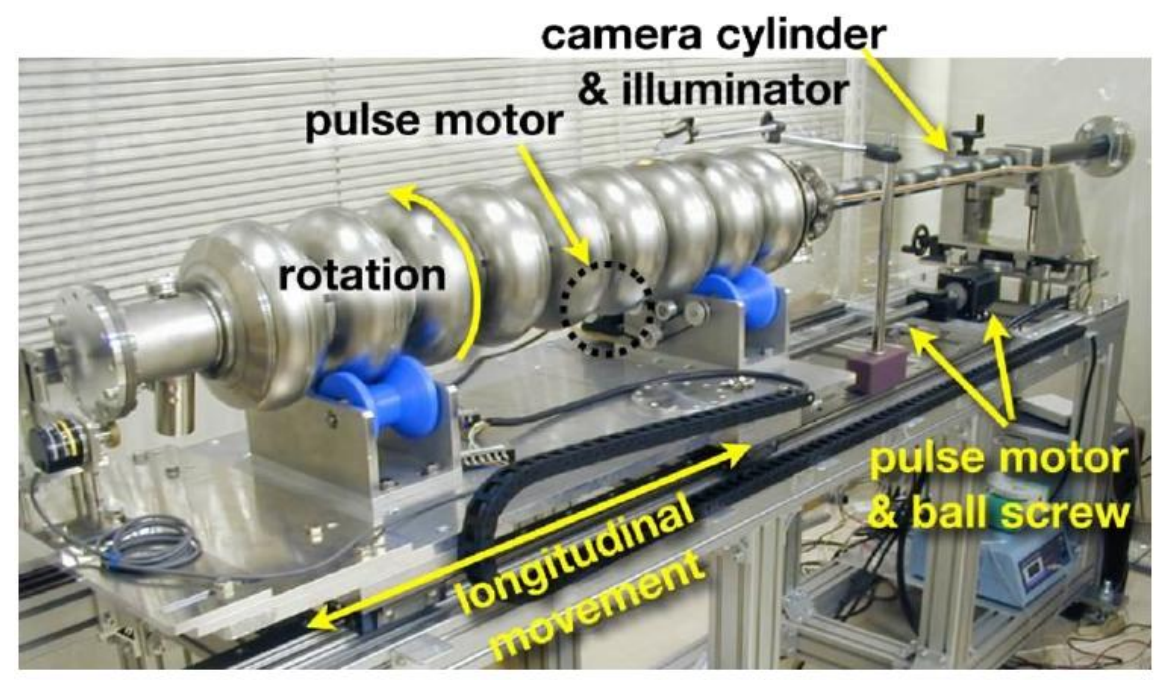

Figure 5.9: Overview of optical inspection system at Fermilab.

Optical inspection of TE1CAT003 cavity after EB welding is performed at Fermilab and Figures $5.10 \mathrm{a}$ and $5.10 \mathrm{~b}$ show images of inner surface of cavity around the equator region.

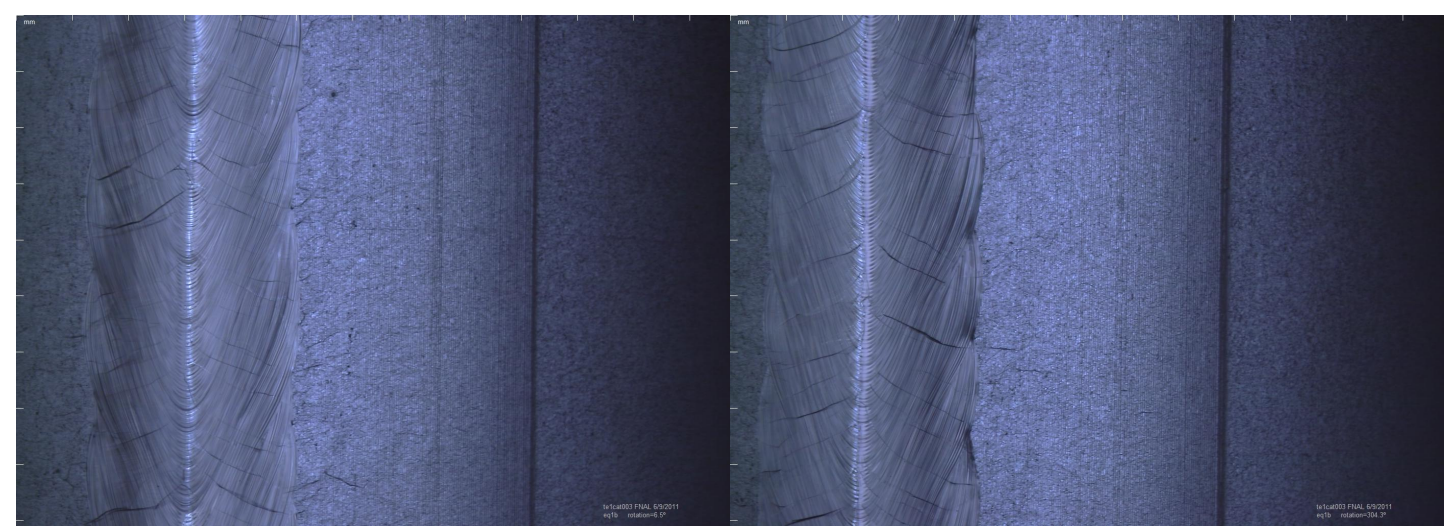

(a)

(b)

Figure 5.10: Images of inner surface of TE1CAT003 cavity. 


\section{CAVITY FABRICATION AND CHARACTERIZATION}

\subsection{Cavity surface processing}

It is well known that RF superconductivity is taking place in a thin surface layer of only few of nano meter in microscopic scale. Thus, an excellent quality of inner surface is required to obtain high gradient and high quality factor in SCRF cavities. Smooth and clean surface on a microscopic level results in smaller surface resistance than for a rough surface due to shorter current path (mean free path of electrons) and therefore, less power dissipation takes place in cavity. It has also been understood that rough RF surfaces lead both to electric field enhancement causing field emission and to magnetic field enhancements initiating thermal breakdown.

Beside the defects and impurities of starting material, fabrication of cavity also introduces damaged layer due to mechanical deformation of lattice during forming, dirt and other defects like scratches on inner surfaces, bump etc. Thus, to achieve the inner surface of cavity as close as possible to ideal for optimal RF performance, cavity is passed through sequence of surface treatments in order to remove defects from production processes. Initial step is to remove material (etching) from the inner surface. It is named as surface preparation of cavity. Figure 5.11a and 5.11b show typical nature

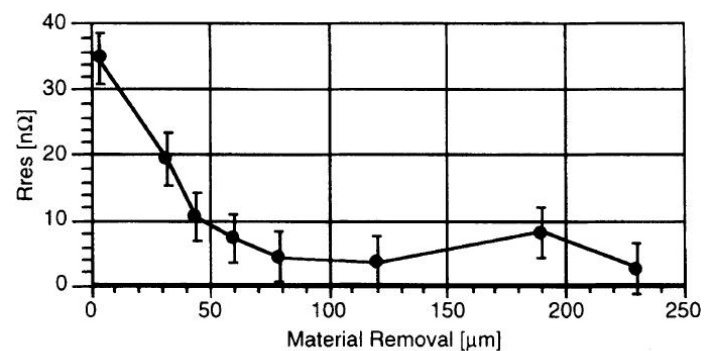

(a)

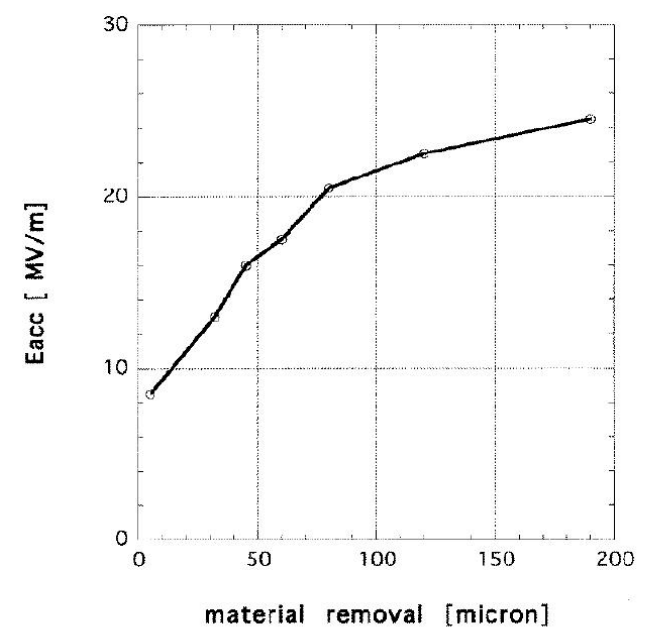

(b)

Figure 5.11: Effect of successive material removal -(a) surface resistance decreases and (b) accelerating gradient increases as a consequence [74.

of surface resistance and accelerating gradient after successive removal of damaged $\mathrm{Nb}$ 
layer from inner surface of cavity. Surface resistance decreases which results in achieving higher accelerating gradient in cavity. Buffer chemical polishing (BCP) and Electro polishing (EP) are two common processes which are used for material removal for RF cavities.

Buffer chemical polishing: BCP has been accepted worldwide as a technique for removing damaged and contaminated inner surface layers of cavity. This method uses a mixture of three acids i.e., hydroflouric acid (HF) with concentration of $40 \%$, Nitric acid $\left(\mathrm{HNO}_{3}\right)$ with concentration of $65 \%$ and Orthoposphoric acid $\left(\mathrm{H}_{3} \mathrm{PO}_{4}\right)$ with concentration of $85 \%$. Cavity is filled by this mixture. HF reacts with the $\mathrm{Nb}_{2} \mathrm{O}_{5}$ and dissolved it. As $\mathrm{HF}$ dissolves pure $\mathrm{Nb}$ very slowly so a strong oxidising agent $\mathrm{HNO}_{3}$ is required which re-oxidises the $\mathrm{Nb}$ and form $\mathrm{Nb}_{2} \mathrm{O}_{5}$. Nitric acid reacts with niobium to form niobium pentoxide $\left(\mathrm{Nb}_{2} \mathrm{O}_{5}\right)$. HF reacts with $\mathrm{Nb}_{2} \mathrm{O}_{5}$ to form niobium fluoride $\left(\mathrm{NbF}_{5}\right)$. This compound is hydrosoluable and goes into solution thus resulting in a polished $\mathrm{Nb}$ surface. $\mathrm{H}_{3} \mathrm{PO}_{4}$ acid serves as the buffer to maintain the reaction rate. The etching process is exothermic and rate of $\mathrm{Nb}$ removal depends on temperature and amount of $\mathrm{Nb}$ in acid mixture. Acceptable etching rate is achieved if the concentration of $\mathrm{Nb}$ does not exceed more than $1.5 \mathrm{~g} / \mathrm{l}$ in solution. It has been found that $\mathrm{BCP}$ results in hydrogen contamination at the surface which leads to degradation in quality factor of cavity. This problem can be overcome by using appropriate proportion of acid and buffer and by keeping the temperature below $15^{\circ} \mathrm{C}$. The proportion of $\mathrm{HNO}_{3}$, $\mathrm{HF}, \mathrm{H}_{3} P_{4}$ is 1:1:2 in mixture by volume. It is found that removal of $\mathrm{Nb}$ from cavity surface by BCP process is not uniform. Ratio of material removal from iris to equator is 2:1. Experience with $1.3 \mathrm{GHz}, \beta_{G}=1 \mathrm{SCRF}$ ILC cavities show that $30 \mathrm{MV} / \mathrm{m}$ seems to be maximum gradient for those cavities which undergo BCP.

Electro polishing (EP): It is prominent method to remove damaged layers from inner surface of cavity. Sharp edges and variations are smoothed out and a very glossy surface can be obtained. The major advantage of this method is a much improved control of the chemical reaction, as a further control parameter namely the electric voltage is introduced. During the EP process the Nb cavity is half filled with mixture of acid and rotated. Schematic of EP for half cell and single cell cavity is shown in Figure 5.12. Figure 5.13 shows EP system for ILC cavity at Argonne National Laboratory (ANL). In EP process cavity works as the anode and the cathode is a tube made from pure aluminium, which is placed some millimeters off the axis of the beam tube. By 


\section{CAVITY FABRICATION AND CHARACTERIZATION}
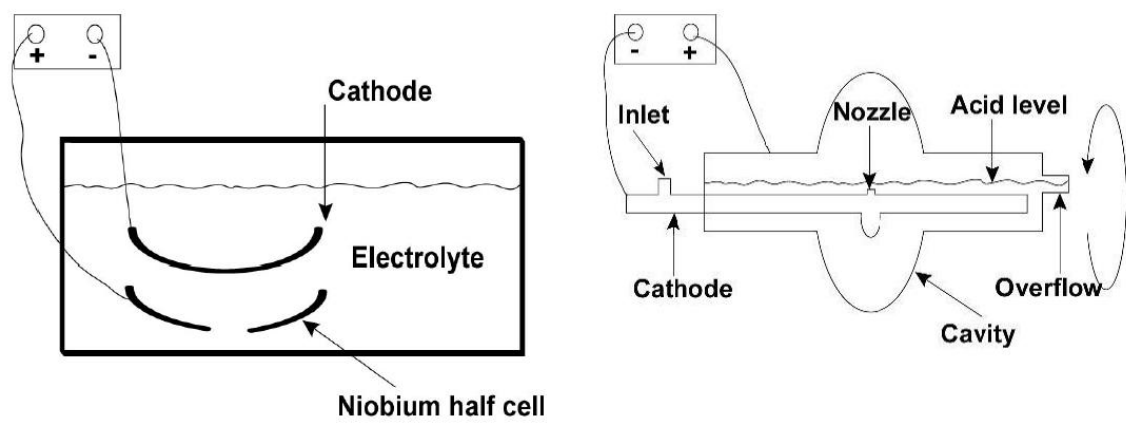

Figure 5.12: Schematic of EP system for half cell (left) and single cell (right).

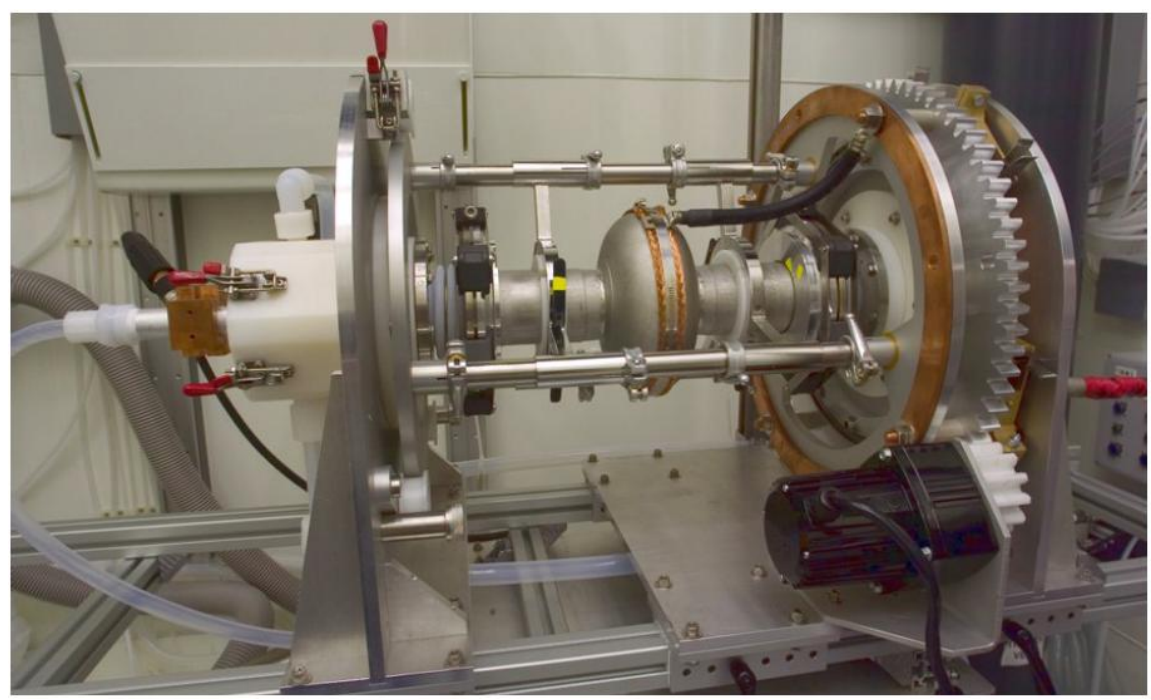

Figure 5.13: EP system for $1.3 \mathrm{GHz}$ ILC cavity at ANL.

applying a voltage of $10-20 \mathrm{~V}$ the polishing process is activated. The current oxidizes the $\mathrm{Nb}$ surface first at the peaks because of the voltage enhancement. Hydrofluoric acid solutes these oxides. This process has a smoothening effect to the Nb surface. The electro chemical dissolution of the $\mathrm{Nb}$ anodes surface take place in three steps ([75]):

$$
\begin{array}{r}
2 \mathrm{Nb}+5 \mathrm{SO}_{4}^{2-}+5 \mathrm{H}_{2} \mathrm{O} \rightarrow \mathrm{Nb}_{2} \mathrm{O}_{5}+10 \mathrm{H}^{+}+5 \mathrm{SO}_{4}^{2-}+10 e^{-} \\
\mathrm{Nb}_{2} \mathrm{O}_{5}+6 \mathrm{HF} \rightarrow \mathrm{H}_{2} \mathrm{NbOF}_{5}+\mathrm{NbO}_{2} \mathrm{~F} \bullet 0.5 \mathrm{H}_{2} \mathrm{O}+1.5 \mathrm{H}_{2} \mathrm{O} \\
\mathrm{NbO}_{2} \mathrm{~F} \bullet 0.5 \mathrm{H}_{2} \mathrm{O}+4 \mathrm{HF} \rightarrow \mathrm{H}_{2} \mathrm{NbOF}_{5}+1.5 \mathrm{H}_{2} \mathrm{O}
\end{array}
$$

At the aluminium cathode $\mathrm{H}_{2}$ will be formed and gassed out. In addition an undesired 
electrolysis of water, which is a constituent part of the acid mixture, is possible.

Comparison between EP and BCP: EP and BCP both are widely used worldwide. One of them is chosen to serve the purpose. A detailed comparison of EP and $\mathrm{BCP}$ on the cavity performance is presented in [76]. The key points are summarized here:

- EP has a little bit complicated procedure and needs annealing to avoid the hydrogen Q-disease. On the other hand, chemical polishing (CP) is easier and cheaper.

- EP provides mirror like smooth surface of cavity. Surface roughness of electro polished cavity is of the order of $1 \mu \mathrm{m}$ while it is $5 \mu \mathrm{m}$ for chemical polished cavity.

- Average maximum gradient achieved by cavities which undergoes BCP is about $25 \mathrm{MV} / \mathrm{m}$ while it is about $35 \mathrm{MV} / \mathrm{m}$ for cavities which are treated with EP.

- EP is slow as compared to BCP.

Barrel polishing (BP) or Tumbling: BP is usually applied to those cavities which consists of irregularities such as bump, scratches and sputter balls by EBW failure. $\mathrm{BP}$ followed by light $\mathrm{EP}$ or $\mathrm{BCP}$ is proved very effective and successful technique to deal with these defects. It is a kind of mechanical grinding in which plastic chips binding ceramic powder and liquid soap (collectively called media) are put in a cavity, then cavity is rotated horizontally. Chips size, rotation speed, number of chips and amount of soap are controllable parameters. BP is performed in sequences with different size of chips. It is very slow process and not the part of standard fabrication procedure.

Oxipolishing (OP): It is another important electrochemical process for the surface preparation of $\mathrm{Nb}$. The $\mathrm{Nb}$ surface is anodized using an electrolyte. Various electrolyte can be used, but the most common for $\mathrm{Nb}$ are diluted $\mathrm{NH}_{4}(\mathrm{OH})$, diluted $\mathrm{HNO}_{3}$ and diluted $\mathrm{H}_{2} \mathrm{SO}_{4}$. The thickness of the oxide layers produced can be adjusted with the applied voltage. For $50 \mathrm{~V}$ one gets a layer of $100 \mathrm{~nm}$. In a second step this layer is dissolved with HF. One can regard this as a step-by-step EP. The advantage of OP is that very tiny thicknesses can be removed. But this implies that for a removal of only $1 \mu \mathrm{m}$ already several steps are needed. It is important to note that contaminations like fluorine or sulphur from (electro-)chemical preparations can be removed very efficiently 


\section{CAVITY FABRICATION AND CHARACTERIZATION}

by oxipolishing. It may also be noted that anodization can also be used to form a protective layer of dielectric oxide on the $\mathrm{Nb}$ surface

After chemical treatment electrolyte is drained from cavity and it is rinsed with water to avoid chemical residuals. First, cavity is filled with de-ionized water and then drain it completely. This procedure is performed several times in sequence. This type of rinsing is then followed by extensive over flow rinsing while monitoring the $\mathrm{pH}$ or water resistivity. These procedures will adequately remove any excess acids trapped in the system as well as make the subsequent handling safe for personnel.

The electro polished or chemical polished surfaces are contaminated with reaction products such as lower oxides, sulphur and fluorine. Sulphur is able to settle and stick aggressively on the cavity surface and it is a well known source of field emission. Alcohols are good solvent of sulphur. Thus, cavities are rinsed with ethanol. It has been shown to substantially reduce field emission in 9-cell cavity tests.

High pressure water rinsing (HPWR): In order to reduce field emission in high gradient SCRF cavities, high pressure rinsing of ultra pure water has been adopted by numerous laboratories. It improves quality of the inner surface of cavity by removing contaminated particles and dirts. These contaminants may have been swimming in the acid bath or have been airborne and landed on the surface after the cavity was removed from acid. Figure 5.14 shows a schematic of typical HPWR system. It consists of a high pressure pump, a filter, a spray nozzle and a mechanical system, which allows the scanning of the interior surface with the high pressure water jet. Needle point jets of ultra pure water with pressure of the order of 1000psi are bombarded on cavity surface. The scanning system moves the cavity up and down while also rotating either it or jet along the axis of cavity so that complete inner surface can be covered. Both up-and-down speed are adjustable by means of motors with variable speed controls.

Heat treatment of cavity: During the process of EP, hydrogen is produced which can enter the $\mathrm{Nb}$ lattice very easily. It is well-known that the amount of interstitial hydrogen in very pure $\mathrm{Nb}$ must be kept below certain limits. If too much hydrogen is dissolved in the $\mathrm{Nb}$ a subtle effect called "Q-disease" can occur. The dissolved hydrogen forms $\mathrm{Nb}$ hydrides $(\mathrm{Nb}-\mathrm{H})$ at temperatures around $100 \mathrm{~K}$. These Nb-H compounds have very high microwave losses and raise the residual resistance by more than an order of magnitude. The concentration of hydrogen should be kept below the concentration levels of interstitials which can trap the hydrogen, so that no hydride formation can 


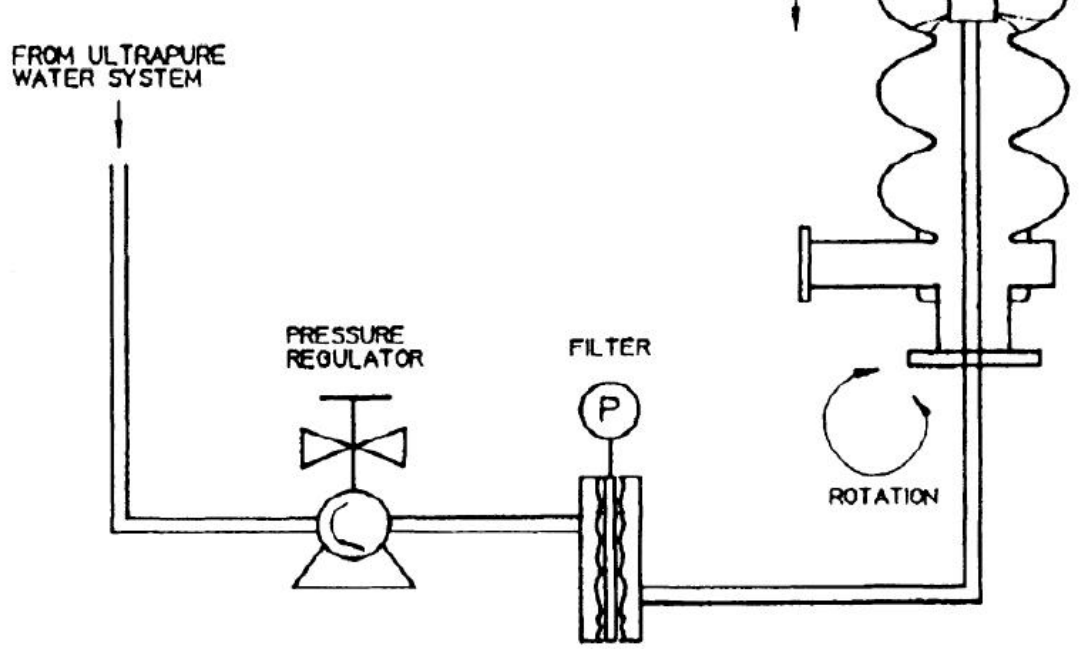

Figure 5.14: A schematic of high pressure water rinsing system.

take place. The cavities are baked at $800^{\circ} \mathrm{C}$ in high vacuum furnace for reducing hydrogen concentration. Recrystallization of $\mathrm{Nb}$ occurs at this temperature and lattice quality is improved afterwards. Mechanical stresses are released and lattice is less sensitive to further hydrogen contamination. There is also improvement in thermal conductivity at $2 \mathrm{~K}$ due to better transmission of phonons.

\subsection{Mechanical tuning of RF cavity}

In a multicell cavity, each cell may have random fabrication errors which results in an offset of operating frequency of each cell. To optimize the net acceleration in a multicell cavity, it is necessary to achieve same operating frequency in each cell and hence uniform field profile. Cell to cell tuning is performed by applying plastic deformation of cell until the desired cell frequency is achieved. Manual tuning is very time consuming and requires lot of efforts and is not adequate for industrial production of cavities. Thus, semiautomated tuning machine is designed which can perform tuning very fast and can 
be operated even by non-RF experts.

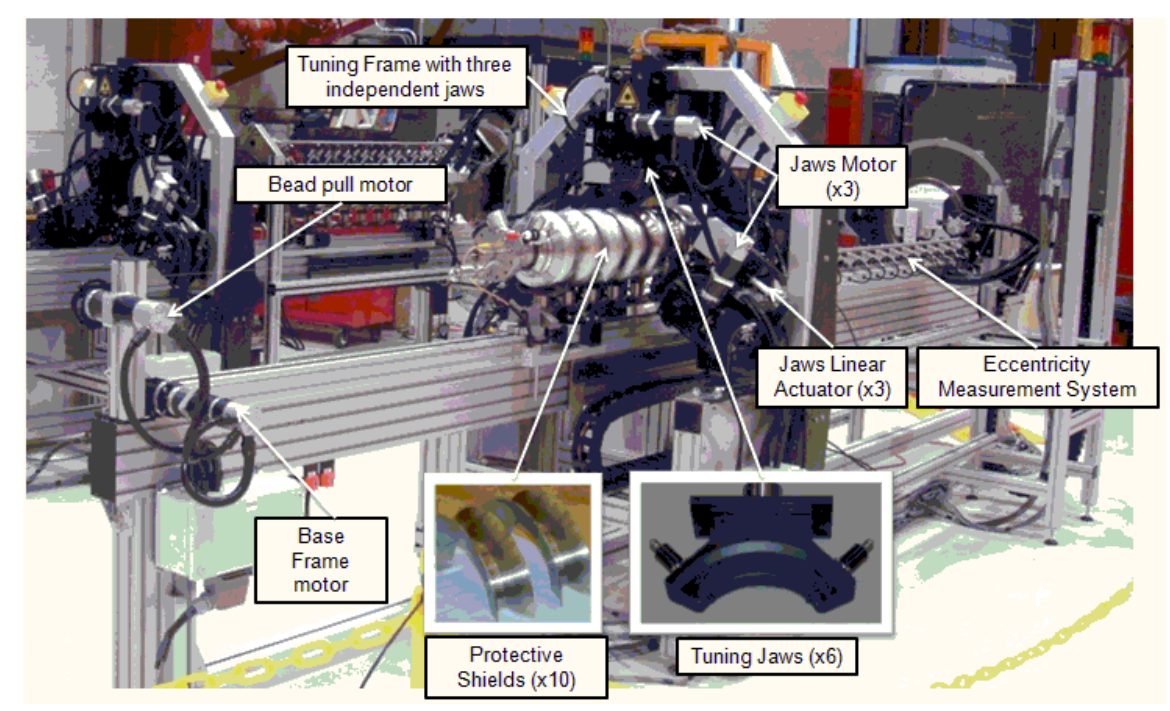

Figure 5.15: Tuning machine at Fermilab.
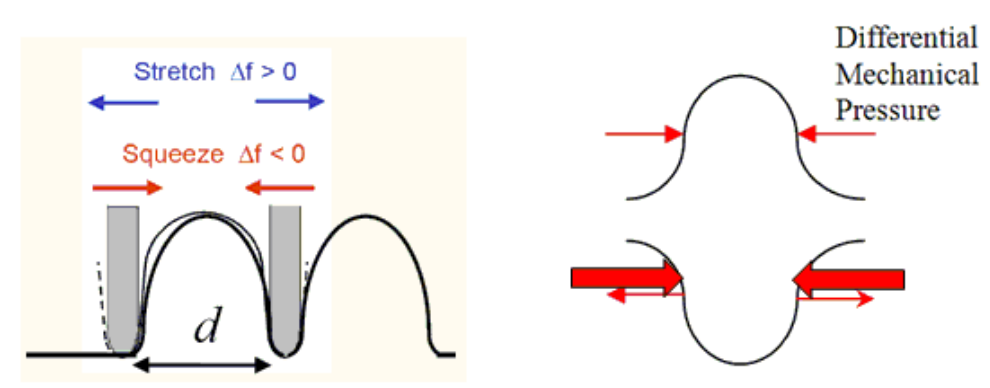

Figure 5.16: Tuning mechanism- Electrical model is used for frequency tuning of cavity (left) and mechanical model is used to tune cavity alignment(right).

Figure 5.15 shows tuning machine and its main components. Description of tuning machine and its components in detail is presented elsewhere [77]. Tuning of multicell cavity is based on electric model (Figure 5.16). According to this, stretching or squeezing of cell results in change of capacitance $\left(C \propto \frac{A}{d}, A\right.$ being area and $d$ is the cell length) which leads to change of resonance frequency of corresponding cell. Field flatness of cavity is measured using bead pull system [24]. To reduce the oscillation 
of metallic beads in perpendicular direction with respect to axis, the system is not string loop as in normal bead pull system but a string pulled from a reservoir. Bead movements in cavity causes disturbance in field which results in change in resonance frequency. Field amplitude $(|\vec{E}|)$ is proportional to square root of change in frequency, i.e., $|\vec{E}| \propto \sqrt{\Delta f}$.

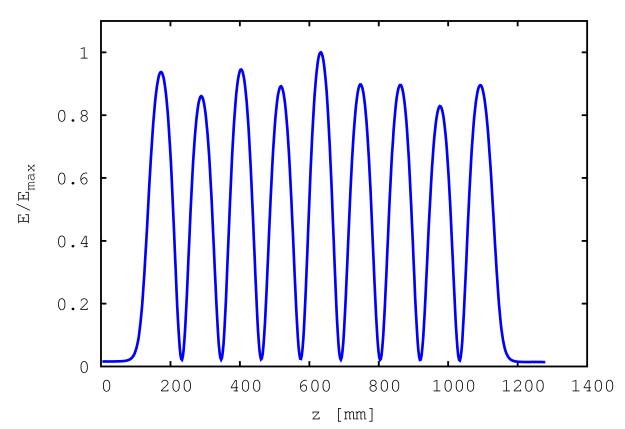

(a)

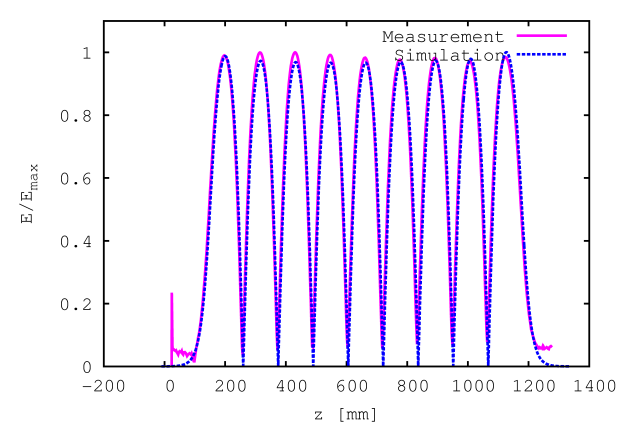

(b)

Figure 5.17: Distribution of field in $1.3 \mathrm{GHz}$, 9-cell cavity-(a) Field distribution without tuning and (b) field distribution of cavity after tuning in comparison with field distribution obtained numerically.

It can be seen from Figure 5.17a that fields are distributed randomly in each cell. Resonant frequency in each cell is tuned to operating frequency (1.3 GHz in this case). Field distribution of tuned cavity is shown in Figure 5.17b. Field flatness better than $98 \%$ is obtained after tuning. Measured field is compared with field calucluated numerically using SLANS to verify the reliability of numerical codes. It can be seen from Figure $5.17 \mathrm{~b}$ that fields are matched within $\pm 1 \%$ error.

For the entire tuning process, feedback of mechanical properties such as length and straightness of cavity and concentricity of the cells are useful to achieve a good tuning result. Tuning process includes not only frequency tuning but tuning of cell alignment also. It is necessary to obtain cell alignment within given limitation (0.2 $\mathrm{mm}$ for vertical alignment and 0.5 for eccentricity alignment for 9-cell, $1.3 \mathrm{GHz}$ cavity) which are decided by beam dynamics requirements. Tuning machine uses mechanical model to predict the bending angle at each cell. Bending of cell can be obtained by applying appropriate differential pressure on the outer walls of cell as shown in Figure 


\section{CAVITY FABRICATION AND CHARACTERIZATION}

5.16. A snapshot of cavity alignment before (left) and after (right) tuning of 9-cell 1.3 $\mathrm{GHz}$ cavity is shown in Figure 5.18 .
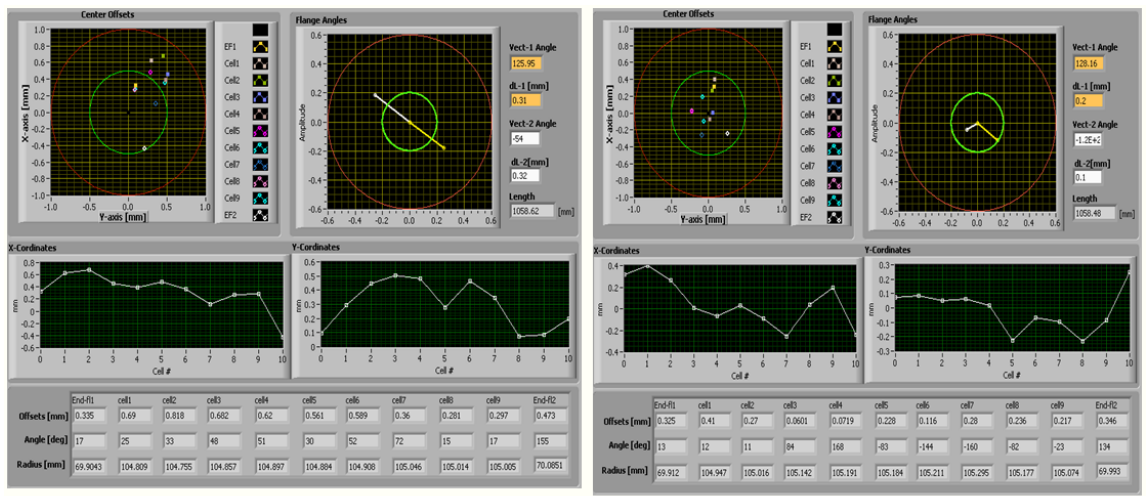

Figure 5.18: Cavity alignment before (left) and after (right) mechanical tuning of cavity.

\subsection{Experimental measurement of cavity: Vertical test}

Evaluation of cavity performance is performed prior to its installation in cryomodule. A bare cavity (no couplers and helium jacket) is tested at $2 \mathrm{~K}$ in $\mathrm{CW}$ RF regime. Quality factor $Q_{0}$ is measured as a function of the electric field in cavity. After cavity is equipped with input and pickup antennas and is vacuum sealed in clean room, it is placed in a cryostat, also called vertical test stand (VTS). A layout of test arrangement is shown in Figure 5.19 and discussed in [24, 78] with details. The input antenna is connected via a bellow to the cavity, The fundamental $\mathrm{TM}_{010}$ mode of the cavity is below the cut off frequency of the beam pipe. Thus, the electromagnetic field decays exponentially in the pipe. As a consequence the coupling strength of the input antenna, which is connected via a bellow to the cavity, can be varied by several orders of magnitude by adjusting the distance between antenna tip and cavity. The coupling strength can be optimized either by optimizing antenna length for fixed location of cavity in cryostat or adjusting cavity position relative to antenna tip for fixed length of antenna. Ideally, these tests are done at or near critical coupling and latter option of adjusting cavity position provides flexibility to achieve critical coupling for the temperature range from $4.2 \mathrm{~K}$ to 1.6 K. RF source requirements are only a few hundred watts at this mode of operation which is just enough power to overcome the wall and field emission losses. Figure 


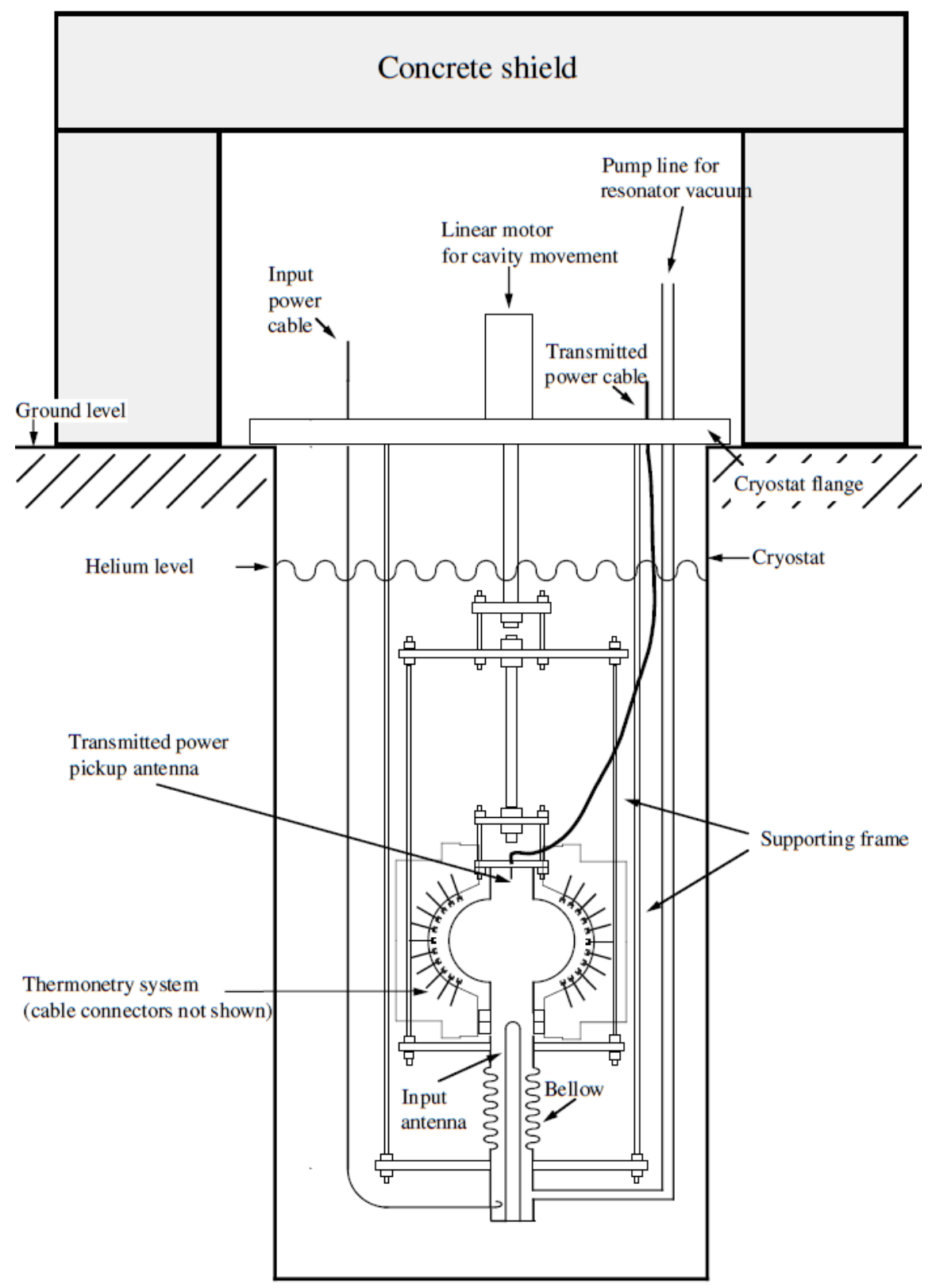

Figure 5.19: Schematic of the vertical cryostat used for cavity characterization at $2 \mathrm{~K}$ at Fermilab.

5.20 shows schematic of experimental setup for vertical test of cavity. A RF generator, which is locked exactly onto cavity resonant frequency, drives power amplifier which feed power to the cavity via cable and input antenna. The second antenna is used as a 


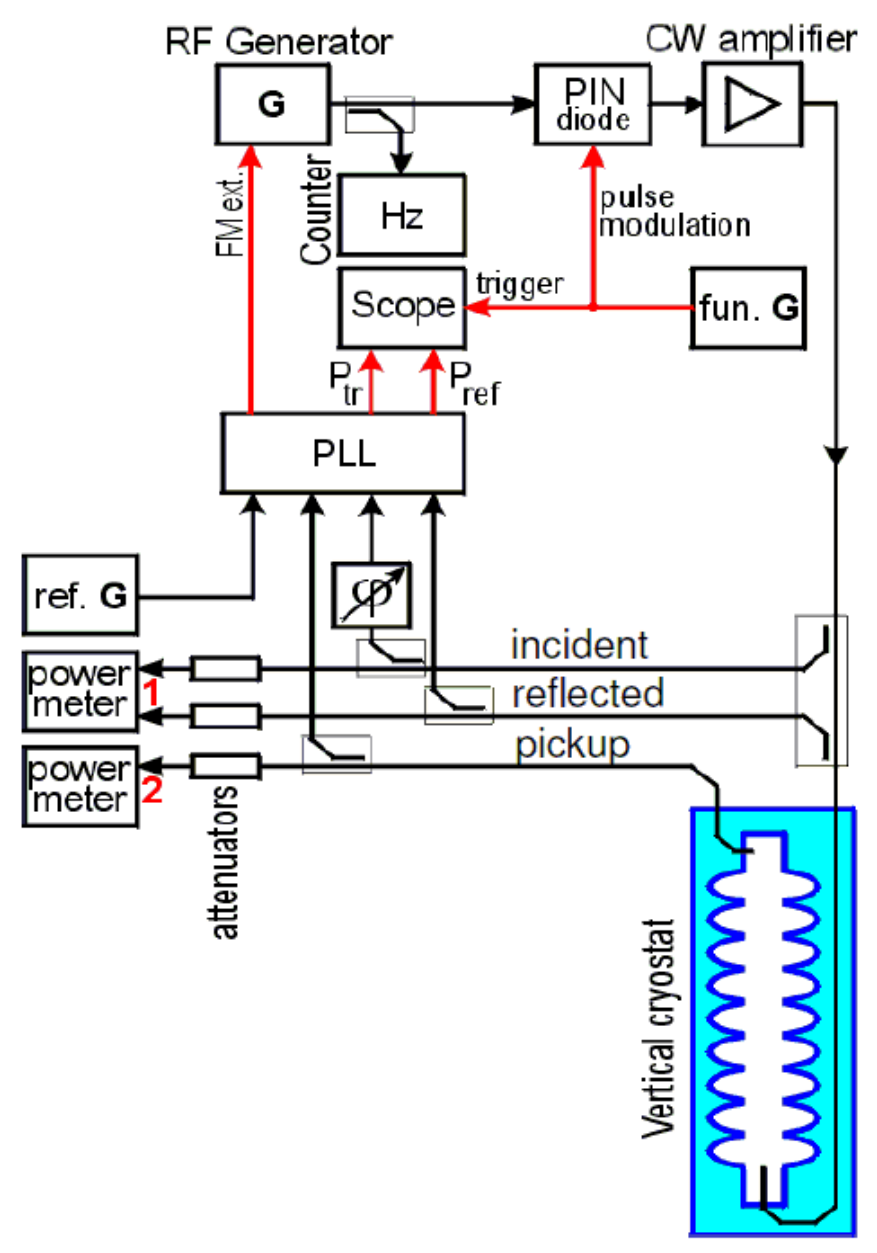

Figure 5.20: Schematic of experimental arrangement for vertical test showing required components which are needed for incident, reflected, pick-up power and feedback circuit.

pickup probe. It is adjusted with a much higher $\mathrm{Q}_{e x} \geq 10^{11}$ in order to avoid the signal transmission without resonance inside the cavity. It picks up only minimum power $\left(P_{t}\right)$ for monitoring the cavity field which is proportional to the square root of the power coupled out by the antenna. Pickup signals are also used for the feedback loop (Figure 5.20 .

As the SCRF cavities have a very high quality factor the width of the resonance curve is extremely small. Small changes in the helium pressure of the cryostat results in change of the resonance frequency of the cavity. Therefore the frequency generator has to be controlled with a phase-locked loop (PLL), where the phase difference between the input signal, taken from a directional coupler, and the pickup signal are compared. 
When the cavity is mounted in the cryostat, it will be first cooled with liquid helium at $4.2 \mathrm{~K}$ (atmospheric pressure). Cooling down to $2 \mathrm{~K}$ is achieved via pumping down the helium down to $\sim 30$ mbar. The cryogenic power available is about few hundred of Watts at $2 \mathrm{~K}$.

\subsubsection{Measurement of Quality factor}

All antennas are connected to calibrated power meters which allows to evaluate RF power losses in the cavity. The forward power $\left(\mathrm{P}_{f}\right)$, which is net power entering in cavity, is difference of incident power $\left(P_{i}\right)$ coming from source and reflected power $\left(P_{r}\right)$.

$$
P_{f}=P_{i}-P_{r}
$$

at steady state of cavity

$$
P_{i}=P_{r}+P_{t}+P_{d}
$$

where $P_{d}$ is the power dissipated to wall of the cavity. When incident power is switched off, the cavity enters into free decay state. Stored energy and hence electric field in the cavity decays exponentially. It can be expressed as:

$$
P_{\text {total }}=-\frac{d U}{d t}, \quad \text { with } \quad P_{\text {total }}=P_{d}+P_{t}+P_{e} ;
$$

where $P_{\text {total }}$ is total power stored in cavity and $P_{e}$ the power coupled out via the input antenna. Integrating differential equation $(5.6)$ one can obtain

$$
\begin{aligned}
U(t) & =U_{0} \cdot \exp \left(-\frac{t}{\tau_{L}}\right), \\
\text { where } \tau_{L} & =\frac{Q_{L}}{\omega_{0}} .
\end{aligned}
$$

The decay time constant $\tau_{L}$ is experimentally measured and is used to determine loaded quality factor $Q_{L}$. With measured values of $P_{e}, P_{t}$ and estimated values of $P_{d}$ and $Q_{L}$ one can determine $Q_{0}$. It can be written as:

$$
Q_{0}=Q_{L}\left(1+\beta_{t}+\beta_{e}\right) ;
$$

where coupling coefficient $(\beta)$ is $\beta_{k}=\frac{P_{k}}{P_{d}}=\frac{Q_{0}}{Q_{k}} ; \quad k=e, t$. Next step is to determine the relationship between $Q_{0}$ and $E_{a c c}$. The $P_{f}$ is increased to raise stored energy in cavity. At steady state of cavity $\left(P_{e}=0\right) Q_{0}$ is determined as:

$$
Q_{0}=\frac{Q_{t} P_{t}}{P_{d}} \text {. }
$$




\subsubsection{Measurement of Electric field level}

To understand the behavior of quality factor of cavity with accelerating gradient, corresponding accelerating field in cavity is measured as follows:

$$
E_{a c c}=\sqrt{\frac{P_{d} Q_{0}}{L^{2}} \cdot\left(\frac{R}{Q}\right)} ;
$$

where $L$ is length of cavity. $\frac{R}{Q}$ can be calculated from cavity design codes such as SUPERLANS, HFSS etc. Peak surface field $\left(E_{p k}\right)$ in cavity can be expressed as [24]:

$$
E_{p k}=\kappa_{e} \sqrt{U}=\kappa_{e} \sqrt{\frac{P_{d} Q_{0}}{\omega_{0}}}
$$

where $\kappa_{e}$ is proportionality factor of accelerating gradient and stored energy and depends on the geometrical layout of the cavity. It can be calculated using numerical codes.

\subsubsection{Measurement of single cell ILC cavity: TE1CAT003}

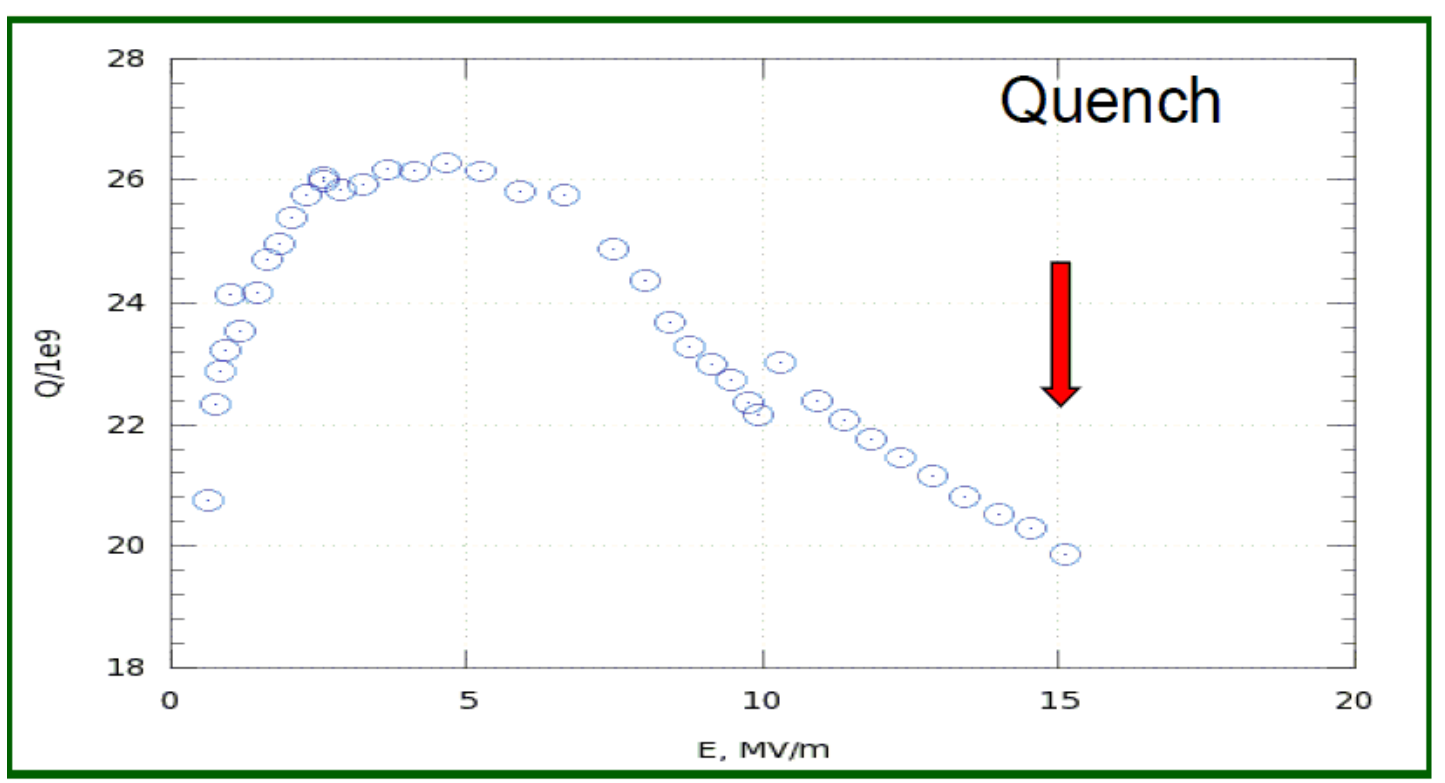

Figure 5.21: Performance of single cell ILC type TE1CAT003 cavity. 

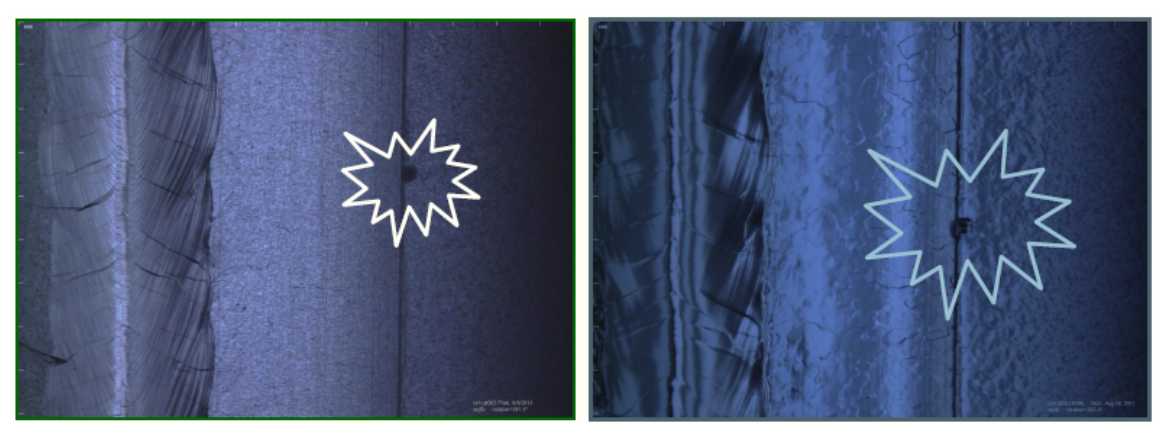

Figure 5.22: Defects in TE1CAT003 cavity- before (left) and after (right) chemical treatment of cavity.

Cavity TE1CAT003 is tested at vertical test facility (VTF) at Fermilab. Result is shown in Figure 5.21. Cavity is quenched at very low gradient of $15 \mathrm{MV} / \mathrm{m}$. To identify the reason of quench at this gradient, cavity is diagnosed.

The temperature mapping technique is adopted world wide to diagnose the cavity at cold temperature. The fast thermometry system [79, 80] is used to perform fast, accurate temperature measurements to observe quenches or other thermal abnormalities during a cavity test. It consists of temperature sensors which are placed at the outer wall of the cavity. The placement of sensors are flexible and can be changed depending on test requirements. The sensors are wired in series with a $1 \mathrm{~mA}$ current source and shunt resistor for measuring the current. For attaching the sensors to the cavity, Apiezon vacuum grease is used to provide good thermal contact, and a G10 band or thin nylon cord is used to hold the sensors in place. The $10 \mathrm{kHz}$ sampling rate is suitable for measuring both fast quenches and slower temperature rises.

TE1CAT003 cavity has been diagnosed and quench locations are identified using temperature distribution data obtained from fast thermometry. Cavity is later inspected with Kyoto camera. Figures 5.22 and 5.23 show appearance of pits after chemical treatment of surface. Before bulk EP of $120 \mu \mathrm{m}$, there were imprints of pits which are exposed after bulk chemical treatment of cavity. Pits cause enhancement of field in their neighborhood which results in more power dissipation. This localized increase in power dissipation ultimately leads to quench of cavity. Impacts of pits depend on their size. Pits and scratches near the equator region in cavity TE1CAT003 are main 


\section{CAVITY FABRICATION AND CHARACTERIZATION}
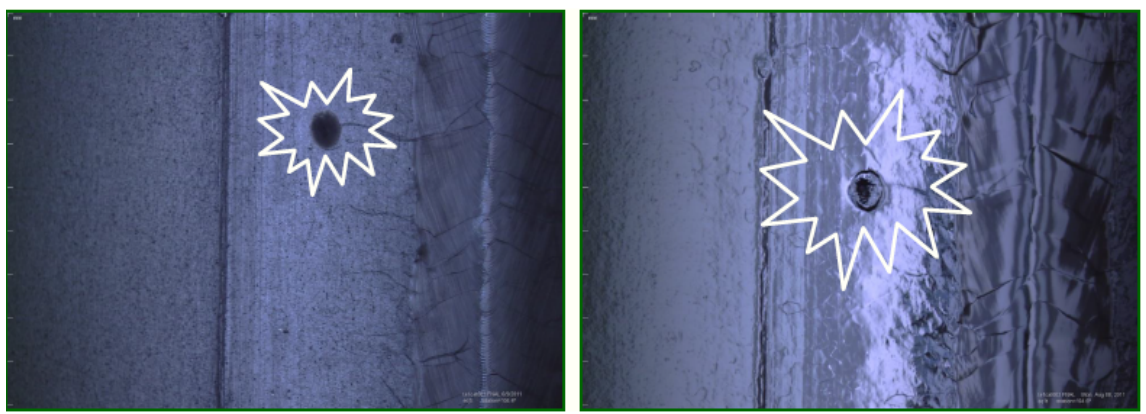

Figure 5.23: Another appearance of pit in TE1CAT003 cavity- before (left) and after (right) chemical treatment of cavity.

reasons that results in quench even at low gradient.

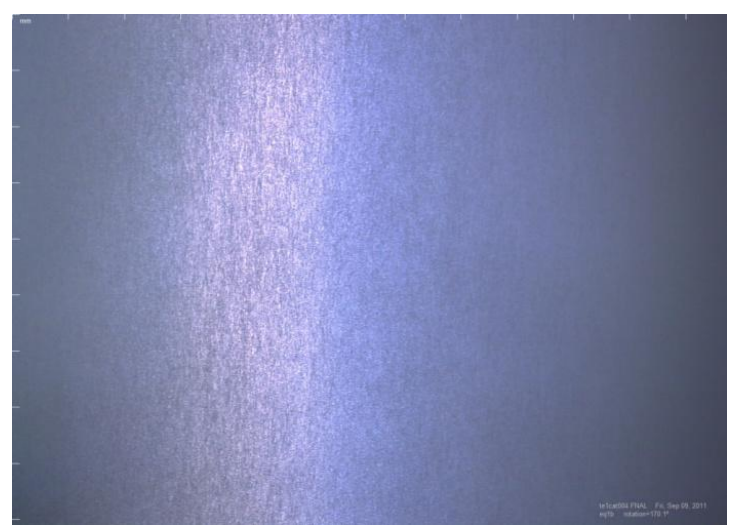

Figure 5.24: Cavity surface after tumbling and light EP treatment.

Cavity undergoes for further surface treatment. Tumbling is performed which is followed by light EP of $10 \mu \mathrm{m}$. The surface of cavity after this treatment are shown in Figure 5.24. It can be seen that pits have been disappeared and surface is smoother than earlier one. Cavity is baked at $120^{\circ}$ for 48 hours and then assembled again for cold vertical test. Cavity is tested at $2 \mathrm{~K}$ and $1.8 \mathrm{~K}$ and results are summarized in Figure 5.25. Cavity achieved gradient of $\sim 35 \mathrm{MV} / \mathrm{m}$ and $\sim 42 \mathrm{MV} / \mathrm{m}$ at $2 \mathrm{~K}$ and $1.8 \mathrm{~K}$ respectively. $Q_{0}$ obtained by cavity is also very high and it is $2 \times 10^{10}$ and $1.5 \times 10^{10}$ for $35 \mathrm{MV} / \mathrm{m}$ and $42 \mathrm{MV} / \mathrm{m}$ respectively. Global thermal instability happens at these gradients and cavity is quenched. Two soft multipacting regions were also found in between $15 \mathrm{MV} / \mathrm{m}$ 


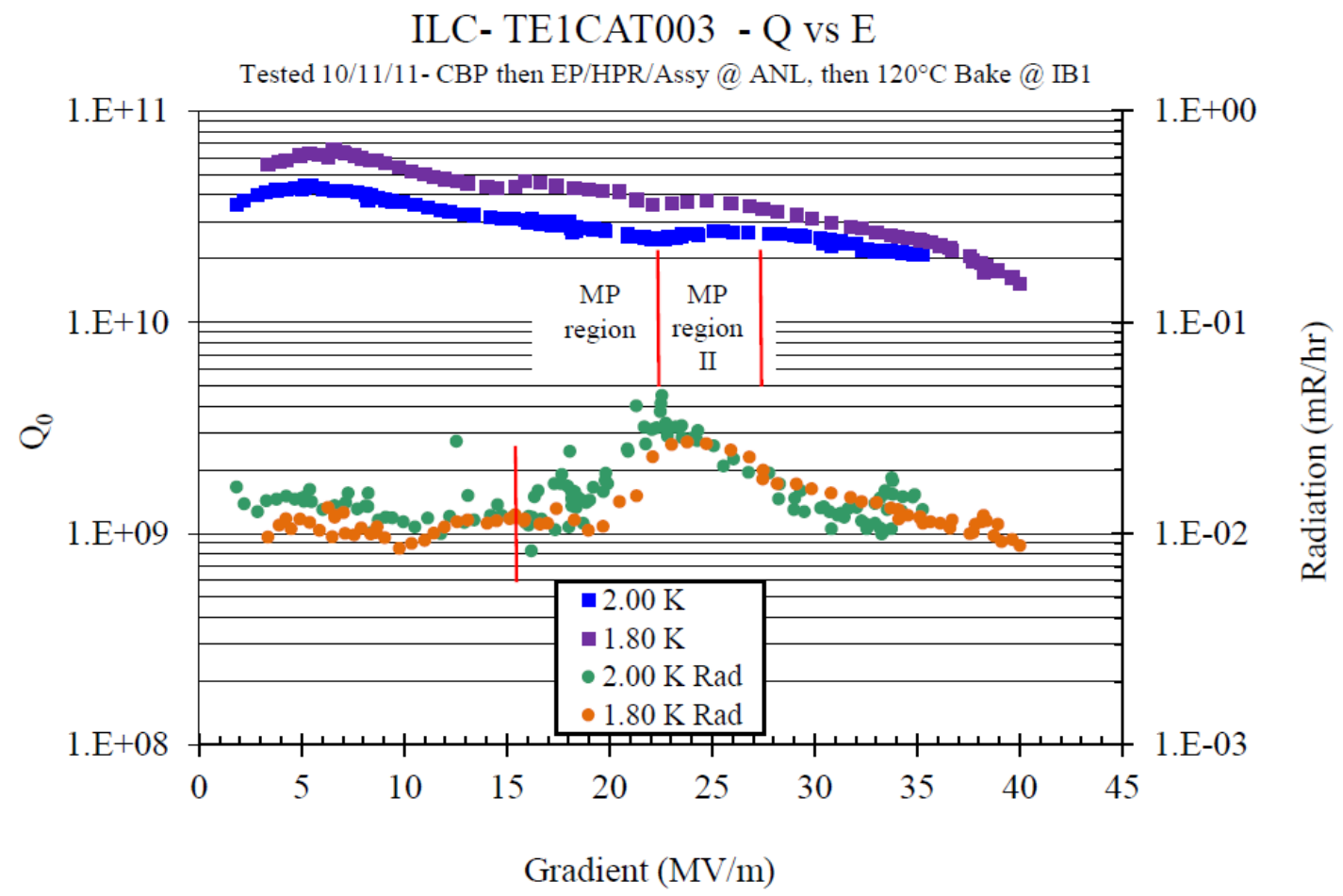

Figure 5.25: Performance of single cell ILC type TE1CAT003 cavity after surface treatment at $2 \mathrm{~K}$ and $1.8 \mathrm{~K}$.

to $27 \mathrm{MV} / \mathrm{m}$. There is no evidence of localized field emission. Systematic measurement of the X-ray intensity around SCRF cavities with high-sensitivity photo diodes shows no dramatic increase in X-ray which confirms absence of field emission. However, increase in X-rays in the range of accelerating gradient of $15 \mathrm{MV} / \mathrm{m}$ to $27 \mathrm{MV} / \mathrm{m}$ shows multipacting. It should be noted that hard multipacting and non-resonant electron loading due to field emission can result in increase of cavity surface temperature which can be seen from thermometry but no such indication is obtained in this case.

\subsection{Cavity installation in cryomodule}

After passing vertical test, cavity is accepted for final steps of installation. Cavity is fitted with input and higher order mode couplers, helium vessel, tuner and other auxiliary components. Cavity is rinsed using high pressure jet of ultra pure water for final cleaning of surface. High power testing is performed in horizontal test stand 
(HTS) to verify the performance of dressed cavity in accelerator. If cavity successfully passes this test, sealed cavity with helium vessel and cold part of coupler is ready for installation in cryomodule. 
6

\section{Summary}

Focus of accelerator community all over the world has been shifting towards the high intensity frontier. High intensity ion accelerators will be useful in numerous practical and basic science applications such as nuclear waste transmutation, spallation neutron source, material irradiation facilities, ADS, neutrino factory, rare decay processes etc. Fermilab has also proposed a high intensity proton facility, called Project-X, which supports several nuclear and high energy physics experiments simultaneously. Project$\mathrm{X}$ is based on $\mathrm{CW}$ linac which accelerates $\mathrm{H}^{-}$ion beam of average current $1 \mathrm{~mA}$ from kinetic energy of $2.5 \mathrm{MeV}$ to $3 \mathrm{GeV}$.

Recent advances in superconducting radio frequency (SCRF) technology have made it possible to construct high intensity proton accelerators with multi-milliamps of beam current and energies exceeding $1 \mathrm{GeV}$. Chapter 2 introduced RF description of cavity and a brief overview of superconductivity. Niobium is so far the first choice of superconducting material for the fabrication of superconducting cavities. Limiting mechanisms and simulation tools for cavity design are presented. This chapter also addressed the fundamentals of beam dynamics. Equations of motion of charge particle beam in transverse and longitudinal planes are discussed.

RF cavity is an important component of an accelerator. Thus, its design influences the operation of a linac. Cavity design is influenced by operational requirements and beam specifications such as operating frequency, number of cells etc. Cavity design also includes practical concerns which comprises of industrial production and use of available technology. Elliptical cavity is first choice for high energy acceleration due to its efficiency, compactness and ability to achieve higher accelerating gradient. Chapter 


\section{SUMMARY}

3 introduced topology of elliptical cavity geometry and the general procedure of shape optimization is discussed. Similar procedure is applied to design Project-X linac cavity. Preliminary proposal of Project-X facility was based on $8 \mathrm{GeV}$ pulsed linac. SqueezedILC $1.3 \mathrm{GHz}, \beta_{G}=0.81$ cavity was considered in high energy section of the linac to accelerate the beam from $\sim 0.4 \mathrm{GeV}$ to $1.2 \mathrm{GeV}$. This cavity was initially optimized for 11-cell. Length of 11-cell cavity is same as that of 9-cell, $1.3 \mathrm{GHz}, \beta_{G}=1 \mathrm{ILC}$ cavity. Thus, squeezed-ILC cavity can fit in a cryomodule designed for ILC cavity. 11-cell cavity is found to be sensitive toward trapping of HOMs. End cells are thus optimized to avoid potentially trapped HOMs and different end cells are used at both ends. Difficulty in handling of cavity with large number of cells and some practical constraints such as chemical residual and difficulties in surface processing can affect the industrial yield of the cavities. Earlier experiences of fabrication of 9-cell, $\beta_{G}=1$, $1.3 \mathrm{GHz}$ ILC cavity lead us to propose and design 9-cell, $\beta_{G}=0.81,1.3 \mathrm{GHz}$ cavity as an alternative of 11-cell cavity. Design procedure, RF parameters and geometrical parameters of optimized shape and HOM spectrum are presented in chapter 3 .

Supporting several experiments simultaneously in the Project-X facility requires high beam power. In order to increase the average beam current, operation mode of linac is changed from pulsed to $\mathrm{CW}$. The linac accelerates the beam from kinetic energy of $2.5 \mathrm{MeV}$ to $3 \mathrm{GeV}$. Two families of 5-cell, $650 \mathrm{MHz}$, elliptical cavities are designed. These cavities are optimized for $\beta_{G}=0.61$ and $\beta_{G}=0.90$. Intermediate energy section of the linac will use $\beta_{G}=0.61$ cavities to accelerate the beam from kinetic energy of $0.16 \mathrm{GeV}$ to $\sim 0.5 \mathrm{GeV}$. High energy section of the linac will use $\beta_{G}=0.90$ cavity for acceleration up to $3 \mathrm{GeV}$. RF and geometrical parameters for both of these cavities are presented. Cavities are investigated for potentially trapped HOMs. HOM spectrum and variation of concerned HOMs with beam velocity are also presented in chapter 3 .

When beam passes through a cavity, it induces HOMs which act back on beam and results in emittance dilution and power dissipation. Resonance excitations of HOMs result in degradation of beam quality and increase unnecessary cryogenic losses. In such scenario, damping of HOMs are required using HOM damper in a cavity. It is an expensive component and it may also degrade cavity performance. Analysis is performed to find the damping requirements in $\beta_{G}=0.61$ and $\beta_{G}=0.90$ cavities. Studies suggest that power dissipation due to HOM and emittance growth due to resonance excitation of HOMs are very low. It is possible to operate both families 
of cavities without HOM damper. Experimental studies show that HOM spectrum of cavities can be shifted by few hundred of $\mathrm{KHz}$ if fundamental mode is detuned and then re-tuned back to operating frequency. This method may be used to avoid resonance excitation of HOM in the absence of HOM damper.

High intensity of beam in Project-X can also result in some undesired effects which can degrade beam quality. Space charge effects and halo formation are some critical issues with high intensity ion linac. Layout of CW linac for Project-X facility is discussed in chapter 4. Assumptions and limitations which are used for designing the linac lattice are introduced. The linac uses five families of accelerating structures. Low energy part of linac consists of three families of SSR cavities which are used for the acceleration of beam from kinetic energy of $2.5 \mathrm{MeV}$ to $160 \mathrm{MeV}$. All SSR cavities operate at a frequency of $325 \mathrm{MHz}$. As mentioned earlier, intermediate and high energy sections of the linac will use two families of 5-cell elliptical $650 \mathrm{MHz}$ cavities. Solenoid magnets will be used for focusing of beam in low energy part and quadrupole dublets will be used in intermediate and high energy sections of the linac. A summary of beam line elements and energy transition are presented. Studies are performed to analyze beam dynamics for baseline lattice using simulation. Beam quality parameters such as emittance, r.m.s beam size and halo parameters are presented. Longitudinal and transverse acceptance of linac for baseline design are also studied. Transition from one section to another can result in shrinkage of acceptance. Thus, it is necessary to provide careful matching between sections to achieve large acceptance. Studies are performed to investigate most sensitive transition regions which limits acceptance of the linac. Operation of the linac in CW mode puts stringent tolerances on beam line elements, especially on elements in low energy part of linac. Failure of beam line elements may result in significant beam losses which can affect reliability of the linac. Thus, it is necessary to include this scenario during process of lattice design to have a robust lattice which may allow one to operate the linac even in the presence of failed elements. Local compensation scheme is demonstrated to compensate failure effects. These studies confirm that lattice is robust enough to allow compensation of at least one failed element.

Chapter 5 summarized the steps that cavity goes through before installation in accelerators. Cumulative experience of RF community results in preparing a procedure to fabricate SCRF cavities which can reproduce their performance. Although procedure is developed for $\beta_{G}=1,1.3 \mathrm{GHz}$, ILC cavity yet it can be adopted for other cavities 


\section{SUMMARY}

too. Fabrication of cavity starting with Niobium sheet is discussed along with various quality control tests. To achieve optimal performance of SCRF cavities, it is required to have smooth clean inner surface of a cavity. It can be achieved after chemical treatments of a cavity. Different methods of surface treatment and post surface purification are discussed. A bare cavity is tested on VTS before installation of other auxiliary components (e.g., power and HOM couplers). An example of vertical test of single cell ILC cavity is presented and its performance is reviewed. 


\section{References}

[1] S. D. Henderson. Project-X Functional Requirement specification. report RDR v 4.12, Fermilab, 2011. 1. 11

[2] S.D.Holmes et Al. Project-X Reference Design Report. report RDR v0.9., Fermilab. 1, 9

[3] H. Geiger and E. Mardden. On a Diffuse Reflection of the $\alpha$ Particles. Royal Society of London Proceedings Series A, 82:495-500, July 1909. 2

[4] R. J. Van de Graaff, K. T. Compton, And L. C. Van Atta. The Electrostatic Production of High Voltage for Nuclear Investigations. Phys. Rev., 43:149-157, Feb 1933. 2

[5] J.D.Cockcroft Et AL. Nature, 129:242, (1932). 2

[6] G. Ising. Ark. Mat. Fys. 18 (NO. 30), pages $1-4$, (1924). 3

[7] R. Wideroe. Arch. Electrorech. 21, page $387,(1928)$. 3

[8] L. Alvarez. Phys. Rev. 70, page 799, (1946). 3

[9] Ernest O. Lawrence and M. StanLey Livingston. The Production of
High Speed Light Ions Without the Use of High Voltages. Phys. Rev., 40:19-35, Apr 1932. 4

[10] M. LYNCh ET AL. SNS linac rf system overview. PAC, (1999). 5,9

[11] M. P. Kelly et Al. Cold test of a superconducting co-axial half wave cavity for RIA. LINAC, (2004). 5

[12] W.E. Fischer and Paul Scherrer. Application of spallation neutron sources. EPAC, (2002). 6

[13] A. Bettiny. Physics behind bound Standard Model. UFN, v. 171:977985, (2001). 6

[14] Y. Fukuda et AL. Constrains on Neutrino Oscillations Using 1258 Days of Super-Kamiokande Solar Neutrino Data. Phys. Rev. Lett., v. 86:5656-5660, (2001). 6

[15] A. Blanovsky. System for Nuclear Waste Transmutation Based on Target-Distributed Accelerators. IEEE, Nuclear Science Symposium, (1993). 8

[16] S. S. KAPOOR. Accelerator-driven sub-critical reactor system (ADS) for nuclear energy generation. Pramana- Journal of Physics, 59:941950, (2002). 9

[17] H. Langler. The European Spallation Source Study. NIM, B139, (1998). 9

[18] The Eurisol Collaboration. A feasibility study for a European Isotope Separation On-line Radioactive Ion Beam Facility. The eurisol report, 2003. 9 
[19] Y. YAmasaki. The JAERI/KEK Joint Project for High-Intensity Proton Accelerators. EPAC, (2002). 9

[20] S.D.Holmes. Project-X initial configuration document. report ICD v1.1, Fermilab. 11, 65, 80,82

[21] Q. JI. ion source, LEBT at LBNL: test goals, configuration and tasks. Project-X collaboration meeting, (2011). 15

[22] V. LabedeV eT AL. Broad band beam chopper for a $\mathbf{C W}$ proton linac at Fermilab. $P A C,(2011) .17$

[23] J. D. JaCKson. Classical Electrodynamics. Willey \& Sons, $3^{\text {rd }}$ edition, (1975). 24,25

[24] T. Hays H. Padamsee, J. Knobloch. RF Superconductivity for Accelerators. Willey-VCH, $2^{\text {nd }}$ edition, (2008). 38,39 44, 156, 157, 166, 168,172

[25] C. J. Gorter and H. B. G. Casimir. Two fluid model of superconductor. Z.Tech. Phys. 15,539, (1934). 38

[26] J. R. Schrieffer J. BArdeEn, J. COOPER. Theory of superconductivity. Physical review, 108:1175, (1957). 38

[27] A. B. PIPPARD. Impurities in superconductors. Proc. Roy. Soc., London, A203:98, (1953). 39

[28] F. PAlmer. Surface resistance of superconductor -Examples from $\mathrm{Nb}$ - 0 Systems. Proc. $3^{\text {rd }}$ SRF Conf., (1987). 40
[29] J. Matricon and D. Sainit-James. Superheating fields in superconductors. Phy. Lett. 24A:241, (1967). 40

[30] K. SAITo. Theoretical critical field in RF application. Proc. SRF Conf., (2003). 40

[31] T. Hays and H. Padamsee. Measuring the RF Critical field of $\mathrm{Pb}, \mathrm{Nb}$ and $\mathbf{N b}_{3}$ Sn. Proc. SRF Conf., (1997). 40

[32] C. Benvenuti et AL. superconducting cavities produced by magnetron sputtering of Niobium on copper. Proc. SRF Conf., (1987). 41

[33] B. Bonin. Material for Superconducting Cavities. CAS: Superconductivity in particle accelerator, pages .1912000, 1995. 42

[34] M. Peiniger et AL. Work on $\mathbf{N b}_{3} \mathbf{S n}$ Cavities at Wuppertal. Proc. SRF Conf., (1987). 42

[35] R. L. GENG. Limits in cavity performance. Tutorials SRF Conf., (2011). 42

[36] J. Halbritter P. Kneisel, R. VinCON. First results on elliptical shaped cavities. NIM, 188(3):669-70, 1981. 43

[37] R. Noer C. Reece H. Padamsee, W. Hartung and Q. S. Shu. RF field emission in superconducting cavities. Proc. SRF Conf., (1987). 44

[38] B. Bonin. Field emission in RF cavities. CAS: Superconductivity in particle accelerator. 44 
[39] R.H. Fowler AND L. Nordheim. First results on elliptical shaped cavities. Proc. Roy. Soc. London, A 119, 173. 44

[40] H. Safa M. Luong, B. Bonin And A. Legoff. High peak power processing up to $100 \mathrm{MV} / \mathrm{m}$ on various metallic samples. Proc. SRF Conf., (1995). 45

[41] K. W. Shepard H. Padamsee And R. Sundelin. Physics and accelerator applications of RF superconductivity. Ann. Rev. Nucl. Sci. 43, pages $635-86,(1993)$. 45

[42] D. G. Myakishev And V. P. Yakovlev. The New Possibilities of SuperLANS Code for Evaluation of Axisymmetric Cavities. $P A C$, (1995). 46

[43] Poisson/Superfish.

www.laacg.lanl.gov. 46

[44] ANSOFT.

HFSS.

www.ansoft.com/products/hf/hfss/.

46, 85

[45] CST Microwave Studio. www.cst.com/Content/Products/MWS. 46.

[46] Claes Johnson. Numerical simulation of partial differential equations by the finite element method. Cambridge, (1987). 47

[47] N. Pichoff. Introduction to RF Linear Accelerators. CAS, (2004). 53

[48] E. D. Courant and H. S. SNyder. Theory of alternating gradient synchrotron. Ann. Phys., 281:360-408, (1957). 55
[49] M. Conte and W. W. Mackay. An Introduction to the physics of particle accelerators. World scientific, 2 edition, (2008). 59

[50] F. SACHERER. RMS envelope equation with space charge. $4^{\text {th }} I E E E$ $P A C$. 60

[51] Frank Gerigk. Space charge and Beam halo in Proton linac. USCERN-JAPAN-RUSSIA Particle Accelerator School, Physics and Technology of Linear Accelerator Systems, 2002. 60

[52] C. K. Allen and T. P. Wangler. Beam halo definitions based upon moments of the particle distribution. Phys. Rev. ST Accel. Beams, 5, 124202, (2002). 61,132

[53] D. URIOT ET AL. http://irfu.cea.fr/Sacm/logiciels/index2.php. CEA. 62

[54] D. URIOT ET AL. http://irfu.cea.fr/Sacm/logiciels/index3.php. CEA. 62

[55] E. S. Lessner V. N. Aseev, P. N. Ostroumov and B. Mustapha. TRACK: The new beam physics code. $P A C,(2005) .63$

[56] J. P. Carneiro et AL. Multicell reduced beta elliptical cavities for a proton linac. $P A C$, pages 4899-901, (2009). 65, 82

[57] N. Solyak ET AL. Low beta structure for high energy part of Project X. PAC, pages 972-74, (2009). 65, 82

[58] T. P. Wangler. RF Linear Accelerator. Willey-VCH, $2^{\text {nd }}$ edition, Page 163 (2008). 73 
[59] M. Dohlus and V. Kaluuzhny. Relative non uniformity in the amplitude of the accelerating field along the M N cell TESLA super cavities , Report No. report 1998-26, DESY. 73

[60] D. Proch. The TESLA cavity: Design consideration and RF properties. SRF Conf., pages 382-97, (1993). 81

[61] B. Aune ET AL. Superconducting TESLA cavities Phys. Rev. ST Accel. Beams, 3(9). 81

[62] N. Toge N. Phinney And N. WALKER. ILC reference design report - Accelerator. 81, 90

[63] R. Doremus M. W. McGee And C. R. WAnds. Mechanical Stability of Type IV cryomodule. $P A C$, pages 2277-79, (2007). 81

[64] V. Yakovlev et AL. HOMs in High Energy part of the Project $\mathrm{X}$ linac. SPL HOM Workshop, (2009). 89

[65] A. Vostrikov A. SAini Et AL. Higher Order Modes in $650 \mathrm{MHz}$ sections of the Project-X Linac. TD-Notes, http://tdserver1.fnal.gov/tdlibry/TDNotes/2011\%20Tech\%20Notes/TD11-008.pdf, (2011). 114, 118

[66] N. Solyak ET AL. Longitudinal and transverse effects of HOMs in the Project X linac. IPAC, (2011). 117

[67] J. Stovall et AL. Expected beam performance of SNS linac. $P A C$, (2001). 124
[68] P. N. Ostroumov. Physics design of the $8 \mathrm{GeV} \mathrm{H}^{-}$linac. New Journal of Physics, 8 281. 124

[69] R. S. Mills ET AL. Design self matching linac. Linear accelerator conference, (1984). 124

[70] I. Gonin ET AL. Single spoke cavities for low energy part of CW linac of Project-X. IPAC, (2010). 126, 128

[71] L. Ristori et AL. Design of Single spoke resonators for Project-X. PAC, (2011). 127

[72] N. Solyak et al. Design of Project X CW linac. (2010). 134

[73] ANSYS. www.ansys.com. 155

[74] P. Knesiel And B. Lewis. Advanced surface cleaning methidsThree years of experience with high pressure ultrapure water rinising of superconducting cavities. Particle acclerator, 53:97-121, (1996). 160

[75] P. KNEISEl. Surface preparation of Niobium. Proc. SRF Conf., (1980). 162

[76] K. Saito. Superiority of Electropolishing over Chemical Polishing on High Gradients. Proc. SRF Conf., (1997). 163

[77] D. Reschke. Preparatory procedure and equipment for the European x-ray free electron laser cavity implementation. Phys. Rev. ST Accel. Beams, 13, 071001, (2010). 166

[78] J. P. Ozelis ET AL. Design and Commissioning of Fermilab's Vertical Test Stand for ILC SRF Cavities. $P A C$, (2007). 168 
[79] D. OrRis ET AL. Fast Thermometry for Superconducting RF Cavity Testing. PAC, (2007). 173
[80] C. M. Ginsburg ET Al. Diagnostic Instrumentation for the Fermilab Vertical Cavity Test Facility. SRF, (2007). 173 